\title{
Catalytic Asymmetric (3 + 3) Cycloaddition of Oxyallyl Zwitterions with $\alpha$-Diazomethylphosphonates
}

Yan Liu, Xian Peng, Rui She, Xin Zhou,* and Yungui Peng*

Key Laboratory of Applied Chemistry of Chongqing Municipality, School of Chemistry and Chemical Engineering, Southwest University, Chongqing 400715, China

*E-mail address: xinzhou@ @wu.edu.cn; pengyungui@hotmail.com; pyg@ swu.edu.cn

\section{Contents}

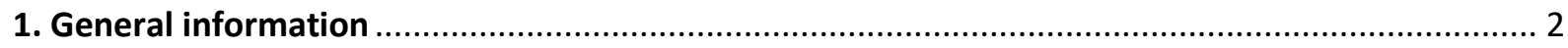

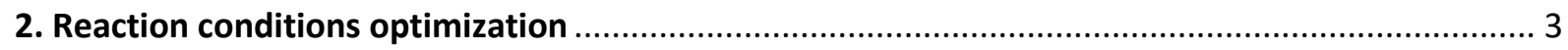

3. General procedure for the catalytic asymmetric $(3+3)$ cycloaddition reaction ....................... 4

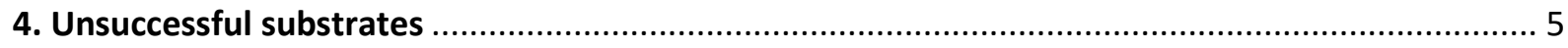

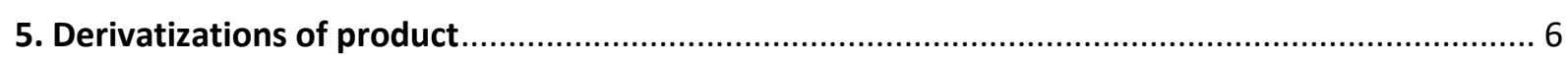

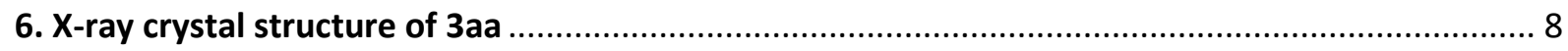

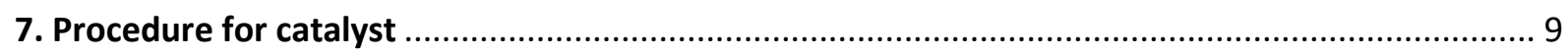

8. General procedure for preparation of $\boldsymbol{\alpha}$-haloketones .................................................... 10

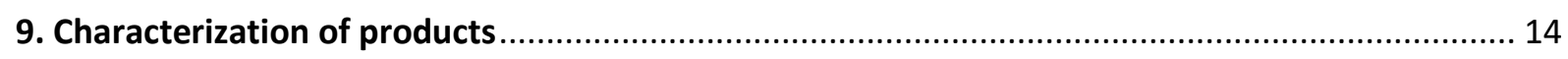

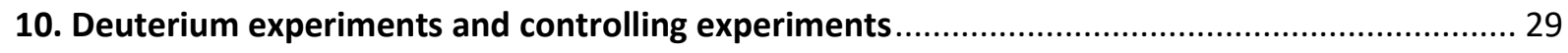

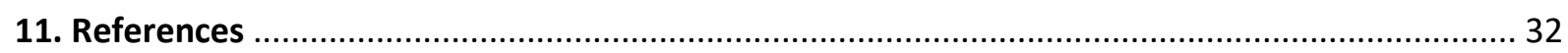

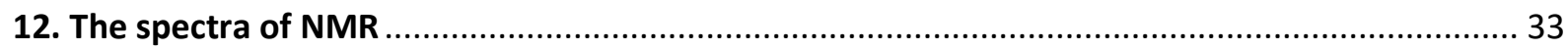

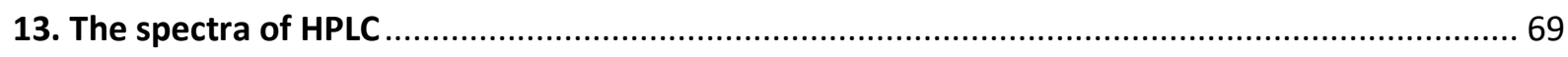




\section{General information}

Unless stated otherwise, all reactions were carried out under argon atmosphere. All the reactions that need to be heated, the oil bath is used as a heating source. All solvents were purified by standard procedures and distilled prior to use. Reagents obtained from commercial source were used without further purification. Petroleum ether and ethyl acetate for flash column chromatography was distilled before use. All reactions were monitored by TLC with silica gel coated plates. Flash column chromatography was performed on silica gel (300-400 mesh). NMR spectra were recorded on Bruker Avance $600 \mathrm{MHz}$ instruments. Chemical shifts $(\delta)$ are given in ppm relative to TMS, coupling constants $(J)$ in Hz. Data are reported as follows: chemical shift in ppm $(\delta)$, multiplicity $(\mathrm{s}=$ singlet, $\mathrm{d}=$ doublet, $\mathrm{t}=$ triplet, $\mathrm{q}=$ quartet, brs $=$ broad singlet, $\mathrm{m}=$ multiplet $)$, coupling constant $(\mathrm{Hz})$, and integration. ${ }^{1} \mathrm{H}$ NMR chemical shifts are reported in ppm relative to tetramethylsilane (TMS) with the solvent signal as the internal standard $\left(\mathrm{CDCl}_{3}\right.$ at $\left.7.26 \mathrm{ppm}\right) .{ }^{13} \mathrm{C} \mathrm{NMR}$ chemical shifts are reported in ppm from tetramethylsilane (TMS) with the solvent resonance as the internal standard $\left(\mathrm{CDCl}_{3}\right.$ at $77.16 \mathrm{ppm})$. Melting points were determined on an X-6 digital melting-point apparatus and were uncorrected. Optical rotations were measured on a Perkin Elmer 341 Polarimeter at $\lambda=$ $589 \mathrm{~nm}$. IR spectra were recorded using a Perkin Elmer Spectrum 1 machine. Absorption maxima (vmax) are reported in wavenumbers $\left(\mathrm{cm}^{-1}\right)$. Analytical high performance liquid chromatography (HPLC) was carried out on WATERS 510 instrument (2487 Dual $\lambda$ Absorbance Detector and 515 HPLC Pump) using chiral column (Chiralpak IC, IF columns were purchased from Daicel Chemical Industries). ESI HRMS (Bio TOF Q) spectra were recorded on P-SIMS-Gly of Bruker Daltonics Inc. 


\section{Reaction conditions optimization}

Table S1: The effect of base, solvent and temperature on the reaction ${ }^{a}$

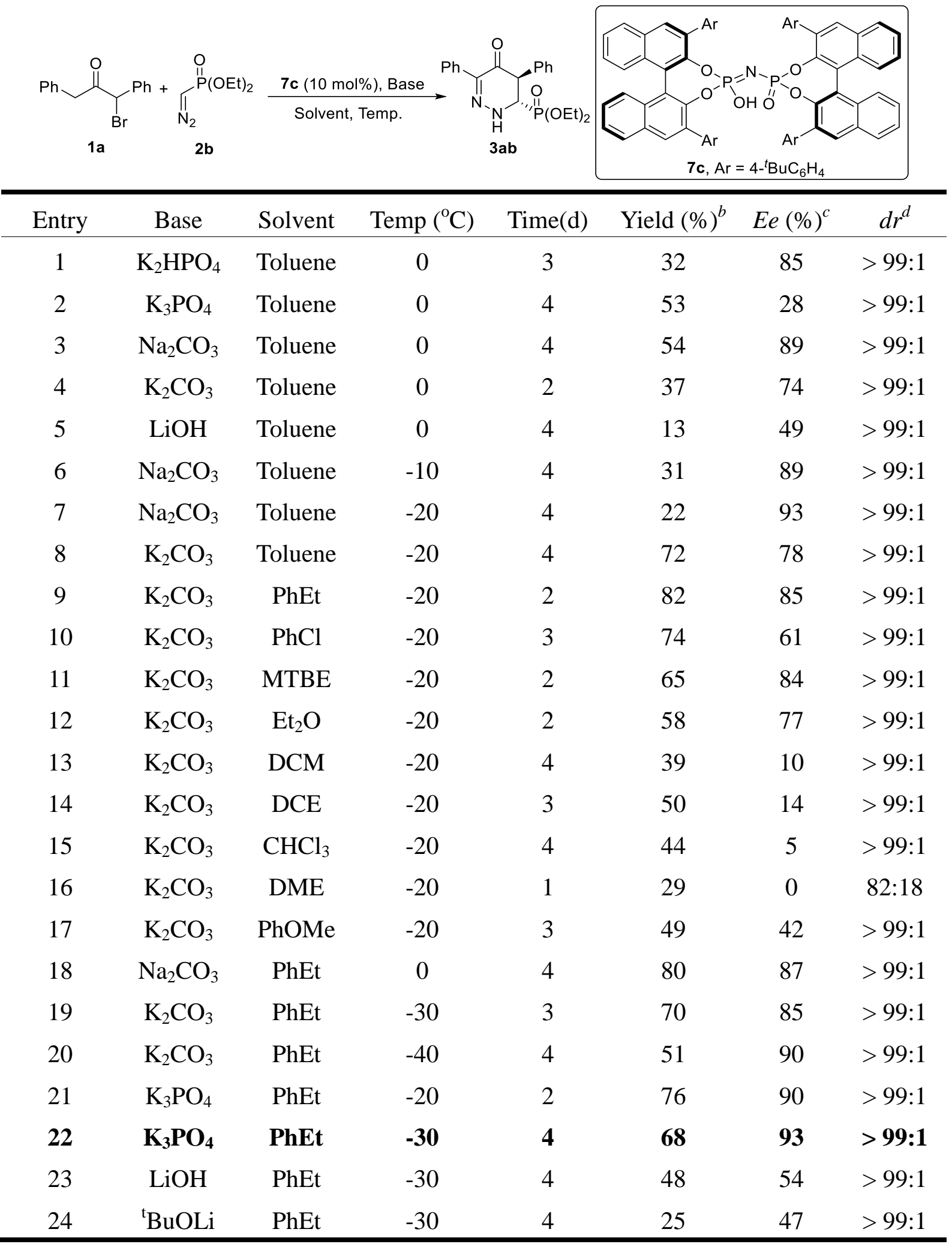

${ }^{a}$ Unless otherwise specified, the reaction was carried out with $\mathbf{1 a}(0.1 \mathrm{mmol}), \mathbf{2 b}(0.1 \mathrm{mmol})$, base $(0.2$ mmol), $7 \mathbf{c}(10 \mathrm{~mol} \%)$ in $0.5 \mathrm{~mL}$ of solvent. ${ }^{b}$ Isolated yield. ${ }^{c}$ Determined by chiral HPLC. ${ }^{d}$ Determined by ${ }^{1} \mathrm{H}$ NMR. 
Table S2: The additional optimization of reaction conditions ${ }^{a}$

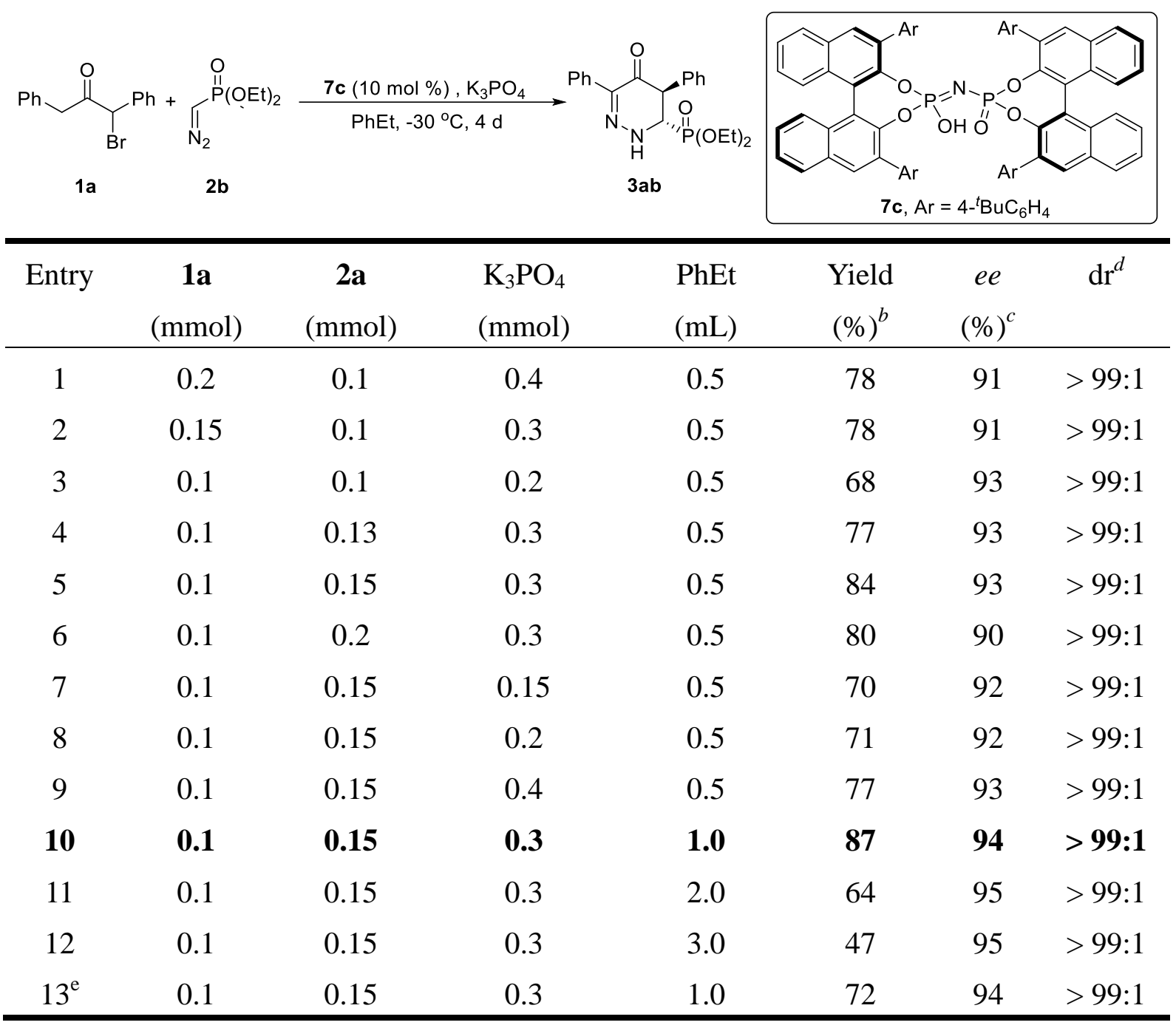

${ }^{a}$ Unless otherwise specified, the reaction was carried out with $\mathbf{1 a}, \mathbf{2 b}, \mathrm{K}_{3} \mathrm{PO}_{4}$ and $\mathbf{7 c}(10 \mathrm{~mol} \%)$ in the $\mathrm{PhEt}$ at $-30{ }^{\circ} \mathrm{C}$ for 4 days. ${ }^{b}$ Isolated yield. ${ }^{c}$ Determined by chiral HPLC. ${ }^{d}$ Determined by ${ }^{1} \mathrm{H}$ NMR. ${ }^{\mathrm{e}} \mathrm{Catalyst}$ loading was $5 \mathrm{~mol} \%$.

\section{General procedure for the catalytic asymmetric $(3+3)$ cycloaddition reaction}

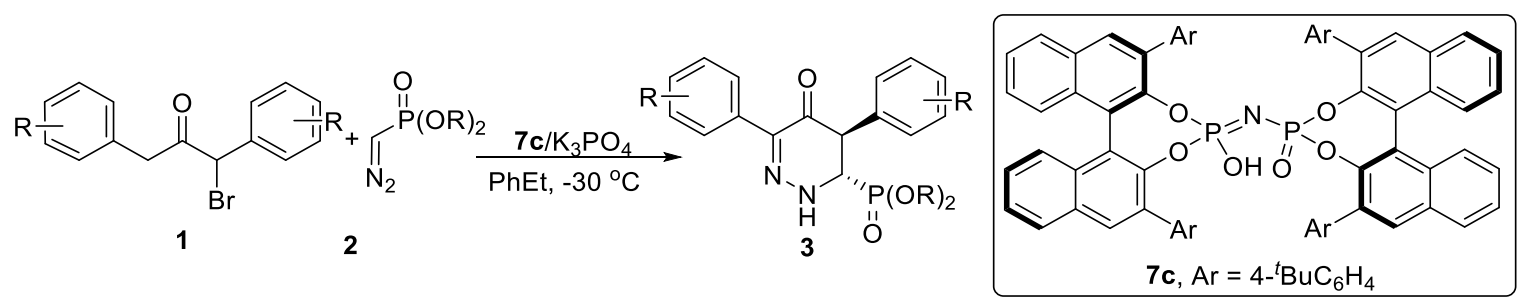

Unless specified otherwise, (diazomethyl)phosphonate $2(0.15 \mathrm{mmol})$ was added to a mixture of chiral catalyst $7 \mathrm{c}(0.01 \mathrm{mmol}, 10 \mathrm{~mol} \%), \mathrm{K}_{3} \mathrm{PO}_{4}(0.3 \mathrm{mmol})$ and $\alpha$-haloketone 1 $(0.1 \mathrm{mmol})$ in anhydrous ethylbenzene $(1.0 \mathrm{~mL})$ at $-30{ }^{\circ} \mathrm{C}$. The mixture was stirred vigorously 
for 4-5 days. The reaction was then treated with acetic acid $(30 \mu \mathrm{L})$. The diastereomeric ratio was determined by analyzing the ${ }^{1} \mathrm{H}$ NMR spectrum of the crude reaction mixture. Purification was performed by column chromatography or preparative thin layer chromatography on silica gel using petroleum ether/acetone as the eluents.

\section{1 mmol-scale experiment}

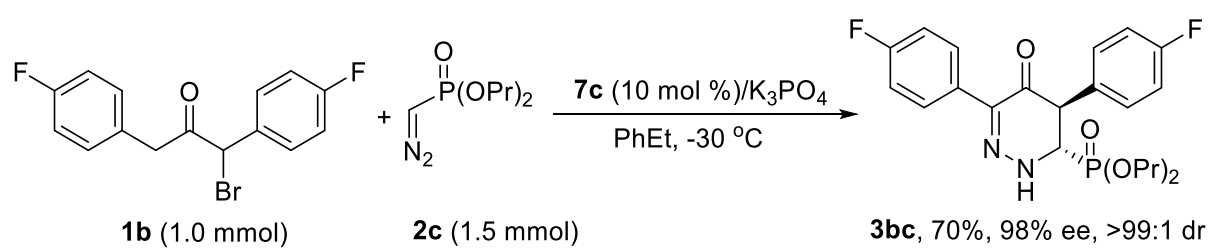

Dipropyl (diazomethyl)phosphonate 2c (0.309 g, $1.5 \mathrm{mmol}, 1.5$ equiv.) was added to a mixture of chiral catalyst $7 \mathrm{c}\left(121.0 \mathrm{mg}, 0.1 \mathrm{mmol}, 0.1\right.$ equiv.), $\mathrm{K}_{3} \mathrm{PO}_{4}(0.637 \mathrm{~g}, 3.0 \mathrm{mmol} 3.0$ equiv.) and $\alpha$-haloketone $\mathbf{1 b}(0.329 \mathrm{~g}, 1.0 \mathrm{mmol} 1.0$ equiv. $)$ in anhydrous ethylbenzene (8.0 $\mathrm{mL}$ ) at $-30{ }^{\circ} \mathrm{C}$. The mixture was stirred vigorously for 4 days. The reaction was then treated with acetic acid $(200 \mu \mathrm{L})$. The diastereomeric ratio was determined by analyzing the ${ }^{1} \mathrm{H}$ NMR spectrum of the crude reaction mixture. Purification was performed by column chromatography on silica gel using petroleum ether/acetone (8:1 to $3: 1)$ as the eluents, and obtained the corresponding product $3 \mathbf{b c}(0.315 \mathrm{~g}, 70 \%$ yield, $98 \%$ ee, $>99: 1 d r)$.

\section{Unsuccessful substrates}

For $\alpha$-substituted (Br, OTs) pentan-3-one, cyclopentanone and cyclohexanone, no desired $(3+3)$ cycloaddition products were obtained under the standard conditions. However, when one of the aryl substituents was replaced by methyl, the desirable $(3+3)$ cycloaddition product was obtained smoothly although with only a little enantioselectivity (3wc, $25.2 \mathrm{mg}$, $68 \%$ yield, $33 \%$ ee, $>99: 1 d r, 6.0 / 1 r r)$.

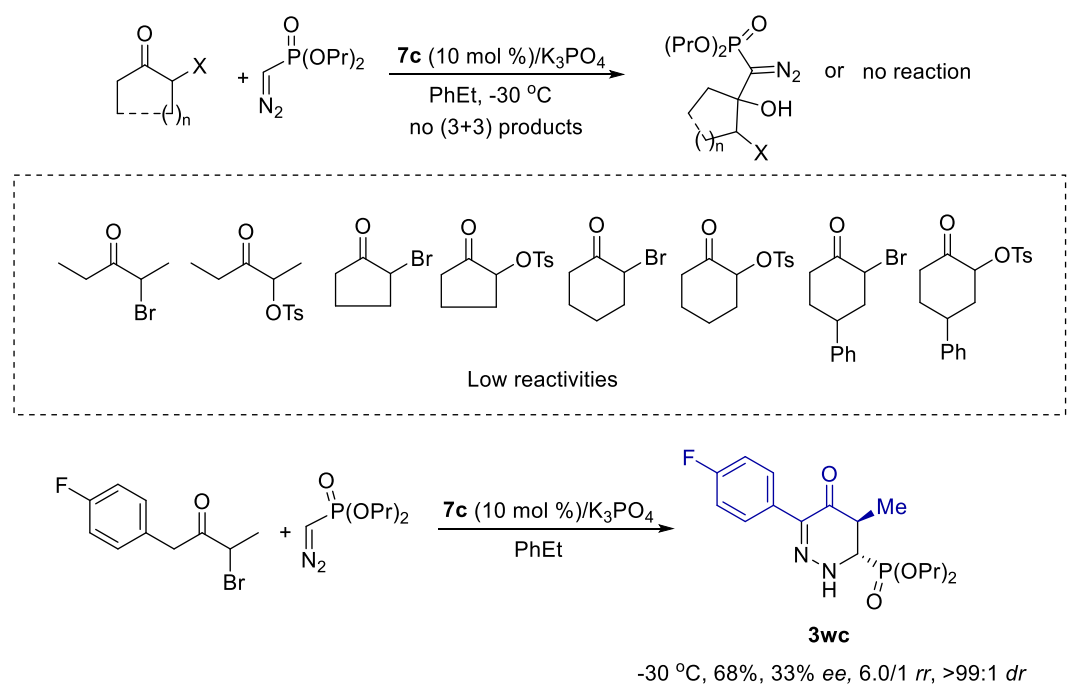




\section{Derivatizations of product}

(a)

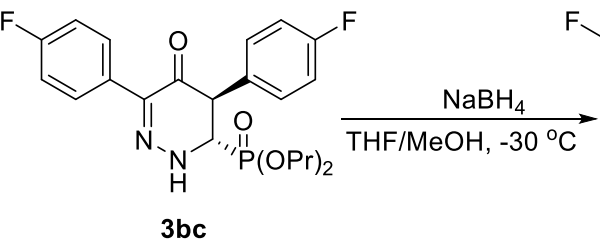

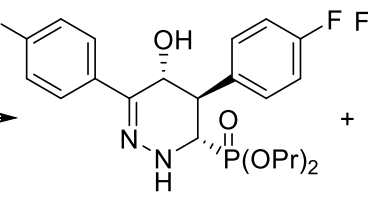

$10,50 \%, 98 \%$ ee

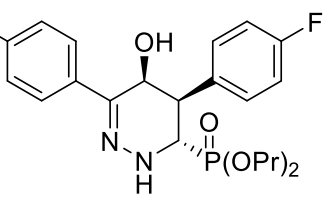

$10^{\prime}, 50 \%, 94 \%$ ee

(b)
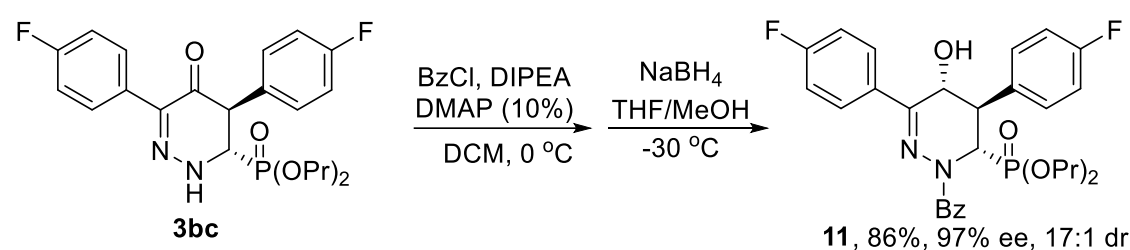

\subsection{Procedure for the preparation of 5-hydroxy-pyridazin derivatives 10/10'}

$\mathrm{NaBH}_{4}(25.0 \mathrm{mg}, 0.65 \mathrm{mmol})$ was added to the solution of the product $3 \mathbf{b c}(59.0 \mathrm{mg}, 0.13$ $\mathrm{mmol})$ in $\mathrm{THF} / \mathrm{MeOH}(1.5 / 0.5 \mathrm{~mL})$ at $-30{ }^{\circ} \mathrm{C}$. The reaction mixture was stirred until the starting material was disappeared after $3 \mathrm{~h}$. The reaction solution was treated with acetic acid $(100 \mathrm{uL})$ and purified by column chromatography on silica gel to give $\mathbf{1 0}(29.4 \mathrm{mg}, 50 \%$ yield as a colorless oil) and $\mathbf{1 0}^{\prime}$ (29.2 $\mathrm{mg}, 50 \%$ yield as a white solid).

Dipropyl ((3S,4R,5R)-4,6-bis(4-fluorophenyl)-5-hydroxy-2,3,4,5-tetrahydropyridazin-3yl)phosphonate (10).

Colorless oil; $[\alpha]_{D}^{20}=-83.9^{\circ}(c=1.0$, DCM $)$; HPLC (IC, n-hexane $/{ }^{i} \operatorname{PrOH}=80 / 20$, flow rate $=$ $0.5 \mathrm{~mL} / \mathrm{min}, \mathrm{I}=254 \mathrm{~nm}$ ): $t_{\mathrm{R}}=43.2 \mathrm{~min}$ (major), $35.2 \mathrm{~min}(\operatorname{minor}) ;{ }^{1} \mathrm{H} \mathrm{NMR}(600 \mathrm{MHz}$, Acetone- $\left.d_{6}\right) \delta{ }^{1} \mathrm{H}$ NMR $\left(600 \mathrm{MHz}\right.$, Acetone- $\left.d_{6}\right) \delta$ 7.94-7.77 (m, 2H), $7.50(\mathrm{~s}, 1 \mathrm{H}), 7.30-7.21$ (m, 2H), 7.12-7.02 (m, 4H), $5.77(\mathrm{~d}, J=11.2 \mathrm{~Hz}, 1 \mathrm{H}), 4.30(\mathrm{~d}, J=9.7 \mathrm{~Hz}, 1 \mathrm{H}), 4.14-4.04(\mathrm{~m}$, 4H), $3.82(\mathrm{dd}, J=12.9,3.3 \mathrm{~Hz}, 1 \mathrm{H}), 3.70-3.63(\mathrm{~m}, 1 \mathrm{H}), 1.81-1.72(\mathrm{~m}, 2 \mathrm{H}), 1.72-1.64(\mathrm{~m}$, $2 \mathrm{H}), 1.00(\mathrm{t}, J=7.4 \mathrm{~Hz}, 3 \mathrm{H}), 0.94(\mathrm{t}, J=7.4 \mathrm{~Hz}, 3 \mathrm{H}) ;{ }^{13} \mathrm{C}$ NMR $\left(150 \mathrm{MHz}\right.$, Acetone- $\left.d_{6}\right) \delta$ $162.2(\mathrm{~d}, J=244.4 \mathrm{~Hz}), 161.8(\mathrm{~d}, J=243.6 \mathrm{~Hz}), 139.9,137.8$ (dd, $J=16.4,3.2 \mathrm{~Hz}), 134.4(\mathrm{~d}$, $J=2.4 \mathrm{~Hz}), 129.4(\mathrm{~d}, J=7.9 \mathrm{~Hz}), 127.1(\mathrm{~d}, J=7.8 \mathrm{~Hz}), 115.1(\mathrm{~d}, J=21.4 \mathrm{~Hz}), 114.4(\mathrm{~d}, J=$ $21.5 \mathrm{~Hz}), 68.7$ (d, $J=7.4 \mathrm{~Hz}), 68.0(\mathrm{~d}, J=7.6 \mathrm{~Hz}), 62.6,51.4(\mathrm{~d}, J=150.0 \mathrm{~Hz}), 44.3(\mathrm{~d}, J=$ $4.3 \mathrm{~Hz}), 23.7(\mathrm{~d}, J=5.6 \mathrm{~Hz}), 23.7(\mathrm{~d}, J=5.4 \mathrm{~Hz}), 9.5,9.3 ;{ }^{19} \mathrm{~F} \mathrm{NMR}\left(565 \mathrm{MHz}, \mathrm{CDCl}_{3}\right) \delta$ -114.04, -114.83; ${ }^{31} \mathrm{P}$ NMR (243 MHz, Acetone- $d_{6}$ ) $\delta$ 23.95; HRMS (ESI): $\mathrm{m} / \mathrm{z}$ calcd. for $\mathrm{C}_{22} \mathrm{H}_{27} \mathrm{~F}_{2} \mathrm{~N}_{2} \mathrm{NaO}_{4} \mathrm{P}[\mathrm{M}+\mathrm{Na}]^{+}: 475.1569$, found: 475.1568 .

Dipropyl ((3S,4R,5R)-4,6-bis(4-fluorophenyl)-5-hydroxy-2,3,4,5-tetrahydropyridazin-3yl)phosphonate $\left(10^{\prime}\right)$.

White solid; mp $341.2-342.5^{\circ} \mathrm{C}$; $[\alpha]_{\mathrm{D}}^{20}=-39.3^{\circ}(c=1.0$, DCM); HPLC (IC, n-hexane $/ \mathrm{PrOH}$ $=80 / 20$, flow rate $=0.5 \mathrm{~mL} / \mathrm{min}, \mathrm{I}=254 \mathrm{~nm}): t_{\mathrm{R}}=41.0 \min ($ major $), 43.8 \min ($ minor $) ;{ }^{1} \mathrm{H}$ 
NMR (600 MHz, Acetone- $\left.d_{6}\right) \delta 7.82-7.76(\mathrm{~m}, 2 \mathrm{H}), 7.51-7.45(\mathrm{~m}, 2 \mathrm{H}), 7.11-7.02(\mathrm{~m}, 4 \mathrm{H})$, $6.81(\mathrm{br}, 1 \mathrm{H}), 4.65(\mathrm{br}, 1 \mathrm{H}), 4.50(\mathrm{t}, J=4.0 \mathrm{~Hz}, 1 \mathrm{H}), 4.15(\mathrm{dd}, J=12.4,6.5 \mathrm{~Hz}, 1 \mathrm{H}), 3.90$ $3.75(\mathrm{~m}, 3 \mathrm{H}), 3.68-3.57(\mathrm{~m}, 1 \mathrm{H}), 3.31-3.22(\mathrm{~m}, 1 \mathrm{H}), 1.59-1.49(\mathrm{~m}, 4 \mathrm{H}), 0.88-0.81(\mathrm{~m}, 6 \mathrm{H})$;

${ }^{13} \mathrm{C}$ NMR $\left(150 \mathrm{MHz}\right.$, Acetone- $\left.d_{6}\right) \delta 162.2(\mathrm{~d}, J=244.4 \mathrm{~Hz}), 162.1(\mathrm{~d}, J=242.8 \mathrm{~Hz}), 141.0$, $134.1(\mathrm{~d}, J=61.2 \mathrm{~Hz}), 132.3(\mathrm{~d}, J=7.8 \mathrm{~Hz}), 126.6(\mathrm{~d}, J=7.9 \mathrm{~Hz}), 114.6(\mathrm{~d}, J=21.5 \mathrm{~Hz})$, $114.0(\mathrm{~d}, J=21.2 \mathrm{~Hz}), 67.5(\mathrm{~d}, J=6.7 \mathrm{~Hz}), 67.3(\mathrm{~d}, J=7.1 \mathrm{~Hz}), 61.5(\mathrm{~d}, J=10.7 \mathrm{~Hz}), 48.4(\mathrm{~d}$, $J=160.0 \mathrm{~Hz}), 43.7(\mathrm{~d}, J=5.4 \mathrm{~Hz}), 23.6(\mathrm{~d}, J=5.5 \mathrm{~Hz}), 23.5(\mathrm{~d}, J=5.9 \mathrm{~Hz}), 9.4,9.4 ;{ }^{19} \mathrm{~F}$ NMR (565 MHz, $\left.\mathrm{CDCl}_{3}\right) \delta-114.02,-114.69 ;{ }^{31} \mathrm{P}$ NMR (243 MHz, Acetone- $\left.d_{6}\right) \delta$ 23.01; HRMS (ESI): $m / z$ calcd. for $\mathrm{C}_{22} \mathrm{H}_{27} \mathrm{~F}_{2} \mathrm{~N}_{2} \mathrm{NaO}_{4} \mathrm{P}[\mathrm{M}+\mathrm{Na}]^{+}: 475.1569$, found: 475.1568 .

\subsection{Procedure for the preparation of $N$-benzoyl-5-hydroxy-pyridazin derivative 11}

Step 1: To a solution of $3 \mathbf{b c}(0.2 \mathrm{mmol}, 77.0 \mathrm{mg})$ in $\mathrm{CH}_{2} \mathrm{Cl}_{2}(3.0 \mathrm{~mL})$ were added benzoyl chloride (0.6 mmol, $72 \mathrm{uL}), N, N$-diisopropylethanamine $(0.52 \mathrm{mmol}, 86 \mathrm{uL})$ and DMAP $(0.02 \mathrm{mmol}, 2.4 \mathrm{mg})$ at $0{ }^{\circ} \mathrm{C}$. The mixture was stirred until the starting material disappeared. The reaction mixture was treated with acetic acid $(100 \mathrm{uL})$ and was purified by silica gel flash chromatography eluting with petroleum ether and ethyl acetate (v/v, 2:1) to afford the $N$-benzoylation product as a yellow oil (109.5 mg, $99 \%$ yield).

Step 2: $\mathrm{NaBH}_{4}(26.0 \mathrm{mg}, 0.68 \mathrm{mmol})$ was added to the solution of the above $N$-benzoylation product $(76.0 \mathrm{mg}, 0.137 \mathrm{mmol})$ in $\mathrm{THF} / \mathrm{MeOH}(1.5 / 0.5 \mathrm{~mL})$ at $-30{ }^{\circ} \mathrm{C}$. The reaction mixture was stirred until the starting material was disappeared after $3 \mathrm{~h}$. The reaction solution was treated with acetic acid $(100 \mathrm{uL})$ and purified by column chromatography on silica gel to give the reduction product $11(66.3 \mathrm{mg}, 87 \%$ yield, $17: 1 d r)$ as a colorless oil.

Dipropyl ((3S,4R,5R)-2-benzoyl-4,6-bis(4-fluorophenyl)-5-hydroxy-2,3,4,5-tetrahydro-p yridazin-3-yl)phosphonate (11).

Colorless oil; $[\alpha]_{\mathrm{D}}^{20}=+216.1^{\circ}(c=1.0, \mathrm{DCM})$; HPLC $\left(\mathrm{IC}, n\right.$-hexane $/^{i} \mathrm{PrOH}=80 / 20$, flow rate $=0.5 \mathrm{~mL} / \mathrm{min}, \mathrm{I}=254 \mathrm{~nm}$ ): $t_{\mathrm{R}}=33.5 \mathrm{~min}$ (major), $25.2 \min$ (minor); ${ }^{1} \mathrm{H}$ NMR $(600 \mathrm{MHz}$, Acetone- $\left.d_{6}\right) \delta 7.94(\mathrm{dd}, J=8.7,5.5 \mathrm{~Hz}, 2 \mathrm{H}), 7.73(\mathrm{~d}, J=7.5 \mathrm{~Hz}, 2 \mathrm{H}), 7.57(\mathrm{t}, J=7.4 \mathrm{~Hz}, 1 \mathrm{H})$, $7.52(\mathrm{t}, J=7.5 \mathrm{~Hz}, 2 \mathrm{H}), 7.22(\mathrm{dd}, J=8.6,5.2 \mathrm{~Hz}, 2 \mathrm{H}), 7.15(\mathrm{dt}, J=8.9,4.5 \mathrm{~Hz}, 4 \mathrm{H}), 6.51(\mathrm{~d}$, $J=11.7 \mathrm{~Hz}, 1 \mathrm{H}), 5.49(\mathrm{~d}, J=17.2 \mathrm{~Hz}, 1 \mathrm{H}), 4.56(\mathrm{~d}, J=11.5 \mathrm{~Hz}, 1 \mathrm{H}), 4.39-4.24(\mathrm{~m}, 2 \mathrm{H})$, $4.22-4.13(\mathrm{~m}, 1 \mathrm{H}), 4.12-4.02(\mathrm{~m}, 2 \mathrm{H}), 1.92-1.81(\mathrm{~m}, 2 \mathrm{H}), 1.73-1.60(\mathrm{~m}, 2 \mathrm{H}), 1.10(\mathrm{t}, J=7.4$ $\mathrm{Hz}, 3 \mathrm{H}), 0.91(\mathrm{t}, J=7.4 \mathrm{~Hz}, 3 \mathrm{H}) ;{ }^{13} \mathrm{C}$ NMR $\left(150 \mathrm{MHz}\right.$, Acetone- $\left.d_{6}\right) \delta 169.4,163.5(\mathrm{~d}, J=$ $248.0 \mathrm{~Hz}), 162.0(\mathrm{~d}, J=244.9 \mathrm{~Hz}), 148.3,136.7(\mathrm{dd}, J=17.6,3.3 \mathrm{~Hz}), 134.6,132.2(\mathrm{~d}, J=$ $3.2 \mathrm{~Hz}), 130.6,129.5,129.0(\mathrm{~d}, J=8.4 \mathrm{~Hz}), 128.6(\mathrm{~d}, J=8.1 \mathrm{~Hz}), 127.6,115.8(\mathrm{~d}, J=21.7$ $\mathrm{Hz}), 115.0(\mathrm{~d}, J=21.9 \mathrm{~Hz}), 69.5(\mathrm{~d}, J=7.2 \mathrm{~Hz}), 68.8(\mathrm{~d}, J=7.4 \mathrm{~Hz}), 61.5,48.1(\mathrm{~d}, J=154.0$ 
$\mathrm{Hz}), 44.0(\mathrm{~d}, J=5.4 \mathrm{~Hz}), 23.7(\mathrm{~d}, J=2.4 \mathrm{~Hz}), 23.7(\mathrm{~d}, J=2.7 \mathrm{~Hz}), 9.5,9.3 ;{ }^{19} \mathrm{~F}$ NMR $(565$

$\left.\mathrm{MHz}, \mathrm{CDCl}_{3}\right) \delta-111.09,-114.17 ;{ }^{31} \mathrm{P}$ NMR (243 MHz, Acetone- $\left.d_{6}\right) \delta 22.17$; HRMS (ESI): $m / z$ calcd. for $\mathrm{C}_{29} \mathrm{H}_{31} \mathrm{~F}_{2} \mathrm{~N}_{2} \mathrm{NaO}_{5} \mathrm{P}[\mathrm{M}+\mathrm{Na}]^{+}:$579.1831, found: 579.1837.

\section{X-ray crystal structure of 3aa}

Recrystallization of the product 3aa from $\mathrm{CH}_{2} \mathrm{Cl}_{2}$ /hexane afforded crystals suitable for diffraction analysis. The absolute configuration 3aa was determined to be $(5 R, 6 S)$ by $\mathrm{X}$-ray diffraction analysis and thus, the absolute configuration of others was deduced.

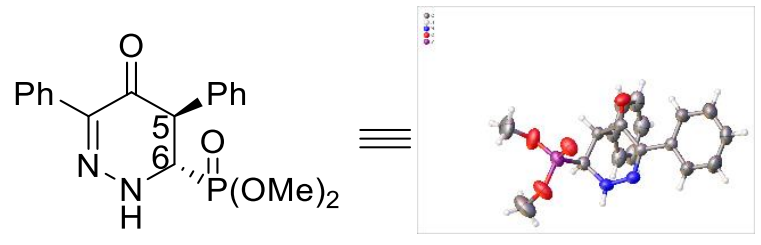

X-ray crystal structure of 3aa shown with 30\% thermal ellipsoids.

\begin{tabular}{|c|c|}
\hline \multicolumn{2}{|c|}{ Table S3: Crystal data and structure refinement for 3aa. } \\
\hline Empirical formula & $\mathrm{C}_{18} \mathrm{H}_{19} \mathrm{~N}_{2} \mathrm{O}_{4} \mathrm{P}$ \\
\hline Formula weight & 358.32 \\
\hline Temperature/K & $291.32(10)$ \\
\hline Crystal system & orthorhombic \\
\hline Space group & $\mathrm{P} 2{ }_{1} 2_{1} 2_{1}$ \\
\hline $\mathrm{a} / \AA$ & $8.84457(13)$ \\
\hline $\mathrm{b} / \AA$ & $11.92743(14)$ \\
\hline $\mathrm{c} / \AA$ & $16.87678(16)$ \\
\hline$\alpha /^{\circ}$ & 90 \\
\hline$\beta /{ }^{\circ}$ & 90 \\
\hline$\gamma /{ }^{\circ}$ & 90 \\
\hline Volume $/ \AA^{3}$ & $1780.38(4)$ \\
\hline $\mathrm{Z}$ & 4 \\
\hline$\rho_{\text {calc }} \mathrm{g} / \mathrm{cm}^{3}$ & 1.337 \\
\hline$\mu / \mathrm{mm}^{-1}$ & 1.589 \\
\hline $\mathrm{F}(000)$ & 752.0 \\
\hline Crystal size/mm $\mathrm{mm}^{3}$ & $0.2 \times 0.2 \times 0.2$ \\
\hline Radiation & $\operatorname{CuK} \alpha(\lambda=1.54184)$ \\
\hline $2 \Theta$ range for data collection ${ }^{\circ}$ & 9.08 to 143.646 \\
\hline Index ranges & $-9 \leq \mathrm{h} \leq 10,-14 \leq \mathrm{k} \leq 14,-20 \leq 1 \leq 20$ \\
\hline Reflections collected & 9879 \\
\hline Independent reflections & $3426\left[\mathrm{R}_{\mathrm{int}}=0.0278, \mathrm{R}_{\text {sigma }}=0.0281\right]$ \\
\hline
\end{tabular}




\begin{tabular}{ll}
\hline Data/restraints/parameters & $3426 / 36 / 204$ \\
Goodness-of-fit on $\mathrm{F}^{2}$ & 1.051 \\
Final R indexes [I $>=2 \sigma(\mathrm{I})]$ & $\mathrm{R}_{1}=0.0492, \mathrm{wR}_{2}=0.1327$ \\
Final R indexes [all data] & $\mathrm{R}_{1}=0.0503, \mathrm{wR}_{2}=0.1340$ \\
Largest diff. peak/hole / e $\AA^{-3}$ & $0.52 /-0.38$ \\
Flack parameter & $-0.007(11)$ \\
\hline
\end{tabular}

\section{Procedure for catalyst}
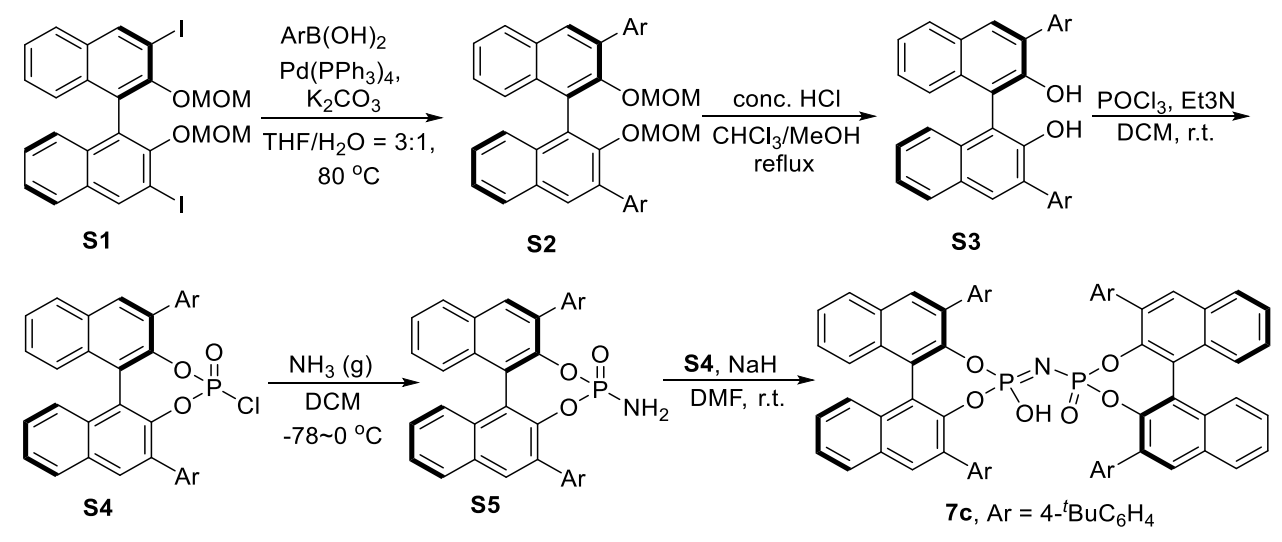

\subsection{Synthesis of $(R)$-1, 10-binaphthyl-3, $3^{\prime}$-diaryl phosphoryl chloride $S 4^{[1]}$}

The starting BINOL derivative $13(2.06 \mathrm{~g}, 3.74 \mathrm{mmol})$ was dissolved in dry dichloromethane $(14.4 \mathrm{~mL}), \mathrm{POCl}_{3}(2.0$ equivalents, $0.7 \mathrm{~mL}, 7.48 \mathrm{mmol})$ and triethylamine (3 equiv., $1.6 \mathrm{~mL}, 11.22 \mathrm{mmol}$ ) was successively added dropwise, then the reaction was stirred at room temperature overnight. The crude mixture was purified by column chromatography ( $\mathrm{PE} / \mathrm{EA} / \mathrm{DCM}=8 / 1 / 1)$ delivered product 13 as a white solid (2.22 $\mathrm{g}, 94 \%$ yield).

\subsection{Synthesis of compound S5}

The compound $\mathbf{S 4}(1.0 \mathrm{~g}, 1.58 \mathrm{mmol})$ was dissolved in dry dichloromethane $(6 \mathrm{~mL} / \mathrm{mmol})$ and then cooled to $-78{ }^{\circ} \mathrm{C}$, anhydrous ammonia gas was condensed into the reaction flask (about $10 \mathrm{~min}$, when a thin layer of liquid ammonia was seen in the reaction flask, $\sim 2 \mathrm{~mm}$ ), then warmed up slowly to room temperature within the cold bath. Purification of the crude mixture by column chromatography $\left(\mathrm{CH}_{2} \mathrm{Cl}_{2}\right)$ delivered product $\mathbf{S 5}$ as a white solid (764 mg, $79 \%$ yield).

\subsection{Synthesis of imidodiphosphoric acid $7 \mathrm{c}^{[2]}$}

To a stirred solution of $(R)$-1, 10-binaphthyl-3, 3'-diaryl phosphoryl chloride $\mathbf{S 4}$ (820 mg, $1.3 \mathrm{mmol})$ in dry DMF $(10 \mathrm{~mL})$ was added $\mathrm{NaH}(60 \%$ in oil, $160 \mathrm{mg}, 4 \mathrm{mmol})$ under a nitrogen atmosphere at room temperature. After stirring for $5 \mathrm{~min},(R)-1,10$-binaphthyl-3, 
30-diaryl phosphoramide S5 (1 mmol) was added in one portion and the mixture was stirred for another $1 \mathrm{~h}$. After the reaction was complete, $30 \mathrm{~mL}$ of $\mathrm{CH}_{2} \mathrm{Cl}_{2}$ was added to the flask and the mixture was washed with $1 \mathrm{M} \mathrm{HCl}_{(\mathrm{aq})}$ and saturated brine. The organic layer was dried over $\mathrm{Na}_{2} \mathrm{SO}_{4}$ and concentrated under reduced pressure. The crude product was purified with silica gel column chromatography (PE/EA/ $\mathrm{CH}_{2} \mathrm{Cl}_{2}=8: 1: 1$ to $\left.3: 1: 1\right)$. The product was then successively dissolved in $\mathrm{CH}_{2} \mathrm{Cl}_{2}(20 \mathrm{~mL})$, acidified with $3 \mathrm{M} \mathrm{HCl}(20 \mathrm{~mL})$, washed with brine (20 $\mathrm{mL} \mathrm{X} \mathrm{2),} \mathrm{dried} \mathrm{over} \mathrm{Na}_{2} \mathrm{SO}_{4}$, and concentrated in vacuo to give catalyst $(R, R)-7 \mathbf{c}$ (788 mg, 65\% yield).

(11bR,11b'R)-4,4'-azanediylbis(2,6-bis(4-(tert-butyl)phenyl)dinaphtho[2,1-d:1',2'-f] [1,3,2 ]dioxaphosphepine-4-oxide) (7c). White solid; mp 241.6-243.1 ${ }^{\circ} \mathrm{C} ;[\alpha]_{\mathrm{D}}^{20}=-470.0^{\circ}(c=1.0$, DCM); ${ }^{1} \mathrm{H}$ NMR $\left(600 \mathrm{MHz}, \mathrm{CDCl}_{3}\right) \delta 8.13(\mathrm{~d}, J=8.2 \mathrm{~Hz}, 2 \mathrm{H}), 8.04(\mathrm{~s}, 2 \mathrm{H}), 7.96(\mathrm{~d}, J=8.2$ $\mathrm{Hz}, 2 \mathrm{H}), 7.83$ (s, 2H), 7.67 (t, $J=7.5 \mathrm{~Hz}, 2 \mathrm{H}), 7.61(\mathrm{~d}, J=8.5 \mathrm{~Hz}, 2 \mathrm{H}), 7.54-7.46(\mathrm{~m}, 10 \mathrm{H})$, $7.40(\mathrm{~d}, J=8.1 \mathrm{~Hz}, 4 \mathrm{H}), 7.32(\mathrm{t}, J=7.7 \mathrm{~Hz}, 2 \mathrm{H}), 6.68(\mathrm{~d}, J=7.9 \mathrm{~Hz}, 4 \mathrm{H}), 6.56(\mathrm{~d}, J=7.9 \mathrm{~Hz}$, 4H), 6.14 (br, 1H), 6.13 (br, 1H), 1.32 (s, 18H), 0.95 (s, 18H); $\left.{ }^{13} \mathrm{C} \mathrm{NMR} \mathrm{(150} \mathrm{MHz,} \mathrm{CDCl}_{3}\right) \delta$ $150.5,149.9,145.1,144.8,134.7,134.0,133.6,132.1,132.0,131.8,131.6,130.8,130.7$, $130.5,129.8,128.9,128.6,128.4,127.5,127.0,126.4,126.3,126.1,125.7,125.0,124.5$, 123.4, 122.7, 34.6, 34.3, 31.3, 31.1; ${ }^{31} \mathrm{P}$ NMR (243 MHz, $\left.\mathrm{CDCl}_{3}\right) \delta 1.64 ; \mathrm{HRMS}$ (ESI): $\mathrm{m} / z$ calcd. for $\mathrm{C}_{80} \mathrm{H}_{73} \mathrm{NNaO}_{6} \mathrm{P}_{2}[\mathrm{M}-\mathrm{Na}]^{+}:$1228.4805, found: 1228.4853 .

\section{General procedure for preparation of $\alpha$-haloketones}

$\alpha$-Haloketones were prepared from bisbenzyl ketones, which were prepared as fellows.

\subsection{Preparation of 1, 3-diarylpropan-2-ones}

General Procedure for bisbenzyl ketones from phenylacetic acids. ${ }^{[3]}$
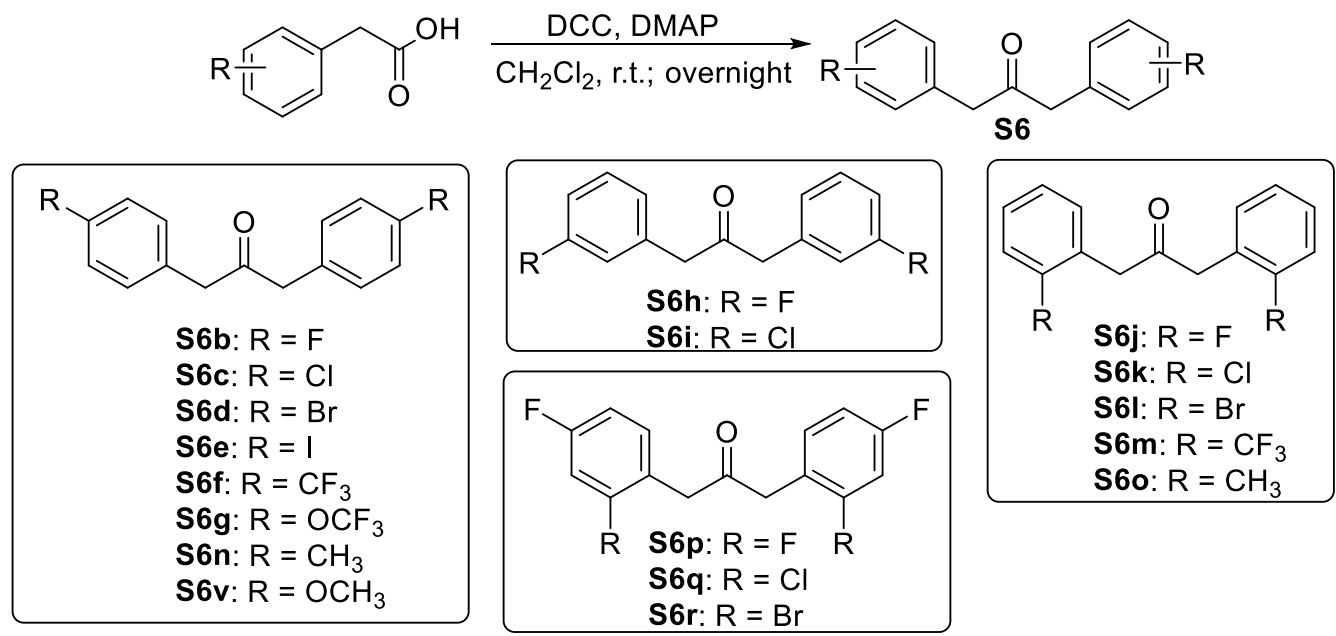

The general bisbenzyl ketone was prepared by adding substituted phenylacetic acid (1 
equiv) to a stirring solution of DCC (1.05 equiv) and DMAP (0.25 equiv) in dry $\mathrm{CH}_{2} \mathrm{Cl}_{2}$ at room temperature. Reaction mixture was kept for $24 \mathrm{~h}$. at room temperature (It was warmed up appropriately and $\mathrm{ClCH}_{2} \mathrm{CH}_{2} \mathrm{Cl}$ or THF was used as solvent if necessary.) and then filtered through Celite. The filtrate was distilled off and the residue was purified by flash chromatography on silica gel.

\section{Dibenzylketone S6s were prepared from 4-fluoro-2-methylbenzoic acid.}

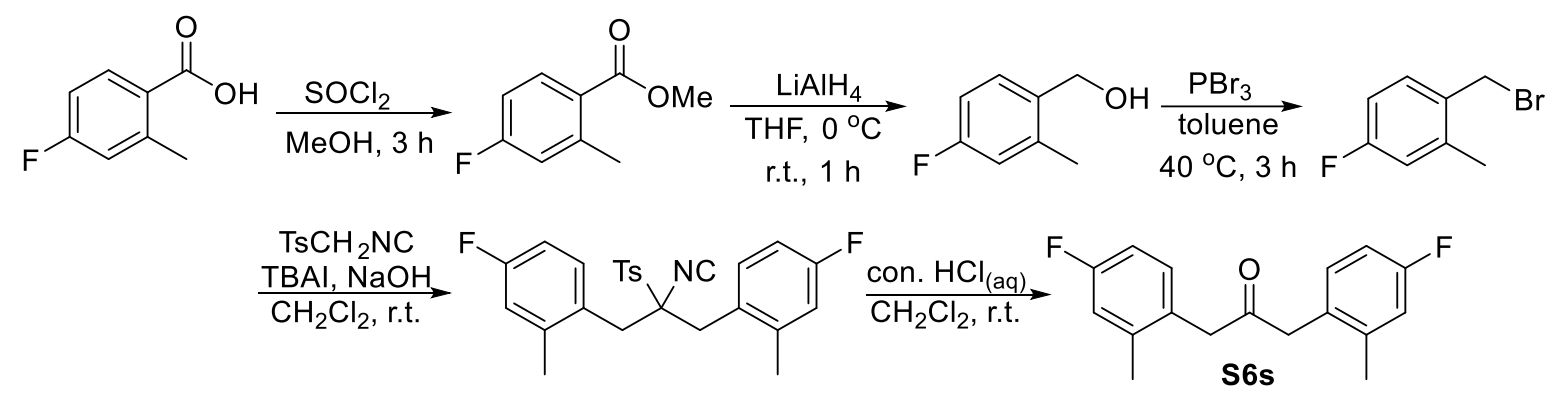

To a stirred solution of 2-methyl-4-fluoro-benzoic acid (3.95 g, $25.6 \mathrm{mmol})$ in methanol (16 $\mathrm{mL}$ ) was added thionyl chloride $(3.8 \mathrm{~mL}, 51.2 \mathrm{mmol})$ dropwise at $0{ }^{\circ} \mathrm{C}$. Then the mixture solution was refluxed for 3 hours before cooling to room temperature. Then the reaction mixture was concentrated in vacuo to afford methyl 4-fluoro-2-methylbenzoate as colorless oil, quantitatively. A solution of the methyl benzoate in $25 \mathrm{ml}$ of THF was cooled to $0{ }^{\circ} \mathrm{C}$, treated with $\mathrm{LiAlH}_{4}(1.23 \mathrm{~g}, 30.72 \mathrm{mmol})$, and allowed to warm to room temperature over 1 hour. Reaction quenched sequentially with $1.3 \mathrm{ml}$ of water, $1.3 \mathrm{ml}$ of $1 \mathrm{~N} \mathrm{NaOH}$, and $4 \mathrm{ml}$ of water and left to stir for thirty minutes. Reaction was filtered and the filtrate concentrated to afford a crude product ( $3.23 \mathrm{~g}, 90 \%$ yield) which was purified by silica gel chromatography (PE/EA =2/1), a colorless oil. Phosphorous tribromide (320 uL) was added to a stirred solution of the 4-fluoro-2-methylbenzyl alcohol $(9.35 \mathrm{mmol}, 1.31 \mathrm{~g})$ in toluene $(13 \mathrm{ml})$ at $40{ }^{\circ} \mathrm{C}$. The solution was heated to $100^{\circ} \mathrm{C}$ for $30 \mathrm{~min}$, and the reaction was cooled to ambient temperature. The liquid was decanted and washed with water $(2 \times 50 \mathrm{ml})$ and brine $(50 \mathrm{ml})$. The combined aqueous layer was extracted with diethyl ether $(70 \mathrm{ml})$ and the combined organic layer was evaporated to yield a semisolid residue. The residue was dissolved in diethyl ether $(350 \mathrm{ml})$ and washed with water $(2 \times 100 \mathrm{ml})$ and brine $(100 \mathrm{ml})$. The organic phase was dried over $\mathrm{Na}_{2} \mathrm{SO}_{4}$, filtered and evaporated to afford 4-fluoro-2-methylbenzyl bromide as pale-yellow oil (1.80 g, 95\% yield).

Dibenzylketones were obtained according to a procedure developed by Van Leusen and collegues. ${ }^{[4]}$ Using 4-methylbenzenesulfonylmethyl isocyanide (TosMIC) and the substituted benzyl bromide in phase transfer catalysis conditions. TosMIC (0.9 g, $4.5 \mathrm{mmol}, 1.0$ equiv.), 
the benzyl bromide ( 9 mmol, 2.0 equiv.) and $0.37 \mathrm{~g}$ TBAI (tetrabutylammonium iodide, 1 mmol, 0.1 equiv.) were stirred vigorously in $90 \mathrm{~mL} \mathrm{CH}_{2} \mathrm{Cl}_{2}$ for $10 \mathrm{~min}$ before $45 \mathrm{~mL}$ aqueous $\mathrm{NaOH}$ (40 wt \% in $\mathrm{H}_{2} \mathrm{O}$ ) was added. Vigorous stirring was carried on overnight. The mixture was then neutralized with $\mathrm{HCl}(10 \mathrm{wt} \%)$ and the organic layer was collected. The aqueous layer was extracted 3 times with $\mathrm{CH}_{2} \mathrm{Cl}_{2}$ and the organic layers were dried over anhydrous $\mathrm{Na}_{2} \mathrm{SO}_{4}$. Evaporation of the solvent gave the crude dibenzyl isocyanide which was eventually dissolved in $\mathrm{CH}_{2} \mathrm{Cl}_{2}(50 \mathrm{~mL})$ and $\mathrm{HCl}(37 \mathrm{wt} \%, 10 \mathrm{~mL})$ and vigorously stirred for $12 \mathrm{~h}$ at room temperature. The mixture was neutralized with saturated $\mathrm{NaHCO}_{3}$. The organic layer was collected, the aqueous layer was extracted three times with $\mathrm{CH}_{2} \mathrm{Cl}_{2}$ and the organic layers were dried over $\mathrm{Na}_{2} \mathrm{SO}_{4}$; removal of the solvent under vacuum gave a crude product which

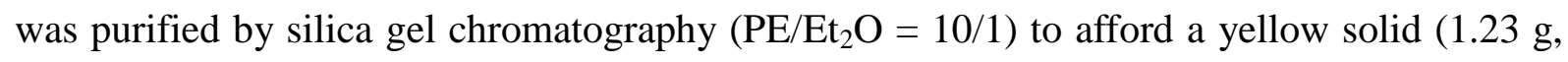
$97 \%$ yield).

\section{Preparation of 1-(4-fluorophenyl)-3-(o-tolyl)propan-2-one (S6t). ${ }^{[5]}$}

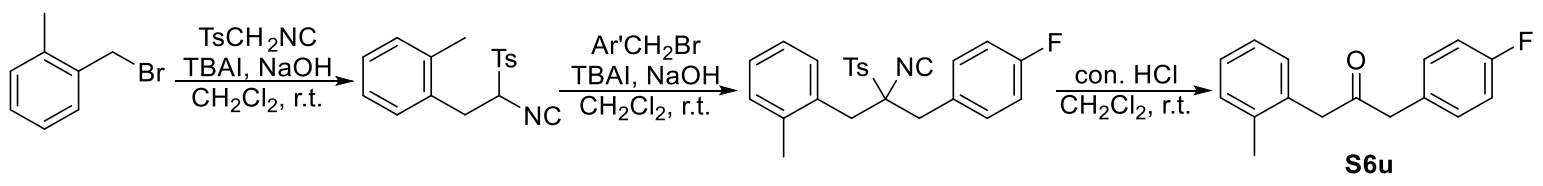

A mixture of $\mathrm{n}-\mathrm{Bu}_{4} \mathrm{NI}(250 \mathrm{mg}), \mathrm{NaOH}(6.0 \mathrm{~g})$ dissolved in $15 \mathrm{~mL}$ of water, and $\mathrm{CH}_{2} \mathrm{Cl}_{2}(25 \mathrm{~mL})$ was cooled in an ice bath with stirring. To the mixture was added in one portion a $\mathrm{CH}_{2} \mathrm{Cl}_{2}(25 \mathrm{~mL})$ solution of TosMIC $12(3.61 \mathrm{~g}, 18.5 \mathrm{mmol})$. After $30 \mathrm{~min}$, 2-methylbenzyl bromide $(1.5 \mathrm{~mL}, 10.94 \mathrm{mmol})$ dissolved in $\mathrm{CH}_{2} \mathrm{Cl}_{2}(20 \mathrm{~mL})$ was added in one portion. The mixture was stirred for $1 \mathrm{~h}$ in the ice bath, then at room temperature for $4 \mathrm{~h}$. The reaction mixture was washed with water and concentrated to a volume of ca. $20 \mathrm{~mL}$. The concentrate was diluted with $\mathrm{MeOH}$ (ca. $100 \mathrm{~mL}$ ) and stored in a freezer. The precipitate was collected by filtration and washed with $\mathrm{MeOH}$. Chromatography of the crude product with silica gel $\left(\mathrm{PE} / \mathrm{CH}_{2} \mathrm{Cl}_{2}=1 / 1\right)$ afforded pure 1-(2-isocyano-2-tosylethyl)-2-methyl benzene (3.1 $\mathrm{g}, 95 \%)$ as white powder.

A $100 \mathrm{ml}$ round-bottomed flask equipped with a magnetic stir bar was charged with the 1-(2-isocyano-2-tosylethyl)-2-methyl benzene (1.5 g, $5 \mathrm{mmol}$ ), 4-fluoro-benzylbromide (5.5 mmol, 1.10 equiv.), and $\mathrm{Bu}_{4} \mathrm{NI}(1.0 \mathrm{mmol}, 370 \mathrm{mg})$, then $\mathrm{CH}_{2} \mathrm{Cl}_{2}(25 \mathrm{~mL})$ and $30 \% \mathrm{NaOH}$ aqueous solution $(25 \mathrm{~mL})$ were added under ice-water bath condition. After vigorous stirring for $3 \mathrm{~h}$ at room temperature, the mixture was poured into $\mathrm{H}_{2} \mathrm{O}(15 \mathrm{~mL})$, and was extracted with $\mathrm{CH}_{2} \mathrm{Cl}_{2}(3 \times 15 \mathrm{~mL})$, dried over $\mathrm{Na}_{2} \mathrm{SO}_{4}$, and concentrated to about $50 \mathrm{~mL}$ under reduced pressure with the aid of a rotary evaporator. The crude product was added $\mathrm{HCl}(37 \mathrm{wt} \%, 10$ 
$\mathrm{mL}$ ) and vigorously stirred for $12 \mathrm{~h}$ at room temperature. The mixture was neutralized with saturated $\mathrm{NaHCO}_{3}$. The organic layer was collected, the aqueous layer was extracted three times with $\mathrm{CH}_{2} \mathrm{Cl}_{2}$ and the organic layers were dried over $\mathrm{Na}_{2} \mathrm{SO}_{4}$; removal of the solvent under vacuum gave a crude product which was purified by silica gel chromatography $\left(\mathrm{PE} / \mathrm{Et}_{2} \mathrm{O}=10 / 1\right)$, as pale-yellow oil $(1.0 \quad \mathrm{~g}, \quad 83 \%$ yield $)$. 1-(4-fluorophenyl)-3-(o-tolyl)propan-2-one (S6u). ${ }^{1} \mathrm{H}$ NMR $\left(600 \mathrm{MHz}, \mathrm{CDCl}_{3}\right) \delta 7.22-7.16$ $(\mathrm{m}, 3 \mathrm{H}), 7.12-7.08(\mathrm{~m}, 3 \mathrm{H}), 7.02-6.98(\mathrm{~m}, 2 \mathrm{H}), 3.75(\mathrm{~s}, 2 \mathrm{H}), 3.68(\mathrm{~s}, 2 \mathrm{H}), 2.16(\mathrm{~s}, 3 \mathrm{H}) ;{ }^{13} \mathrm{C}$ NMR (150 MHz, $\left.\mathrm{CDCl}_{3}\right) \delta 205.3,162.0$ (d, $\left.J=245.7 \mathrm{~Hz}\right), 136.9,132.7,131.0,130.9,130.5$ $(\mathrm{d}, J=11.7 \mathrm{~Hz}), 129.7$ (d, $J=3.3 \mathrm{~Hz}), 127.5,126.2,115.5(\mathrm{~d}, J=21.5 \mathrm{~Hz}), 47.9,47.6,19.5$.

Preparation of 1-(4-fluorophenyl)-3-(4-methoxyphenyl)propan-2-one (S6u).
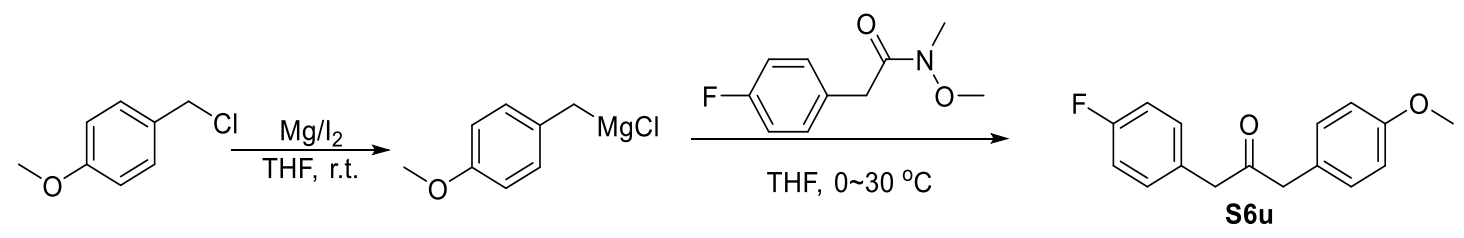

To a suspension of $478 \mathrm{mg}$ (19.9 mmol) of freshly crushed magnesium turnings and a small amount of iodine in $8 \mathrm{~mL}$ of anhydrous THF was slowly added a solution of $1.56 \mathrm{~g}(9.95$ mmol) of 4-methoxybenzyl chloride in $2 \mathrm{~mL}$ of anhydrous THF. After vigorous stirring for 2 $\mathrm{h}$ at room temperature, the reaction was completed and then a solution of $790 \mathrm{mg}(4 \mathrm{mmol})$ of 2-(4-fluorophenyl)- $N$-methoxy- $N$-methyl-acetamide in $3 \mathrm{~mL}$ of anhydrous THF was slowly added while the temperature was maintained at $0{ }^{\circ} \mathrm{C}$. After being stirred at room temperature for 24 hours, the reaction mixture was poured into a saturated ammonium chloride solution and extracted with ethyl ether and the organic extracts were dried. The solvent was removed under reduced pressure and the residual oil was purified by flash chromatography (PE/DCM = 3:1 to 1:1) to afford 1-(4-fluorophenyl)-3-(4-methoxyphenyl)-propan-2-one (S6u, $920 \mathrm{mg}$, $89 \%$, yellow oil). 


\subsection{General procedure for preparation of 1-halo-1, 3-diarylpropan-2-ones 1a-v.}

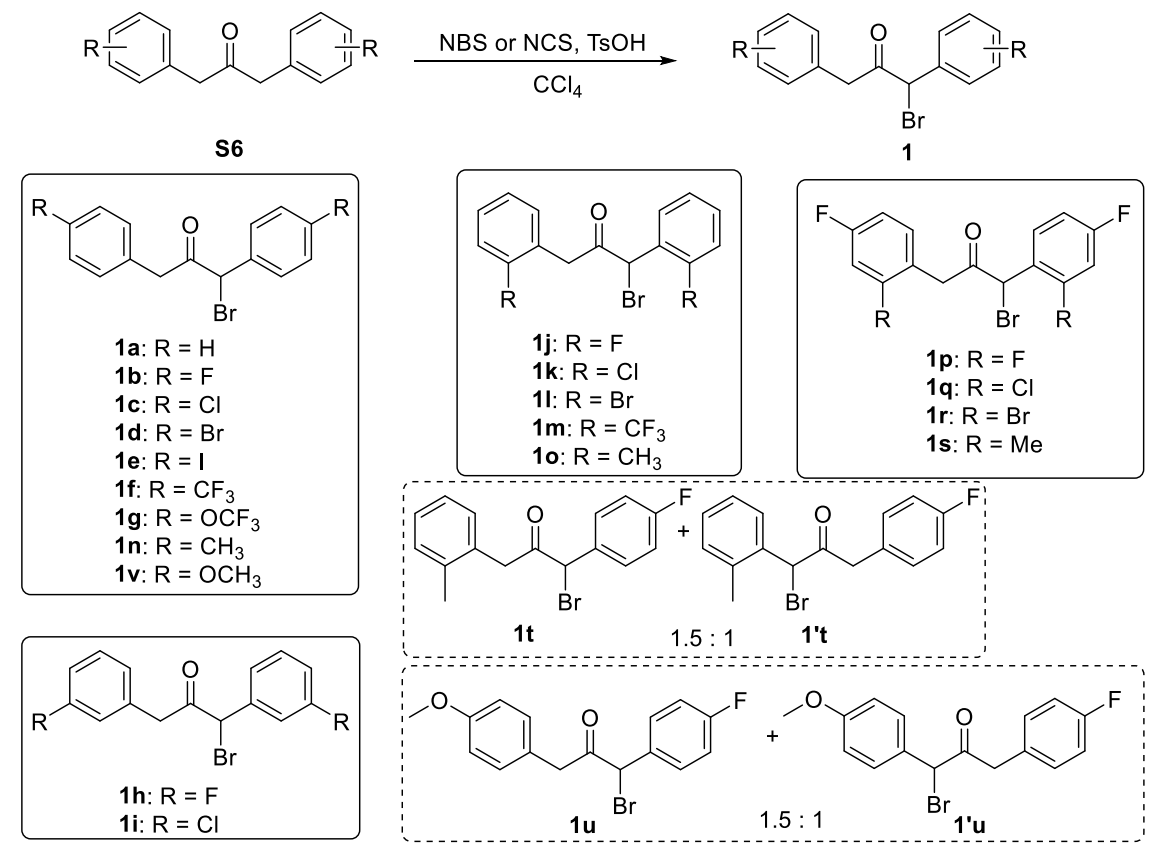

p-Toluenesulfonic acid monohydrate $\left(\mathrm{TsOH} \cdot \mathrm{H}_{2} \mathrm{O}, 0.1\right.$ equiv.) was added to a solution of 1,3-diarylpropan-2-one S6 (1 equiv.) and 1.2 equiv. $\mathrm{N}$-bromosuccinimide (NBS) in $\mathrm{CCl}_{4}$ (1M for S6) at room temperature under an argon atmosphere. The mixture was stirred at $30{ }^{\circ} \mathrm{C}$ until the starting material was disappeared. (It was warmed up appropriately if necessary.) The reaction mixture was purified directly by column chromatography to afford the 1-halo-1, 3-diarylpropan-2-one 1. The product was recrystallized from diethyl ether-petroleum if necessary. Notice: this 1-halo-1, 3-diarylpropan-2-ones are sensitive to natural light and need to be preserved in dark place at low temperature.

\section{Characterization of products}

Dimethyl ((3S, 4R)-5-oxo-4,6-diphenyl-2,3,4,5-tetrahydropyridazin-3-yl)phosphonate (3aa)

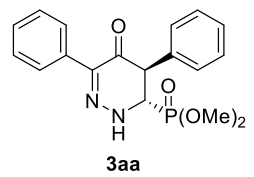

Isolated by silica gel column chromatography (petroleum ether : acetone $=$ $8: 1 \sim 1: 1, \mathrm{v} / \mathrm{v})$ to afford a yellow solid $(35.1 \mathrm{mg}, 98 \%$ yield, $81 \%$ ee, $>99: 1$ $d r), \mathrm{mp} 158.6-159.5^{\circ} \mathrm{C} ;[\alpha]_{\mathrm{D}}^{20}=-296.6^{\circ}\left(c=1.0, \mathrm{CHCl}_{3}\right)$; HPLC (Chiralpak ASH, $n$-hexane $/ \mathrm{PrOH}=70 / 30$, flow rate $=0.5 \mathrm{~mL} / \mathrm{min}, \mathrm{I}=254 \mathrm{~nm}$ ): $t_{\mathrm{R}}=25.6 \mathrm{~min}$ (major), 30.1 min (minor); ${ }^{1} \mathrm{H}$ NMR (600 MHz, $\left.\mathrm{CDCl}_{3}\right) \delta 7.70(\mathrm{~d}, J=7.5 \mathrm{~Hz}, 2 \mathrm{H}), 7.57$ (brs, $\left.1 \mathrm{H}\right)$, 7.39-7.28 (m, 8H), $4.42(\mathrm{dd}, J=12.8,7.1 \mathrm{~Hz}, 1 \mathrm{H}), 4.07(\mathrm{dd}, J=11.3,7.1 \mathrm{~Hz}, 1 \mathrm{H}), 3.69$ (d, $J$ $=11.1 \mathrm{~Hz}, 3 \mathrm{H}), 3.66(\mathrm{~d}, J=11.1 \mathrm{~Hz}, 3 \mathrm{H}),{ }^{13} \mathrm{C} \mathrm{NMR}\left(150 \mathrm{MHz}, \mathrm{CDCl}_{3}\right) \delta 184.1(\mathrm{~d}, J=5.1$ Hz), 139.7, $135.4(\mathrm{~d}, J=12.9 \mathrm{~Hz}), 133.9,129.0,128.3,128.2,128.1,128.0,127.7,57.9$ (d, $J$ $=150.8 \mathrm{~Hz}), 53.8(\mathrm{~d}, J=7.0 \mathrm{~Hz}), 53.3(\mathrm{~d}, J=7.2 \mathrm{~Hz}), 49.6(\mathrm{~d}, J=5.4 \mathrm{~Hz}) ;{ }^{31} \mathrm{P}$ NMR $(243$ 
$\left.\mathrm{MHz}, \mathrm{CDCl}_{3}\right) \delta$ 22.00; HRMS (ESI): $\mathrm{m} / z$ calcd. for $\mathrm{C}_{18} \mathrm{H}_{19} \mathrm{~N}_{2} \mathrm{NaO}_{4} \mathrm{P}[\mathrm{M}+\mathrm{Na}]^{+}: 381.0975$, found, 381.0975 .

\section{Diethyl ((3S, 4R)-5-oxo-4,6-diphenyl-2,3,4,5-tetrahydropyridazin-3-yl)phosphonate (3ab)}

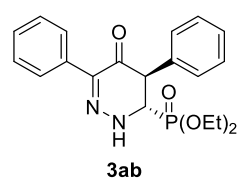

Isolated by silica gel column chromatography (petroleum ether : acetone $=$ 8:1 1:1, v/v) to afford a yellow solid (33.6 mg, 87\% yield, 94\% ee, >99:1 $d r)$, mp 133.5-135.0 ${ }^{\circ} \mathrm{C} ;[\alpha]_{\mathrm{D}}^{20}=-265.6^{\circ}(c=1.0$, DCM); HPLC (Chiralpak ASH, $n$-hexane $/ \mathrm{PrOH}=70 / 30$, flow rate $=0.5 \mathrm{~mL} / \mathrm{min}, \mathrm{I}=254 \mathrm{~nm}$ ): $t_{\mathrm{R}}=16.9 \mathrm{~min}$ (major), $23.1 \mathrm{~min}$ (minor); ${ }^{1} \mathrm{H}$ NMR (600 MHz, $\left.\mathrm{CDCl}_{3}\right) \delta 7.77(\mathrm{~s}, 1 \mathrm{H}), 7.70(\mathrm{~d}, J=7.5 \mathrm{~Hz}, 2 \mathrm{H}), 7.37-$ $7.27(\mathrm{~m}, 8 \mathrm{H}), 4.37$ (dd, $J=12.9,6.6 \mathrm{~Hz}, 1 \mathrm{H}), 4.16-4.08(\mathrm{~m}, 1 \mathrm{H}), 4.10-3.96(\mathrm{~m}, 4 \mathrm{H}), 1.27-$ $1.19(\mathrm{~m}, 6 \mathrm{H}) ;{ }^{13} \mathrm{C}$ NMR $\left(150 \mathrm{MHz}, \mathrm{CDCl}_{3}\right) \delta 184.1(\mathrm{~d}, J=4.6 \mathrm{~Hz}), 139.30,135.8(\mathrm{~d}, J=13.1$ $\mathrm{Hz}), 134.1,129.0,128.3,128.1,128.0,128.0,127.7,63.5$ (d, $J=7.1 \mathrm{~Hz}), 63.0$ (d, $J=7.2 \mathrm{~Hz})$, $58.1(\mathrm{~d}, J=150.7 \mathrm{~Hz}), 49.6,16.4(\mathrm{~d}, J=5.7 \mathrm{~Hz}), 16.2(\mathrm{~d}, J=5.7 \mathrm{~Hz}) ;{ }^{31} \mathrm{P}$ NMR $(243 \mathrm{MHz}$, $\left.\mathrm{CDCl}_{3}\right) \delta$ 19.69; HRMS (ESI): $\mathrm{m} / z$ calcd. for $\mathrm{C}_{20} \mathrm{H}_{23} \mathrm{~N}_{2} \mathrm{NaO}_{4} \mathrm{P}[\mathrm{M}+\mathrm{Na}]^{+}:$409.1288, found: 409.1292 .

Dipropyl $\quad((3 S, \quad 4 R)-5-0 x 0-4,6-d i p h e n y l-2,3,4,5$-tetrahydropyridazin-3-yl)phosphonate (3ac)

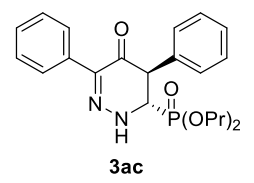

Isolated by silica gel column chromatography (petroleum ether : acetone $=$ 8:1 1:1, v/v) to afford a yellow oil (33.9 mg, 82\% yield, 96\% ee, >99:1 dr); $[\alpha]_{\mathrm{D}}^{20}=-213.6^{\circ}(c=1.0, \mathrm{DCM})$; HPLC (Chiralpak ASH, $n$-hexane $/ \mathrm{PrOH}=$ $70 / 30$, flow rate $=0.5 \mathrm{~mL} / \mathrm{min}, \mathrm{I}=254 \mathrm{~nm}$ ): $t_{\mathrm{R}}=14.1 \mathrm{~min}$ (major), $17.5 \mathrm{~min}$ (minor); ${ }^{1} \mathrm{H}$ NMR (600 MHz, $\left.\mathrm{CDCl}_{3}\right) \delta$ 7.73-7.68 (m, 3H), 7.38-7.26 (m, 8H), 4.38 (dd, $J=13.2,6.8 \mathrm{~Hz}$, $1 \mathrm{H}), 4.07(\mathrm{dd}, J=11.4,6.8 \mathrm{~Hz}, 1 \mathrm{H}), 4.02(\mathrm{dq}, J=9.8,6.8 \mathrm{~Hz}, 1 \mathrm{H}), 3.94(\mathrm{q}, J=6.8 \mathrm{~Hz}, 2 \mathrm{H})$, $3.89(\mathrm{dt}, J=9.7,7.1 \mathrm{~Hz}, 1 \mathrm{H}), 1.66-1.53(\mathrm{~m}, 4 \mathrm{H}), 0.88(\mathrm{t}, J=7.4 \mathrm{~Hz}, 3 \mathrm{H}), 0.87$ (t, $J=7.3 \mathrm{~Hz}$, $7 \mathrm{H}) ;{ }^{13} \mathrm{CNMR}\left(151 \mathrm{MHz}, \mathrm{CDCl}_{3}\right) \delta 184.1(\mathrm{~d}, J=4.8 \mathrm{~Hz}), 139.3,135.9(\mathrm{~d}, J=13.0 \mathrm{~Hz}), 134.1$, 129.0, 128.3, 128.1, 128.0, 127.9, 127.7, 68.9 (d, $J=7.3 \mathrm{~Hz}), 68.5(\mathrm{~d}, J=7.6 \mathrm{~Hz}), 58.2(\mathrm{~d}, J=$ $150.8 \mathrm{~Hz}), 49.7,23.9$ (d, $J=5.6 \mathrm{~Hz}), 23.7(\mathrm{~d}, J=6.0 \mathrm{~Hz}), 9.9,9.9 ;{ }^{31} \mathrm{P}$ NMR $(243 \mathrm{MHz}$, $\left.\mathrm{CDCl}_{3}\right) \delta$ 19.71; HRMS (ESI): $\mathrm{m} / z$ calcd. for $\mathrm{C}_{22} \mathrm{H}_{27} \mathrm{~N}_{2} \mathrm{NaO}_{4} \mathrm{P}[\mathrm{M}+\mathrm{Na}]^{+}:$437.1601, found: 437.1603 .

\section{Dipropyl ((3S,4R)-4,6-bis(4-fluorophenyl)-5-oxo-2,3,4,5-tetrahydropyridazin-3-yl)phos- phonate (3bc)}

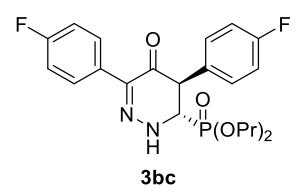

Isolated by silica gel column chromatography (petroleum ether : acetone $=$ $8: 1 \sim 1: 1, \mathrm{v} / \mathrm{v})$ to afford a yellow solid $(33.3 \mathrm{mg}, 74 \%$ yield, $99 \%$ $e e,>99: 1 d r), \operatorname{mp} 112.7-114.0^{\circ} \mathrm{C} ;[\alpha]_{\mathrm{D}}^{20}=-241.7^{\circ}(c=1.0, \mathrm{DCM}) ; \mathrm{HPLC}$ 
(Chiralpak ASH, $n$-hexane $/{ }^{i} \mathrm{PrOH}=70 / 30$, flow rate $=0.5 \mathrm{~mL} / \mathrm{min}, \mathrm{I}=254 \mathrm{~nm}$ ): $t_{\mathrm{R}}=13.0 \mathrm{~min}$ (major), $17.6 \mathrm{~min}$ (minor); ${ }^{1} \mathrm{H} \mathrm{NMR}\left(600 \mathrm{MHz}, \mathrm{CDCl}_{3}\right) \delta 7.74(\mathrm{~s}, 1 \mathrm{H}), 7.72-7.68(\mathrm{~m}, 2 \mathrm{H})$, 7.30-7.24 (m, 2H), 7.07-6.98 (m, 4H), 4.33 (dd, $J=12.8,7.7 \mathrm{~Hz}, 1 \mathrm{H}), 4.08-3.99$ (m, 2H), $3.96-3.88(\mathrm{~m}, 3 \mathrm{H}), 1.66-1.53(\mathrm{~m}, 4 \mathrm{H}), 0.90-0.85(\mathrm{~m}, 6 \mathrm{H}) ;{ }^{13} \mathrm{C} \mathrm{NMR}\left(151 \mathrm{MHz}, \mathrm{CDCl}_{3}\right) \delta$ $183.9(\mathrm{~d}, J=5.6 \mathrm{~Hz}), 162.7(\mathrm{~d}, J=247.7 \mathrm{~Hz}), 162.6(\mathrm{~d}, J=247.5 \mathrm{~Hz}), 138.4,131.3(\mathrm{dd}, J=$ 12.3, 3.4 Hz), 130.1 (d, $J=8.2 \mathrm{~Hz}), 129.4$ (d, $J=8.1 \mathrm{~Hz}), 115.9$ (d, $J=21.6 \mathrm{~Hz}), 114.9$ (d, $J$ $=21.4 \mathrm{~Hz}), 69.0(\mathrm{~d}, J=7.1 \mathrm{~Hz}), 68.6(\mathrm{~d}, J=7.3 \mathrm{~Hz}), 58.1(\mathrm{~d}, J=151.5 \mathrm{~Hz}), 49.2(\mathrm{~d}, J=2.4$ $\mathrm{Hz}), 23.9(\mathrm{~d}, J=5.6 \mathrm{~Hz}), 23.7(\mathrm{~d}, J=5.9 \mathrm{~Hz}), 9.9,9.9 ;{ }^{19} \mathrm{~F} \mathrm{NMR}\left(565 \mathrm{MHz}, \mathrm{CDCl}_{3}\right) \delta$ -113.60, -113.69; ${ }^{31} \mathrm{P}$ NMR (243 MHz, $\mathrm{CDCl}_{3}$ ) $\delta$ 19.36; HRMS (ESI): $\mathrm{m} / \mathrm{z}$ calcd. for $\mathrm{C}_{22} \mathrm{H}_{25} \mathrm{~F}_{2} \mathrm{~N}_{2} \mathrm{NaO}_{4} \mathrm{P}[\mathrm{M}+\mathrm{Na}]^{+}:$473.1412, found: 473.1411 .

\section{Dipropyl ((3S, 4R)-4, 6-bis(4-chlorophenyl)-5-oxo-2,3,4,5-tetrahydropyridazin-3-yl)pho s-phonate (3cc)}

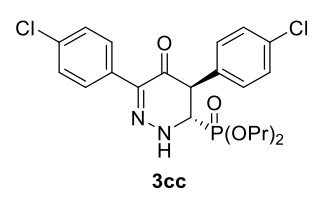

Isolated by silica gel column chromatography (petroleum ether : acetone $=8: 1 \sim 1: 1, \mathrm{v} / \mathrm{v})$ to afford a yellow solid $(47.3 \mathrm{mg}, 98 \%$ yield, $92 \%$ $e e,>99: 1 d r), \operatorname{mp} 39.2-40.6{ }^{\circ} \mathrm{C} ;[\alpha]_{\mathrm{D}}^{20}=-824.6^{\circ}(c=1.0, \mathrm{DCM}) ; \mathrm{HPLC}$

(Chiralpak IA, $n$-hexane $/{ }^{i} \mathrm{PrOH}=80 / 20$, flow rate $=0.5 \mathrm{~mL} / \mathrm{min}, \mathrm{I}=254 \mathrm{~nm}$ ): $t_{\mathrm{R}}=26.7 \mathrm{~min}$ (major), $20.1 \mathrm{~min}$ (minor); ${ }^{1} \mathrm{H}$ NMR (600 MHz, $\left.\mathrm{CDCl}_{3}\right) \delta 7.89(\mathrm{~s}, 1 \mathrm{H}), 7.70-7.62(\mathrm{~m}, 2 \mathrm{H})$, 7.34-7.30 (m, 2H), 7.30-7.26 (m, 2H), 7.23-7.19 (m, 2H), $4.33(\mathrm{dd}, J=13.3,7.5 \mathrm{~Hz}, 1 \mathrm{H})$, 4.05-3.97 (m, 2H), 3.97-3.87 (m, 3H), 1.64-1.53 (m, 4H), 0.90-0.84 (m, 6H); ${ }^{13} \mathrm{C}$ NMR (150 $\left.\mathrm{MHz}, \mathrm{CDCl}_{3}\right) \delta 183.5(\mathrm{~d}, J=5.4 \mathrm{~Hz}), 137.8,134.1(\mathrm{~d}, J=45.6 \mathrm{~Hz}), 133.9(\mathrm{~d}, J=12.5 \mathrm{~Hz})$, 132.4, 129.7, 129.1, 128.7, 128.1, 69.0 (d, $J=7.2 \mathrm{~Hz}), 68.7$ (d, $J=7.5 \mathrm{~Hz}), 57.9$ (d, $J=151.6$ $\mathrm{Hz}), 49.1,23.8(\mathrm{~d}, J=5.7 \mathrm{~Hz}), 23.7(\mathrm{~d}, J=5.9 \mathrm{~Hz}), 9.9,9.9 ;{ }^{31} \mathrm{P} \mathrm{NMR}\left(243 \mathrm{MHz}, \mathrm{CDCl}_{3}\right) \delta$ 19.20; HRMS (ESI): $m / z$ calcd. for $\mathrm{C}_{22} \mathrm{H}_{25} \mathrm{C}_{12} \mathrm{~N}_{2} \mathrm{NaO}_{4} \mathrm{P}$ [M+Na] $]^{+}: 505.0821$, found: 505.0814.

Dipropyl ((3S, 4R)-4,6-bis(4-bromophenyl)-5-oxo-2,3,4,5-tetrahydropyridazin-3-yl)phos -phonate (3dc)

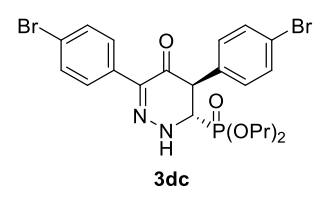

Isolated by silica gel column chromatography (petroleum ether : acetone $=8: 1 \sim 1: 1, \mathrm{v} / \mathrm{v})$ to afford a yellow solid $(57.2 \mathrm{mg}, 93 \%$ yield, $90 \%$ $e e,>99: 1 d r), \operatorname{mp} 71.3-73.1^{\circ} \mathrm{C} ;[\alpha]_{\mathrm{D}}^{20}=-167.5^{\circ}(c=1.0, \mathrm{DCM}) ; \mathrm{HPLC}$ (Chiralpak IA, $n$-hexane $/{ }^{i} \mathrm{PrOH}=80 / 20$, flow rate $\left.=0.5 \mathrm{~mL} / \mathrm{min}, \mathrm{I}=254 \mathrm{~nm}\right): t_{\mathrm{R}}=30.7 \mathrm{~min}$ (major), $22.4 \mathrm{~min}$ (minor); ${ }^{1} \mathrm{H} \mathrm{NMR}\left(600 \mathrm{MHz}, \mathrm{CDCl}_{3}\right) \delta 8.09$ (s, 1H), 7.60 (d, $J=8.7 \mathrm{~Hz}$, $2 \mathrm{H}), 7.47(\mathrm{~d}, J=8.5 \mathrm{~Hz}, 2 \mathrm{H}), 7.43(\mathrm{~d}, J=8.7 \mathrm{~Hz}, 2 \mathrm{H}), 7.16(\mathrm{~d}, J=8.4 \mathrm{~Hz}, 2 \mathrm{H}), 4.34$ (dd, $J=$ 13.1, 7.1 Hz, 2H), 4.06-3.96 (m, 2H), 3.97-3.87 (m, 3H), 1.64-1.52 (m, 5H), 0.89-0.83 (m, $8 \mathrm{H}) ;{ }^{13} \mathrm{C} \mathrm{NMR}\left(151 \mathrm{MHz}, \mathrm{CDCl}_{3}\right) \delta 183.3(\mathrm{~d}, J=5.0 \mathrm{~Hz}), 137.6(\mathrm{~d}, J=2.4 \mathrm{~Hz}), 134.5(\mathrm{~d}, J=$ 
$12.9 \mathrm{~Hz}), 132.9,132.1,131.1,129.9,129.0,122.3(\mathrm{~d}, J=24.3 \mathrm{~Hz}), 69.1(\mathrm{~d}, J=7.3 \mathrm{~Hz}), 68.7$ $(\mathrm{d}, J=7.3 \mathrm{~Hz}), 57.8(\mathrm{~d}, J=151.6 \mathrm{~Hz}), 49.1(\mathrm{~d}, J=2.7 \mathrm{~Hz}), 23.8(\mathrm{~d}, J=5.6 \mathrm{~Hz}), 23.7(\mathrm{~d}, J=$ $6.0 \mathrm{~Hz}$ ), 9.9, 9.9; ${ }^{31} \mathrm{P}$ NMR (243 $\left.\mathrm{MHz}, \mathrm{CDCl}_{3}\right) \delta$ 19.18; HRMS (ESI): $\mathrm{m} / z$ calcd. for $\mathrm{C}_{22} \mathrm{H}_{25} \mathrm{Br}_{2} \mathrm{~N}_{2} \mathrm{NaO}_{4} \mathrm{P}[\mathrm{M}+\mathrm{Na}]^{+}:$592.9811, found: 592.9810 .

\section{Dipropyl ((3S, 4R)-4,6-bis(4-iodophenyl)-5-oxo-2,3,4,5-tetrahydropyridazin-3-yl)phosp honate (3ec)}

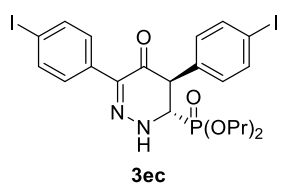

Isolated by silica gel column chromatography (petroleum ether : acetone $=$ $8: 1 \sim 1: 1, \mathrm{v} / \mathrm{v})$ to afford a yellow solid $(58.6 \mathrm{mg}, 88 \%$ yield, $90 \%$ ee, $>99: 1$ $d r), \operatorname{mp} 125.7-177.1{ }^{\circ} \mathrm{C} ;[\alpha]_{\mathrm{D}}^{20}=-168.2^{\circ}(c=1.0$, DCM $) ;$ HPLC (Chiralpak IA, $n$-hexane $/{ }^{i} \mathrm{PrOH}=80 / 20$, flow rate $=0.5 \mathrm{~mL} / \mathrm{min}, \mathrm{I}=254$ $\mathrm{nm}): t_{\mathrm{R}}=38.3 \min$ (major), $27.1 \mathrm{~min}$ (minor); ${ }^{1} \mathrm{H} \mathrm{NMR}\left(600 \mathrm{MHz}, \mathrm{CDCl}_{3}\right) \delta 7.69$ (t, $J=8.1$ $\mathrm{Hz}, 3 \mathrm{H}), 7.65(\mathrm{~d}, J=8.5 \mathrm{~Hz}, 2 \mathrm{H}), 7.48(\mathrm{~d}, J=8.3 \mathrm{~Hz}, 2 \mathrm{H}), 7.02(\mathrm{~d}, J=8.3 \mathrm{~Hz}, 2 \mathrm{H}), 4.31(\mathrm{dd}$, $J=12.9,8.0 \mathrm{~Hz}, 1 \mathrm{H}), 4.05-3.96(\mathrm{~m}, 2 \mathrm{H}), 3.96-3.86(\mathrm{~m}, 3 \mathrm{H}), 1.66-1.58(\mathrm{~m}, 2 \mathrm{H}), 1.58-1.52$ (m, 2H), 0.91-0.83 (m, 6H); ${ }^{13} \mathrm{C}$ NMR (151 MHz, $\left.\mathrm{CDCl}_{3}\right) \delta 183.4(\mathrm{~d}, J=6.0 \mathrm{~Hz}), 138.2$, $138.1,137.1,135.0$ (d, $J=11.8 \mathrm{~Hz}), 133.4,130.3,129.3,94.1,93.9,69.1$ (d, $J=7.2 \mathrm{~Hz}), 68.7$ $(\mathrm{d}, J=7.5 \mathrm{~Hz}), 57.9$ (d, $J=151.9 \mathrm{~Hz}), 49.5(\mathrm{~d}, J=2.4 \mathrm{~Hz}), 23.9(\mathrm{~d}, J=5.6 \mathrm{~Hz}), 23.7$ (d, $J=$ $5.7 \mathrm{~Hz}$ ), 10.0, 9.9; ${ }^{31} \mathrm{P}$ NMR (243 $\left.\mathrm{MHz}, \mathrm{CDCl}_{3}\right) \delta$ 18.97; HRMS (ESI): $\mathrm{m} / z$ calcd. for $\mathrm{C}_{22} \mathrm{H}_{25} \mathrm{I}_{2} \mathrm{~N}_{2} \mathrm{NaO}_{4} \mathrm{P}[\mathrm{M}+\mathrm{Na}]^{+}: 688.9534$, found: 688.9539 .

\section{Dipropyl ((3S, 4R)-5-oxo-4,6-bis(4-(trifluoromethyl)phenyl)-2,3,4,5-tetrahydro pyridazin-3-yl)phosphonate (3fc)}

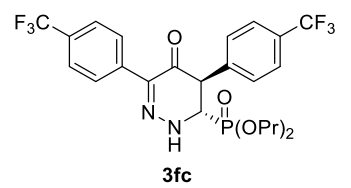

Isolated by silica gel column chromatography (petroleum ether : acetone $=8: 1 \sim 1: 1, \mathrm{v} / \mathrm{v})$ to afford a yellow oil $(47.3 \mathrm{mg}, 86 \%$ yield, $68 \%$ $e e,>99: 1 d r),[\alpha]_{\mathrm{D}}^{20}=-192.2^{\circ}(c=1.0, \mathrm{DCM})$; HPLC (Chiralpak IA, $n$-hexane $/{ }^{i} \mathrm{PrOH}=80 / 20$, flow rate $\left.=0.5 \mathrm{~mL} / \mathrm{min}, \mathrm{I}=254 \mathrm{~nm}\right): t_{\mathrm{R}}=$ $26.4 \mathrm{~min}$ (major), $18.7 \mathrm{~min}$ (minor); ${ }^{1} \mathrm{H} \mathrm{NMR}\left(600 \mathrm{MHz}, \mathrm{CDCl}_{3}\right) \delta 7.88(\mathrm{~d}, J=8.2 \mathrm{~Hz}, 2 \mathrm{H})$, 7.79 (s, 1H), $7.63(\mathrm{~d}, J=8.1 \mathrm{~Hz}, 2 \mathrm{H}), 7.58(\mathrm{~d}, J=8.2 \mathrm{~Hz}, 2 \mathrm{H}), 7.42(\mathrm{~d}, J=8.0 \mathrm{~Hz}, 2 \mathrm{H}), 4.40$ $(\mathrm{dd}, J=13.1,8.8 \mathrm{~Hz}, 1 \mathrm{H}), 4.15(\mathrm{dd}, J=11.3,8.7 \mathrm{~Hz}, 1 \mathrm{H}), 4.03(\mathrm{dq}, J=10.1,6.8 \mathrm{~Hz}, 1 \mathrm{H})$, $3.92(\mathrm{q}, J=6.9 \mathrm{~Hz}, 3 \mathrm{H}), 1.63(\mathrm{~h}, J=7.2 \mathrm{~Hz}, 2 \mathrm{H}), 1.53(\mathrm{~h}, J=7.2 \mathrm{~Hz}, 2 \mathrm{H}), 0.89$ (t, $J=7.4 \mathrm{~Hz}$, $3 \mathrm{H}), 0.83(\mathrm{t}, J=7.4 \mathrm{~Hz}, 3 \mathrm{H}) ;{ }^{13} \mathrm{C} \mathrm{NMR}\left(151 \mathrm{MHz}, \mathrm{CDCl}_{3}\right) \delta 183.1(\mathrm{~d}, J=6.6 \mathrm{~Hz}), 140.0$, $139.1(\mathrm{~d}, J=10.8 \mathrm{~Hz}), 137.8,137.1,130.6(\mathrm{q}, J=32.6 \mathrm{~Hz}), 130.1(\mathrm{~d}, J=32.4 \mathrm{~Hz}), 129.0$, 127.6, 125.9 (q, $J=3.9 \mathrm{~Hz}), 124.9(\mathrm{q}, J=3.8 \mathrm{~Hz}), 123.1(\mathrm{~d}, J=45.5 \mathrm{~Hz}), 69.2(\mathrm{~d}, J=7.2 \mathrm{~Hz})$, $68.8(\mathrm{~d}, J=7.3 \mathrm{~Hz}), 57.9(\mathrm{~d}, J=152.7 \mathrm{~Hz}), 49.8(\mathrm{~d}, J=3.1 \mathrm{~Hz}), 23.9(\mathrm{~d}, J=5.8 \mathrm{~Hz}), 23.7(\mathrm{~d}$, $J=6.0 \mathrm{~Hz}), 9.9,9.8 ;{ }^{19} \mathrm{~F}$ NMR $\left(565 \mathrm{MHz}, \mathrm{CDCl}_{3}\right) \delta-62.74,-62.92 ;{ }^{31} \mathrm{P}$ NMR $(243 \mathrm{MHz}$, 
$\left.\mathrm{CDCl}_{3}\right) \delta$ 18.54; HRMS (ESI): $m / z$ calcd. for $\mathrm{C}_{24} \mathrm{H}_{25} \mathrm{~F}_{6} \mathrm{~N}_{2} \mathrm{NaO}_{4} \mathrm{P}[\mathrm{M}+\mathrm{Na}]^{+}:$573.1348, found: 573.1348 .

\section{Dipropyl ((3S, 4R)-5-oxo-4,6-bis(4-(trifluoromethoxy)phenyl)-2,3,4,5-tetrahydropyrida zin-3-yl)phosphonate (3gc)}

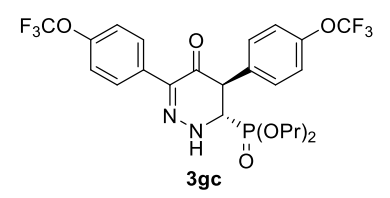

Isolated by silica gel column chromatography (petroleum ether : acetone $=8: 1 \sim 1: 1, \mathrm{v} / \mathrm{v})$ to afford a yellow oil $(55.2 \mathrm{mg}, 95 \%$ yield, $91 \%$ ee, $>99: 1 d r) ;[\alpha]_{\mathrm{D}}^{20}=-210.9^{\circ}(c=1.0$, DCM); HPLC (Chiralpak IA, $n$-hexane $/{ }^{i} \mathrm{PrOH}=80 / 20$, flow rate $=0.5 \mathrm{~mL} / \mathrm{min}, \mathrm{I}=254 \mathrm{~nm}$ ): $t_{\mathrm{R}}=17.0 \mathrm{~min}$ (major), $14.6 \mathrm{~min}$ (minor); ${ }^{1} \mathrm{H} \mathrm{NMR}\left(600 \mathrm{MHz}, \mathrm{CDCl}_{3}\right) \delta 7.88(\mathrm{dt}, J=9.4,4.7 \mathrm{~Hz}, 1 \mathrm{H}), 7.78(\mathrm{~d}, J=$ $8.9 \mathrm{~Hz}, 1 \mathrm{H}), 7.33(\mathrm{~d}, J=8.7 \mathrm{~Hz}, 1 \mathrm{H}), 7.21(\mathrm{~d}, J=8.3 \mathrm{~Hz}, 2 \mathrm{H}), 7.16(\mathrm{~d}, J=8.5 \mathrm{~Hz}, 2 \mathrm{H}), 4.08$ $(\mathrm{dd}, J=11.3,8.1 \mathrm{~Hz}, 1 \mathrm{H}), 4.07-3.98(\mathrm{~m}, 1 \mathrm{H}), 3.97-3.87(\mathrm{~m}, 3 \mathrm{H}), 1.63(\mathrm{~h}, J=7.2 \mathrm{~Hz}, 2 \mathrm{H})$, $1.56(\mathrm{~h}, J=7.4 \mathrm{~Hz}, 2 \mathrm{H}), 0.91-0.83(\mathrm{~m}, 6 \mathrm{H}) ;{ }^{13} \mathrm{C} \mathrm{NMR}\left(151 \mathrm{MHz}, \mathrm{CDCl}_{3}\right) \delta 183.5(\mathrm{~d}, J=5.8$ $\mathrm{Hz}), 149.0(\mathrm{~d}, J=13.9 \mathrm{~Hz}), 137.8,134.1$ (d, $J=11.5 \mathrm{~Hz}), 132.5,130.0,129.0,121.4,120.5$ (q, $J=257.2 \mathrm{~Hz}), 120.4(\mathrm{q}, J=257.5 \mathrm{~Hz}), 120.3,69.1(\mathrm{~d}, J=7.3 \mathrm{~Hz}), 68.7(\mathrm{~d}, J=7.5 \mathrm{~Hz}), 58.0$ $(\mathrm{d}, J=152.2 \mathrm{~Hz}), 49.3(\mathrm{~d}, J=2.9 \mathrm{~Hz}), 23.9(\mathrm{~d}, J=5.7 \mathrm{~Hz}), 23.7(\mathrm{~d}, J=5.9 \mathrm{~Hz}), 9.9,9.8 ;{ }^{19} \mathrm{~F}$ NMR $\left(565 \mathrm{MHz}, \mathrm{CDCl}_{3}\right) \delta-57.79,-57.89 ;{ }^{31} \mathrm{P}$ NMR $\left(243 \mathrm{MHz}, \mathrm{CDCl}_{3}\right) \delta 19.00 ; \mathrm{HRMS}$ (ESI) : $m / z$ calcd. for $\mathrm{C}_{24} \mathrm{H}_{25} \mathrm{~F}_{6} \mathrm{~N}_{2} \mathrm{NaO}_{6} \mathrm{P}[\mathrm{M}+\mathrm{Na}]^{+}$: 605.1247, found: 605.1242 .

\section{Dipropyl ((3S, 4R)-4,6-bis(3-fluorophenyl)-5-oxo-2,3,4,5-tetrahydropyridazin-3-yl)phos -phonate (3hc)}

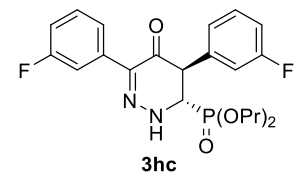

Isolated by silica gel column chromatography (petroleum ether : acetone $=$ $8: 1 \sim 1: 1, \mathrm{v} / \mathrm{v})$ to afford a yellow oil $(41.8 \mathrm{mg}, 93 \%$ yield, $81 \%$ ee, $>99: 1$

$d r) ;[\alpha]_{\mathrm{D}}^{20}=-446.6^{\circ}(c=1.0$, DCM $) ;$ HPLC (Chiralpak IC, $n$-hexane $/{ }^{i} \mathrm{PrOH}=80 / 20$, flow rate $=0.5 \mathrm{~mL} / \mathrm{min}, \mathrm{I}=254 \mathrm{~nm}$ ): $t_{\mathrm{R}}=13.7 \mathrm{~min}$ (major), 15.0 $\min$ (minor); ${ }^{1} \mathrm{H} \mathrm{NMR}\left(600 \mathrm{MHz}, \mathrm{CDCl}_{3}\right) \delta 8.05(\mathrm{~s}, 1 \mathrm{H}), 7.55(\mathrm{~d}, J=7.9 \mathrm{~Hz}, 1 \mathrm{H}), 7.49$ (d, $J=$ $10.5 \mathrm{~Hz}, 1 \mathrm{H}), 7.35-7.28(\mathrm{~m}, 1 \mathrm{H}), 7.30-7.23(\mathrm{~m}, 1 \mathrm{H}), 7.08$ (d, J= 7.8 Hz, 1H), 7.05-6.93 (m, $3 \mathrm{H}), 4.38(\mathrm{dd}, J=13.1,6.5 \mathrm{~Hz}, 1 \mathrm{H}), 4.08-3.99$ (m, 2H), 4.01-3.89 (m, 3H), 1.66-1.54 (m, $4 \mathrm{H}), 0.87(\mathrm{t}, J=7.4 \mathrm{~Hz}, 6 \mathrm{H}) ;{ }^{13} \mathrm{C} \mathrm{NMR}\left(150 \mathrm{MHz}, \mathrm{CDCl}_{3}\right) \delta 183.0(\mathrm{~d}, J=4.6 \mathrm{~Hz}), 163.0$ (d, $J$ $=247.1 \mathrm{~Hz}), 162.6(\mathrm{~d}, J=244.2 \mathrm{~Hz}), 137.9(\mathrm{dd}, J=13.3,7.2 \mathrm{~Hz}), 137.3,136.1(\mathrm{~d}, J=8.1$ $\mathrm{Hz}), 130.5$ (d, $J=8.2 \mathrm{~Hz}), 129.3(\mathrm{~d}, J=8.2 \mathrm{~Hz}), 123.9$ (d, $J=2.9 \mathrm{~Hz}), 123.0$ (d, $J=2.8 \mathrm{~Hz})$, $115.3(\mathrm{~d}, J=22.4 \mathrm{~Hz}), 115.2$ (d, $J=20.9 \mathrm{~Hz}), 114.7$ (d, $J=21.2 \mathrm{~Hz}), 114.3(\mathrm{~d}, J=23.5 \mathrm{~Hz})$, $69.1(\mathrm{~d}, J=7.4 \mathrm{~Hz}), 68.7(\mathrm{~d}, J=7.6 \mathrm{~Hz}), 57.8(\mathrm{~d}, J=151.3 \mathrm{~Hz}), 49.2,23.8(\mathrm{~d}, J=5.8 \mathrm{~Hz})$, $23.7(\mathrm{~d}, J=5.9 \mathrm{~Hz}), 9.9,9.9 ;{ }^{19} \mathrm{~F}$ NMR $\left(565 \mathrm{MHz}, \mathrm{CDCl}_{3}\right) \delta-112.40,-114.17 ;{ }^{31} \mathrm{P}$ NMR $(243$ $\mathrm{MHz}, \mathrm{CDCl}_{3}$ ) $\delta$ 19.22; HRMS (ESI): $m / z$ calcd. for $\mathrm{C}_{22} \mathrm{H}_{25} \mathrm{~F}_{2} \mathrm{~N}_{2} \mathrm{NaO}_{4} \mathrm{P}[\mathrm{M}+\mathrm{Na}]^{+}:$473.1412, 
found: 473.1412 .

Dipropyl ((3S, 4R)-4,6-bis(3-chlorophenyl)-5-oxo-2,3,4,5-tetrahydropyridazin-3-yl)phos -phonate (3ic)

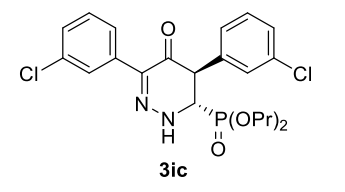

Isolated by silica gel column chromatography (petroleum ether : acetone $=8: 1 \sim 1: 1, \mathrm{v} / \mathrm{v})$ to afford a yellow solid $(45.4 \mathrm{mg}, 94 \%$ yield, $69 \%$ $e e,>99: 1 d r), \operatorname{mp} 133.1-134.6^{\circ} \mathrm{C} ;[\alpha]_{\mathrm{D}}^{20}=-50.6^{\circ}(c=1.0, \mathrm{DCM}) ; \mathrm{HPLC}$

(Chiralpak IC, $n$-hexane $/{ }^{i} \mathrm{PrOH}=80 / 20$, flow rate $=0.5 \mathrm{~mL} / \mathrm{min}, \mathrm{I}=254 \mathrm{~nm}$ ): $t_{\mathrm{R}}=14.8 \mathrm{~min}$ (major), $15.9 \mathrm{~min}$ (minor); ${ }^{1} \mathrm{H} \mathrm{NMR}\left(600 \mathrm{MHz}, \mathrm{CDCl}_{3}\right) \delta 8.02(\mathrm{~s}, 1 \mathrm{H}), 7.76$ (d, $J=2.2 \mathrm{~Hz}$, $1 \mathrm{H}), 7.65(\mathrm{dt}, J=5.9,1.8 \mathrm{~Hz}, 1 \mathrm{H}), 7.31-7.28(\mathrm{~m}, 3 \mathrm{H}), 7.27-7.23(\mathrm{~m}, 2 \mathrm{H}), 7.18(\mathrm{~d}, J=6.4 \mathrm{~Hz}$, $1 \mathrm{H}), 4.37$ (dd, $J=13.3,7.4 \mathrm{~Hz}, 1 \mathrm{H}), 4.06-4.00(\mathrm{~m}, 2 \mathrm{H}), 3.98-3.91(\mathrm{~m}, 3 \mathrm{H}), 1.66-1.54(\mathrm{~m}$, 4H), 0.91-0.85 (m, 6H); $\left.{ }^{13} \mathrm{C} \mathrm{NMR} \mathrm{(150} \mathrm{MHz,} \mathrm{CDCl}_{3}\right) \delta 183.0(\mathrm{~d}, J=5.0 \mathrm{~Hz}), 137.4,137.4$ (d), $137.3(\mathrm{~d}, J=6.1 \mathrm{~Hz}), 135.6,134.9,134.0,130.2,129.2,128.5,128.4,128.0,127.4,126.5$, 125.6, $69.1(\mathrm{~d}, J=7.4 \mathrm{~Hz}), 68.8(\mathrm{~d}, J=7.5 \mathrm{~Hz}), 57.8(\mathrm{~d}, J=151.9 \mathrm{~Hz}), 49.3(\mathrm{~d}, J=2.6 \mathrm{~Hz})$, $23.9(\mathrm{~d}, J=5.8 \mathrm{~Hz}), 23.7(\mathrm{~d}, J=5.9 \mathrm{~Hz}), 9.9,9.9 ;{ }^{31} \mathrm{P}$ NMR $\left(243 \mathrm{MHz}, \mathrm{CDCl}_{3}\right) \delta 19.02$; HRMS (ESI): $m / z$ calcd. for $\mathrm{C}_{22} \mathrm{H}_{25} \mathrm{C}_{12} \mathrm{~N}_{2} \mathrm{NaO}_{4} \mathrm{P}[\mathrm{M}+\mathrm{Na}]^{+}: 505.0821$, found: 505.0821.

\section{Dipropyl ((3S, 4R)-4-(6-fluorocyclohexa-1,5-dien-1-yl)-6-(2-fluorophenyl)-5-oxo-2,3,4,5-} tetrahydropyridazin-3-yl)phosphonate $(3 \mathrm{jc})$

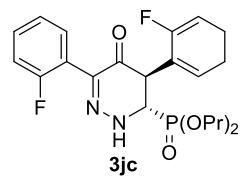

Isolated by silica gel column chromatography (petroleum ether : acetone $=$ $8: 1 \sim 1: 1, \mathrm{v} / \mathrm{v})$ to afford a colorless oil $(25.8 \mathrm{mg}, 57 \%$ yield, $89 \%$ ee, $>99: 1$ $d r) ;[\alpha]_{\mathrm{D}}^{20}=-199.1^{\circ}(c=1.0, \mathrm{DCM}) ; \mathrm{HPLC}\left(\right.$ Chiralpak IC, $n$-hexane $/{ }^{i} \mathrm{PrOH}=$ 70/30, flow rate $=0.5 \mathrm{~mL} / \mathrm{min}, \mathrm{I}=254 \mathrm{~nm}$ ): $t_{\mathrm{R}}=32.4 \mathrm{~min}$ (major), $28.5 \min$ (minor); ${ }^{1} \mathrm{H}$ NMR (600 MHz, $\left.\mathrm{CDCl}_{3}\right) \delta 7.49(\mathrm{~s}, 1 \mathrm{H}), 7.37(\mathrm{td}, J=7.4,1.8 \mathrm{~Hz}, 1 \mathrm{H}), 7.36-7.28(\mathrm{~m}, 2 \mathrm{H})$, 7.30-7.24 (m, 1H), 7.18-7.10 (m, 2H), 7.11-7.04 (m, 2H), $4.50(\mathrm{t}, J=12.3 \mathrm{~Hz}, 1 \mathrm{H}), 4.27$ (dd, $J=12.5,10.7 \mathrm{~Hz}, 1 \mathrm{H}), 4.05-3.97(\mathrm{~m}, 1 \mathrm{H}), 3.96-3.87(\mathrm{~m}, 1 \mathrm{H}), 3.89-3.80(\mathrm{~m}, 2 \mathrm{H}), 1.65(\mathrm{~h}, J$ $=7.2 \mathrm{~Hz}, 2 \mathrm{H}), 1.48(\mathrm{~h}, J=7.1 \mathrm{~Hz}, 2 \mathrm{H}), 0.92(\mathrm{t}, J=7.4 \mathrm{~Hz}, 3 \mathrm{H}), 0.81(\mathrm{t}, J=7.4 \mathrm{~Hz}, 3 \mathrm{H}) ;{ }^{13} \mathrm{C}$ NMR $\left(151 \mathrm{MHz}, \mathrm{CDCl}_{3}\right) \delta 182.3(\mathrm{~d}, J=10.8 \mathrm{~Hz}), 160.8(\mathrm{~d}, J=247.1 \mathrm{~Hz}), 160.6(\mathrm{~d}, J=$ $249.5 \mathrm{~Hz}$ ), 139.1, 131.5 (d, $J=4.3 \mathrm{~Hz}), 130.9$ (d, $J=3.4 \mathrm{~Hz}), 130.3$ (d, $J=8.3 \mathrm{~Hz}), 129.9$ (d, $J=8.4 \mathrm{~Hz}), 124.3(\mathrm{~d}, J=3.4 \mathrm{~Hz}), 123.8(\mathrm{~d}, J=3.7 \mathrm{~Hz}), 122.2(\mathrm{dd}, J=14.5,5.4 \mathrm{~Hz}), 122.0(\mathrm{~d}$, $J=14.7 \mathrm{~Hz}), 115.8,115.6,69.0(\mathrm{~d}, J=7.1 \mathrm{~Hz}), 68.4(\mathrm{~d}, J=7.1 \mathrm{~Hz}), 56.9(\mathrm{dd}, J=154.5,2.4$ $\mathrm{Hz}), 47.0(\mathrm{~d}, J=4.8 \mathrm{~Hz}), 23.9(\mathrm{~d}, J=5.8 \mathrm{~Hz}), 23.6(\mathrm{~d}, J=6.2 \mathrm{~Hz}), 9.9,9.8 ;{ }^{19} \mathrm{~F}$ NMR $(565$ $\left.\mathrm{MHz}, \mathrm{CDCl}_{3}\right) \delta-112.12,-112.61 ;{ }^{31} \mathrm{P}$ NMR (243 MHz, $\left.\mathrm{CDCl}_{3}\right) \delta 18.37$; HRMS (ESI): $\mathrm{m} / \mathrm{z}$ calcd. for $\mathrm{C}_{22} \mathrm{H}_{25} \mathrm{~F}_{2} \mathrm{~N}_{2} \mathrm{NaO}_{4} \mathrm{P}[\mathrm{M}+\mathrm{Na}]^{+}$: 473.1412, found: 473.1411 . 
Dipropyl ((3S, 4R)-4,6-bis(2-chlorophenyl)-5-oxo-2,3,4,5-tetrahydropyridazin-3-yl)phos -phonate $(3 \mathrm{kc})$

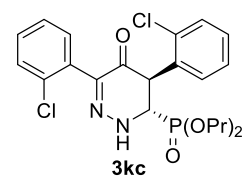

Isolated by silica gel column chromatography (petroleum ether : acetone $=$ $8: 1 \sim 1: 1, \mathrm{v} / \mathrm{v})$ to afford a yellow solid $(25.1 \mathrm{mg}, 52 \%$ yield, $90 \%$ ee, $>99: 1$ $d r), \operatorname{mp} 37.0-38.1^{\circ} \mathrm{C} ;[\alpha]_{\mathrm{D}}^{20}=-125.2^{\circ}(c=1.0, \mathrm{DCM})$; HPLC (Chiralpak ASH, $n$-hexane $/{ }^{i} \mathrm{PrOH}=70 / 30$, flow rate $=0.5 \mathrm{~mL} / \mathrm{min}, \mathrm{I}=254 \mathrm{~nm}$ ) $: t_{\mathrm{R}}=17.2 \mathrm{~min}$ (major), 12.2 min (minor); ${ }^{1} \mathrm{H}$ NMR (600 MHz, $\left.\mathrm{CDCl}_{3}\right) \delta 7.54$ (s, 1H), 7.43-7.38 (m, 2H), 7.35-7.32 (m, 2H), 7.31-7.22 (m, 4H), $4.66(\mathrm{t}, J=11.8 \mathrm{~Hz}, 1 \mathrm{H}), 4.53$ (t, $J=11.2 \mathrm{~Hz}, 1 \mathrm{H}), 4.07-4.00$ (m, $1 \mathrm{H}), 3.98-3.91(\mathrm{~m}, 1 \mathrm{H}), 3.88(\mathrm{q}, J=6.8 \mathrm{~Hz}, 2 \mathrm{H}), 1.67(\mathrm{~h}, J=7.2 \mathrm{~Hz}, 2 \mathrm{H}), 1.51(\mathrm{~h}, J=6.9 \mathrm{~Hz}$, $2 \mathrm{H}), 0.92(\mathrm{t}, J=7.4 \mathrm{~Hz}, 3 \mathrm{H}), 0.82(\mathrm{t}, J=7.4 \mathrm{~Hz}, 3 \mathrm{H}) ;{ }^{13} \mathrm{C} \mathrm{NMR}\left(151 \mathrm{MHz}, \mathrm{CDCl}_{3}\right) \delta 182.2$ (d, $J=9.4 \mathrm{~Hz}), 141.8,134.1,134.0,133.0$ (d, $J=6.6 \mathrm{~Hz}), 133.0,131.5,131.3,130.1,129.7$, 129.6, 129.3, 127.1, 126.5, 69.0 (d, $J=7.3 \mathrm{~Hz}), 68.5$ (d, $J=7.3 \mathrm{~Hz}), 56.7$ (d, $J=153.5 \mathrm{~Hz})$, 49.0, $23.9(\mathrm{~d}, J=5.7 \mathrm{~Hz}), 23.6$ (d, $J=6.1 \mathrm{~Hz}), 9.9,9.9 ;{ }^{31} \mathrm{P}$ NMR $\left(243 \mathrm{MHz}, \mathrm{CDCl}_{3}\right) \delta 18.72$; HRMS (ESI): $m / z$ calcd. for $\mathrm{C}_{22} \mathrm{H}_{25} \mathrm{C}_{12} \mathrm{~N}_{2} \mathrm{NaO}_{4} \mathrm{P}[\mathrm{M}+\mathrm{Na}]^{+}: 505.0821$, found: 505.0821 .

Dipropyl ((3S, 4R)-4,6-bis(2-bromophenyl)-5-oxo-2,3,4,5-tetrahydropyridazin-3-yl)phos -phonate (3lc)

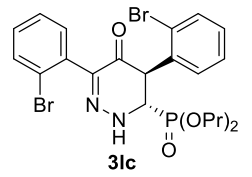

Isolated by silica gel column chromatography (petroleum ether : acetone $=$ $8: 1 \sim 1: 1, \mathrm{v} / \mathrm{v})$ to afford a yellow solid $(31.5 \mathrm{mg}, 55 \%$ yield, $86 \%$ ee, $>99: 1$ $d r), \operatorname{mp} 43.1-44.7^{\circ} \mathrm{C} ;[\alpha]_{\mathrm{D}}^{20}=-132.4^{\circ}(c=1.0, \mathrm{DCM})$; HPLC (Chiralpak IC, $n$-hexane $/ \mathrm{PrOH}=70 / 30$, flow rate $=0.5 \mathrm{~mL} / \mathrm{min}, \mathrm{I}=254 \mathrm{~nm}$ ): $t_{\mathrm{R}}=45.1 \mathrm{~min}$ (major), 39.8 min (minor); ${ }^{1} \mathrm{H}$ NMR (600 MHz, $\left.\mathrm{CDCl}_{3}\right) \delta$ 7.62-7.57 (m, 2H), 7.49 (s, 1H), 7.36-7.28 (m, 4H), 7.23-7.13 (m, 2H), 4.71-4.56 (m, 2H), 4.09-4.01 (m, 1H), 4.00-3.91 (m, 1H), 3.92-3.79 $(\mathrm{m}, 2 \mathrm{H}), 1.68(\mathrm{~h}, J=7.1 \mathrm{~Hz}, 2 \mathrm{H}), 1.54-1.46(\mathrm{~m}, 2 \mathrm{H}), 0.93(\mathrm{t}, J=7.4 \mathrm{~Hz}, 3 \mathrm{H}), 0.82$ (t, $J=7.4$ $\mathrm{Hz}, 3 \mathrm{H}) ;{ }^{13} \mathrm{C}$ NMR $\left(151 \mathrm{MHz}, \mathrm{CDCl}_{3}\right) \delta 182.2(\mathrm{~d}, J=9.6 \mathrm{~Hz}), 143.1,134.8,134.7$ (d, $J=6.7$ Hz), 133.4, 132.8, 131.6, 131.1, 129.9, 129.5, 127.6, 127.1, 124.8, 123.8, 69.1 (d, $J=7.2 \mathrm{~Hz})$, $68.5(\mathrm{~d}, J=7.2 \mathrm{~Hz}), 57.0(\mathrm{~d}, J=153.6 \mathrm{~Hz}), 50.8,23.9(\mathrm{~d}, J=5.5 \mathrm{~Hz}), 23.7(\mathrm{~d}, J=6.1 \mathrm{~Hz})$, 10.0, 9.9; ${ }^{31} \mathrm{P}$ NMR (243 $\left.\mathrm{MHz}, \mathrm{CDCl}_{3}\right) \delta$ 18.65; HRMS (ESI): $\mathrm{m} / \mathrm{z}$ calcd. for $\mathrm{C}_{22} \mathrm{H}_{25} \mathrm{Br}_{2} \mathrm{~N}_{2} \mathrm{NaO}_{4} \mathrm{P}[\mathrm{M}+\mathrm{Na}]^{+}:$592.9811, found: 592.9809 .

Dipropyl ((3S, 4R)-5-oxo-4,6-bis(2-(trifluoromethyl)phenyl)-2,3,4,5-tetrahydropyridazi n-3-yl)phosphonate $(3 \mathrm{mc})$

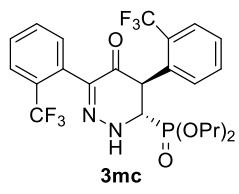

Isolated by silica gel column chromatography (petroleum ether : acetone $=$ 8:1 1:1, v/v) to afford a white solid $(30.2 \mathrm{mg}, 55 \%$ yield, $86 \%$ ee, $>99: 1 \mathrm{dr})$, mp 139.7-141.1 ${ }^{\circ} \mathrm{C} ;[\alpha]_{\mathrm{D}}^{20}=-140.0^{\circ}(c=1.0, \mathrm{DCM})$; HPLC (Chiralpak IC, 
$n$-hexane $/{ }^{i} \mathrm{PrOH}=80 / 20$, flow rate $=0.5 \mathrm{~mL} / \mathrm{min}, \mathrm{I}=254 \mathrm{~nm}$ ): $t_{\mathrm{R}}=13.1 \mathrm{~min}$ (major), 14.3 $\min$ (minor); ${ }^{1} \mathrm{H}$ NMR (600 MHz, $\left.\mathrm{CDCl}_{3}\right) \delta 7.70(\mathrm{dd}, J=16.0,7.8 \mathrm{~Hz}, 2 \mathrm{H}), 7.58-7.50(\mathrm{~m}$, 2H), 7.49-7.38 (m, 4H), $7.37(\mathrm{~d}, J=7.7 \mathrm{~Hz}, 1 \mathrm{H}), 4.55(\mathrm{t}, J=10.7 \mathrm{~Hz}, 1 \mathrm{H}), 4.48(\mathrm{t}, J=11.4$ $\mathrm{Hz}, 1 \mathrm{H}), 4.13-4.05(\mathrm{~m}, 1 \mathrm{H}), 4.07-3.97(\mathrm{~m}, 1 \mathrm{H}), 3.88-3.82(\mathrm{~m}, 2 \mathrm{H}), 1.70(\mathrm{~h}, J=7.3 \mathrm{~Hz}, 3 \mathrm{H})$, $1.52-1.44(\mathrm{~m}, 2 \mathrm{H}), 0.93(\mathrm{t}, J=7.4 \mathrm{~Hz}, 3 \mathrm{H}), 0.80(\mathrm{t}, J=7.4 \mathrm{~Hz}, 3 \mathrm{H}) ;{ }^{13} \mathrm{C}$ NMR $(150 \mathrm{MHz}$, $\left.\mathrm{CDCl}_{3}\right) \delta 182.8(\mathrm{~d}, J=8.9 \mathrm{~Hz}), 141.9,134.3-133.2(\mathrm{~m}), 132.4-132.2(\mathrm{~m}), 132.0,132.0(\mathrm{~d}, J=$ $9.3 \mathrm{~Hz}), 131.3,129.6$ (d, $J=21.6 \mathrm{~Hz}), 129.4(\mathrm{~d}, J=22.4 \mathrm{~Hz}), 129.2-128.5(\mathrm{~m}), 127.9,126.5$ $(\mathrm{d}, J=4.9 \mathrm{~Hz}), 125.0(\mathrm{~d}, J=9.8 \mathrm{~Hz}), 123.2(\mathrm{~d}, J=10.0 \mathrm{~Hz}), 69.3(\mathrm{~d}, J=7.1 \mathrm{~Hz}), 68.5(\mathrm{~d}, J=$ $7.4 \mathrm{~Hz}), 57.5(\mathrm{~d}, J=153.2 \mathrm{~Hz}), 46.6,23.8(\mathrm{~d}, J=5.2 \mathrm{~Hz}), 23.6(\mathrm{~d}, J=6.0 \mathrm{~Hz}), 9.8,9.8 ;{ }^{19} \mathrm{~F}$ NMR $\left(565 \mathrm{MHz}, \mathrm{CDCl}_{3}\right) \delta-57.98,-58.18 ;{ }^{31} \mathrm{P} \mathrm{NMR}\left(243 \mathrm{MHz}, \mathrm{CDCl}_{3}\right) \delta 18.34 ; \mathrm{HRMS}$ (ESI): $m / z$ calcd. for $\mathrm{C}_{24} \mathrm{H}_{25} \mathrm{~F}_{6} \mathrm{~N}_{2} \mathrm{NaO}_{4} \mathrm{P}[\mathrm{M}+\mathrm{Na}]^{+}: 573.1348$, found: 573.1351 .

Dipropyl $\quad((3 S, \quad 4 R)-5-0 x o-4,6-d i-p$-tolyl-2,3,4,5-tetrahydropyridazin-3-yl)phosphonate (3nc)

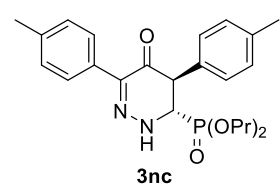
Isolated by silica gel column chromatography (petroleum ether : acetone $=$ $8: 1 \sim 1: 1, \mathrm{v} / \mathrm{v})$ to afford a yellow oil $(14.1 \mathrm{mg}, 32 \%$ yield, $73 \%$ ee, $>99: 1$ $d r),[\alpha]_{\mathrm{D}}^{20}=-124.4^{\circ}(c=1.0, \mathrm{DCM})$; HPLC (Chiralpak IA, $n$-hexane $/^{i} \mathrm{PrOH}$ $=80 / 20$, flow rate $=0.5 \mathrm{~mL} / \mathrm{min}, \mathrm{I}=254 \mathrm{~nm}$ ): $t_{\mathrm{R}}=26.5 \min$ (major), $23.5 \min ($ minor $) ;{ }^{1} \mathrm{H}$ NMR $\left(600 \mathrm{MHz}, \mathrm{CDCl}_{3}\right) \delta 7.59(\mathrm{~d}, J=7.9 \mathrm{~Hz}, 2 \mathrm{H}), 7.54(\mathrm{~s}, 1 \mathrm{H}), 7.19(\mathrm{~d}, J=7.8 \mathrm{~Hz}, 2 \mathrm{H})$, 7.15-7.11 (m, 4H), $4.34(\mathrm{dd}, J=12.6,6.9 \mathrm{~Hz}, 1 \mathrm{H}), 4.04-3.98(\mathrm{~m}, 2 \mathrm{H}), 3.94(\mathrm{q}, J=6.8 \mathrm{~Hz}$, 2H), 3.91-3.86 (m, 1H), 2.33 (s, 3H), $2.32(\mathrm{~s}, 3 \mathrm{H}), 1.63-1.54(\mathrm{~m}, 4 \mathrm{H}), 0.89-0.84(\mathrm{~m}, 6 \mathrm{H}) ;{ }^{13} \mathrm{C}$ NMR $\left(151 \mathrm{MHz}, \mathrm{CDCl}_{3}\right) \delta 184.4(\mathrm{~d}, J=4.8 \mathrm{~Hz}), 139.5,137.8(\mathrm{~d}, J=8.4 \mathrm{~Hz}), 132.8(\mathrm{~d}, J=$ 13.1 Hz), 131.3, 129.6, 128.6, 128.1, 127.6, 68.8 (d, $J=7.2 \mathrm{~Hz}), 68.5$ (d, J = 7.5 Hz), $58.2(\mathrm{~d}$, $J=150.4 \mathrm{~Hz}), 49.4,23.9(\mathrm{~d}, J=5.8 \mathrm{~Hz}), 23.7(\mathrm{~d}, J=5.9 \mathrm{~Hz}), 21.2,21.0,9.9,9.9 ;{ }^{31} \mathrm{P} \mathrm{NMR}$ (243 MHz, $\mathrm{CDCl}_{3}$ ) $\delta$ 19.94; HRMS (ESI): $\mathrm{m} / z$ calcd. for $\mathrm{C}_{24} \mathrm{H}_{31} \mathrm{~N}_{2} \mathrm{NaO}_{4} \mathrm{P}[\mathrm{M}+\mathrm{Na}]^{+}: 465.1914$, found: 465.1916 .

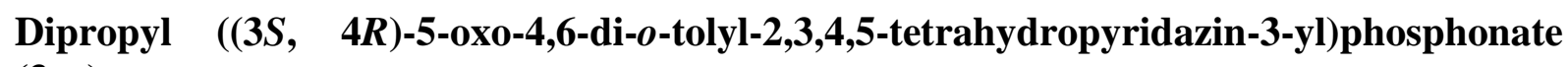
(3oc)

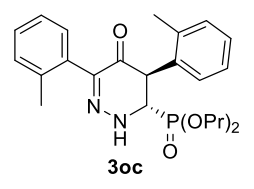

Isolated by silica gel column chromatography (petroleum ether : acetone $=$ $8: 1 \sim 1: 1, \mathrm{v} / \mathrm{v})$ to afford a colorless oil $(29.6 \mathrm{mg}, 67 \%$ yield, $94 \%$ ee, $>99: 1$ $d r),[\alpha]_{\mathrm{D}}^{20}=-144.9^{\circ}(c=1.0, \mathrm{DCM})$; HPLC (Chiralpak IA, $n$-hexane $/^{i} \mathrm{PrOH}=$ $80 / 20$, flow rate $=0.5 \mathrm{~mL} / \mathrm{min}, \mathrm{I}=254 \mathrm{~nm}$ ): $t_{\mathrm{R}}=20.5 \mathrm{~min}$ (major), $16.8 \mathrm{~min}$ (minor); ${ }^{1} \mathrm{H}$ NMR (600 MHz, $\left.\mathrm{CDCl}_{3}\right) \delta 7.30$ (brs, 1H), 7.25-7.15 (m, 8H), 4.38 (d, J=6.2 Hz, 2H), 4.02$3.90(\mathrm{~m}, 3 \mathrm{H}), 3.82-3.73(\mathrm{~m}, 1 \mathrm{H}), 2.44(\mathrm{~s}, 3 \mathrm{H}), 2.18(\mathrm{~s}, 3 \mathrm{H}), 1.68-1.55(\mathrm{~m}, 4 \mathrm{H}), 0.93-0.85$ (m, 
$6 \mathrm{H}) ;{ }^{13} \mathrm{C} \mathrm{NMR}\left(151 \mathrm{MHz}, \mathrm{CDCl}_{3}\right) \delta 184.0(\mathrm{~d}, J=7.1 \mathrm{~Hz}), 143.0,137.2,136.9,134.8(\mathrm{~d}, J=$ $10.2 \mathrm{~Hz}), 133.5,130.9,130.3,129.9,128.4,128.0,127.7,126.6,125.4,68.8$ (d, $J=7.2 \mathrm{~Hz})$, $68.5(\mathrm{~d}, J=7.4 \mathrm{~Hz}), 58.0(\mathrm{~d}, J=152.4 \mathrm{~Hz}), 46.4,23.9(\mathrm{~d}, J=5.6 \mathrm{~Hz}), 23.8(\mathrm{~d}, J=5.7 \mathrm{~Hz})$, 20.1, 20.0, 9.9; ${ }^{31} \mathrm{P}$ NMR (243 MHz, $\left.\mathrm{CDCl}_{3}\right) \delta$ 19.81; HRMS (ESI): $\mathrm{m} / z$ calcd. for $\mathrm{C}_{24} \mathrm{H}_{31} \mathrm{~N}_{2} \mathrm{NaO}_{4} \mathrm{P}[\mathrm{M}+\mathrm{Na}]^{+}$: 465.1914 , found: 465.1904 .

\section{Dipropyl ((3S, 4R)-4,6-bis(2,4-difluorophenyl)-5-oxo-2,3,4,5-tetrahydropyridazin-3-yl) phosphonate (3pc)}

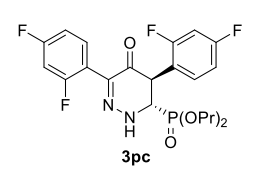

Isolated by silica gel column chromatography (petroleum ether : acetone $=$ $8: 1 \sim 1: 1, \mathrm{v} / \mathrm{v})$ to afford a colorless oil $(37.9 \mathrm{mg}, 78 \%$ yield, $88 \%$ ee, $>99: 1$ $d r),[\alpha]_{\mathrm{D}}^{20}=-190.6^{\circ}(c=1.0, \mathrm{DCM})$; HPLC (Chiralpak IA, $n$-hexane ${ }^{i} \mathrm{PrOH}=$ $80 / 20$, flow rate $=0.5 \mathrm{~mL} / \mathrm{min}, \mathrm{I}=254 \mathrm{~nm}$ ): $t_{\mathrm{R}}=29.4 \mathrm{~min}$ (major), $32.4 \mathrm{~min}$ (minor); ${ }^{1} \mathrm{H}$ NMR (600 MHz, $\left.\mathrm{CDCl}_{3}\right) \delta 7.61$ (brs, $\left.1 \mathrm{H}\right), 7.33(\mathrm{td}, J=8.3,6.3 \mathrm{~Hz}, 1 \mathrm{H}), 7.21(\mathrm{td}, J=8.3,6.1$ $\mathrm{Hz}, 1 \mathrm{H}), 6.92-6.76(\mathrm{~m}, 4 \mathrm{H}), 4.44(\mathrm{t}, J=12.5 \mathrm{~Hz}, 1 \mathrm{H}), 4.21(\mathrm{dd}, J=13.0,10.6 \mathrm{~Hz}, 1 \mathrm{H}), 4.03$ (dq, $J=10.0,7.2 \mathrm{~Hz}, 1 \mathrm{H}), 4.00-3.91(\mathrm{~m}, 1 \mathrm{H}), 3.87$ (q, $J=6.9 \mathrm{~Hz}, 2 \mathrm{H}), 1.66(\mathrm{~h}, J=7.1 \mathrm{~Hz}$, 2H), $1.50(\mathrm{~h}, J=7.1 \mathrm{~Hz}, 2 \mathrm{H}), 0.93(\mathrm{t}, J=7.4 \mathrm{~Hz}, 3 \mathrm{H}), 0.82(\mathrm{t}, J=7.4 \mathrm{~Hz}, 3 \mathrm{H}) ;{ }^{13} \mathrm{C} \mathrm{NMR}(151$ $\left.\mathrm{MHz}, \mathrm{CDCl}_{3}\right) \delta 182.0(\mathrm{~d}, J=10.9 \mathrm{~Hz}), 163.9(\mathrm{dd}, J=40.5,11.9 \mathrm{~Hz}), 162.2(\mathrm{dd}, J=40.0,11.9$ $\mathrm{Hz}), 161.7$ (dd, $J=19.4,12.6 \mathrm{~Hz}), 160.0(\mathrm{dd}, J=20.4,12.8 \mathrm{~Hz}), 138.1$ (d, $J=7.4 \mathrm{~Hz}), 132.2$ $(\mathrm{dd}, J=9.7,6.0 \mathrm{~Hz}), 131.8(\mathrm{dd}, J=9.6,4.9 \mathrm{~Hz}), 118.1(\mathrm{td}, J=15.1,9.0 \mathrm{~Hz}), 118.1(\mathrm{t}, \mathrm{J}=$ $14.9 \mathrm{~Hz}), 111.5(\mathrm{dd}, J=21.3,3.7 \mathrm{~Hz}), 111.1(\mathrm{dd}, J=21.2,3.7 \mathrm{~Hz}), 104.3(\mathrm{~d}, J=22.5 \mathrm{~Hz})$, $104.2(\mathrm{~d}, J=22.0 \mathrm{~Hz}), 104.0(\mathrm{~d}, J=21.8 \mathrm{~Hz}), 69.0(\mathrm{~d}, J=7.1 \mathrm{~Hz}), 68.6(\mathrm{~d}, J=7.2 \mathrm{~Hz}), 56.8$ $(\mathrm{d}, J=155.0 \mathrm{~Hz}), 46.5(\mathrm{dd}, J=7.8,5.3 \mathrm{~Hz}), 23.9(\mathrm{~d}, J=5.6 \mathrm{~Hz}), 23.6(\mathrm{~d}, J=6.1 \mathrm{~Hz}), 9.9,9.8$; ${ }^{19} \mathrm{~F}$ NMR (565 MHz, $\left.\mathrm{CDCl}_{3}\right) \delta-108.62,-109.17,-109.35,-109.65 ;{ }^{31} \mathrm{P}$ NMR $(243 \mathrm{MHz}$, $\left.\mathrm{CDCl}_{3}\right) \delta$ 18.09; HRMS (ESI): $m / z$ calcd. for $\mathrm{C}_{22} \mathrm{H}_{23} \mathrm{~F}_{4} \mathrm{~N}_{2} \mathrm{NaO}_{4} \mathrm{P}[\mathrm{M}+\mathrm{Na}]^{+}:$509.1224, found: 509.1227.

Dipropyl ((3S, 4R)-4,6-bis(2-chloro-4-fluorophenyl)-5-oxo-2,3,4,5-tetrahydropyridazin3-yl)phosphonate (3qc)

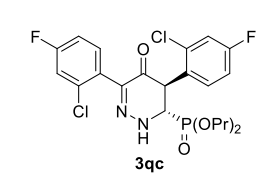

Isolated by silica gel column chromatography (petroleum ether : acetone $=$ $8: 1 \sim 1: 1, \mathrm{v} / \mathrm{v})$ to afford a yellow oil; (50.3 mg, 97\% yield, 91\% ee, >99:1 $d r)$, $[\alpha]_{\mathrm{D}}^{20}=-154.0^{\circ}(c=1.0, \mathrm{DCM}) ; \mathrm{HPLC}\left(\right.$ Chiralpak IA, $n$-hexane $/{ }^{i} \mathrm{PrOH}=$ $80 / 20$, flow rate $=0.5 \mathrm{~mL} / \mathrm{min}, \mathrm{I}=254 \mathrm{~nm}$ ): $t_{\mathrm{R}}=29.2 \mathrm{~min}$ (major), $35.7 \mathrm{~min}$ (minor); ${ }^{1} \mathrm{H}$ NMR (600 MHz, DMSO- $\left.d_{6}\right) \delta 10.08$ (brs, 1H), 7.51 (dd, $\left.J=8.8,2.8 \mathrm{~Hz}, 1 \mathrm{H}\right), 7.44-7.39$ (m, $2 \mathrm{H}), 7.33(\mathrm{dd}, J=8.6,6.3 \mathrm{~Hz}, 1 \mathrm{H}), 7.29(\mathrm{td}, J=8.5,2.8 \mathrm{~Hz}, 1 \mathrm{H}), 7.24(\mathrm{td}, J=8.5,2.7 \mathrm{~Hz}$, $1 \mathrm{H}), 4.70(\mathrm{dd}, J=14.5,6.7 \mathrm{~Hz}, 1 \mathrm{H}), 4.42(\mathrm{dd}, J=12.0,6.6 \mathrm{~Hz}, 1 \mathrm{H}), 3.94(\mathrm{dqt}, J=14.2,10.4$, 
$6.7 \mathrm{~Hz}, 4 \mathrm{H}), 1.62-1.51(\mathrm{~m}, 4 \mathrm{H}), 0.87-0.81(\mathrm{~m}, 6 \mathrm{H}) ;{ }^{13} \mathrm{C}$ NMR $\left(151 \mathrm{MHz}, \mathrm{DMSO}-d_{6}\right) \delta 180.6$ $(\mathrm{d}, J=4.0 \mathrm{~Hz}), 161.6(\mathrm{~d}, J=248.2 \mathrm{~Hz}), 161.2(\mathrm{~d}, J=248.1 \mathrm{~Hz}), 136.3,134.0(\mathrm{dd}, J=33.5$, $10.6 \mathrm{~Hz}), 133.4$ (d, $J=9.0 \mathrm{~Hz}), 131.1$ (d, $J=9.0 \mathrm{~Hz}), 130.8-129.2(\mathrm{~m}), 117.0$ (d, $J=25.2$ $\mathrm{Hz}), 116.5(\mathrm{~d}, J=25.0 \mathrm{~Hz}), 114.9(\mathrm{~d}, J=21.3 \mathrm{~Hz}), 114.1(\mathrm{~d}, J=21.0 \mathrm{~Hz}), 68.2(\mathrm{~d}, J=7.1$ Hz), $68.0(\mathrm{~d}, J=7.1 \mathrm{~Hz}), 55.7(\mathrm{~d}, J=148.5 \mathrm{~Hz}), 44.1,23.4(\mathrm{~d}, J=6.2 \mathrm{~Hz}), 23.3$ (d, $J=5.9$ $\mathrm{Hz}), 9.9,9.8 ;{ }^{19} \mathrm{~F}$ NMR (565 MHz, $\left.\mathrm{CDCl}_{3}\right) \delta-110.89,-111.44 ;{ }^{31} \mathrm{P}$ NMR $(243 \mathrm{MHz}$, DMSO- $\left.d_{6}\right) \delta$ 19.65; HRMS (ESI): $m / z$ calcd. for $\mathrm{C}_{22} \mathrm{H}_{23} \mathrm{Cl}_{2} \mathrm{~F}_{2} \mathrm{~N}_{2} \mathrm{O}_{4} \mathrm{P}[\mathrm{M}+\mathrm{Na}]^{+}:$518.0741, found: 518.0740 .

Dipropyl ((3S, 4R)-4,6-bis(2-bromo-4-fluorophenyl)-5-oxo-2,3,4,5-tetrahydropyridazin3-yl)phosphonate (3rc)

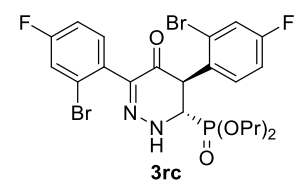

Isolated by silica gel column chromatography $($ petroleum ether $:$ acetone $=$ $8: 1 \sim 1: 1, \mathrm{v} / \mathrm{v})$ to afford a colorless oil $(54.7 \mathrm{mg}, 75 \%$ yield, $90 \%$ $e e,>99: 1 d r) ;[\alpha]_{\mathrm{D}}^{20}=-196.9^{\circ}(c=1.0, \mathrm{DCM})$; HPLC (Chiralpak IA, $n$-hexane $/{ }^{i} \mathrm{PrOH}=80 / 20$, flow rate $=0.5 \mathrm{~mL} / \mathrm{min}, \mathrm{I}=254 \mathrm{~nm}$ ): $t_{\mathrm{R}}=31.1 \mathrm{~min}$ (major), 33.5 $\min$ (minor); ${ }^{1} \mathrm{H}$ NMR $\left(600 \mathrm{MHz}, \mathrm{CDCl}_{3}\right) \delta 7.61(\mathrm{~s}, 1 \mathrm{H}), 7.36(\mathrm{dd}, J=8.1,2.7 \mathrm{~Hz}, 1 \mathrm{H}), 7.33$ $(\mathrm{dd}, J=8.3,2.6 \mathrm{~Hz}, 1 \mathrm{H}), 7.31-7.27(\mathrm{~m}, 2 \mathrm{H}), 7.07-7.01(\mathrm{~m}, 2 \mathrm{H}), 4.62(\mathrm{t}, J=11.7 \mathrm{~Hz}, 1 \mathrm{H})$, $4.55(\mathrm{t}, J=11.1 \mathrm{~Hz}, 1 \mathrm{H}), 4.10-4.01(\mathrm{~m}, 1 \mathrm{H}), 4.03-3.94(\mathrm{~m}, 1 \mathrm{H}), 3.90(\mathrm{q}, J=6.9 \mathrm{~Hz}, 2 \mathrm{H})$, $1.69(\mathrm{~h}, J=7.3 \mathrm{~Hz}, 2 \mathrm{H}), 1.53(\mathrm{~h}, J=7.2 \mathrm{~Hz}, 2 \mathrm{H}), 0.93(\mathrm{t}, J=7.4 \mathrm{~Hz}, 3 \mathrm{H}), 0.84$ (t, $J=7.4 \mathrm{~Hz}$, $3 \mathrm{H}) ;{ }^{13} \mathrm{C} \mathrm{NMR}\left(150 \mathrm{MHz}, \mathrm{CDCl}_{3}\right) \delta 182.0(\mathrm{~d}, J=9.7 \mathrm{~Hz}), 161.9(\mathrm{~d}, J=252.2 \mathrm{~Hz}), 162.3(\mathrm{~d}, J$ $=252.1 \mathrm{~Hz}), 142.0,132.7(\mathrm{~d}, J=8.7 \mathrm{~Hz}), 132.0,130.9(\mathrm{~d}, J=3.6 \mathrm{~Hz}), 130.6(\mathrm{dd}, J=6.7,3.6$ $\mathrm{Hz}), 124.9(\mathrm{~d}, J=9.2 \mathrm{~Hz}), 124.1(\mathrm{~d}, J=9.5 \mathrm{~Hz}), 120.7$ (d, $J=24.6 \mathrm{~Hz}), 120.1$ (d, $J=24.7$ Hz), 114.9 (d, $J=21.2 \mathrm{~Hz}), 114.4(\mathrm{~d}, J=21.1 \mathrm{~Hz}), 69.2$ (d, $J=7.1 \mathrm{~Hz}), 68.7(\mathrm{~d}, J=7.2 \mathrm{~Hz})$, $56.9(\mathrm{~d}, J=154.0 \mathrm{~Hz}), 50.1,24.0(\mathrm{~d}, J=5.5 \mathrm{~Hz}), 23.7(\mathrm{~d}, J=6.0 \mathrm{~Hz}), 10.0,9.9 ;{ }^{19} \mathrm{~F} \mathrm{NMR}$ $\left(565 \mathrm{MHz}, \mathrm{CDCl}_{3}\right) \delta-110.96,-111.53 ;{ }^{31} \mathrm{P} \mathrm{NMR}\left(243 \mathrm{MHz}, \mathrm{CDCl}_{3}\right) \delta 18.37 ; \mathrm{HRMS}$ (ESI): $m / z$ calcd. for $\mathrm{C}_{22} \mathrm{H}_{23} \mathrm{Br}_{2} \mathrm{~F}_{2} \mathrm{~N}_{2} \mathrm{NaO}_{4} \mathrm{P}[\mathrm{M}+\mathrm{Na}]^{+}$: 628.9622, found: 628.9623 .

Dipropyl ((3S, 4R)-4,6-bis(4-fluoro-2-methylphenyl)-5-oxo-2,3,4,5-tetrahydropyridazin3-yl)phosphonate (3sc)

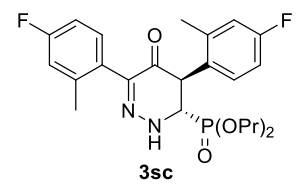

Isolated by silica gel column chromatography (petroleum ether : acetone $=$ $8: 1 \sim 1: 1, \mathrm{v} / \mathrm{v})$ to afford a yellow oil $(26.2 \mathrm{mg}, 55 \%$ yield, $94 \%$ ee, $>99: 1$ $d r) ;[\alpha]_{\mathrm{D}}^{20}=-220.9^{\circ}(c=1.0, \mathrm{DCM}) ;$ HPLC (Chiralpak IA, $n$-hexane $/{ }^{i} \mathrm{PrOH}=80 / 20$, flow rate $=0.5 \mathrm{~mL} / \mathrm{min}, \mathrm{I}=254 \mathrm{~nm}$ ): $t_{\mathrm{R}}=24.0 \mathrm{~min}$ (major), 18.6 min (minor); ${ }^{1} \mathrm{H}$ NMR (600 MHz, $\left.\mathrm{CDCl}_{3}\right) \delta 7.28(\mathrm{~s}, 1 \mathrm{H}), 7.27-7.22(\mathrm{~m}, 1 \mathrm{H}), 7.17$ (dd, $J=8.4$, $6.0 \mathrm{~Hz}, 1 \mathrm{H}), 6.93(\mathrm{dd}, J=9.6,2.7 \mathrm{~Hz}, 1 \mathrm{H}), 6.93-6.83(\mathrm{~m}, 3 \mathrm{H}), 4.41-4.31$ (m, 2H), 4.02-3.91 
(m, 3H), 3.85-3.77 (m, 1H), $2.42(\mathrm{~s}, 3 \mathrm{H}), 2.16(\mathrm{~s}, 3 \mathrm{H}), 1.68-1.60(\mathrm{~m}, 2 \mathrm{H}), 1.64-1.55(\mathrm{~m}, 2 \mathrm{H})$, 0.94-0.86 (m, 6H); ${ }^{13} \mathrm{C}$ NMR (151 MHz, $\left.\mathrm{CDCl}_{3}\right) \delta 183.8(\mathrm{~d}, J=7.7 \mathrm{~Hz}), 162.7$ (d, $J=246.9$ $\mathrm{Hz}), 162.1(\mathrm{~d}, J=247.0 \mathrm{~Hz}), 142.0,139.8$ (d, $J=8.0 \mathrm{~Hz}), 139.6(\mathrm{~d}, J=7.8 \mathrm{~Hz}), 131.6(\mathrm{~d}, J=$ $8.6 \mathrm{~Hz}), 130.5-130.2(\mathrm{~m}), 129.4(\mathrm{~d}, J=2.8 \mathrm{~Hz}), 129.3(\mathrm{~d}, J=8.4 \mathrm{~Hz}), 117.6(\mathrm{~d}, J=21.2 \mathrm{~Hz})$, $117.0(\mathrm{~d}, J=21.2 \mathrm{~Hz}), 113.4(\mathrm{~d}, J=21.2 \mathrm{~Hz}), 112.4(\mathrm{~d}, J=21.3 \mathrm{~Hz}), 68.9(\mathrm{~d}, J=7.2 \mathrm{~Hz})$, $68.6(\mathrm{~d}, J=7.3 \mathrm{~Hz}), 57.9$ (d, $J=152.9 \mathrm{~Hz}), 45.9,23.9(\mathrm{~d}, J=5.5 \mathrm{~Hz}), 23.8(\mathrm{~d}, J=5.9 \mathrm{~Hz})$, 20.2, 20.2, 9.9; ${ }^{19} \mathrm{~F}$ NMR (565 MHz, $\left.\mathrm{CDCl}_{3}\right) \delta-114.13,-114.49 ;{ }^{31} \mathrm{P}$ NMR (243 MHz, $\mathrm{CDCl}_{3}$ ) $\delta$ 19.47; HRMS (ESI): $m / z$ calcd. for $\mathrm{C}_{24} \mathrm{H}_{29} \mathrm{~F}_{2} \mathrm{~N}_{2} \mathrm{NaO}_{4} \mathrm{P}[\mathrm{M}+\mathrm{Na}]^{+}:$501.1725, found: 501.1729 .

Diisopropyl ((3S, 4R)-5-oxo-4,6-diphenyl-2,3,4,5-tetrahydropyridazin-3-yl)phosphonate (3ad)

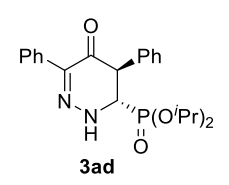

Isolated by silica gel column chromatography (petroleum ether : acetone $=8: 1$

$\sim 1: 1, \mathrm{v} / \mathrm{v})$ to afford a yellow solid $(24.8 \mathrm{mg}, 60 \%$ yield, 79\% ee, $>99: 1 \mathrm{dr}$ ), $\mathrm{mp} 145.1-146.8{ }^{\circ} \mathrm{C} ;[\alpha]_{\mathrm{D}}^{20}=-85.71^{\circ}(c=1.0, \mathrm{DCM})$; HPLC (Chiralpak IA, $n$-hexane $/{ }^{i} \mathrm{PrOH}=80 / 20$, flow rate $=0.5 \mathrm{~mL} / \mathrm{min}, \mathrm{I}=254 \mathrm{~nm}$ ): $t_{\mathrm{R}}=20.9 \mathrm{~min}$ (major), 16.5 min (minor); ${ }^{1} \mathrm{H}$ NMR (600 MHz, $\left.\mathrm{CDCl}_{3}\right) \delta 7.73-7.69(\mathrm{~m}, 3 \mathrm{H}), 7.36-7.26(\mathrm{~m}, 8 \mathrm{H}), 4.75(\mathrm{~h}, J$ $=6.3 \mathrm{~Hz}, 1 \mathrm{H}), 4.66(\mathrm{~h}, J=6.2 \mathrm{~Hz}, 1 \mathrm{H}), 4.26(\mathrm{dd}, J=12.5,6.5 \mathrm{~Hz}, 1 \mathrm{H}), 4.05(\mathrm{dd}, J=11.6,6.4$ $\mathrm{Hz}, 1 \mathrm{H}), 1.32(\mathrm{~d}, J=6.1 \mathrm{~Hz}, 3 \mathrm{H}), 1.27(\mathrm{~d}, J=6.2 \mathrm{~Hz}, 3 \mathrm{H}), 1.23(\mathrm{~d}, J=6.2 \mathrm{~Hz}, 2 \mathrm{H}), 1.20(\mathrm{~d}, J$ $=6.1 \mathrm{~Hz}, 3 \mathrm{H}) ;{ }^{13} \mathrm{C} \mathrm{NMR}\left(150 \mathrm{MHz}, \mathrm{CDCl}_{3}\right) \delta 184.2(\mathrm{~d}, J=4.4 \mathrm{~Hz}), 139.3(\mathrm{~d}, J=2.5 \mathrm{~Hz})$, $136.3(\mathrm{~d}, J=13.2 \mathrm{~Hz}), 134.3,129.1,128.4,128.2,128.1,128.0,127.8,72.7$ (d, $J=7.6 \mathrm{~Hz})$, $72.3(\mathrm{~d}, J=7.6 \mathrm{~Hz}), 58.9(\mathrm{~d}, J=151.7 \mathrm{~Hz}), 49.9(\mathrm{~d}, J=2.4 \mathrm{~Hz}), 24.2,24.1(\mathrm{~d}, J=4.6 \mathrm{~Hz})$, $24.0(\mathrm{~d}, J=4.5 \mathrm{~Hz}), 24.0(\mathrm{~d}, J=4.0 \mathrm{~Hz}) ;{ }^{31} \mathrm{P} \mathrm{NMR}\left(243 \mathrm{MHz}, \mathrm{CDCl}_{3}\right) \delta 17.91$; HRMS (ESI): $m / z$ calcd. for $\mathrm{C}_{22} \mathrm{H}_{27} \mathrm{~N}_{2} \mathrm{NaO}_{4} \mathrm{P}[\mathrm{M}+\mathrm{Na}]^{+}:$437.1601, found: 437.1600 .

Di-tert-butyl $((3 S, 4 R)-5$-oxo-4,6-diphenyl-2,3,4,5-tetrahydropyridazin-3-yl)phosphonat e (3ae)

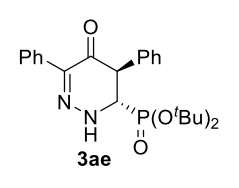

Isolated by silica gel column chromatography (petroleum ether : acetone $=8: 1$

$\sim 1: 1, \mathrm{v} / \mathrm{v})$ to afford a yellow solid $(9.3 \mathrm{mg}, 21 \%$ yield, 30\% ee, $>99: 1 \mathrm{dr}), \mathrm{mp}$ 109.1-110.6 ${ }^{\circ} \mathrm{C} ;[\alpha]_{\mathrm{D}}^{20}=-36.0^{\circ}(c=1.0$, DCM); HPLC (Chiralpak IC, $n$-hexane ${ }^{i} \mathrm{PrOH}=70 / 30$, flow rate $=0.5 \mathrm{~mL} / \mathrm{min}, \mathrm{I}=254 \mathrm{~nm}$ ): $t_{\mathrm{R}}=10.9 \mathrm{~min}$ (major), 28.0 min (minor); ${ }^{1} \mathrm{H}$ NMR (600 MHz, $\left.\mathrm{CDCl}_{3}\right) \delta 7.98(\mathrm{~s}, 1 \mathrm{H}), 7.69(\mathrm{~d}, J=7.9 \mathrm{~Hz}, 2 \mathrm{H}), 7.32-7.19$ (m, 8H), 4.07 (dd, $J=10.4,4.2 \mathrm{~Hz}, 2 \mathrm{H}), 3.98$ (dd, $J=11.9,4.8 \mathrm{~Hz}, 1 \mathrm{H}), 1.44$ (s, 9H), 1.38 (s, $9 \mathrm{H}) ;{ }^{13} \mathrm{C} \mathrm{NMR}\left(150 \mathrm{MHz}, \mathrm{CDCl}_{3}\right) \delta 184.1(\mathrm{~d}, J=2.4 \mathrm{~Hz}), 137.9(\mathrm{t}, J=3.3 \mathrm{~Hz}), 137.1(\mathrm{~d}, J=$ $14.8 \mathrm{~Hz}), 134.6,129.2,128.1,128.0,127.7,127.6,85.0$ (d, $J=10.1 \mathrm{~Hz}), 84.3$ (d, $J=9.3 \mathrm{~Hz})$, 
$60.7(\mathrm{~d}, J=154.1 \mathrm{~Hz}), 49.7(\mathrm{~d}, J=2.8 \mathrm{~Hz}), 30.5(\mathrm{~d}, J=4.1 \mathrm{~Hz}), 30.4(\mathrm{~d}, J=3.7 \mathrm{~Hz}) ;{ }^{31} \mathrm{P}$ NMR $\left(243 \mathrm{MHz}, \mathrm{CDCl}_{3}\right) \delta$ 12.22; HRMS (ESI): $\mathrm{m} / z$ calcd. for $\mathrm{C}_{24} \mathrm{H}_{31} \mathrm{~N}_{2} \mathrm{NaO}_{4} \mathrm{P}[\mathrm{M}+\mathrm{Na}]^{+}$: 465.1914, found: 465.1915 .

\section{Dimethyl ((3S, 4R)-4,6-bis(4-fluorophenyl)-5-oxo-2,3,4,5-tetrahydropyridazin-3-yl)phos -phonate (3ba)}

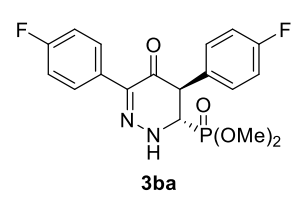

Isolated by silica gel column chromatography (petroleum ether $:$ acetone $=$ $8: 1 \sim 1: 1, \mathrm{v} / \mathrm{v})$ to afford a yellow solid $(37.8 \mathrm{mg}, 96 \%$ yield, $88 \%$ $e e,>99: 1 d r), \operatorname{mp~} 108.3-110.0^{\circ} \mathrm{C} ;[\alpha]_{\mathrm{D}}^{20}=-229.9^{\circ}(c=1.0, \mathrm{DCM}) ; \mathrm{HPLC}$ (Chiralpak ASH, $n$-hexane $/{ }^{i} \operatorname{PrOH}=70 / 30$, flow rate $=0.5 \mathrm{~mL} / \mathrm{min}, \mathrm{I}=254 \mathrm{~nm}$ ): $t_{\mathrm{R}}=20.0 \mathrm{~min}$ (major), $25.9 \mathrm{~min}$ (minor); ${ }^{1} \mathrm{H}$ NMR (600 MHz, $\left.\mathrm{CDCl}_{3}\right) \delta 7.71-7.68(\mathrm{~m}, 2 \mathrm{H}), 7.54(\mathrm{~s}, 1 \mathrm{H})$, 7.29-7.26 (m, 2H), $7.06(\mathrm{t}, J=8.6 \mathrm{~Hz}, 2 \mathrm{H}), 7.02(\mathrm{t}, J=8.8 \mathrm{~Hz}, 2 \mathrm{H}), 4.36(\mathrm{dd}, J=12.4,8.4$ $\mathrm{Hz}, 1 \mathrm{H}), 4.05(\mathrm{dd}, J=11.0,8.2 \mathrm{~Hz}, 1 \mathrm{H}), 3.69$ (d, $J=10.5 \mathrm{~Hz}, 3 \mathrm{H}), 3.67$ (d, $J=10.4 \mathrm{~Hz}, 3 \mathrm{H})$; ${ }^{13} \mathrm{C} \mathrm{NMR}\left(150 \mathrm{MHz}, \mathrm{CDCl}_{3}\right) \delta 184.1(\mathrm{~d}, J=6.3 \mathrm{~Hz}), 162.9(\mathrm{~d}, J=248.0 \mathrm{~Hz}), 162.7(\mathrm{~d}, J=$ $247.6 \mathrm{~Hz}), 139.1,131.1(\mathrm{dd}, J=11.9,3.4 \mathrm{~Hz}), 130.3(\mathrm{~d}, J=8.1 \mathrm{~Hz}), 130.0(\mathrm{~d}, J=3.3 \mathrm{~Hz})$, $129.6(\mathrm{~d}, J=8.1 \mathrm{~Hz}), 116.1(\mathrm{~d}, J=21.8 \mathrm{~Hz}), 115.1(\mathrm{~d}, J=21.5 \mathrm{~Hz}), 58.0(\mathrm{~d}, J=151.8 \mathrm{~Hz})$, $54.0(\mathrm{~d}, J=7.0 \mathrm{~Hz}), 53.5(\mathrm{~d}, J=7.2 \mathrm{~Hz}), 49.3(\mathrm{~d}, J=2.4 \mathrm{~Hz}) ;{ }^{19} \mathrm{~F}$ NMR $\left(565 \mathrm{MHz}, \mathrm{CDCl}_{3}\right) \delta$ -113.36, -113.41; ${ }^{31} \mathrm{P}$ NMR (243 MHz, $\left.\mathrm{CDCl}_{3}\right) \delta$ 21.55; HRMS (ESI): $\mathrm{m} / z$ calcd. for $\mathrm{C}_{18} \mathrm{H}_{17} \mathrm{~F}_{2} \mathrm{~N}_{2} \mathrm{NaO}_{4} \mathrm{P}[\mathrm{M}+\mathrm{Na}]^{+}:$417.0786, found: 417.0781 .

Diethyl ((3S, 4R)-4,6-bis(4-fluorophenyl)-5-oxo-2,3,4,5-tetrahydropyridazin-3-yl)phosp h-onate (3bb)

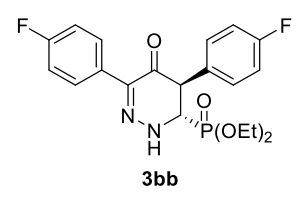

Isolated by silica gel column chromatography (petroleum ether $:$ acetone $=$ $8: 1 \sim 1: 1, \mathrm{v} / \mathrm{v})$ to afford a yellow solid $(38.8 \mathrm{mg}, 92 \%$ yield, $93 \%$ $e e,>99: 1 d r), \operatorname{mp} 110.8-112.6^{\circ} \mathrm{C} ;[\alpha]_{\mathrm{D}}^{20}=-233.3^{\circ}(c=1.0, \mathrm{DCM}) ; \mathrm{HPLC}$ (Chiralpak IA, $n$-hexane $/{ }^{i} \mathrm{PrOH}=80 / 20$, flow rate $=0.5 \mathrm{~mL} / \mathrm{min}, \mathrm{I}=254 \mathrm{~nm}$ ): $t_{\mathrm{R}}=24.7 \mathrm{~min}$ (major), $20.3 \mathrm{~min}$ (minor); ${ }^{1} \mathrm{H}$ NMR $\left(600 \mathrm{MHz}, \mathrm{CDCl}_{3}\right) \delta 7.84$ (s, 1H), 7.69 (dd, $J=8.5,5.5$ $\mathrm{Hz}, 2 \mathrm{H}), 7.27(\mathrm{dd}, J=7.3,4.1 \mathrm{~Hz}, 2 \mathrm{H}), 7.04(\mathrm{t}, J=8.5 \mathrm{~Hz}, 2 \mathrm{H}), 7.00(\mathrm{t}, J=8.6 \mathrm{~Hz}, 2 \mathrm{H}), 4.32$ $(\mathrm{dd}, J=12.8,7.6 \mathrm{~Hz}, 1 \mathrm{H}), 4.17-4.07(\mathrm{~m}, 1 \mathrm{H}), 4.09-3.98(\mathrm{~m}, 4 \mathrm{H}), 1.26(\mathrm{t}, J=7.1 \mathrm{~Hz}, 3 \mathrm{H})$, $1.22(\mathrm{t}, J=7.1 \mathrm{~Hz}, 3 \mathrm{H}) ;{ }^{13} \mathrm{C} \mathrm{NMR}\left(150 \mathrm{MHz}, \mathrm{CDCl}_{3}\right) \delta 184.1(\mathrm{~d}, J=5.6 \mathrm{~Hz}), 162.8(\mathrm{~d}, J=$ $247.7 \mathrm{~Hz}), 162.7(\mathrm{~d}, J=247.4 \mathrm{~Hz}), 138.6,131.4(\mathrm{dd}, J=12.3,3.3 \mathrm{~Hz}), 130.2(\mathrm{~d}, J=8.2 \mathrm{~Hz})$, $130.2(\mathrm{~d}, J=3.3 \mathrm{~Hz}), 129.5(\mathrm{~d}, J=8.1 \mathrm{~Hz}), 116.0(\mathrm{~d}, J=21.6 \mathrm{~Hz}), 115.0(\mathrm{~d}, J=21.4 \mathrm{~Hz})$, $63.7(\mathrm{~d}, J=7.0 \mathrm{~Hz}), 63.3(\mathrm{~d}, J=7.4 \mathrm{~Hz}), 58.2(\mathrm{~d}, J=151.4 \mathrm{~Hz}), 49.2(\mathrm{~d}, J=2.4 \mathrm{~Hz}), 16.5(\mathrm{~d}$, $J=5.6 \mathrm{~Hz}), 16.3(\mathrm{~d}, J=5.7 \mathrm{~Hz}) ;{ }^{19} \mathrm{~F}$ NMR $\left(565 \mathrm{MHz}, \mathrm{CDCl}_{3}\right) \delta-113.65,-113.67 ;{ }^{31} \mathrm{P} \mathrm{NMR}$ $\left(243 \mathrm{MHz}, \mathrm{CDCl}_{3}\right) \delta$ 19.31; HRMS (ESI): $m / z$ calcd. for $\mathrm{C}_{20} \mathrm{H}_{21} \mathrm{~F}_{2} \mathrm{~N}_{2} \mathrm{NaO}_{4} \mathrm{P}[\mathrm{M}+\mathrm{Na}]^{+}$: 
445.1099, found: 445.1098 .

Dipropyl ((3S, 4R)-6-(4-fluorophenyl)-5-oxo-4-(o-tolyl)-2,3,4,5-tetrahydropyridazin-3-yl) phosphonate (3tc)

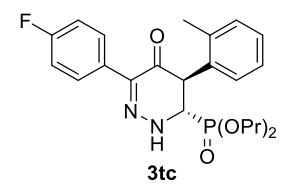

Isolated by silica gel column chromatography $(\mathrm{DCM} / \mathrm{THF}=30: 1, \mathrm{v} / \mathrm{v})$ to afford a yellow oil $(9.8 \mathrm{mg}, 22 \%$ yield, $95 \% e e,>99: 1 \mathrm{dr}) ; \mathrm{R}_{\mathrm{f}}=0.2$ $(\mathrm{DCM} / \mathrm{THF}=30: 1) ;[\alpha]_{\mathrm{D}}^{20}=-276.7^{\circ}(c=1.0, \mathrm{DCM}) ;$ HPLC $($ Chiralpak IA, $n$-hexane $/{ }^{i} \mathrm{PrOH}=80 / 20$, flow rate $=0.5 \mathrm{~mL} / \mathrm{min}, \mathrm{I}=254 \mathrm{~nm}$ ): $t_{\mathrm{R}}=19.4 \mathrm{~min}$ (major), 16.3 min (minor); ${ }^{1} \mathrm{H}$ NMR (600 MHz, $\left.\mathrm{CDCl}_{3}\right) \delta 7.76-7.73(\mathrm{~m}, 2 \mathrm{H}), 7.39(\mathrm{~s}, 1 \mathrm{H}), 7.24-7.18(\mathrm{~m}$, 2H), 7.17 (d, $J=4.1 \mathrm{~Hz}, 2 \mathrm{H}), 7.05-7.00(\mathrm{~m}, 2 \mathrm{H}), 4.39-4.29(\mathrm{~m}, 2 \mathrm{H}), 4.00-3.89(\mathrm{~m}, 3 \mathrm{H})$, 3.79-3.72 (m, 1H), $2.43(\mathrm{~s}, 3 \mathrm{H}), 1.58(\mathrm{~h}, J=7.1 \mathrm{~Hz}, 4 \mathrm{H}), 0.89-0.84(\mathrm{~m}, 6 \mathrm{H}) ;{ }^{13} \mathrm{C}$ NMR $(151$ $\left.\mathrm{MHz}, \mathrm{CDCl}_{3}\right) \delta 184.9(\mathrm{~d}, J=6.2 \mathrm{~Hz}), 162.9(\mathrm{~d}, J=247.5 \mathrm{~Hz}), 139.4,136.8,134.8(\mathrm{~d}, J=10.3$ $\mathrm{Hz}), 131.2,130.2$ (d, $J=3.2 \mathrm{~Hz}), 129.7$ (d, $J=8.0 \mathrm{~Hz}), 128.1,127.7,126.8,115.0$ (d, $J=21.5$ Hz), 69.1 (d, $J=7.2 \mathrm{~Hz}), 68.6(\mathrm{~d}, J=7.7 \mathrm{~Hz}), 58.1$ (d, $J=151.6 \mathrm{~Hz}), 46.4$ (d, $J=2.6 \mathrm{~Hz})$, $24.0(\mathrm{~d}, J=5.7 \mathrm{~Hz}), 23.9(\mathrm{~d}, J=6.2 \mathrm{~Hz}), 20.1,10.1,10.0 ;{ }^{19} \mathrm{~F}$ NMR $\left(565 \mathrm{MHz}, \mathrm{CDCl}_{3}\right) \delta$ -113.74; ${ }^{31} \mathrm{P}$ NMR (243 MHz, $\left.\mathrm{CDCl}_{3}\right) \delta$ 19.70; HRMS (ESI): $m / z$ calcd. for $\mathrm{C}_{23} \mathrm{H}_{28} \mathrm{FN}_{2} \mathrm{NaO}_{4} \mathrm{P}$ $[\mathrm{M}+\mathrm{Na}]^{+}:$469.1663, found: 469.1664 .

Dipropyl ((3S, 4R)-4-(4-fluorophenyl)-5-oxo-6-(o-tolyl)-2,3,4,5-tetrahydropyridazin-3-yl) phosphonate $\left(3^{\prime}\right.$ tc $)$

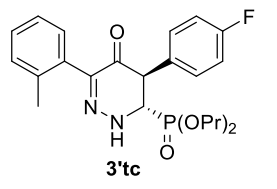

Isolated by silica gel column chromatography $(\mathrm{DCM} / \mathrm{THF}=60: 1, \mathrm{v} / \mathrm{v})$ to afford a yellow oil $(9.8 \mathrm{mg}, 22 \%$ yield, $95 \%$ ee, >99:1 $d r) ; \mathrm{R}_{\mathrm{f}}=0.6$ $(\mathrm{DCM} / \mathrm{THF}=30: 1) ;[\alpha]_{\mathrm{D}}^{20}=-146.7^{\circ}(c=1.0, \mathrm{DCM}) ;$ HPLC $($ Chiralpak IC, $n$-hexane $/ \mathrm{PrOH}=80 / 20$, flow rate $=0.5 \mathrm{~mL} / \mathrm{min}, \mathrm{I}=254 \mathrm{~nm}$ ): $t_{\mathrm{R}}=26.2 \mathrm{~min}$ (major), 39.4 min (minor); ${ }^{1} \mathrm{H}$ NMR (600 MHz, $\left.\mathrm{CDCl}_{3}\right) \delta 7.45$ (s, 1H), 7.43-7.39 (m, 2H), 7.34-7.29 (m, 1H), 7.27-7.22 (m, 3H), 7.17-7.12 (m, 2H), 4.43 (dd, $J=12.7,8.3 \mathrm{~Hz}, 1 \mathrm{H}), 4.19-4.09$ (m, 2H), 4.08-3.98 (m, 3H), 2.20 (s, 3H), $1.76(\mathrm{~h}, J=7.2 \mathrm{~Hz}, 2 \mathrm{H}), 1.67$ (h, $J=7.1 \mathrm{~Hz}, 2 \mathrm{H}), 1.02$ $(\mathrm{t}, J=7.4 \mathrm{~Hz}, 3 \mathrm{H}), 0.96(\mathrm{t}, J=7.4 \mathrm{~Hz}, 3 \mathrm{H}) ;{ }^{13} \mathrm{C} \mathrm{NMR}\left(151 \mathrm{MHz}, \mathrm{CDCl}_{3}\right) \delta 183.5(\mathrm{~d}, J=6.4$ Hz), $162.8(\mathrm{~d}, J=247.6 \mathrm{~Hz}), 142.7,137.2,133.5,131.7-131.3(\mathrm{~m}), 130.5,130.4$ (d, $J=8.1$ Hz), 130.0, 128.7, 125.6, $116.0(\mathrm{~d}, J=21.6 \mathrm{~Hz}), 69.0(\mathrm{~d}, J=7.2 \mathrm{~Hz}), 68.8(\mathrm{~d}, J=7.3 \mathrm{~Hz})$, $58.3(\mathrm{~d}, J=152.1 \mathrm{~Hz}), 49.5(\mathrm{~d}, J=2.5 \mathrm{~Hz}), 24.1(\mathrm{~d}, J=5.4 \mathrm{~Hz}), 23.9(\mathrm{~d}, J=5.8 \mathrm{~Hz}), 20.1$, 10.1, 10.0; ${ }^{19} \mathrm{~F}$ NMR (565 MHz, $\left.\mathrm{CDCl}_{3}\right) \delta-113.72 ;{ }^{31} \mathrm{P}$ NMR $\left(243 \mathrm{MHz}, \mathrm{CDCl}_{3}\right) \delta$ 19.48; HRMS (ESI): $m / z$ calcd. for $\mathrm{C}_{23} \mathrm{H}_{28} \mathrm{FN}_{2} \mathrm{NaO}_{4} \mathrm{P}$ [M+Na] $]^{+}$: 469.1663, found: 469.1666 . 


\section{Dipropyl ((3S, 4R)-4-(4-fluorophenyl)-6-(4-methoxyphenyl)-5-oxo-2,3,4,5-tetrahydropy}

r-idazin-3-yl)phosphonate (3uc)

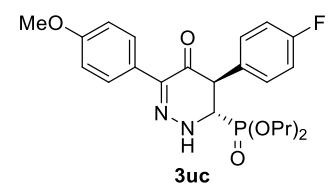

Isolated by silica gel column chromatography $(\mathrm{PE} / \mathrm{EA}=1: 1, \mathrm{v} / \mathrm{v})$ to afford a yellow oil (14.8 mg, 32\% yield, 92\% ee, >99:1 dr); $\mathrm{R}_{\mathrm{f}}=0.4$ $(\mathrm{PE} / \mathrm{EA}=1: 1) ;[\alpha]_{\mathrm{D}}^{20}=-188.9^{\circ}(c=1.0, \mathrm{DCM}) ; \mathrm{HPLC}($ Chiralpak IC, $n$-hexane $/{ }^{i} \mathrm{PrOH}=80 / 20$, flow rate $\left.=0.5 \mathrm{~mL} / \mathrm{min}, \mathrm{I}=254 \mathrm{~nm}\right): t_{\mathrm{R}}=$ $38.2 \mathrm{~min}$ (major), $41.5 \mathrm{~min}$ (minor); ${ }^{1} \mathrm{H}$ NMR (600 MHz, Acetone- $\left.d_{6}\right) \delta 8.78(\mathrm{~s}, 1 \mathrm{H}), 7.67$ (d, $J=8.9 \mathrm{~Hz}, 2 \mathrm{H}), 7.45-7.40(\mathrm{~m}, 2 \mathrm{H}), 7.17-7.10(\mathrm{~m}, 2 \mathrm{H}), 6.86(\mathrm{~d}, J=8.9 \mathrm{~Hz}, 2 \mathrm{H}), 4.65-4.56$ $(\mathrm{m}, 1 \mathrm{H}), 4.07-3.91(\mathrm{~m}, 5 \mathrm{H}), 3.79(\mathrm{~s}, 3 \mathrm{H}), 1.69-1.48(\mathrm{~m}, 4 \mathrm{H}), 0.93-0.82(\mathrm{~m}, 6 \mathrm{H}) ;{ }^{13} \mathrm{C} \mathrm{NMR}$ $\left(151 \mathrm{MHz}\right.$, Acetone- $\left.d_{6}\right) \delta 184.4,163.2(\mathrm{~d}, J=244.6 \mathrm{~Hz}), 160.2,137.9(\mathrm{~d}, J=2.6 \mathrm{~Hz}), 134.0$ $(\mathrm{dd}, J=16.1,3.2 \mathrm{~Hz}), 130.9(\mathrm{~d}, J=8.1 \mathrm{~Hz}), 129.5,128.6,116.3(\mathrm{~d}, J=21.6 \mathrm{~Hz}), 113.9,69.0$ $(\mathrm{d}, J=4.4 \mathrm{~Hz}), 68.9$ (d, $J=4.7 \mathrm{~Hz}), 58.2(\mathrm{~d}, J=148.7 \mathrm{~Hz}), 55.5,49.1,24.6(\mathrm{~d}, J=6.1 \mathrm{~Hz})$, $24.5(\mathrm{~d}, J=6.2 \mathrm{~Hz}), 10.3,10.3 ;{ }^{19} \mathrm{~F}$ NMR $\left(565 \mathrm{MHz}, \mathrm{CDCl}_{3}\right) \delta-113.91 ;{ }^{31} \mathrm{P}$ NMR $(243 \mathrm{MHz}$, Acetone- $\left.d_{6}\right) \delta$ 20.18; HRMS (ESI): $m / z$ calcd. for $\mathrm{C}_{23} \mathrm{H}_{28} \mathrm{FN}_{2} \mathrm{NaO}_{5} \mathrm{P}[\mathrm{M}+\mathrm{Na}]^{+}: 485.1612$, found: 485.1610 .

Dipropyl ((3S, 4R)-6-(4-fluorophenyl)-4-(4-methoxyphenyl)-5-oxo-2,3,4,5-tetrahydropy r-idazin-3-yl)phosphonate (3'uc)

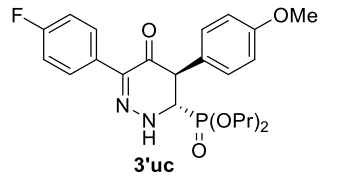

Isolated by silica gel column chromatography $(\mathrm{PE} / \mathrm{EA}=1: 1, \mathrm{v} / \mathrm{v})$ to afford a yellow oil (10.2 mg, 22\% yield, $80 \% e e,>99: 1 d r) ; \mathrm{R}_{\mathrm{f}}=0.35$ $(\mathrm{PE} / \mathrm{EA}=1: 1) ;[\alpha]_{\mathrm{D}}^{20}=-147.13^{\circ}(c=1.0, \mathrm{DCM}) ; \mathrm{HPLC}$ (Chiralpak IC, $n$-hexane $/{ }^{i} \mathrm{PrOH}=80 / 20$, flow rate $=0.5 \mathrm{~mL} / \mathrm{min}, \mathrm{I}=254 \mathrm{~nm}$ ): $t_{\mathrm{R}}=25.5 \mathrm{~min}$ (major), 29.8 $\min$ (minor); ${ }^{1} \mathrm{H}$ NMR (600 MHz, Acetone- $\left.d_{6}\right) \delta 9.05$ (s, $\left.1 \mathrm{H}\right), 7.77$ (td, $J=6.8,5.7,2.3 \mathrm{~Hz}$, 2H), 7.34-7.28 (m, 2H), $7.06(\mathrm{td}, J=9.0,2.3 \mathrm{~Hz}, 2 \mathrm{H}), 6.99-6.87(\mathrm{~m}, 2 \mathrm{H}), 4.61(\mathrm{~d}, J=14.4$ $\mathrm{Hz}, 1 \mathrm{H}), 4.08-3.94(\mathrm{~m}, 5 \mathrm{H}), 3.77(\mathrm{~s}, 1 \mathrm{H}), 1.63(\mathrm{p}, J=7.2 \mathrm{~Hz}, 4 \mathrm{H}), 0.89$ (q, $J=5.6 \mathrm{~Hz}, 6 \mathrm{H})$; ${ }^{13} \mathrm{C}$ NMR $\left(151 \mathrm{MHz}\right.$, Acetone- $\left.d_{6}\right) \delta 184.5,163.1(\mathrm{~d}, J=244.6 \mathrm{~Hz}), 160.6,136.5(\mathrm{~d}, J=3.9$ Hz), 132.7 (d, $J=1.5 \mathrm{~Hz}), 130.1(\mathrm{~d}, J=7.9 \mathrm{~Hz}), 129.8,129.8,115.3,115.3$ (d, $J=21.4 \mathrm{~Hz})$, $69.1(\mathrm{~d}, J=4.7 \mathrm{~Hz}), 58.4(\mathrm{~d}, J=147.8 \mathrm{~Hz}), 55.7,48.5,24.7(\mathrm{~d}, J=3.6 \mathrm{~Hz}), 24.7$ (d, $J=3.3$ $\mathrm{Hz}), 10.5 ;{ }^{19} \mathrm{~F}$ NMR $\left(565 \mathrm{MHz}, \mathrm{CDCl}_{3}\right) \delta-113.92 ;{ }^{31} \mathrm{P}$ NMR (243 MHz, Acetone- $\left.d_{6}\right) \delta 20.48$; HRMS (ESI): $m / z$ calcd. for $\mathrm{C}_{23} \mathrm{H}_{28} \mathrm{FN}_{2} \mathrm{NaO}_{5} \mathrm{P}[\mathrm{M}+\mathrm{Na}]^{+}: 485.1612$, found 485.1610 . 


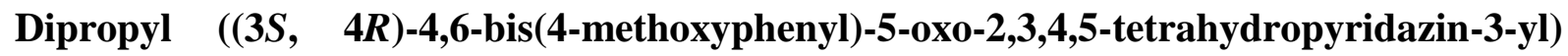
phosphonate (3vc)

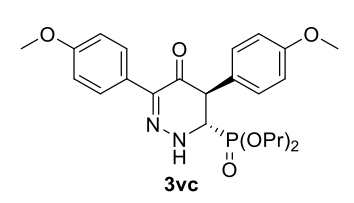

Isolated by silica gel column chromatography (DCM/acetone $=30: 1$, $\mathrm{v} / \mathrm{v})$ to afford a yellow oil $(6.6 \mathrm{mg}, 14 \%$ yield, $50 \% e e,>99: 1 d r) ; \mathrm{R}_{\mathrm{f}}=$ $0.3(\mathrm{DCM} /$ acetone $=30: 1) ;[\alpha]_{\mathrm{D}}^{20}=-56.7^{\circ}(c=1.0, \mathrm{DCM}) ; \mathrm{HPLC}$

(Chiralpak IA, $n$-hexane ${ }^{i} \mathrm{PrOH}=80 / 20$, flow rate $=0.5 \mathrm{~mL} / \mathrm{min}, \mathrm{I}=$ $254 \mathrm{~nm}$ ): $t_{\mathrm{R}}=52.7 \mathrm{~min}$ (major), $43.3 \mathrm{~min}$ (minor); ${ }^{1} \mathrm{H} \mathrm{NMR}\left(600 \mathrm{MHz}, \mathrm{CDCl}_{3}\right) \delta 7.66$ (d, $J=$ $8.9 \mathrm{~Hz}, 2 \mathrm{H}), 7.25-7.20(\mathrm{~m}, 3 \mathrm{H}), 6.90-6.85(\mathrm{~m}, 4 \mathrm{H}), 4.31(\mathrm{dd}, J=12.2,7.6 \mathrm{~Hz}, 1 \mathrm{H}), 4.05-$ $3.96(\mathrm{~m}, 2 \mathrm{H}), 3.96-3.89(\mathrm{~m}, 2 \mathrm{H}), 3.91-3.85(\mathrm{~m}, 1 \mathrm{H}), 3.81$ (s, 3H), $3.79(\mathrm{~s}, 3 \mathrm{H}), 1.66-1.53(\mathrm{~m}$, 4H), 0.92-0.84 (m, 6H); $\left.{ }^{13} \mathrm{C} \mathrm{NMR} \mathrm{(151} \mathrm{MHz,} \mathrm{CDCl}_{3}\right) \delta 159.5(\mathrm{~d}, J=21.1 \mathrm{~Hz}), 139.7(\mathrm{~d}, J=$ $2.1 \mathrm{~Hz}), 129.5,129.0,127.7(\mathrm{~d}, J=11.8 \mathrm{~Hz}), 126.7,114.4,113.4,68.8$ (d, $J=7.1 \mathrm{~Hz}), 68.4$ (d, $J=7.5 \mathrm{~Hz}), 58.4(\mathrm{~d}, J=150.3 \mathrm{~Hz}), 55.3,55.3,49.4,23.9(\mathrm{~d}, J=5.9 \mathrm{~Hz}), 23.8(\mathrm{~d}, J=5.8 \mathrm{~Hz})$, 10.0, 9.9; ${ }^{31} \mathrm{P}$ NMR (243 MHz, $\mathrm{CDCl}_{3}$ ) $\delta$ 19.82; HRMS (ESI): $m / z$ calcd. for $\mathrm{C}_{24} \mathrm{H}_{31} \mathrm{~N}_{2} \mathrm{NaO}_{6} \mathrm{P}$ $[\mathrm{M}+\mathrm{Na}]^{+}:$497.1812, found: 497.1814 .

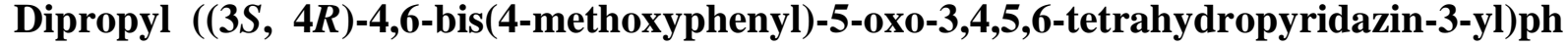 -osphonate $(9 \mathrm{vc})$}

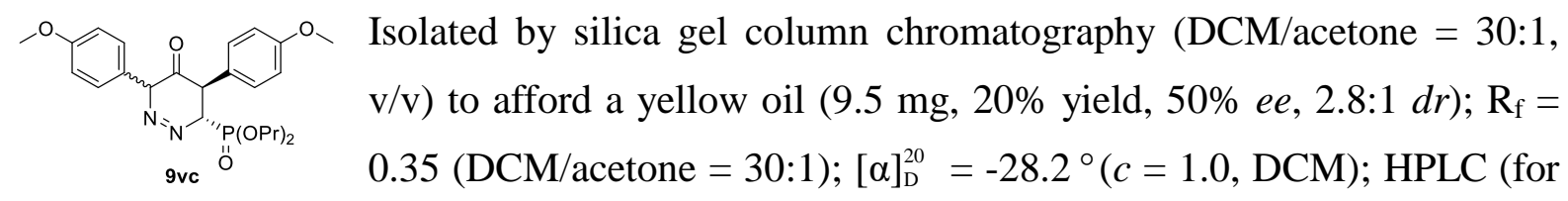
major diastereoisomer, Chiralpak IC, $n$-hexane $/{ }^{i} \mathrm{PrOH}=80 / 20$, flow rate $=0.5 \mathrm{~mL} / \mathrm{min}, \mathrm{I}=$ $254 \mathrm{~nm}$ ): $t_{\mathrm{R}}=59.2 \mathrm{~min}$ (major), $83.3 \mathrm{~min}$ (minor); ${ }^{1} \mathrm{H}$ NMR (for major diastereoisomers, 600 $\left.\mathrm{MHz}, \mathrm{CDCl}_{3}\right) \delta 7.89$ (s, 1H), 7.66-7.54 (m, 1H), 7.46-7.29 (m, 1H), 7.19-7.10 (m, 4H), 6.83$6.74(\mathrm{~m}, 3 \mathrm{H}), 4.27-4.17(\mathrm{~m}, 1 \mathrm{H}), 3.97-3.86(\mathrm{~m}, 2 \mathrm{H}), 3.84(\mathrm{t}, J=6.6 \mathrm{~Hz}, 2 \mathrm{H}), 3.83-3.78(\mathrm{~m}$, $3 \mathrm{H}), 3.76-3.68(\mathrm{~m}, 3 \mathrm{H}), 1.58-1.43(\mathrm{~m}, 4 \mathrm{H}), 0.85-0.74(\mathrm{~m}, 6 \mathrm{H}) ;{ }^{13} \mathrm{C} \mathrm{NMR}$ (for major diastereoisomers, $\left.150 \mathrm{MHz}, \mathrm{CDCl}_{3}\right) \delta 184.5$ (d, $\left.J=5.4 \mathrm{~Hz}\right), 159.5,155.7,139.9,137.8,129.4$, 129.0, 127.9, 114.5, 113.5, 111.2, 77.2, 68.9 (d, $J=7.2 \mathrm{~Hz}), 68.5(\mathrm{~d}, J=7.6 \mathrm{~Hz}), 58.3$ (d, $J=$ 150.6 Hz), 56.3, 55.3, 49.2, $23.9(\mathrm{~d}, J=6.0 \mathrm{~Hz}), 23.8(\mathrm{~d}, J=6.1 \mathrm{~Hz}), 10.0,9.9 ;{ }^{31} \mathrm{P}$ NMR $(243$ $\mathrm{MHz}, \mathrm{CDCl}_{3}$ ) $\delta 19.63$ (major); 19.30 (minor); HRMS (ESI): $\mathrm{m} / z$ calcd. for $\mathrm{C}_{24} \mathrm{H}_{31} \mathrm{~N}_{2} \mathrm{NaO}_{6} \mathrm{P}$ $[\mathrm{M}+\mathrm{Na}]^{+}:$497.1812, found: 497.1812.

The $d r$ of 9vc was determined by ${ }^{31} \mathrm{P}$ NMR $\left(243 \mathrm{MHz}, \mathrm{CDCl}_{3}\right)$. 


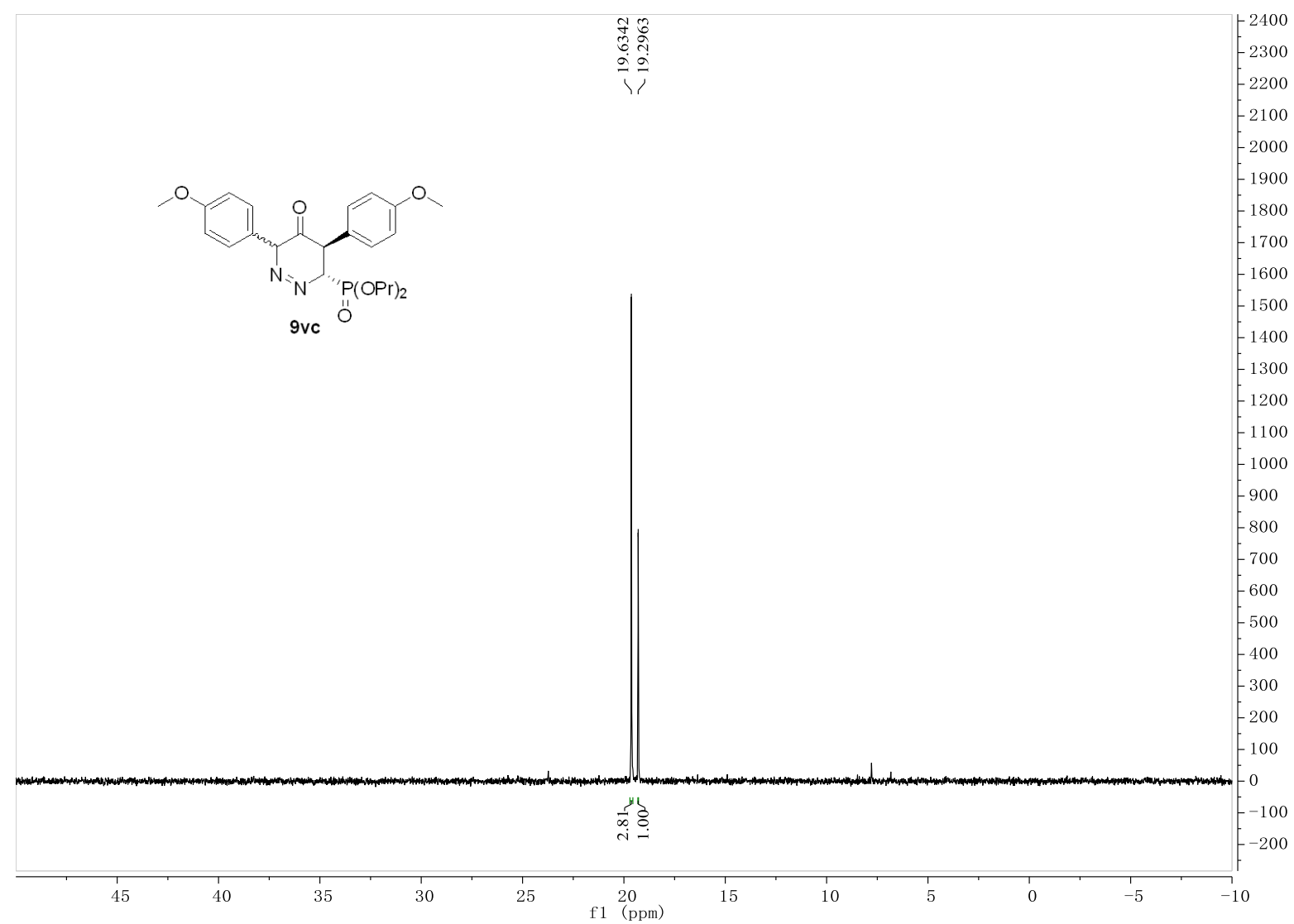

10. Deuterium experiments and controlling experiments

\subsection{Preparation of deuteriated $\alpha$-bromoketone}

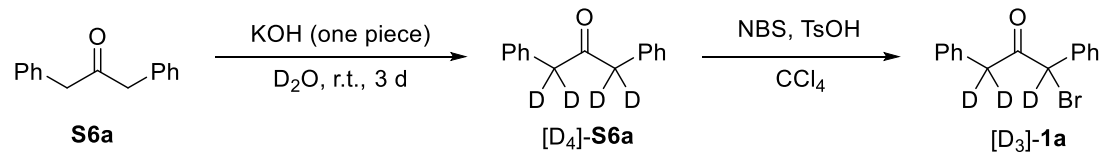

\section{Preparation of 1,3-bisphenyl-2-propanone- $d_{4}$}

Deuterium oxide $(10 \mathrm{~mL}, 500 \mathrm{mmol})$ was placed in a dried $25 \mathrm{~mL}$ round-bottom flask containing a magnetic stir bar. A pellet of $\mathrm{KOH}$ was added and the solution was stirred for 10 min. 1, 3-Bisphenyl-2-propanone $(1.28 \mathrm{~mL}, 6.3 \mathrm{mmol})$ was added and the solution was vigorously stirred 3 days at room temperature. The solution was extracted with $\mathrm{Et}_{2} \mathrm{O}$, dried with $\mathrm{Na}_{2} \mathrm{SO}_{4}$, and the solvent was removed under vacuum to yield $1.32 \mathrm{~g}(98 \%)$ of deuterated 1,3-diphenylpropan-2-one- $d_{4}\left(\left[\mathrm{D}_{4}\right]-\mathrm{S6a}, 97.5 \% \mathrm{D}\right.$ by $\left.{ }^{1} \mathrm{H} \mathrm{NMR}\right) .{ }^{1} \mathrm{H}$ NMR $\left(600 \mathrm{MHz}, \mathrm{CDCl}_{3}\right)$ $\delta 7.35(\mathrm{t}, J=7.3 \mathrm{~Hz}, 2 \mathrm{H}), 7.32-7.28(\mathrm{~m}, 1 \mathrm{H}), 7.19(\mathrm{~d}, J=7.4 \mathrm{~Hz}, 2 \mathrm{H}) ;{ }^{13} \mathrm{C}$ NMR $(151 \mathrm{MHz}$, $\left.\mathrm{CDCl}_{3}\right) \delta 205.6,133.9,129.4,128.6,127.0,48.8-48.5(\mathrm{~m}), 48.3\left(\mathrm{p}, J_{\mathrm{CD}}=19.4 \mathrm{~Hz}\right) .{ }^{1} \mathrm{H} \mathrm{NMR}$ $\left(600 \mathrm{MHz}, \mathrm{CDCl}_{3}\right) \delta 7.4-7.3(\mathrm{~m}, 2 \mathrm{H}), 7.3-7.3(\mathrm{~m}, 3 \mathrm{H}), 7.3-7.2(\mathrm{~m}, 3 \mathrm{H}), 7.1(\mathrm{~d}, J=7.4 \mathrm{~Hz}$, $2 \mathrm{H})$. 


\section{Preparation of 1-bromo-1,3-diphenylpropan-2-one- $d_{3}$}

$\mathrm{TsOH} \cdot \mathrm{H}_{2} \mathrm{O}$ (0.1 equiv.) was added to a solution of $\left[\mathrm{D}_{4}\right]-$ S6a (1.0 equiv.) and NBS (1.2 equiv.) in $\mathrm{CCl}_{4}(1 \mathrm{M})$ at room temperature under an argon atmosphere. The mixture was stirred at $30{ }^{\circ} \mathrm{C}$ until the starting material was disappeared. The reaction mixture was purified directly by column chromatography $\left(\mathrm{PE} / \mathrm{Et}_{2} \mathrm{O}=20 / 1-10 / 1\right)$ to afford the deuteriated $\alpha$-bromoketone $\left[\mathrm{D}_{3}\right]-\mathbf{1 a}$ (98\% yield, $>95 \%$ D by ${ }^{1} \mathrm{H}$ NMR). Light pink solid; mp $41.2-43.0{ }^{\circ} \mathrm{C}$; ${ }^{1} \mathrm{H}$ NMR (600 MHz, $\left.\mathrm{CDCl}_{3}\right) \delta$ 7.37-7.35 (m, 2H), 7.34-7.31 (m, 3H), 7.29-7.22 (m, 3H), $7.08(\mathrm{~d}, J=7.4 \mathrm{~Hz}, 2 \mathrm{H}) ;{ }^{13} \mathrm{C} \mathrm{NMR}\left(150 \mathrm{MHz}, \mathrm{CDCl}_{3}\right) \delta 198.7,134.9,133.3,129.5,129.3$, 129.1, 129.0, 128.8, 127.4, 54.5 (dd, $J=50.4,27.8 \mathrm{~Hz}), 46.2-45.0$ (m); HRMS (ESI): $m / z$ calcd. for $\mathrm{C}_{15} \mathrm{H}_{10} \mathrm{BrD}_{3} \mathrm{NaO}$ [M-Na] $]^{+}$314.0230, found: 314.0230 .

\subsection{Kinetic isotope effect study}

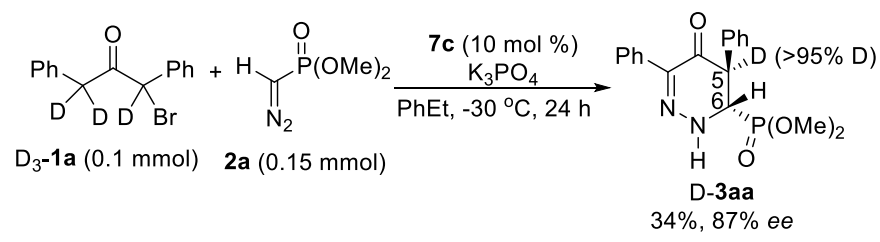

2a $(0.15 \mathrm{mmol})$ was added to a mixture of catalyst $7 \mathbf{c}(0.01 \mathrm{mmol}), \mathrm{K}_{3} \mathrm{PO}_{4}(0.3 \mathrm{mmol})$, $\left[\mathrm{D}_{3}\right]-1 \mathrm{a}(0.1 \mathrm{mmol})$ in anhydrous ethylbenzene $(1.0 \mathrm{~mL})$ at $-30{ }^{\circ} \mathrm{C}$. The mixture was then stirred vigorously for $24 \mathrm{~h}$. The reaction was treated with acetic acid $(30 \mu \mathrm{L})$ and stopped with remaining starting material. Purification was performed rapidly by a short column chromatography on silica gel using petroleum ether/acetone $(3 / 2 \mathrm{v} / \mathrm{v})$ as the eluents to afford the deuteriated product D-3aa.

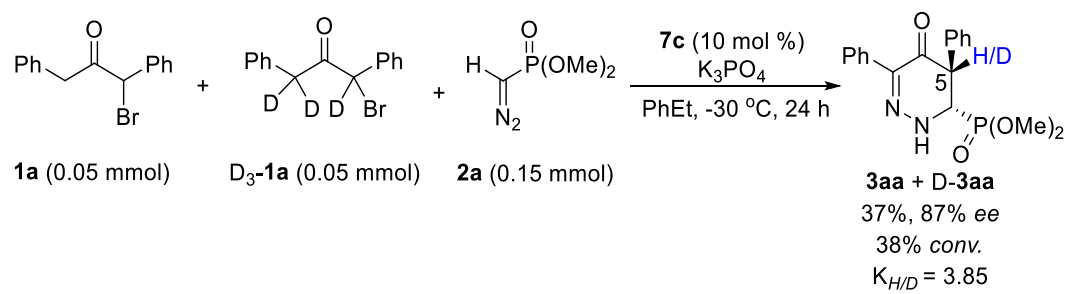

2a $(0.15 \mathrm{mmol})$ was added to a mixture of catalyst $\mathbf{7 c}(0.01 \mathrm{mmol}), \mathrm{K}_{3} \mathrm{PO}_{4}(0.3 \mathrm{mmol}), \mathbf{1 a}$ $(0.05 \mathrm{mmol})$ and $\left[\mathrm{D}_{3}\right]-\mathbf{1 a}(0.05 \mathrm{mmol})$ in anhydrous ethylbenzene $(1.0 \mathrm{~mL})$ at $-30{ }^{\circ} \mathrm{C}$. The mixture was then stirred vigorously for $24 \mathrm{~h}$. The reaction was treated with acetic acid $(30 \mu \mathrm{L})$ and stopped with remaining starting material. Purification was performed rapidly by a short column chromatography on silica gel using petroleum ether/acetone $(3 / 2 \mathrm{v} / \mathrm{v})$ as the eluents to afford the product as mixture of 3aa and D-3aa in 37\% isolated yield (38\% conversion for $\alpha$-bromoketone, calculated by total recovery rate of $\mathbf{1 a}$ and $\left.\left[\mathrm{D}_{3}\right]-\mathbf{1 a}\right)$. The $\mathrm{H} / \mathrm{D}$ rate at $\mathrm{C} 5$ position of the product was determined by ${ }^{1} \mathrm{H} \mathrm{NMR}\left(600 \mathrm{MHz}, \mathrm{CDCl}_{3}\right)$. 


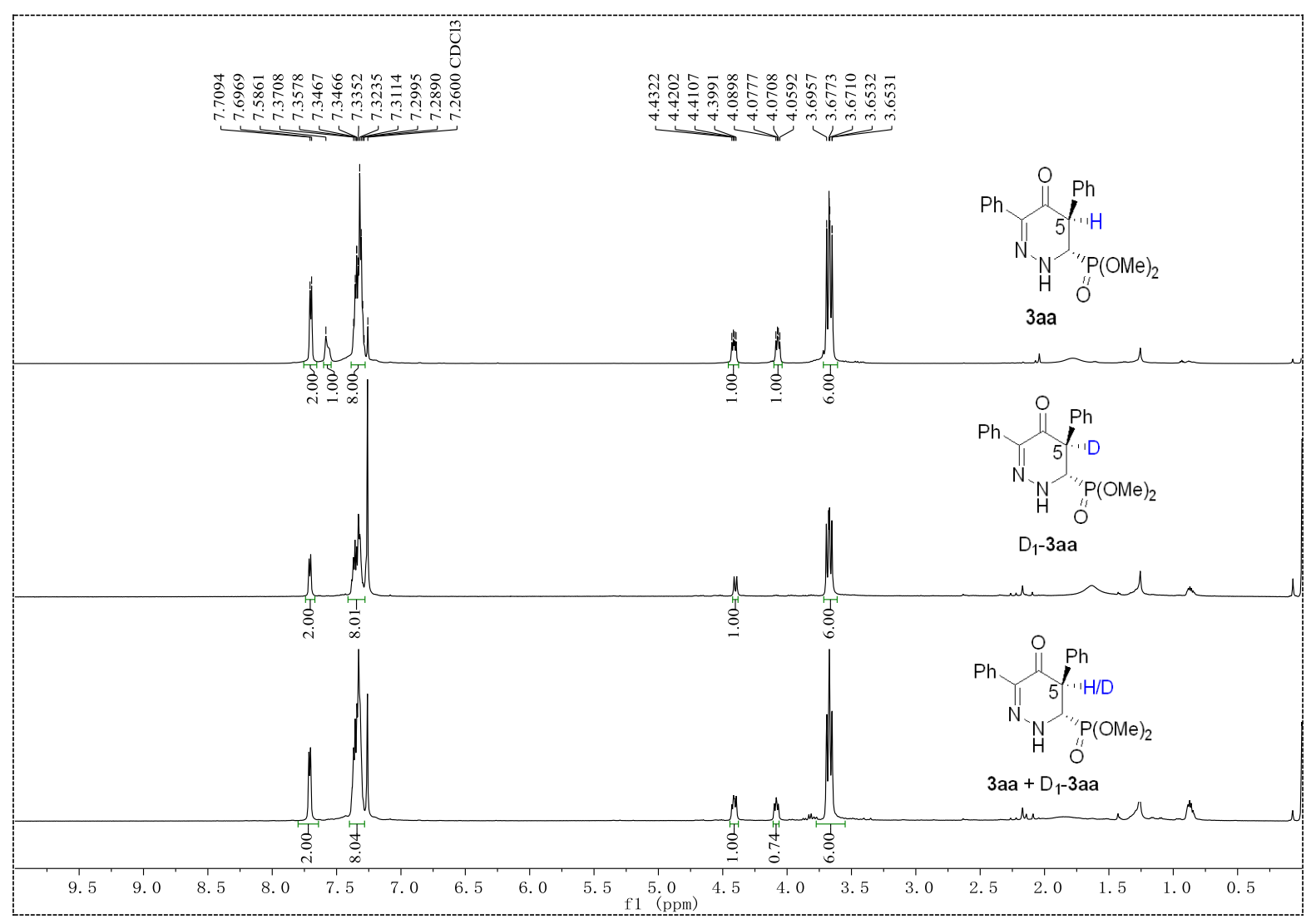

\subsection{Controlling experiments}

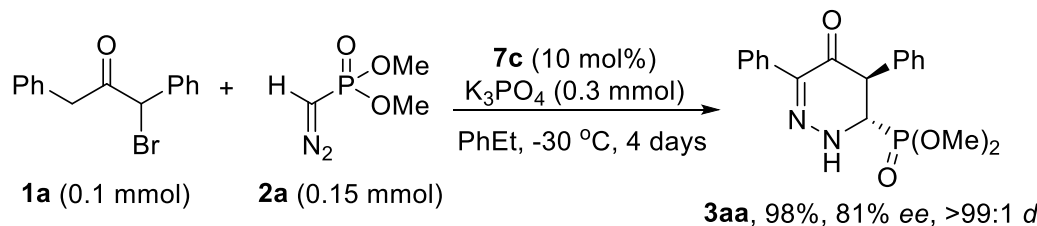

$$
\underset{(0.1 \mathrm{mmol})}{\mathbf{1 a}}+\underset{(0.15 \mathrm{mmol})}{\mathbf{2 a}} \stackrel{\mathrm{K}_{3} \mathrm{PO}_{4}(0.3 \mathrm{mmol})}{\mathrm{PhEt},-30^{\circ} \mathrm{C}, 4 \text { days }} \text { N.R. }
$$

$\underset{(0.01 \mathrm{mmol})}{7 \mathrm{c}} \stackrel{\mathrm{K}_{3} \mathrm{PO}_{4}(0.3 \mathrm{mmol})}{\stackrel{\mathrm{PhEt}, 30^{\circ} \mathrm{C}, 3 \mathrm{~h}}{\longrightarrow}} \stackrel{\begin{array}{l}1 \mathbf{a}(0.1 \mathrm{mmol}) \\ \mathbf{2 a}(0.15 \mathrm{mmol})\end{array}}{-30^{\circ} \mathrm{C}, 4 \text { days }} \begin{gathered}\text { 3aa } \\ 65 \%, 74 \% \text { ee, }>99: 1 \mathrm{dr}\end{gathered}$




\section{References}

[1] Coric, I.; List, B., Asymmetric Spiroacetalization Catalysed by Confined Bronsted Acids. Nature 2012, 483, 315.

[2] Chen, Y.-Y.; Jiang, Y.-J.; Fan, Y.-S.; Sha, D.; Wang, Q.; Zhang, G.; Zheng, L.; Zhang, S., Double axially chiral bisphosphorylimides as novel Brønsted acids in asymmetric three-component Mannich reaction. Tetrahedron: Asymmetry 2012, 23, 904.

[3] Bhandari, S.; Ray, S., A Novel Synthesis of Bisbenzyl Ketones by DCC Induced Condensation of Phenylacetic Acid. Synth. Commun. 1998, 28, 765.

[4] Leusen, D. V.; Leusen, A. M. V. Synthetic Uses of Tosylmethyl Isocyanide (TosMIC). In Organic Reactions, Vol. 57 (Eds.: L. E. Overman), John Wiley \& Sons, 2001, pp. 417.

[5] a) Yodov, J. S.; Ready, P. S.; Joshi, B. V., A Convenient Reduction of Alkylated Tosylmethyl Isocyanides: Applications for the Synthesis of Natural Products. Tetrahedron 1988, 44, 7243. b) Wang, L.; Studer, A., 1-Trifluoromethylisoquinolines from a-Benzylated Tosylmethyl Isocyanide Derivatives in a Modular Approach. Org. Lett. 2017, 19,5701 . 


\section{The spectra of NMR}

$600 \mathrm{MHz}, \mathrm{CDCl}_{3},{ }^{1} \mathrm{H} \mathrm{NMR}$

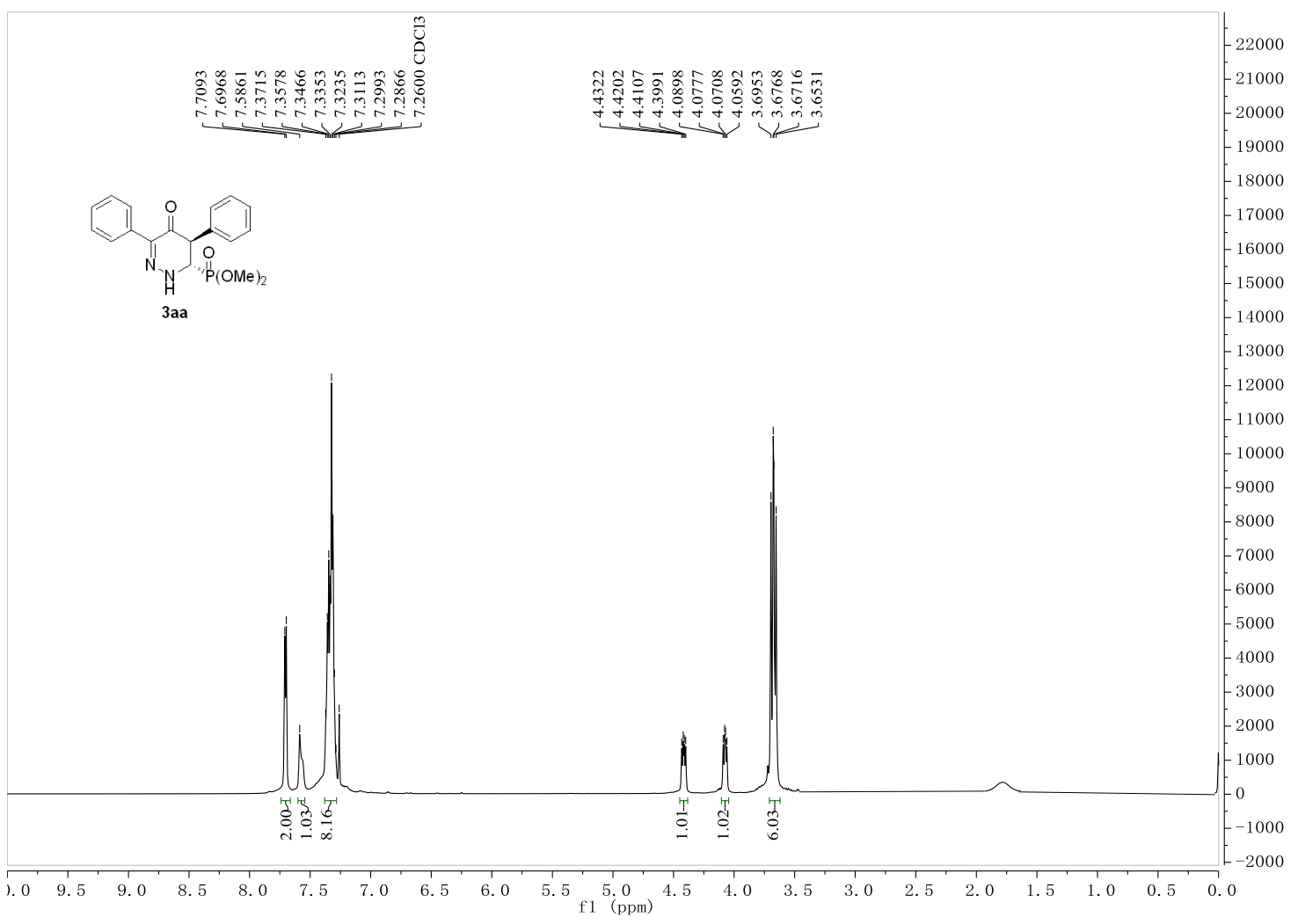

$151 \mathrm{MHz}, \mathrm{CDCl}_{3},{ }^{13} \mathrm{C} \mathrm{NMR}$

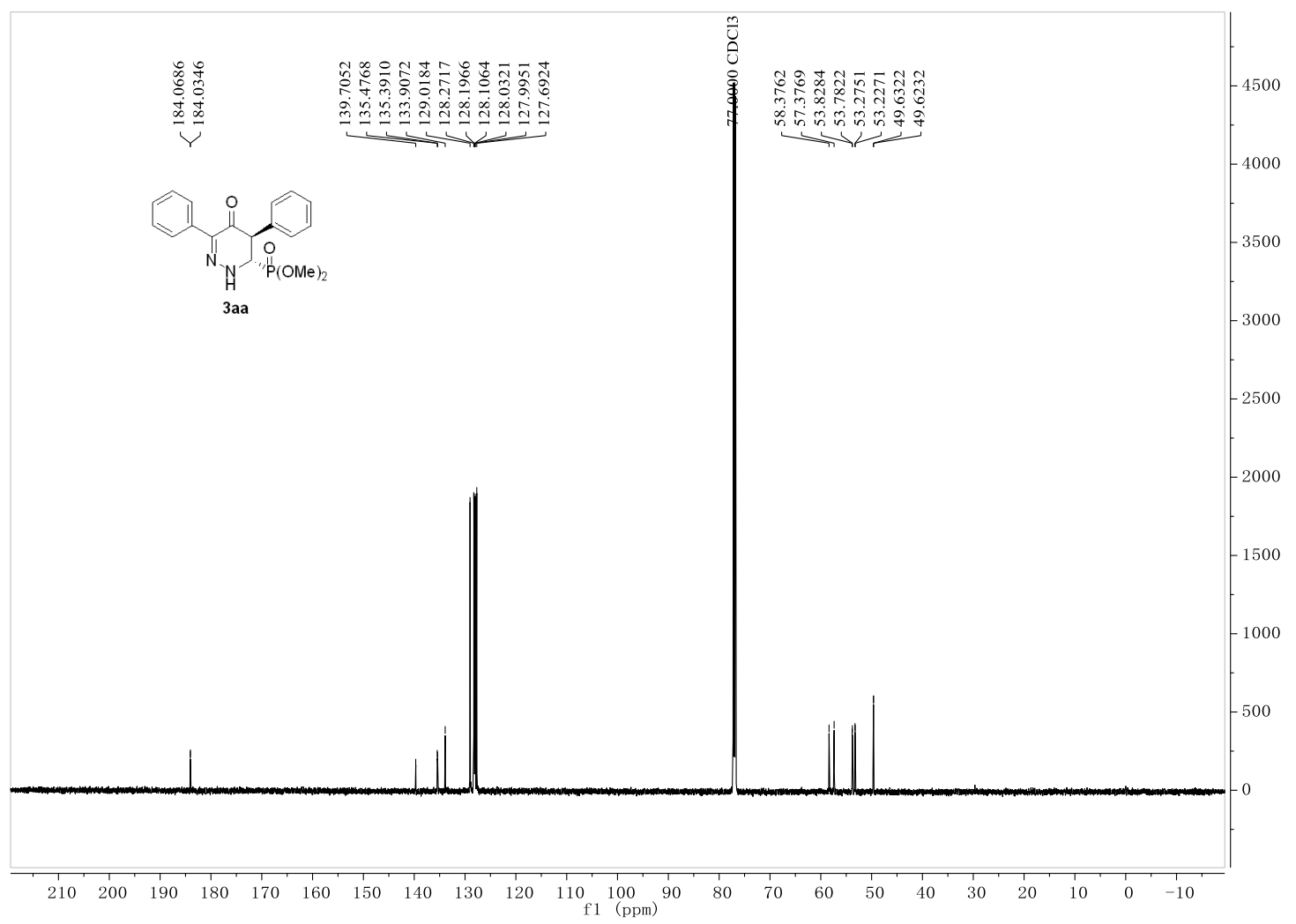




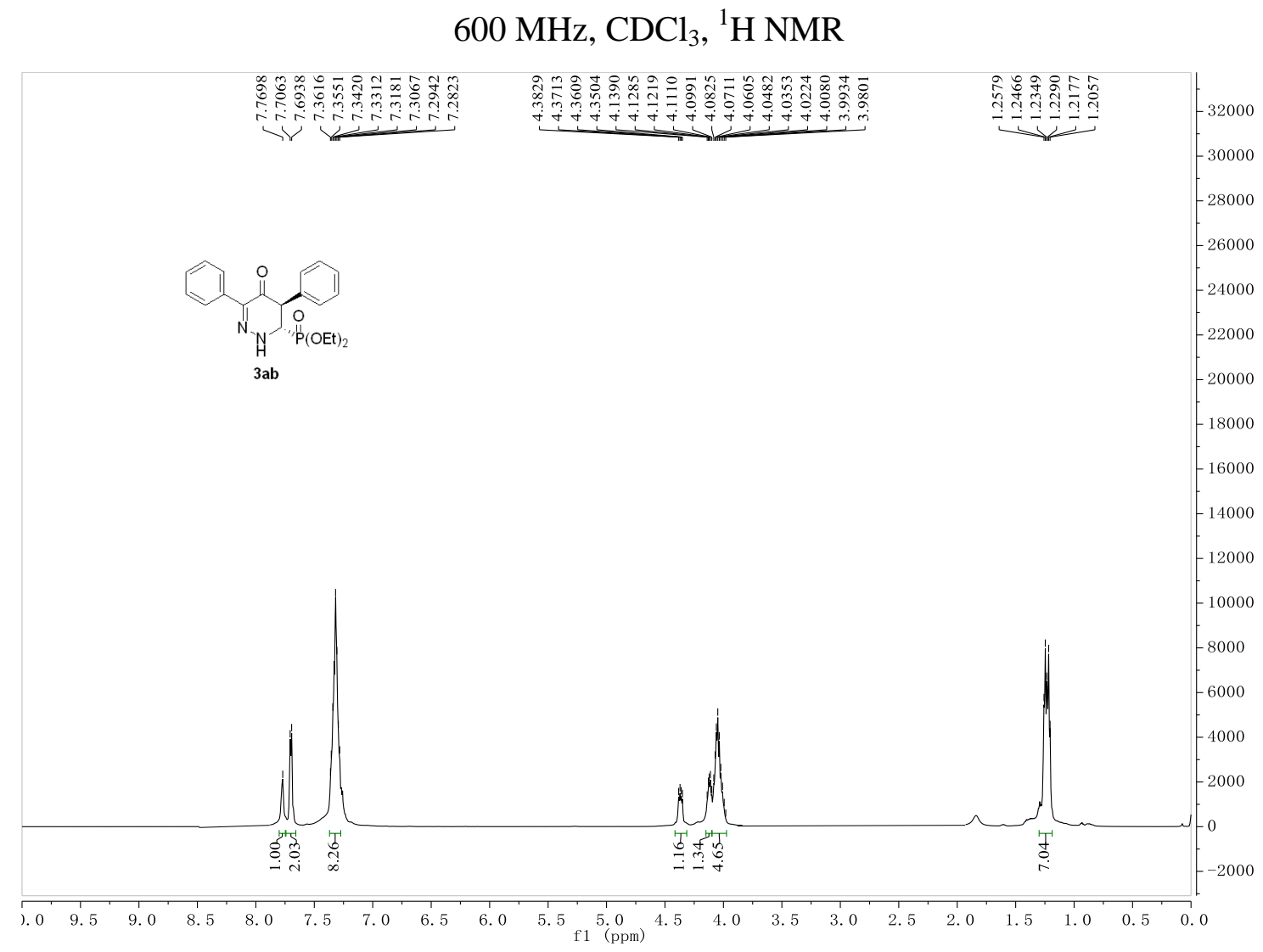

$151 \mathrm{MHz}, \mathrm{CDCl}_{3},{ }^{13} \mathrm{C} \mathrm{NMR}$

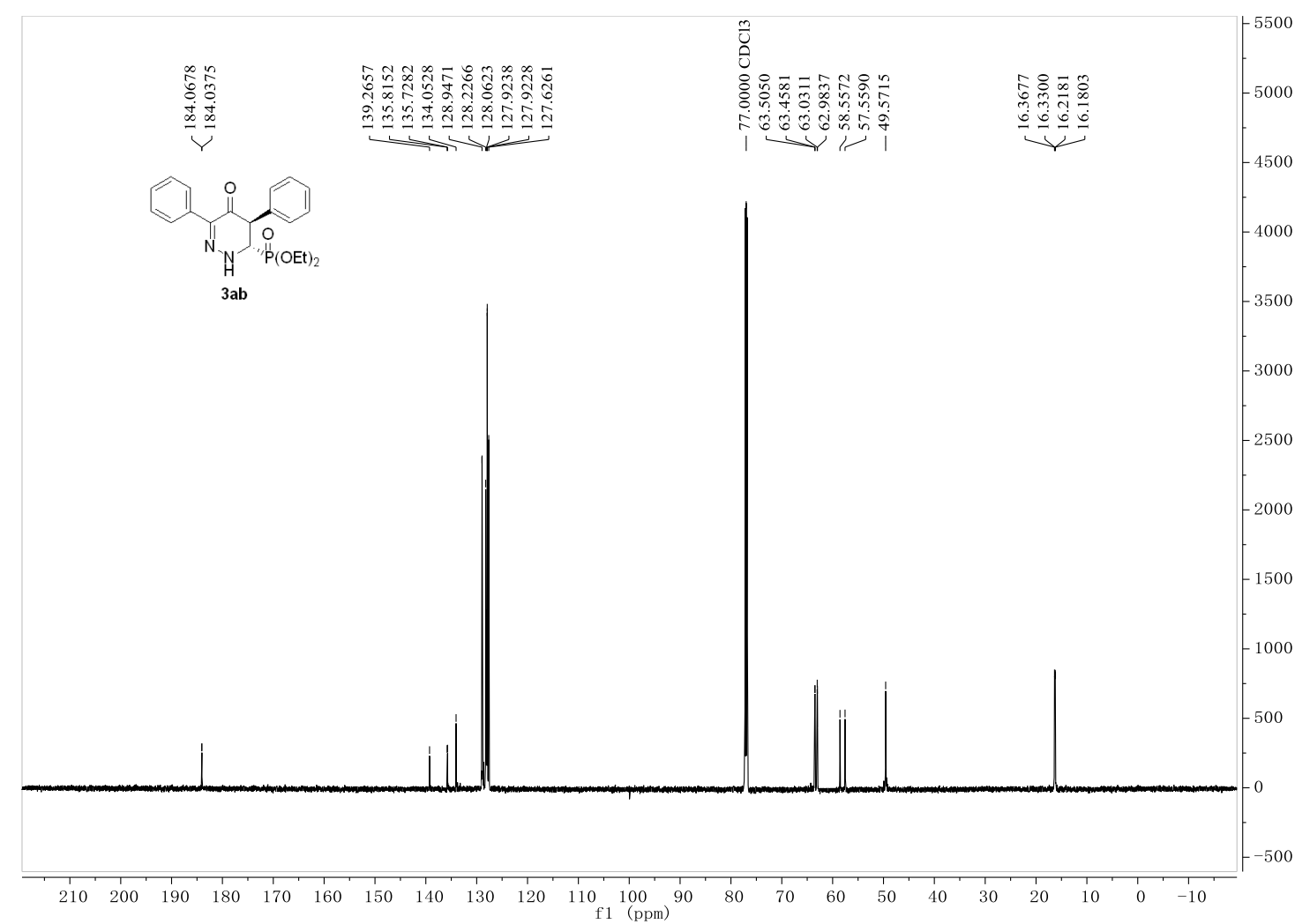




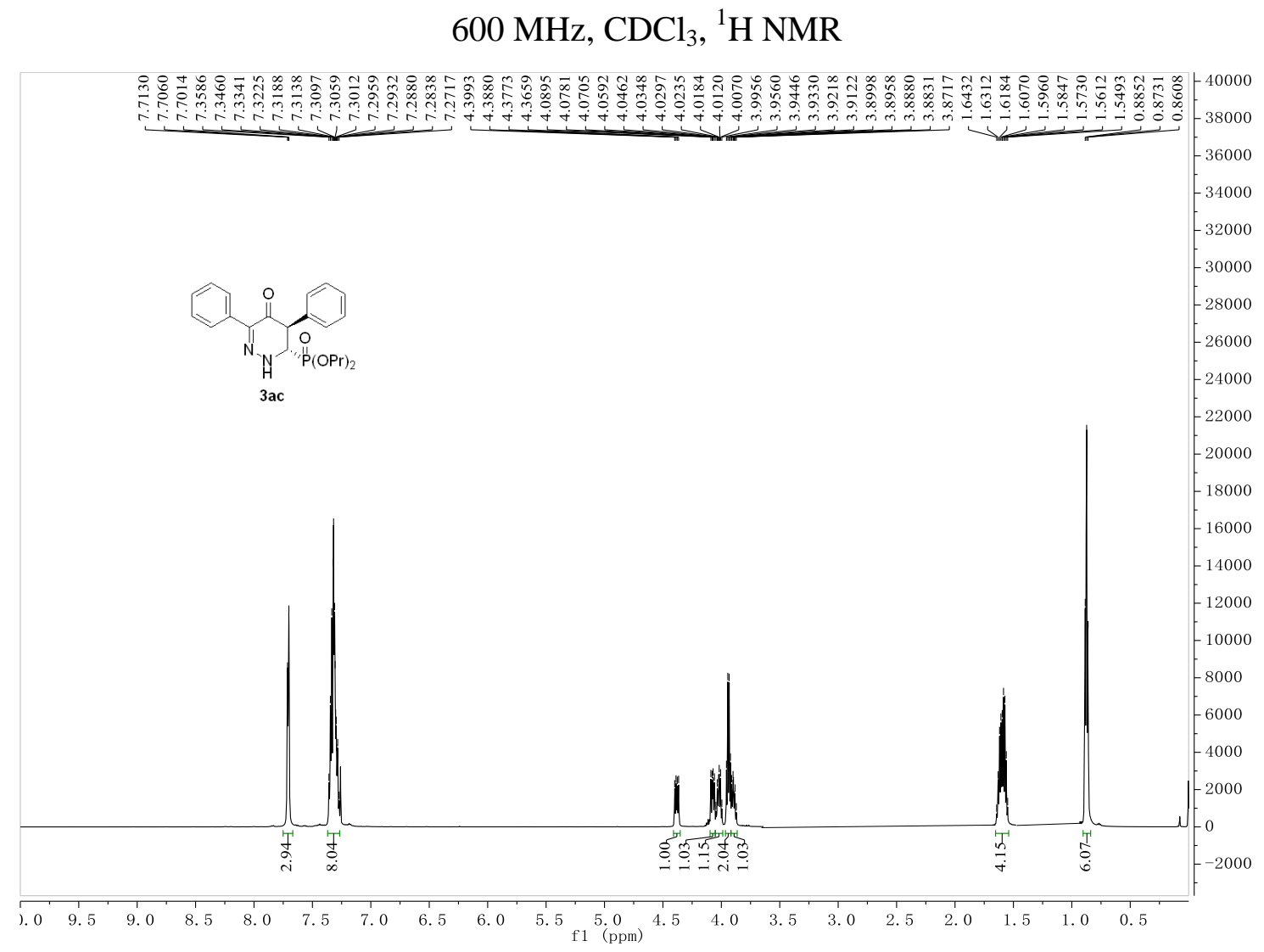

$151 \mathrm{MHz}, \mathrm{CDCl}_{3},{ }^{13} \mathrm{C} \mathrm{NMR}$

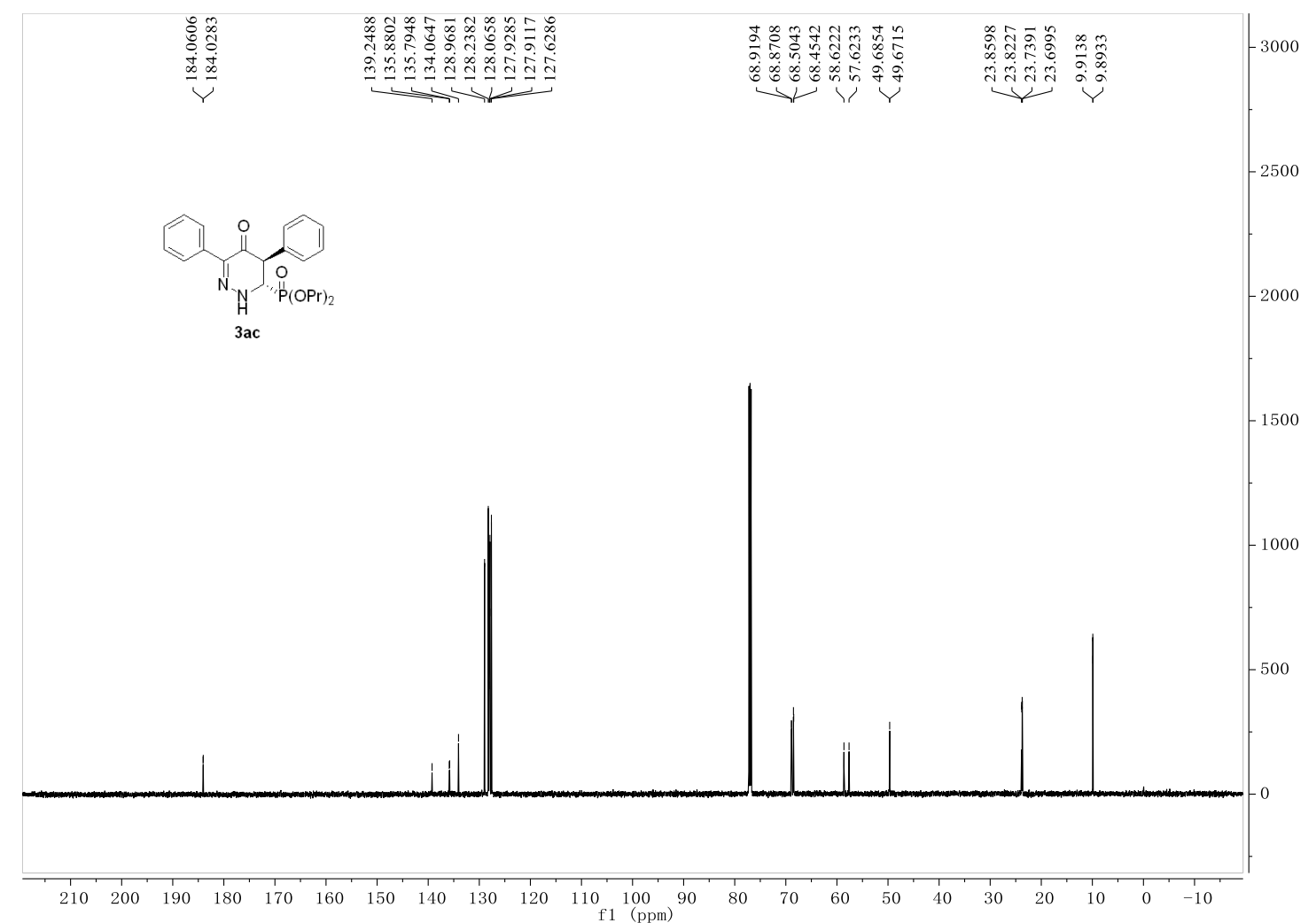


$600 \mathrm{MHz}, \mathrm{CDCl}_{3},{ }^{1} \mathrm{H} \mathrm{NMR}$

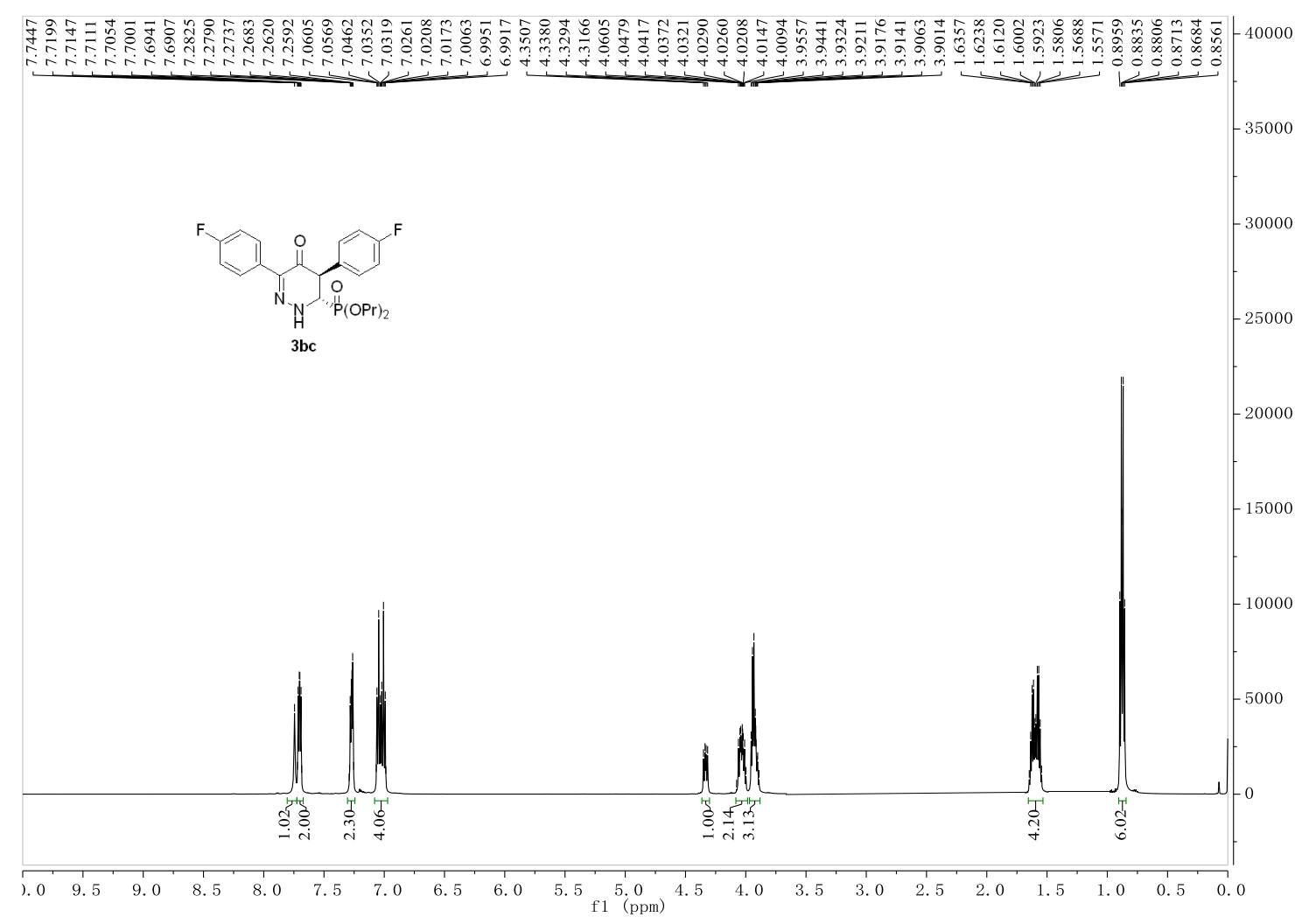

$151 \mathrm{MHz}, \mathrm{CDCl}_{3},{ }^{13} \mathrm{C} \mathrm{NMR}$

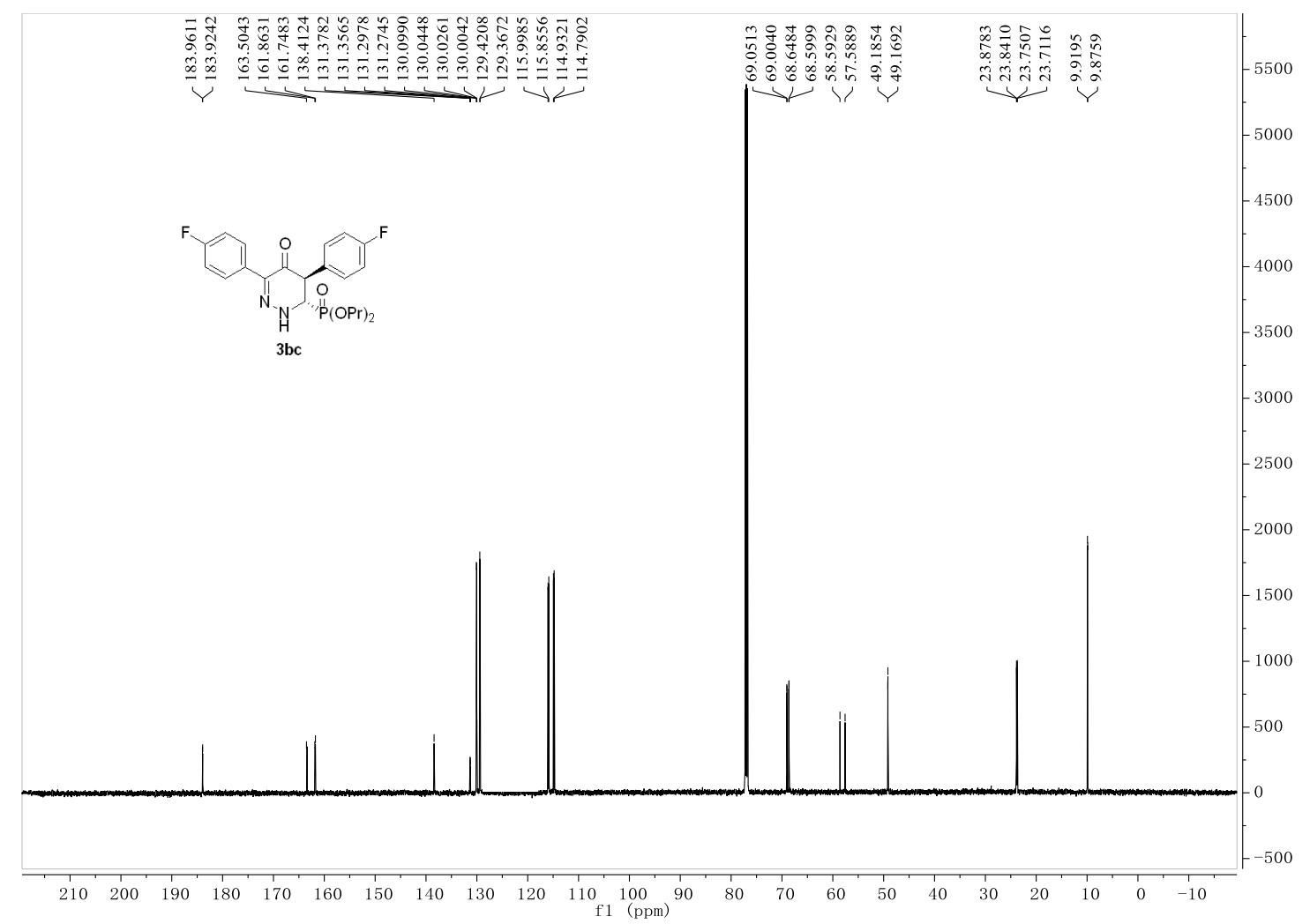




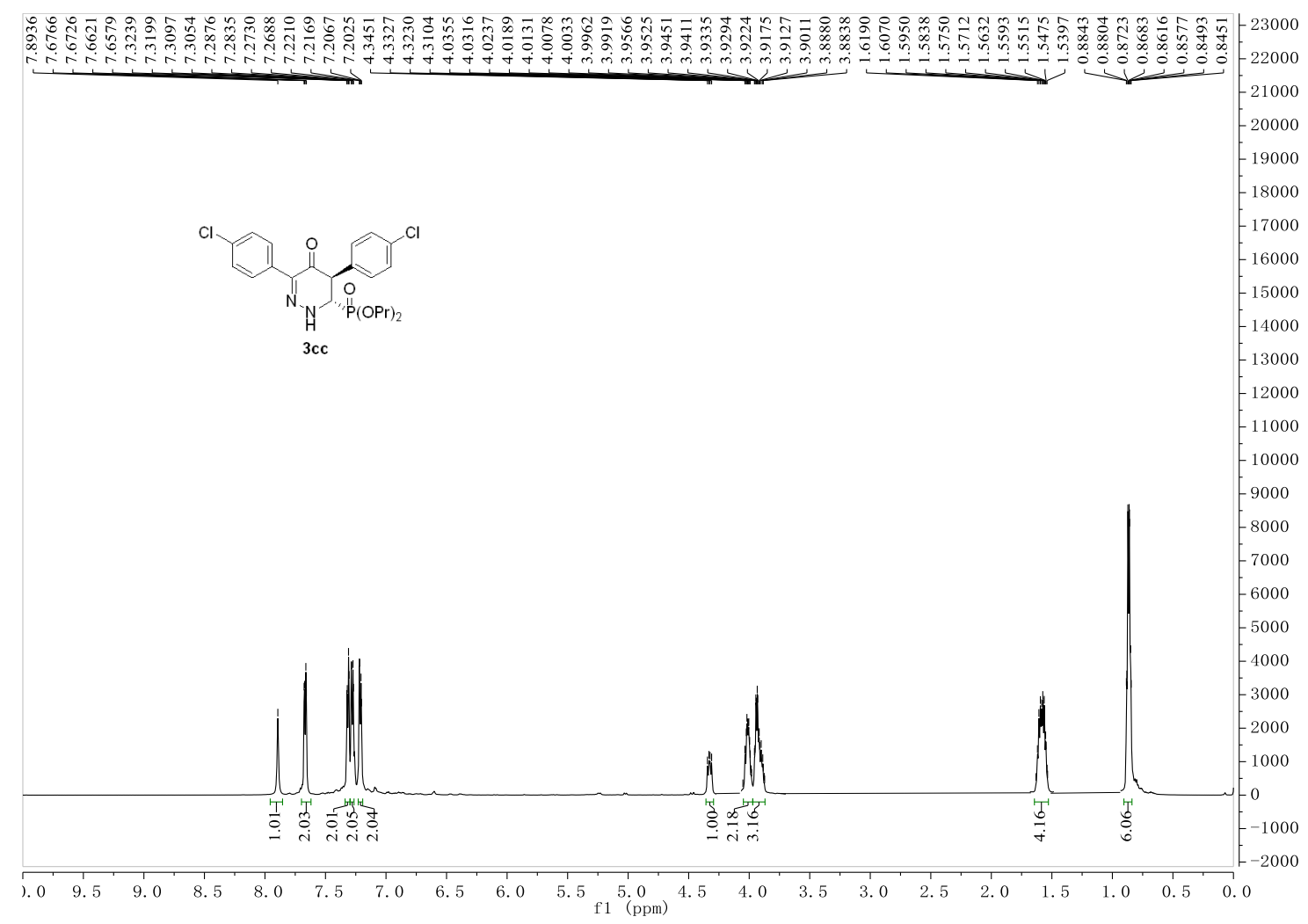

$151 \mathrm{MHz}, \mathrm{CDCl}_{3},{ }^{13} \mathrm{C} \mathrm{NMR}$

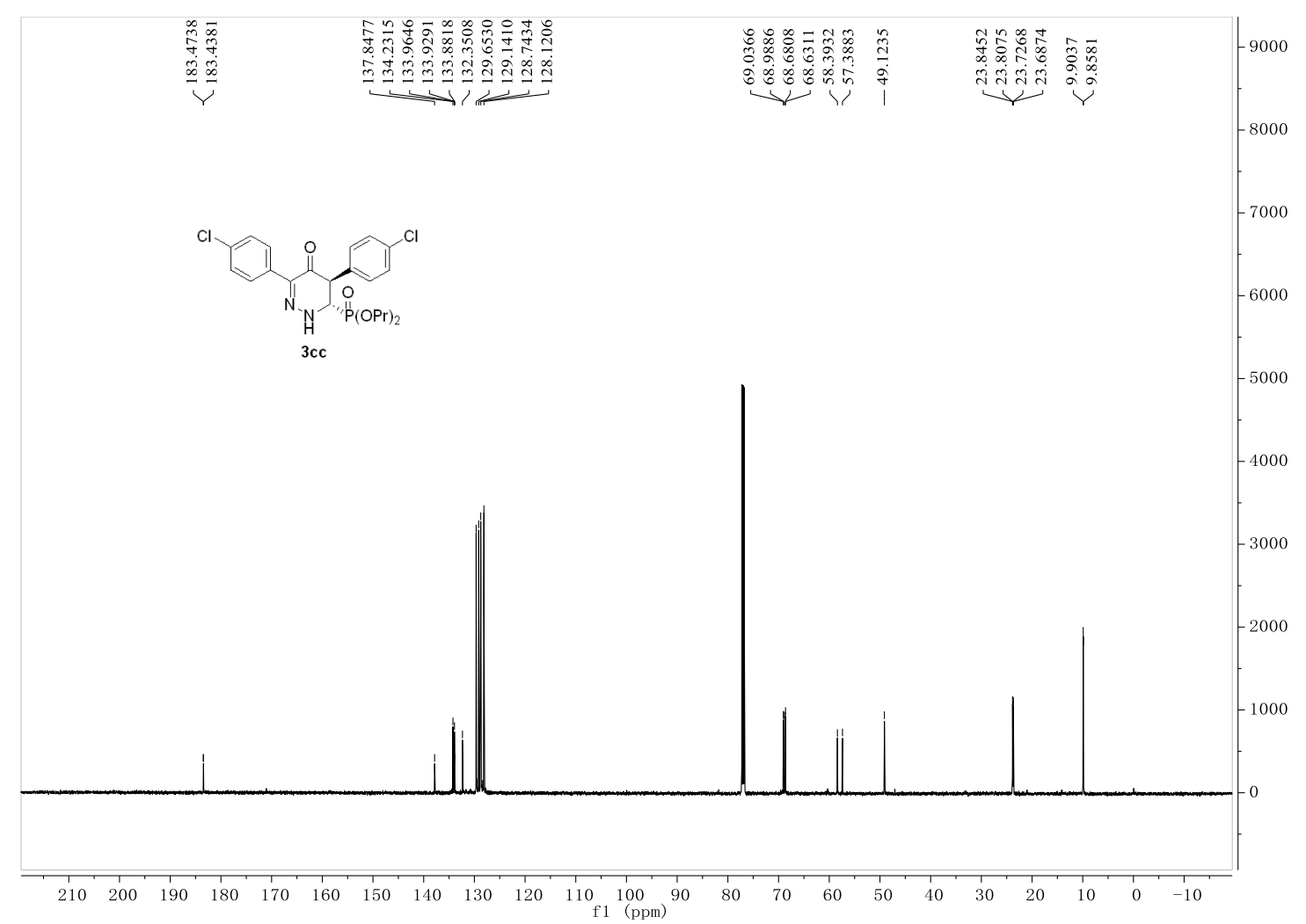


$600 \mathrm{MHz}, \mathrm{CDCl}_{3},{ }^{1} \mathrm{H} \mathrm{NMR}$

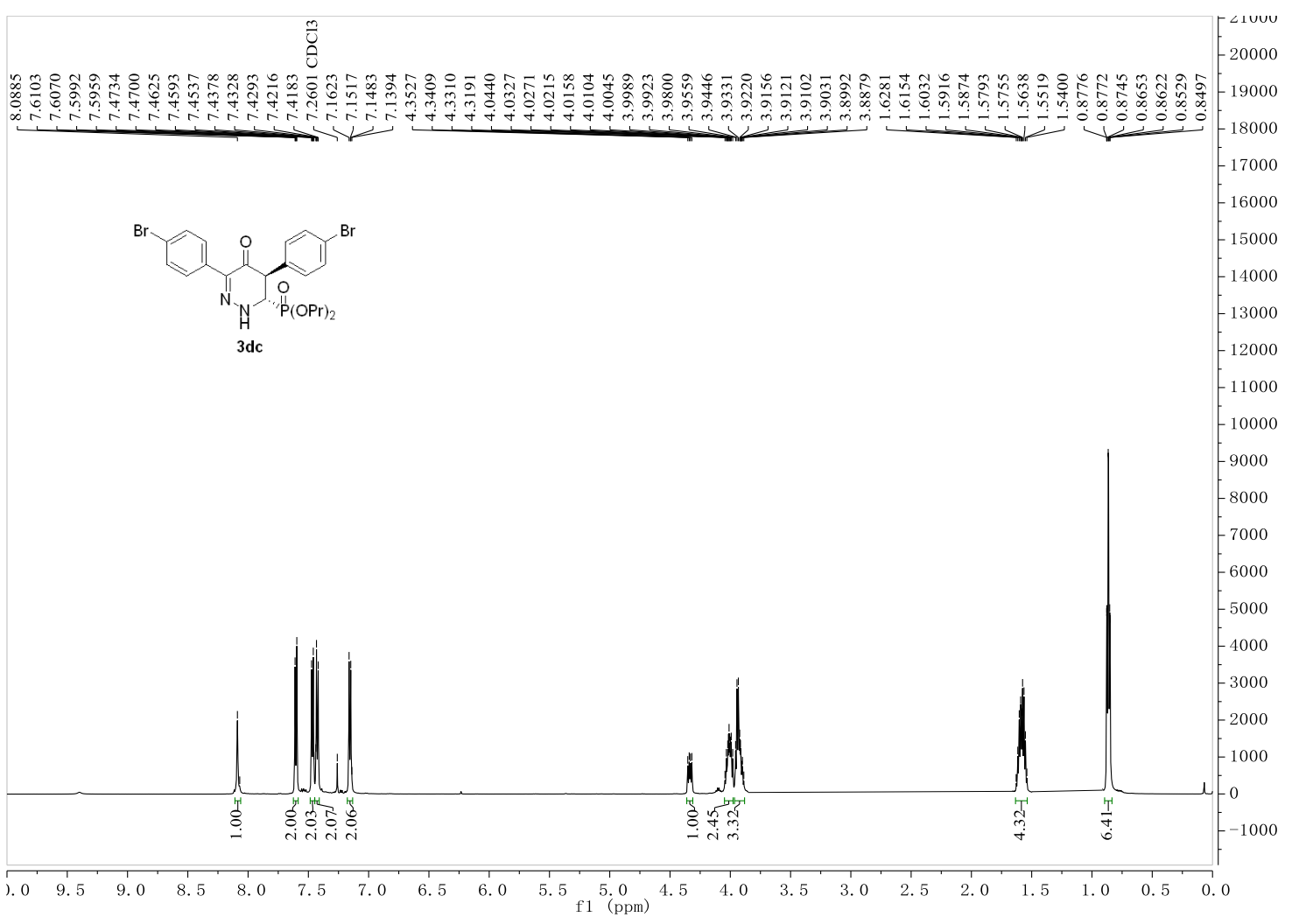

$151 \mathrm{MHz}, \mathrm{CDCl}_{3},{ }^{13} \mathrm{C} \mathrm{NMR}$

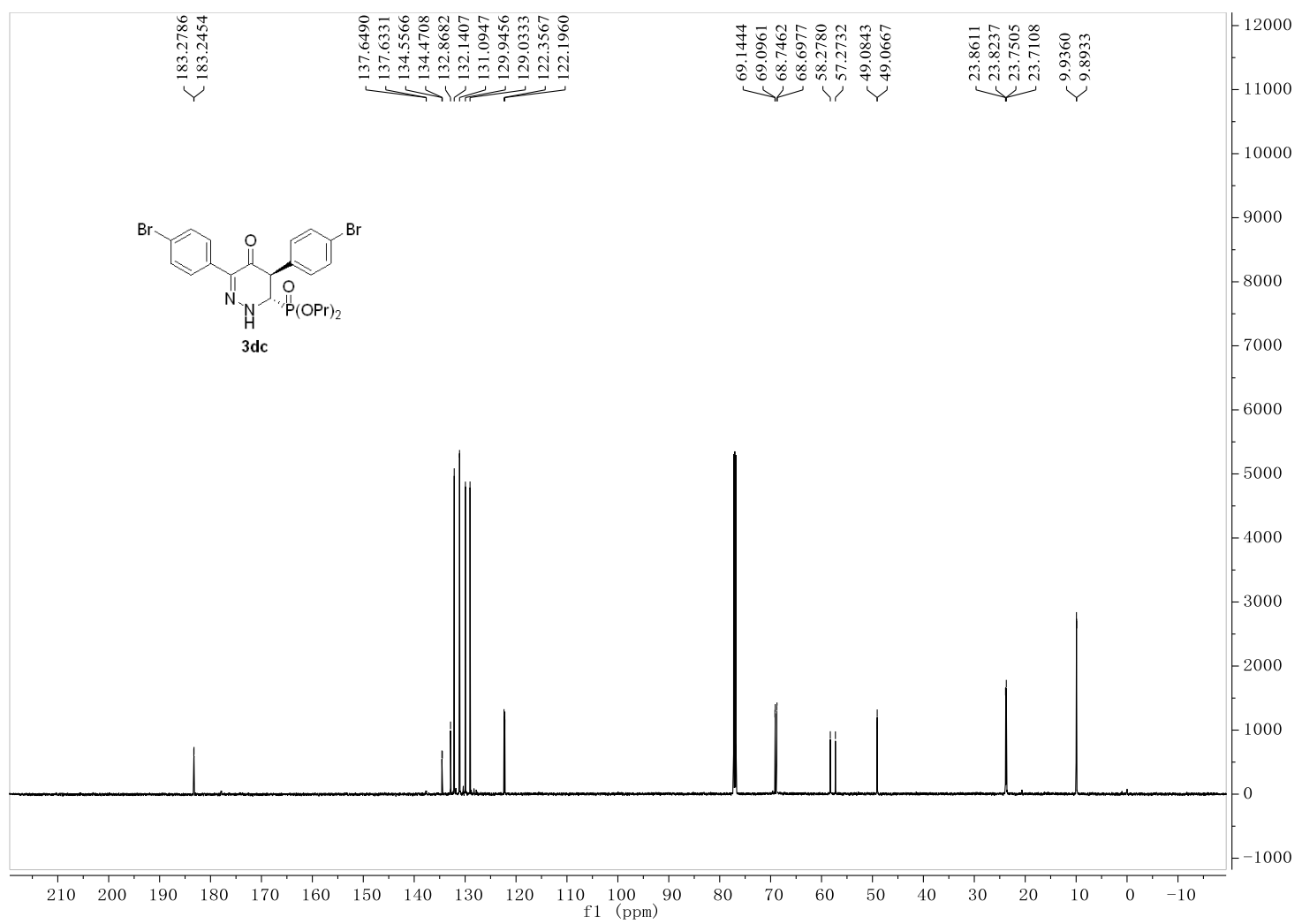




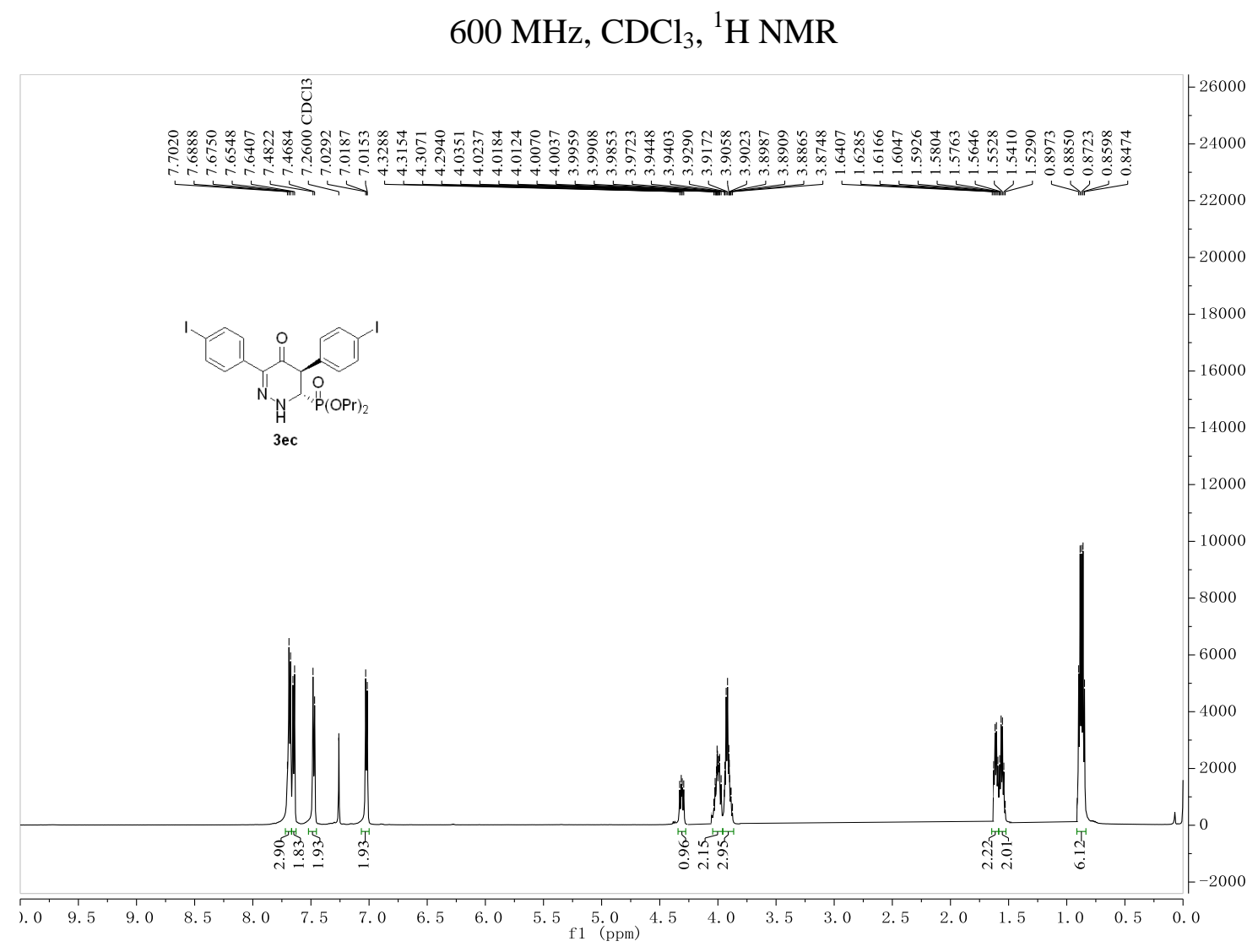

$151 \mathrm{MHz}, \mathrm{CDCl}_{3},{ }^{13} \mathrm{C} \mathrm{NMR}$

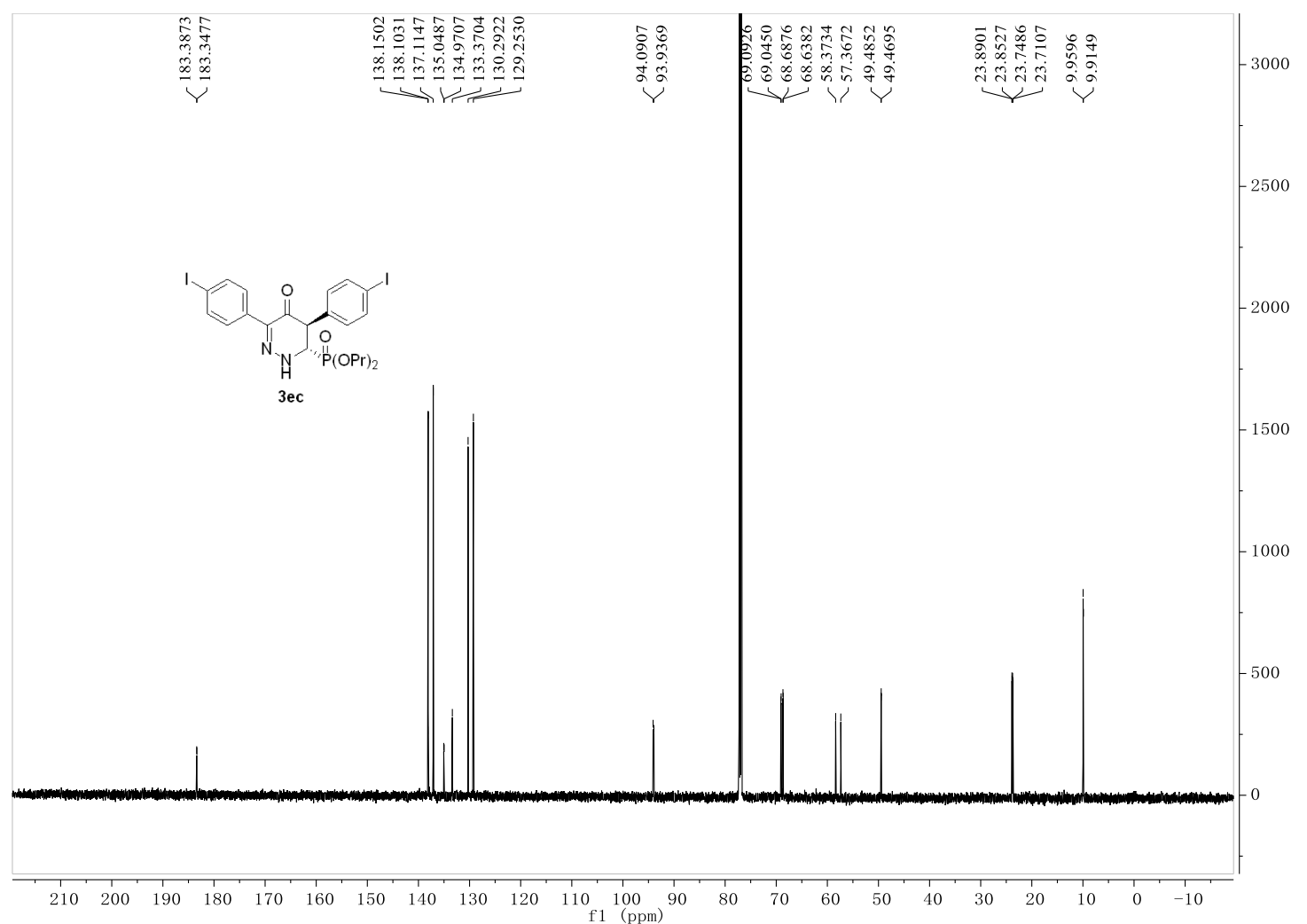




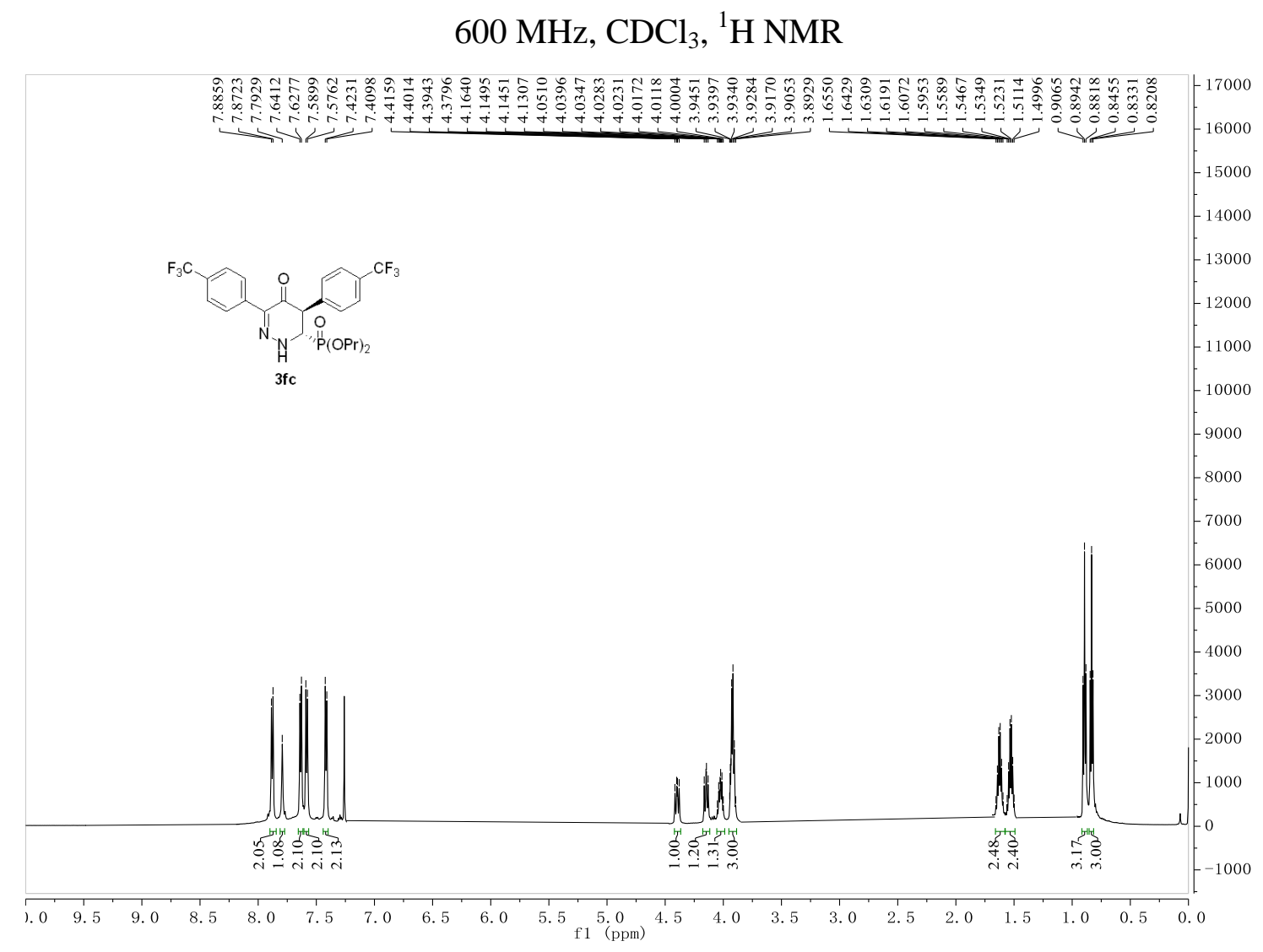

$151 \mathrm{MHz}, \mathrm{CDCl}_{3},{ }^{13} \mathrm{C} \mathrm{NMR}$

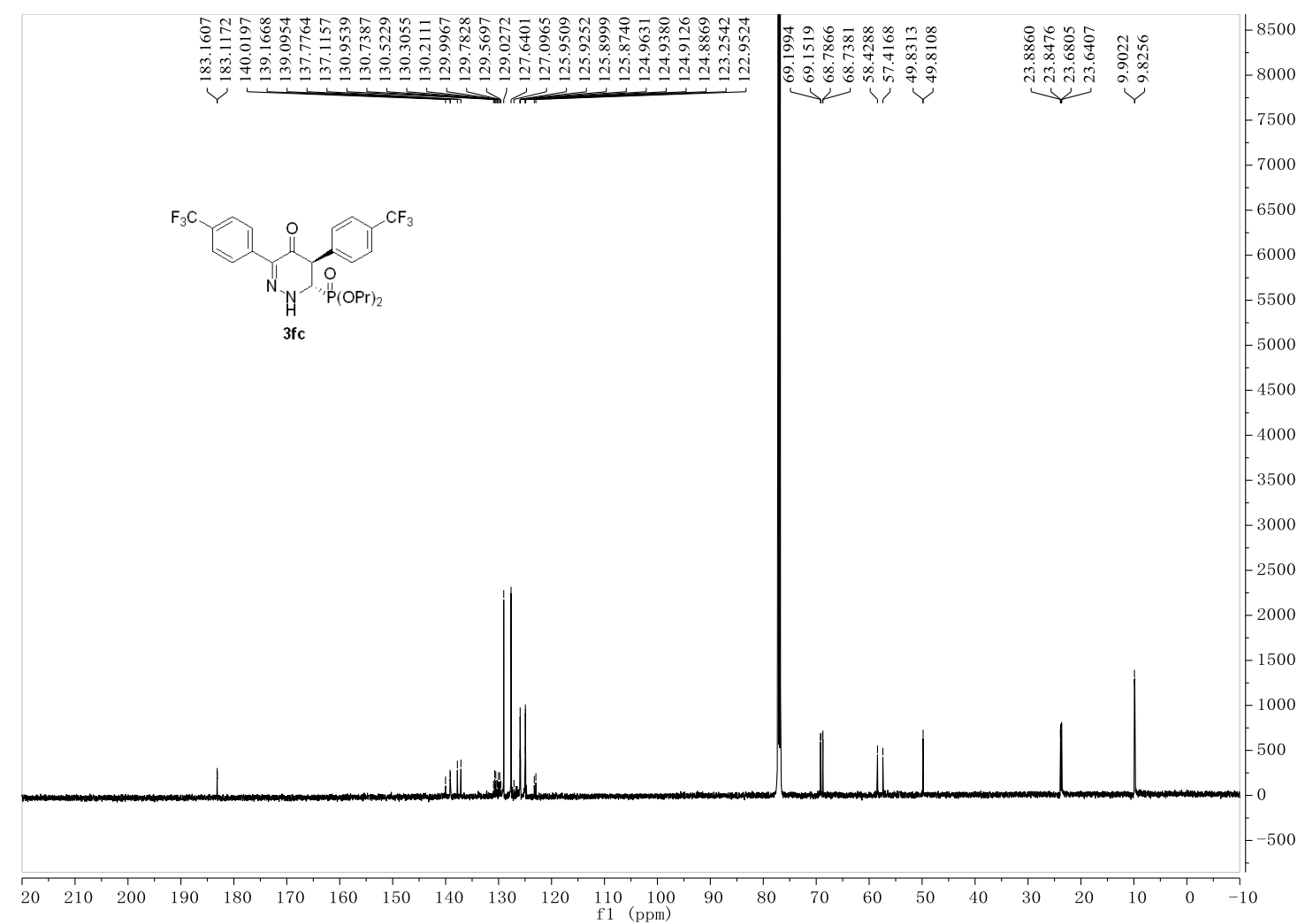




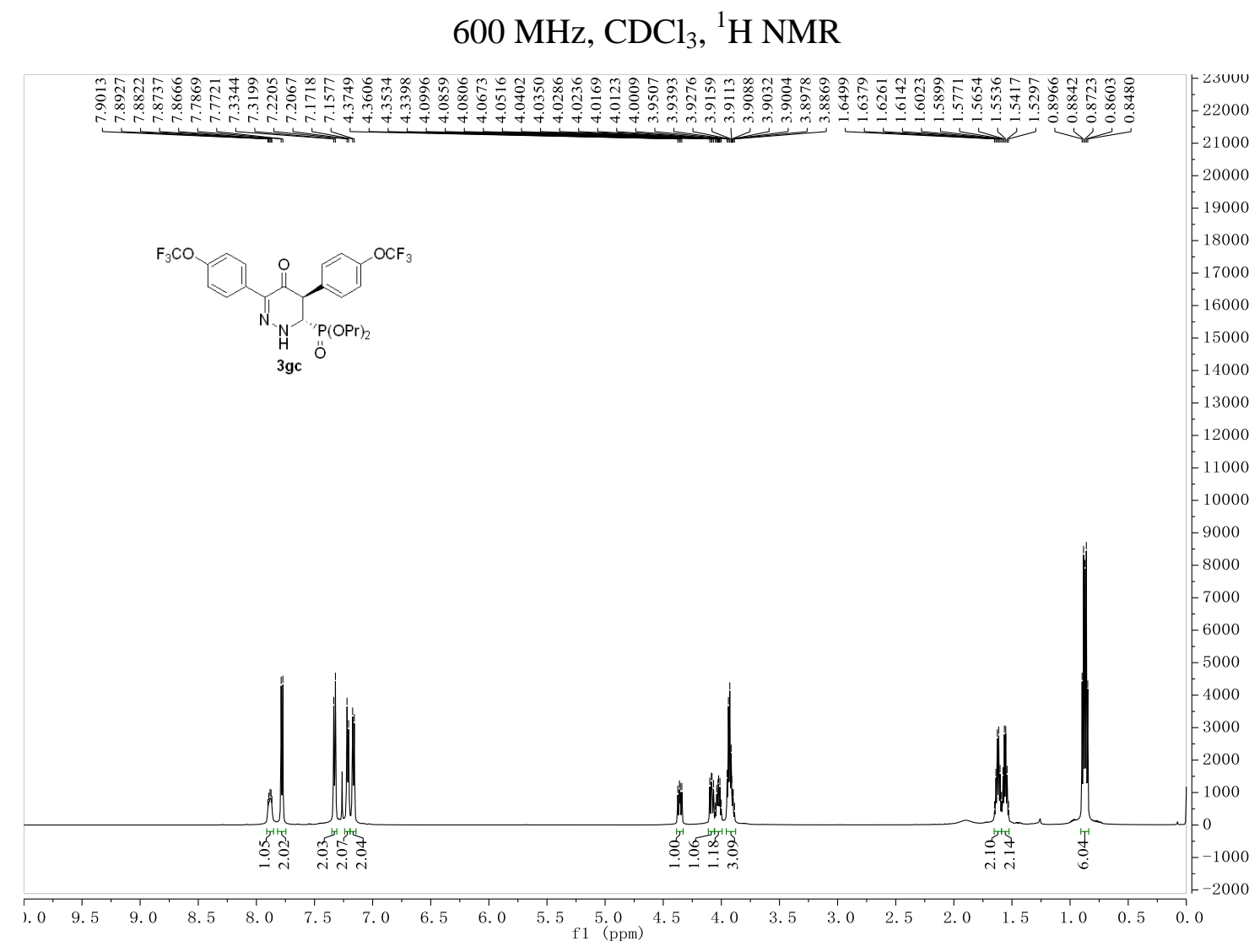

$151 \mathrm{MHz}, \mathrm{CDCl}_{3},{ }^{13} \mathrm{C} \mathrm{NMR}$

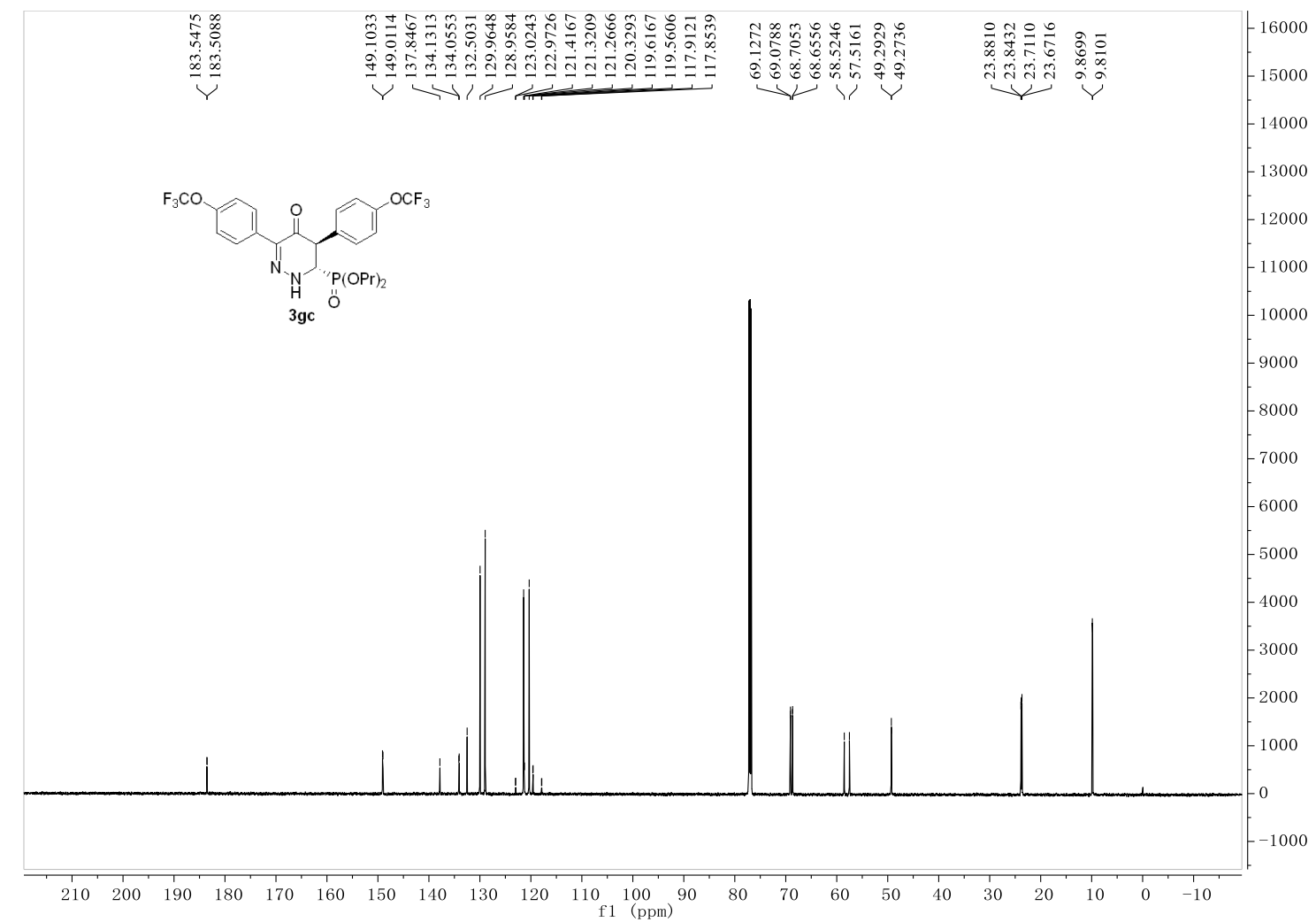


$600 \mathrm{MHz}, \mathrm{CDCl}_{3},{ }^{1} \mathrm{H} \mathrm{NMR}$

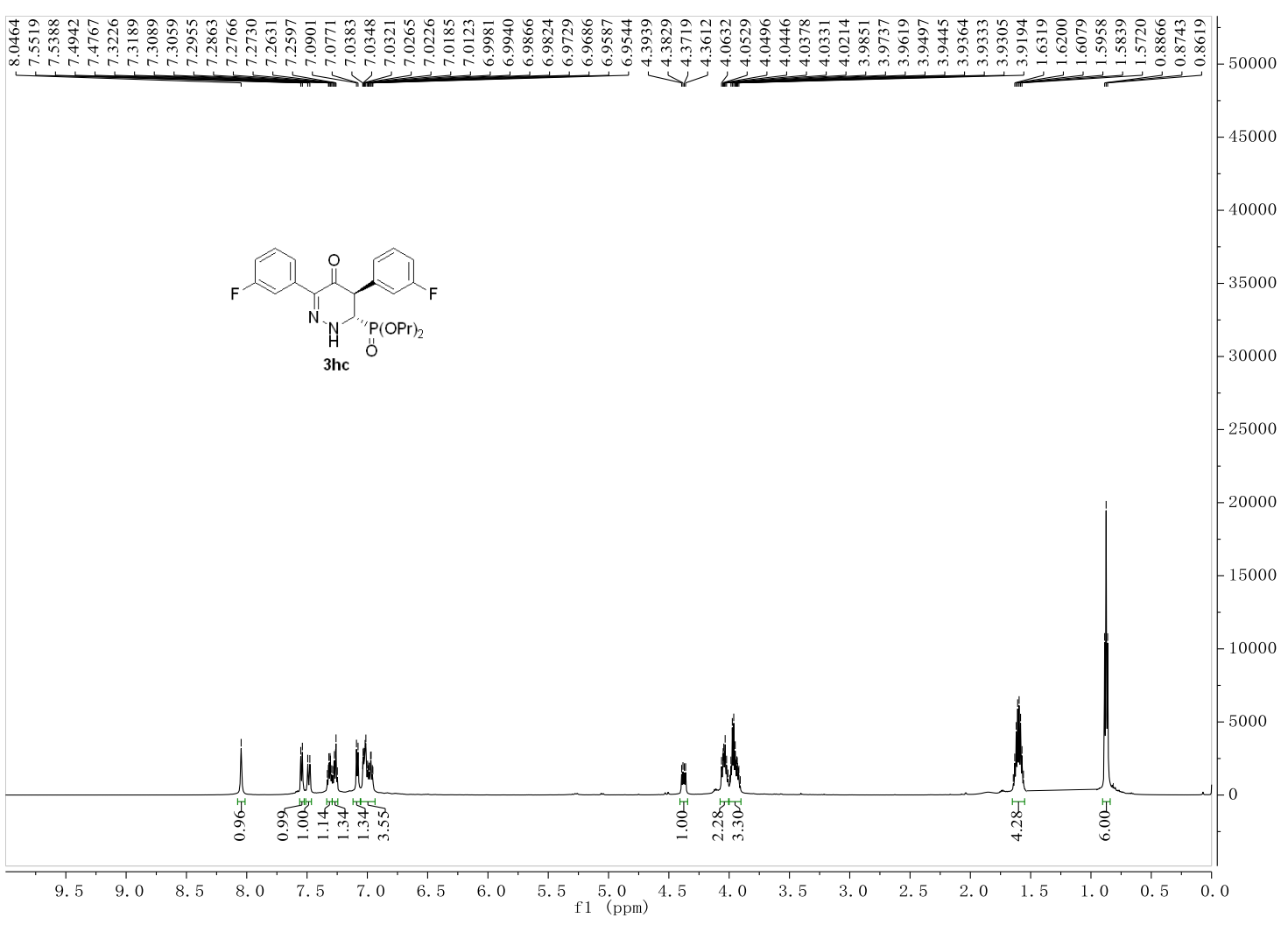

$151 \mathrm{MHz}, \mathrm{CDCl}_{3},{ }^{13} \mathrm{C} \mathrm{NMR}$

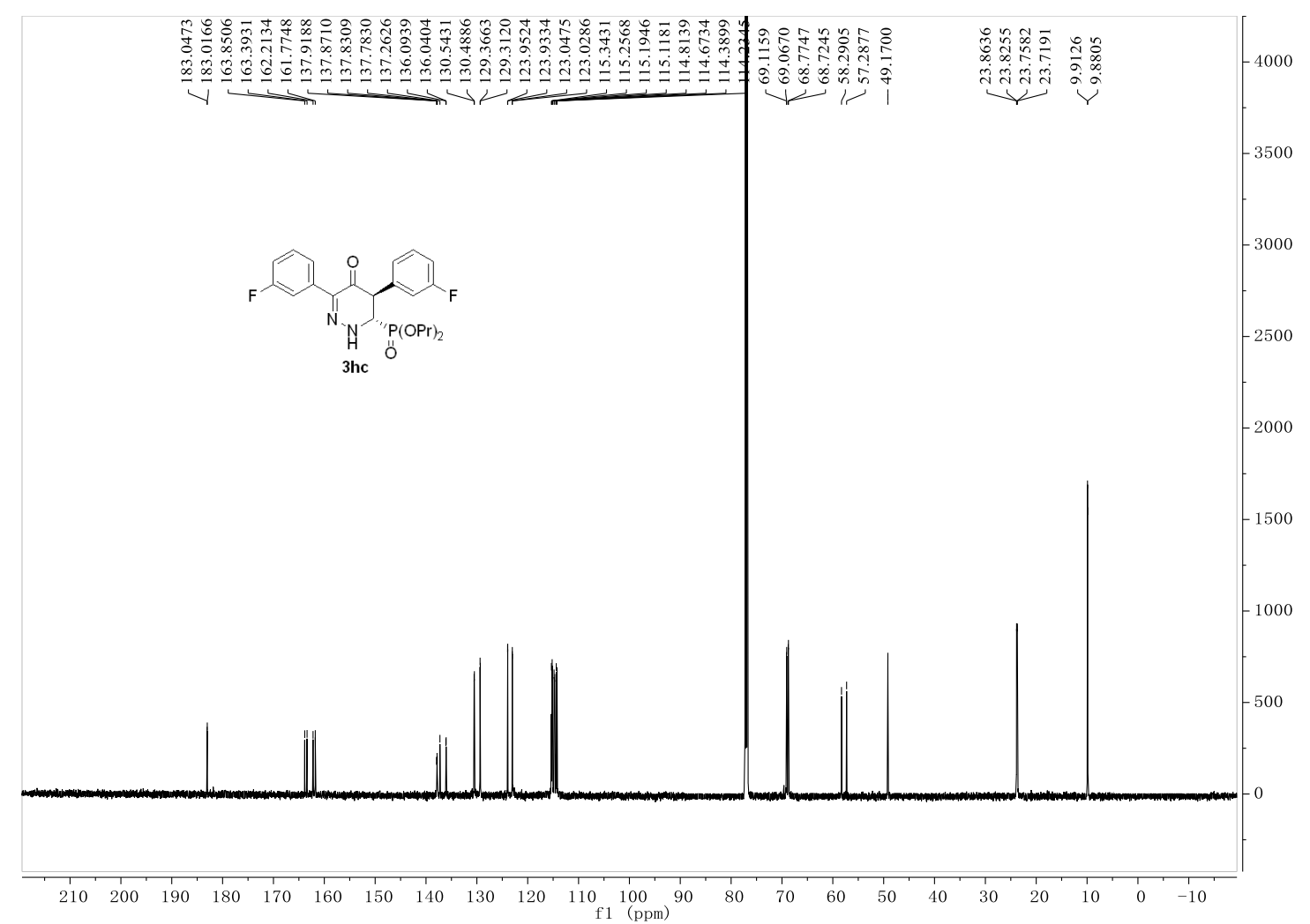




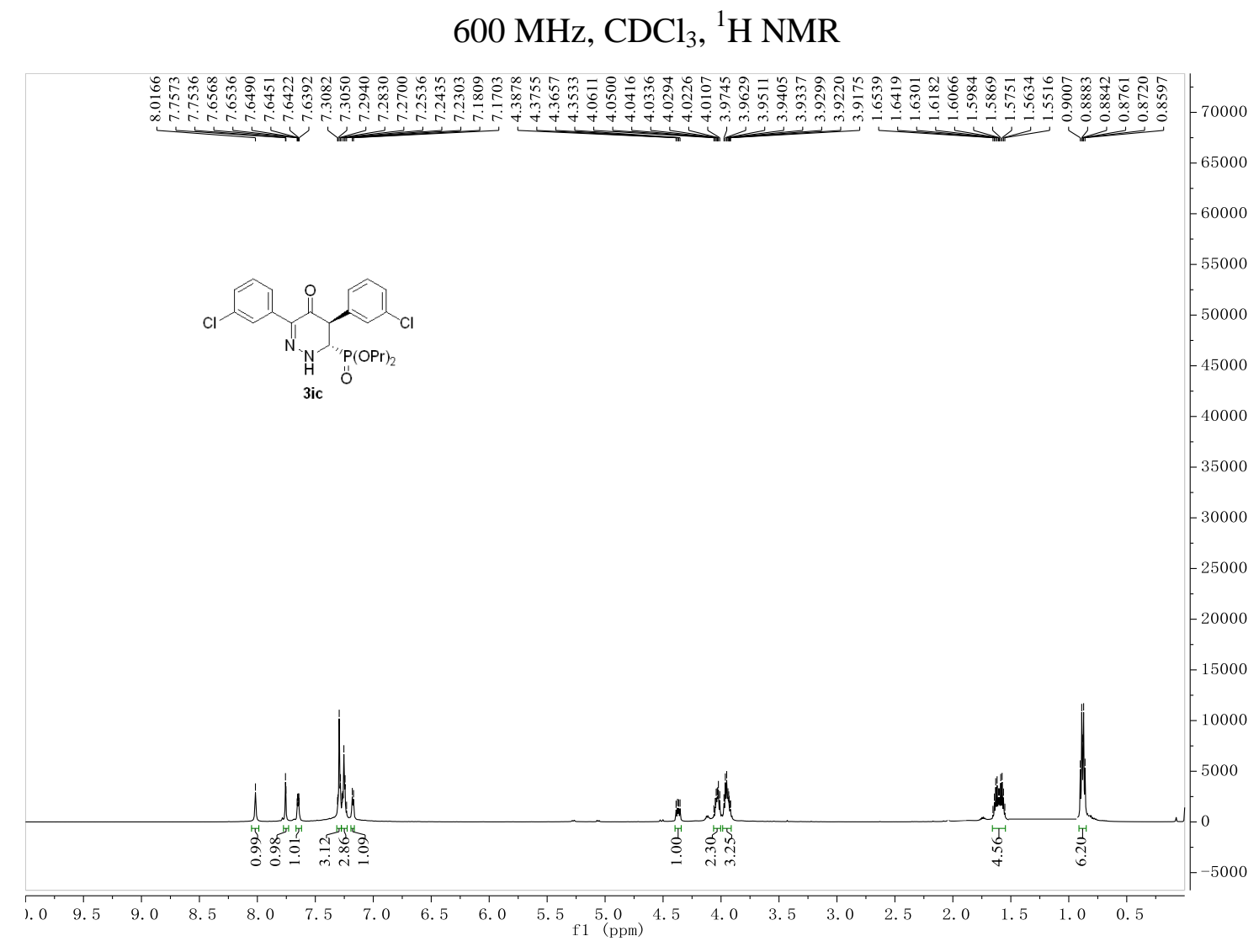

$151 \mathrm{MHz}, \mathrm{CDCl}_{3},{ }^{13} \mathrm{C} \mathrm{NMR}$

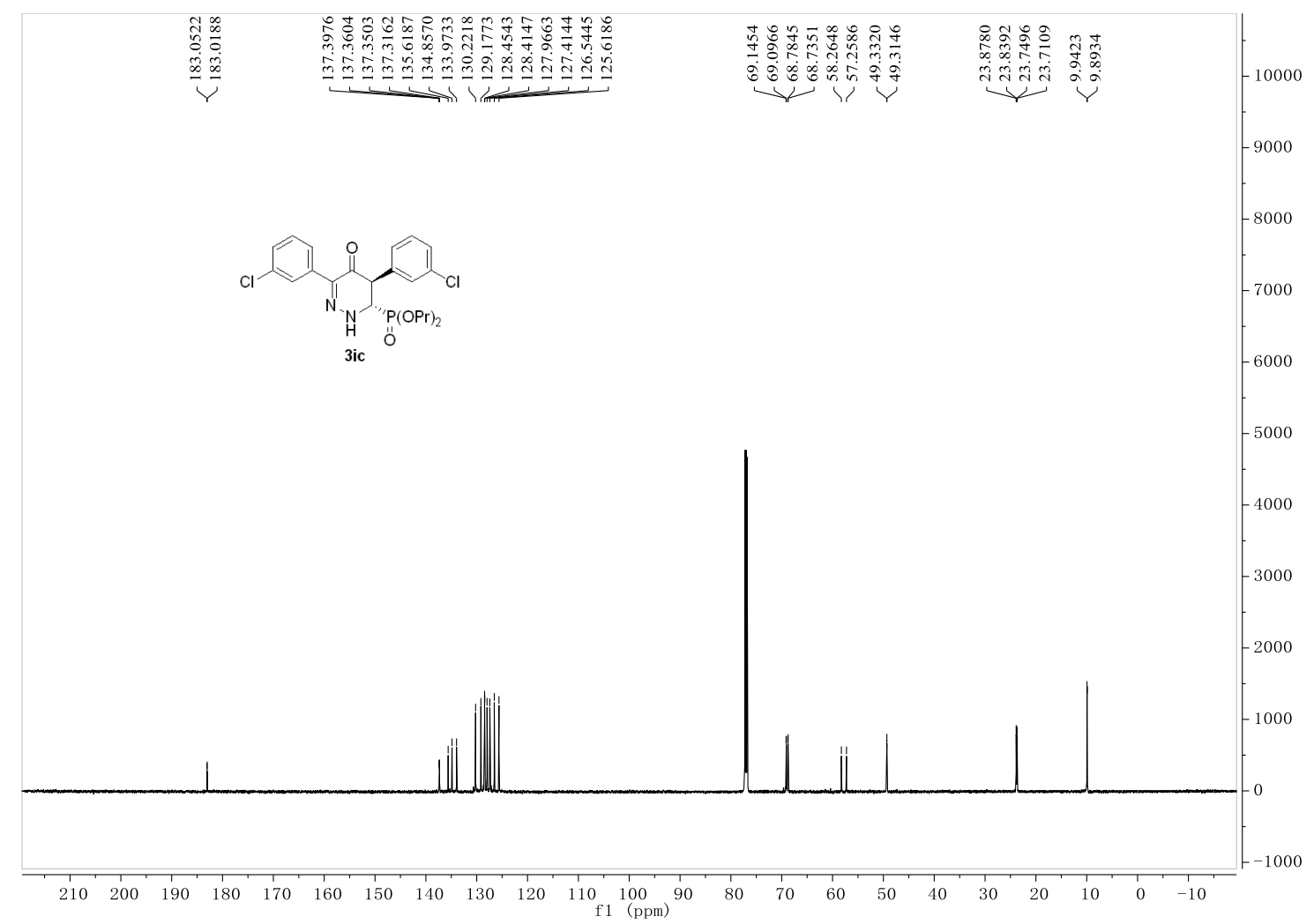




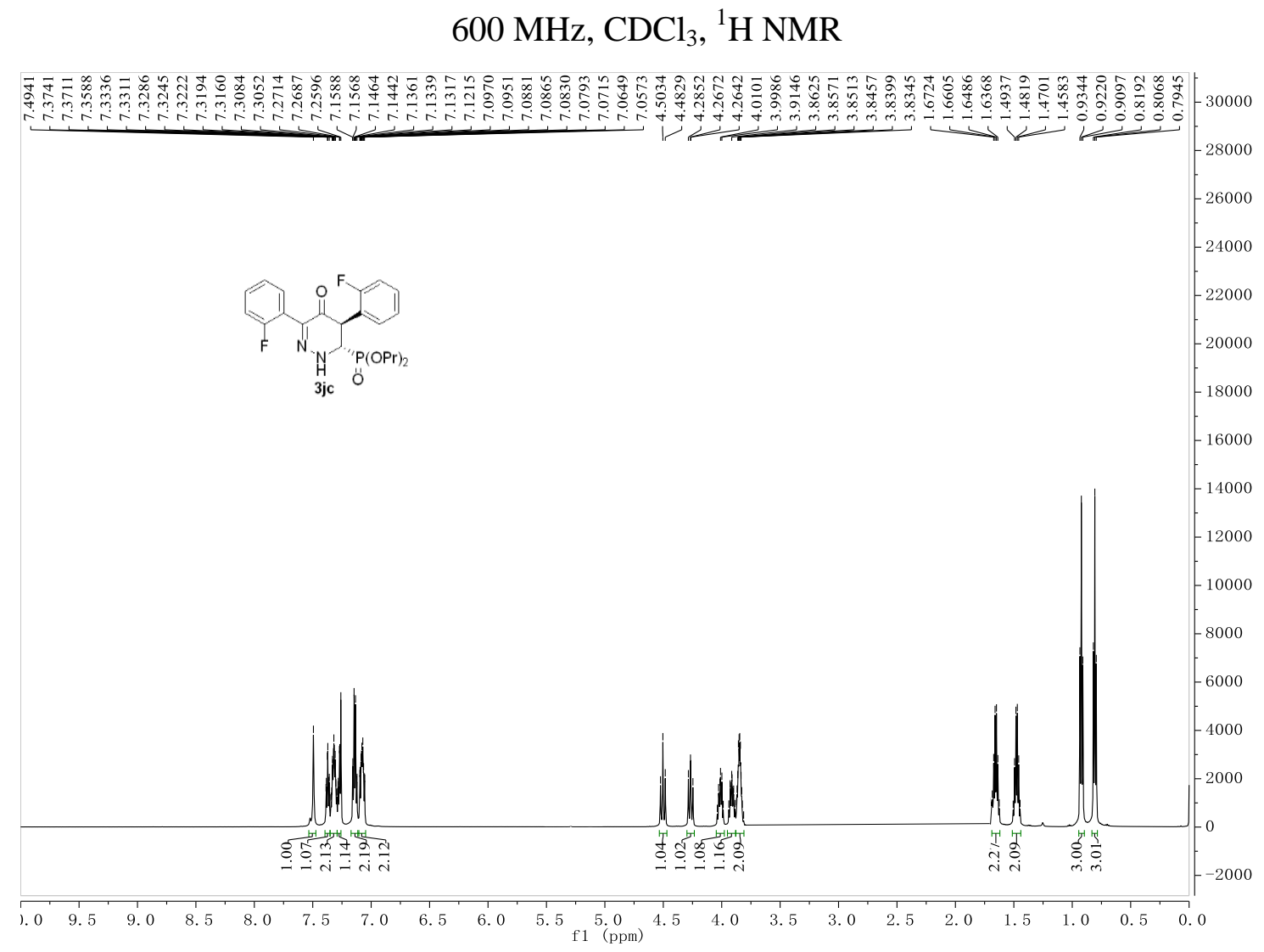

$151 \mathrm{MHz}, \mathrm{CDCl}_{3},{ }^{13} \mathrm{C}$ NMR

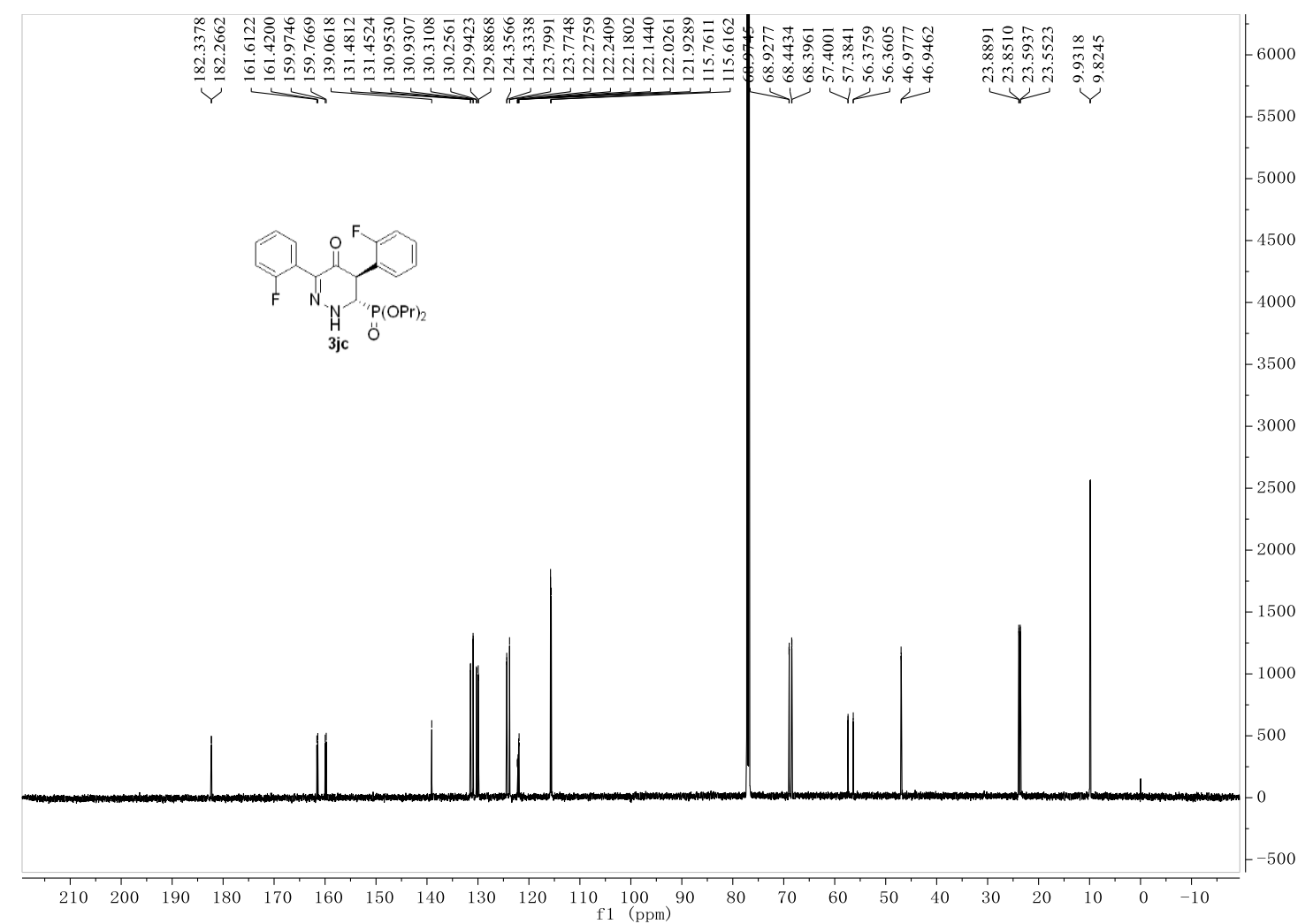


$600 \mathrm{MHz}, \mathrm{CDCl}_{3},{ }^{1} \mathrm{H} \mathrm{NMR}$

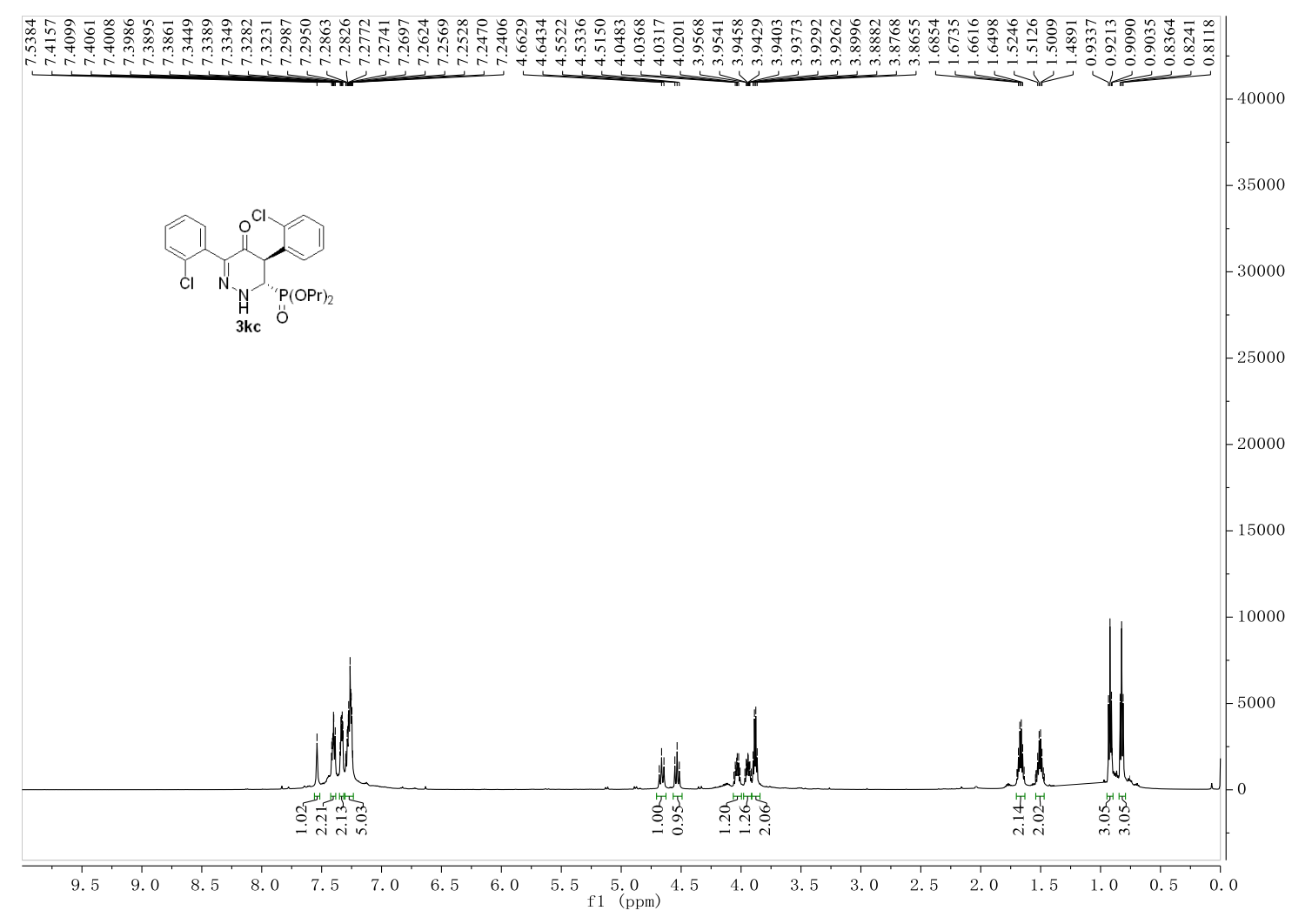

$151 \mathrm{MHz}, \mathrm{CDCl}_{3},{ }^{13} \mathrm{C} \mathrm{NMR}$

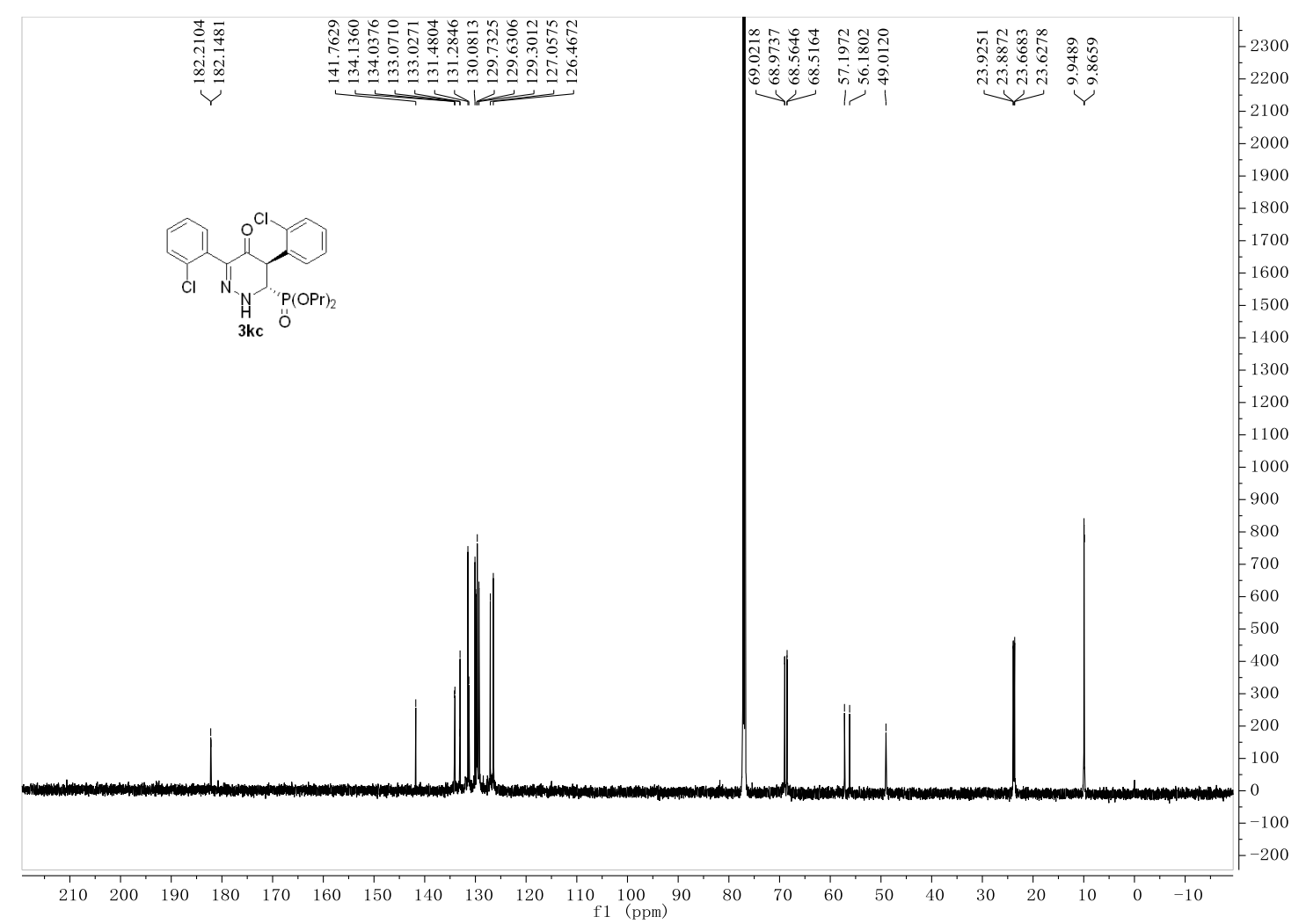




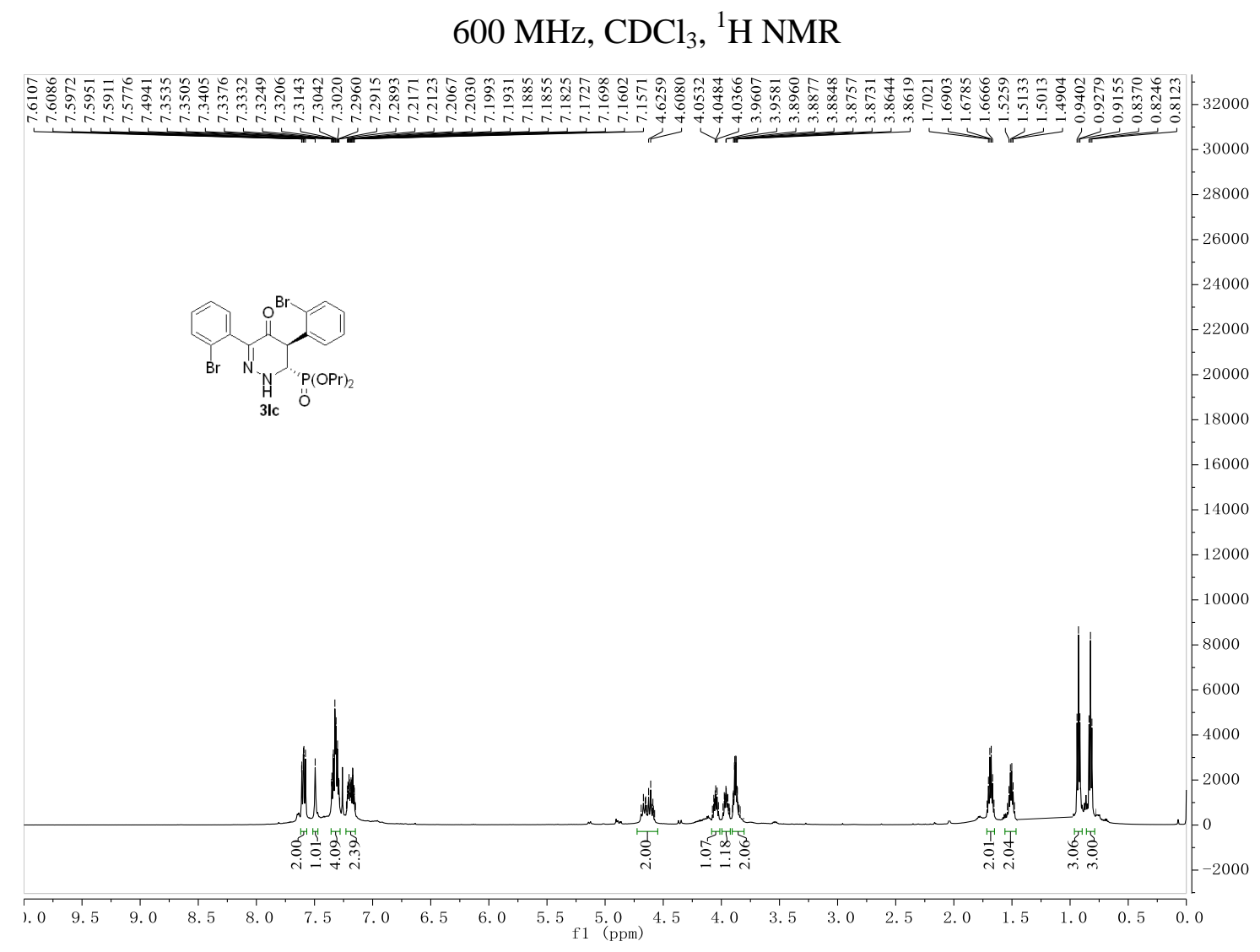

$151 \mathrm{MHz}, \mathrm{CDCl}_{3},{ }^{13} \mathrm{C} \mathrm{NMR}$

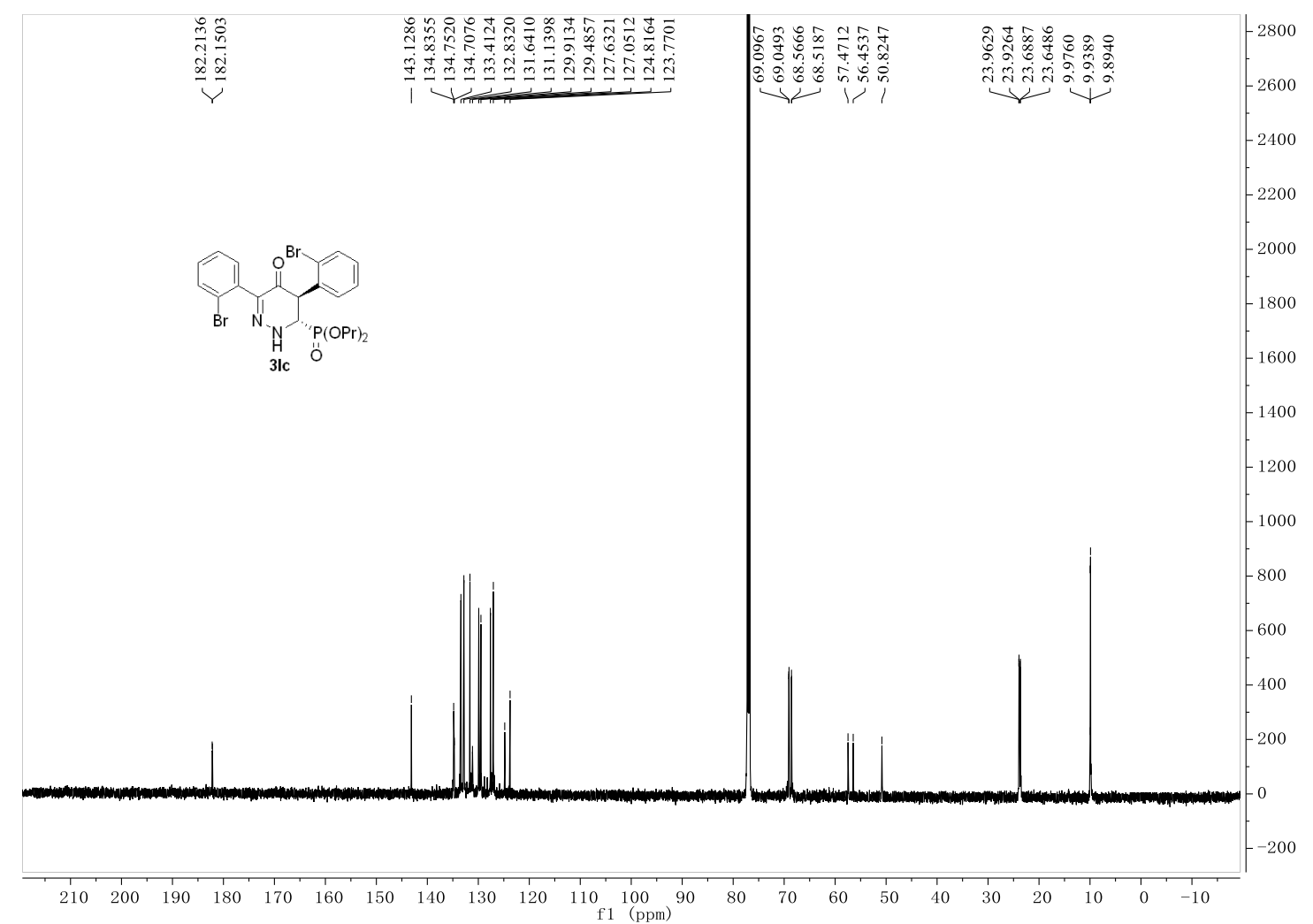




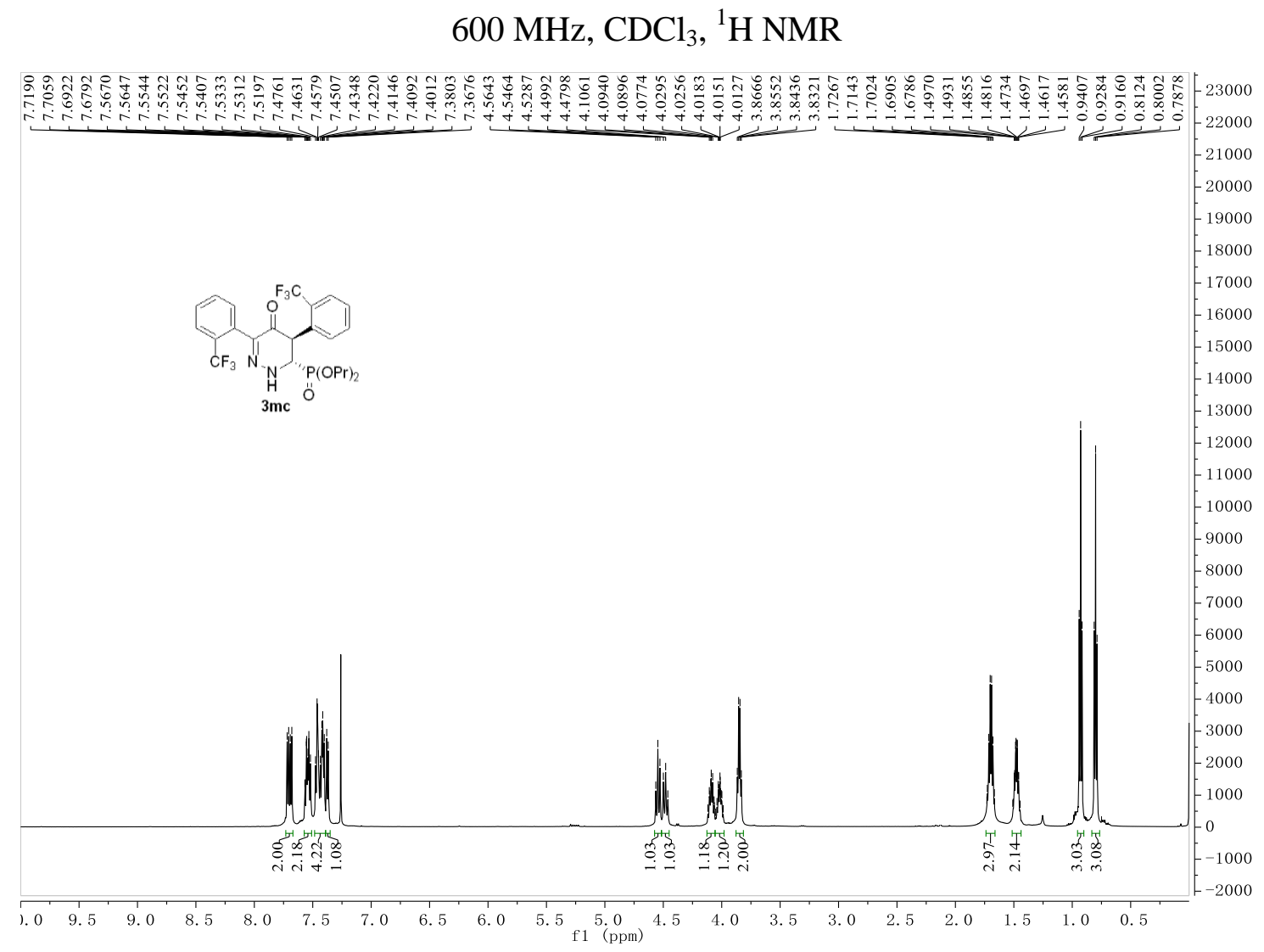

$151 \mathrm{MHz}, \mathrm{CDCl}_{3},{ }^{13} \mathrm{C} \mathrm{NMR}$

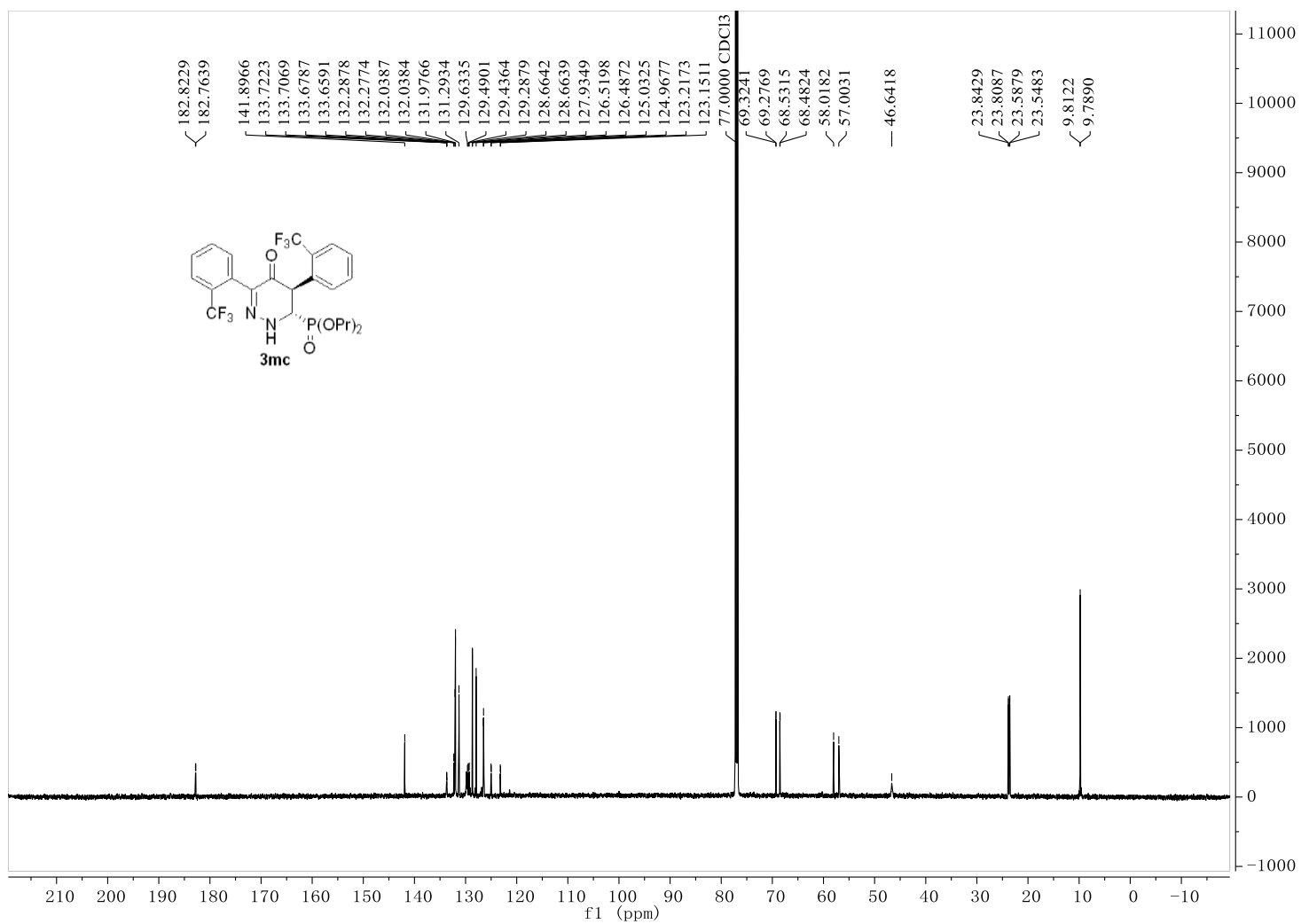




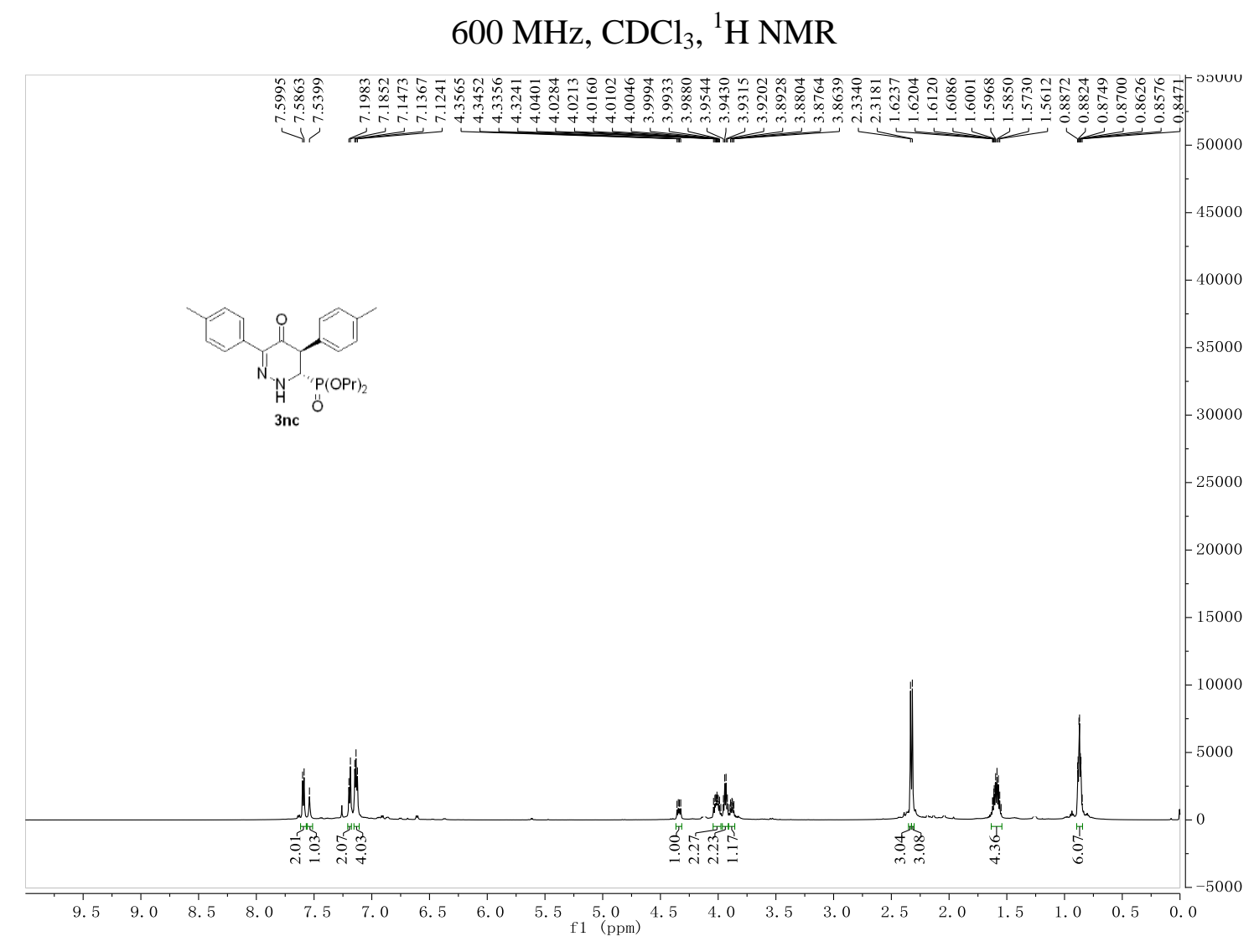

$151 \mathrm{MHz}, \mathrm{CDCl}_{3},{ }^{13} \mathrm{C} \mathrm{NMR}$

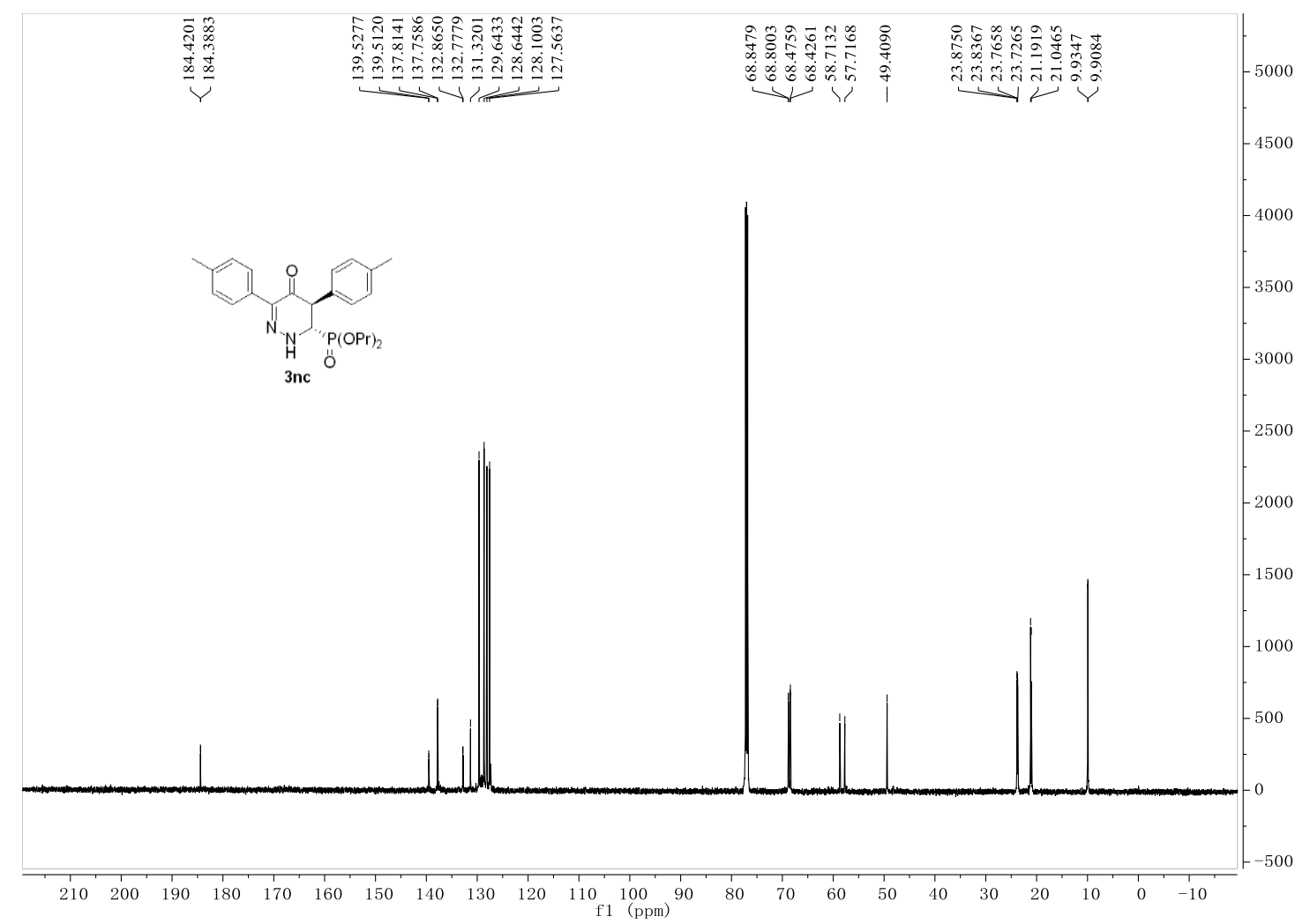


$600 \mathrm{MHz}, \mathrm{CDCl}_{3},{ }^{1} \mathrm{H} \mathrm{NMR}$

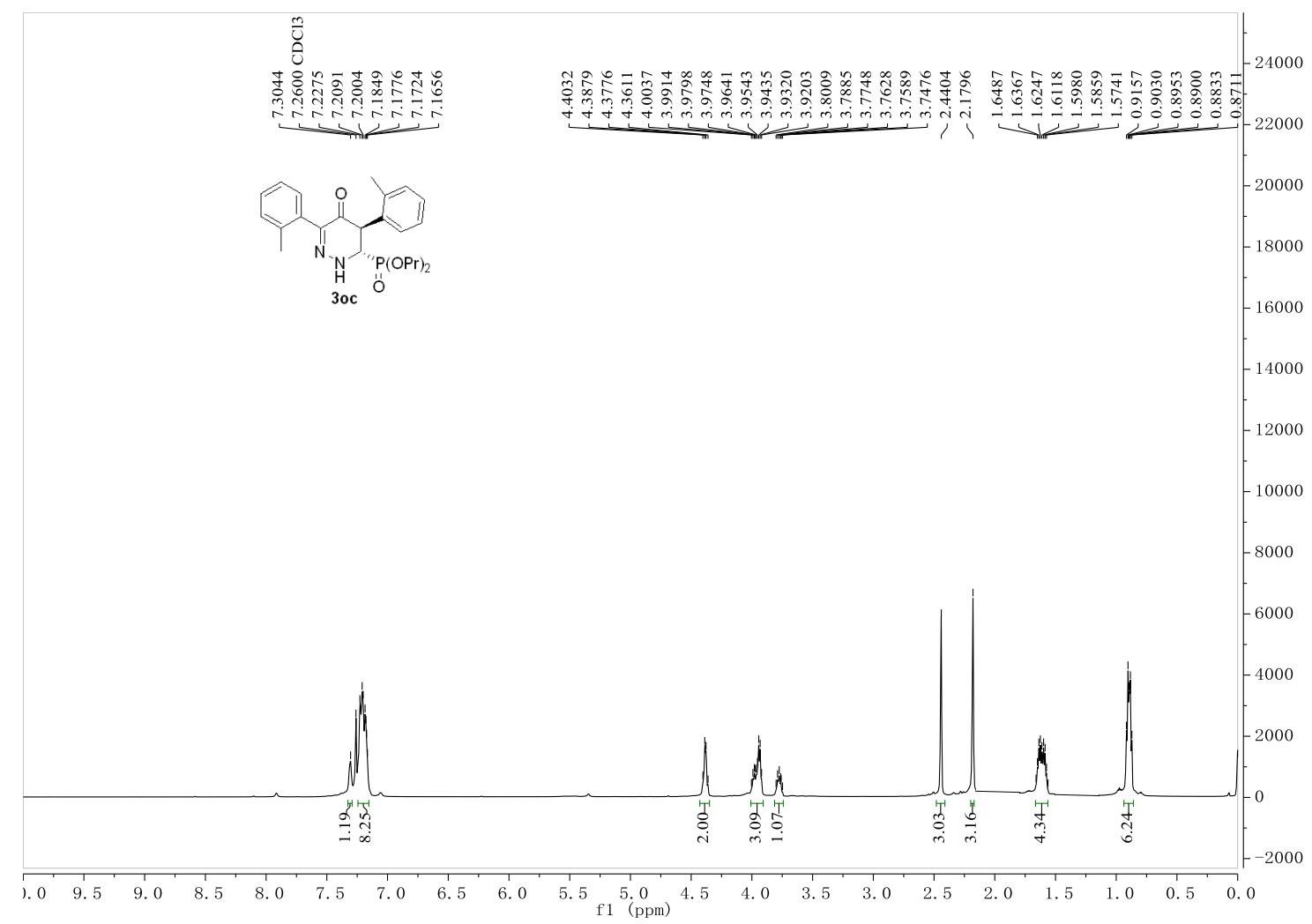

$151 \mathrm{MHz}, \mathrm{CDCl}_{3},{ }^{13} \mathrm{C} \mathrm{NMR}$

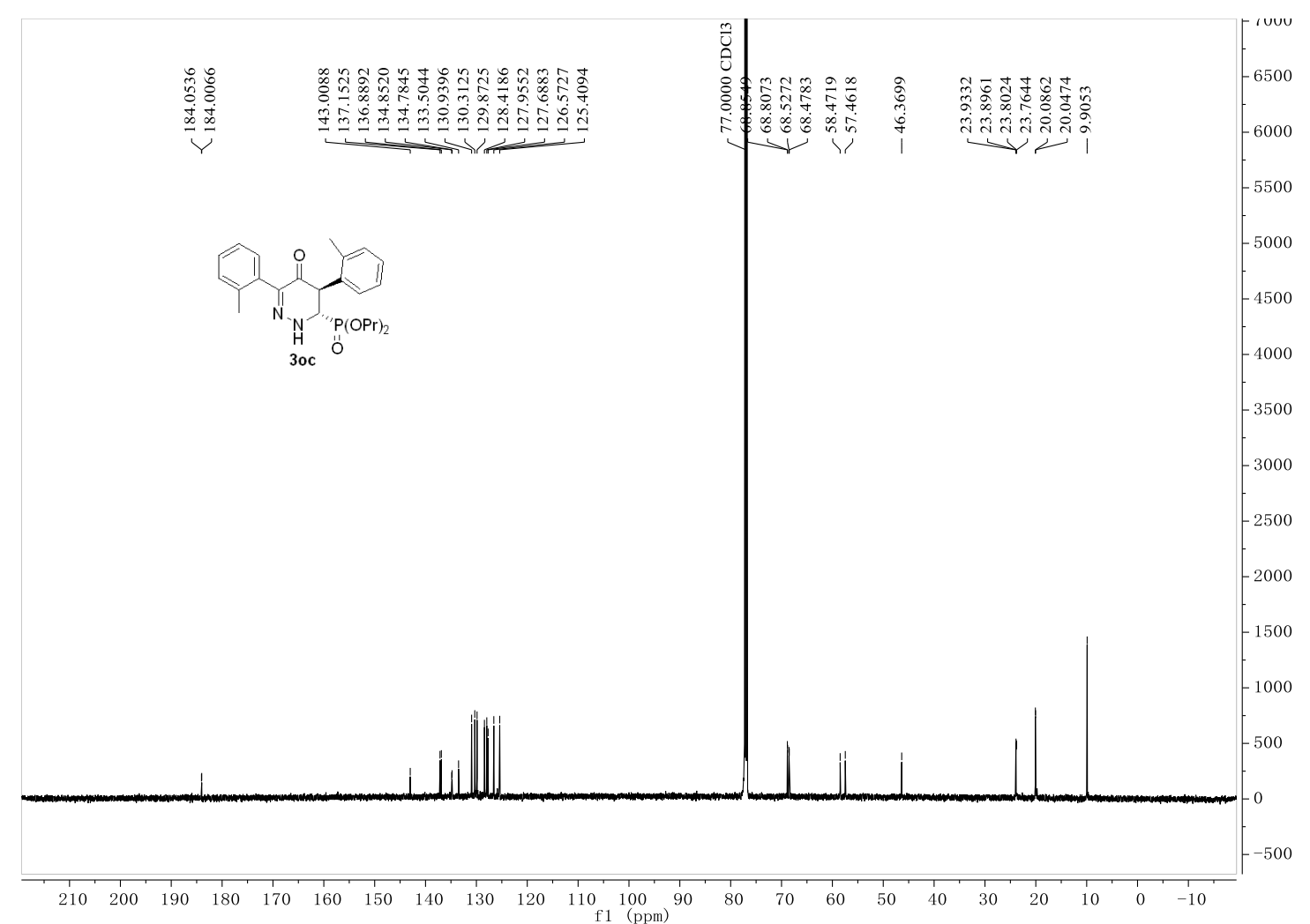


$600 \mathrm{MHz}, \mathrm{CDCl}_{3},{ }^{1} \mathrm{H} \mathrm{NMR}$

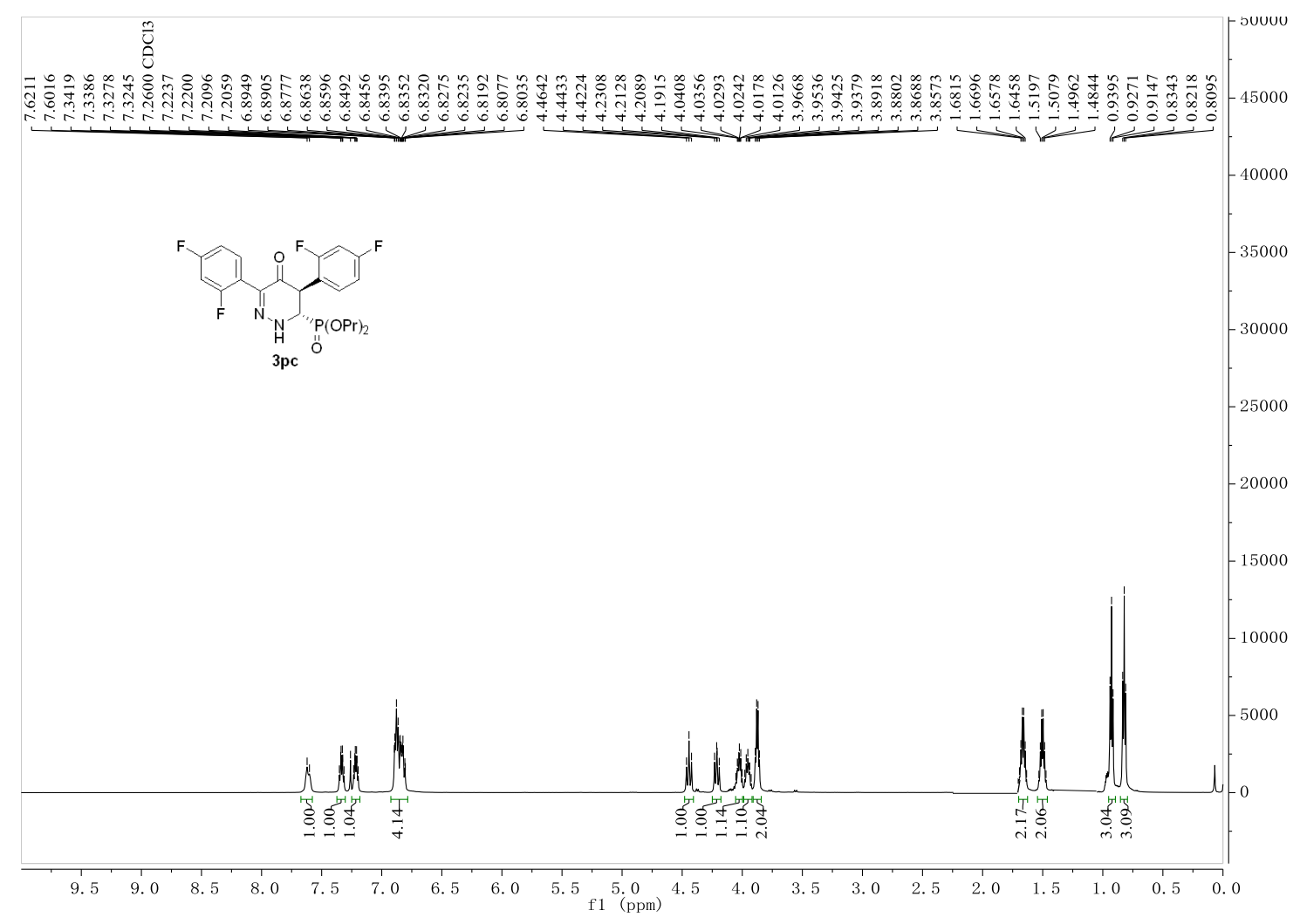

$151 \mathrm{MHz}, \mathrm{CDCl}_{3},{ }^{13} \mathrm{C} \mathrm{NMR}$

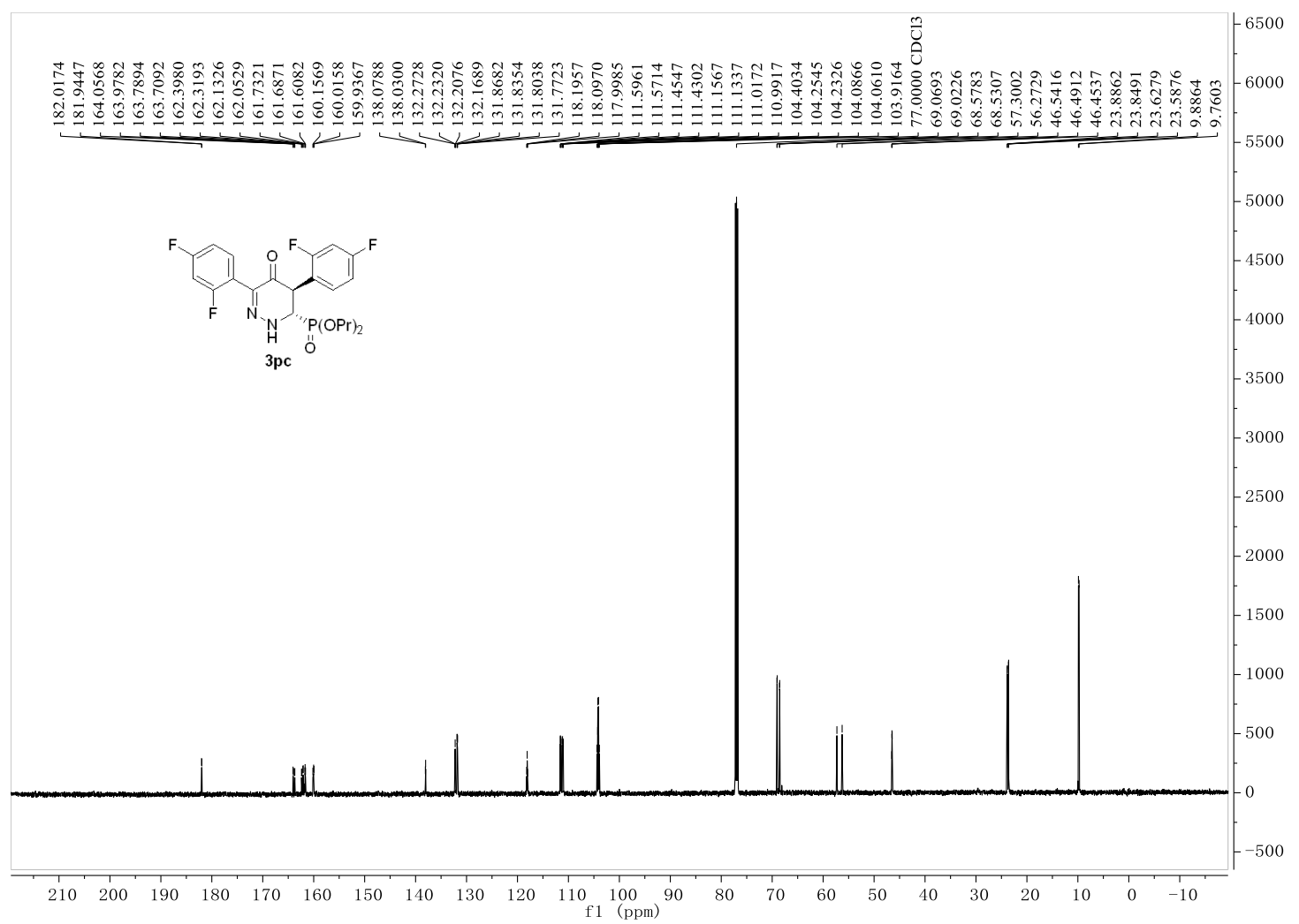


$600 \mathrm{MHz}, \mathrm{DMSO}-d_{6},{ }^{1} \mathrm{H}$ NMR

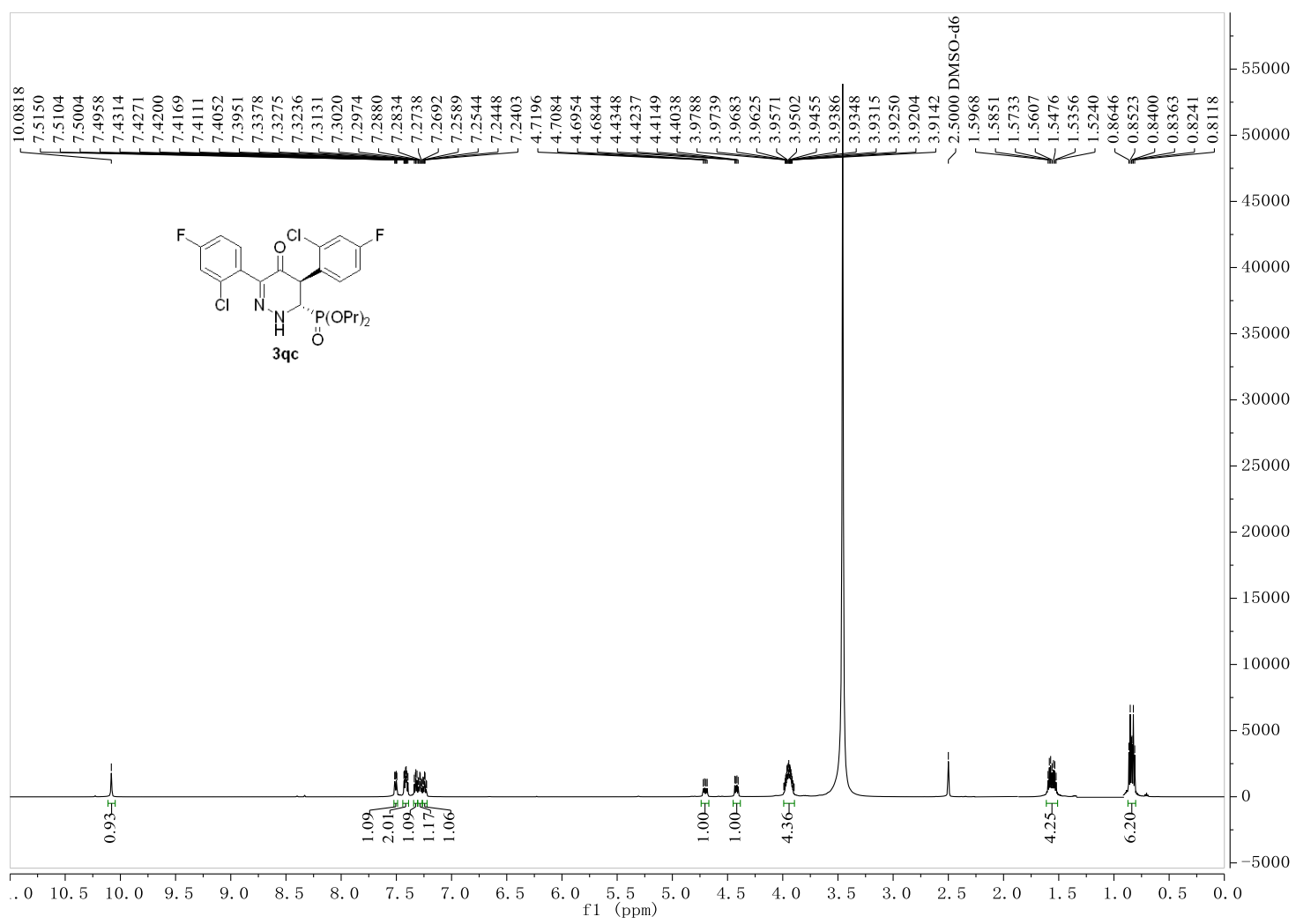

$151 \mathrm{MHz}$, DMSO- $d_{3},{ }^{13} \mathrm{C}$ NMR

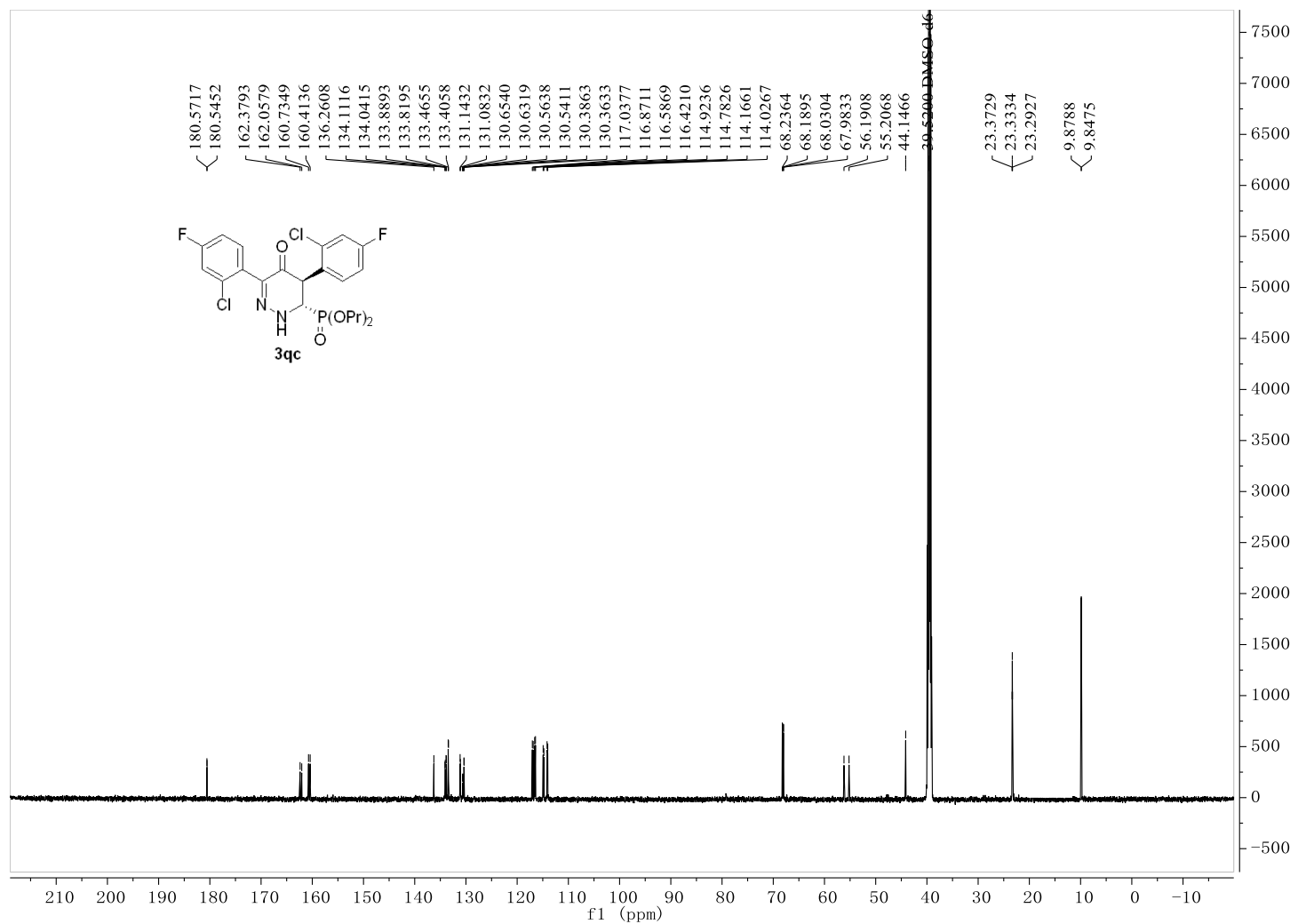


$600 \mathrm{MHz}, \mathrm{CDCl}_{3},{ }^{1} \mathrm{H}$ NMR

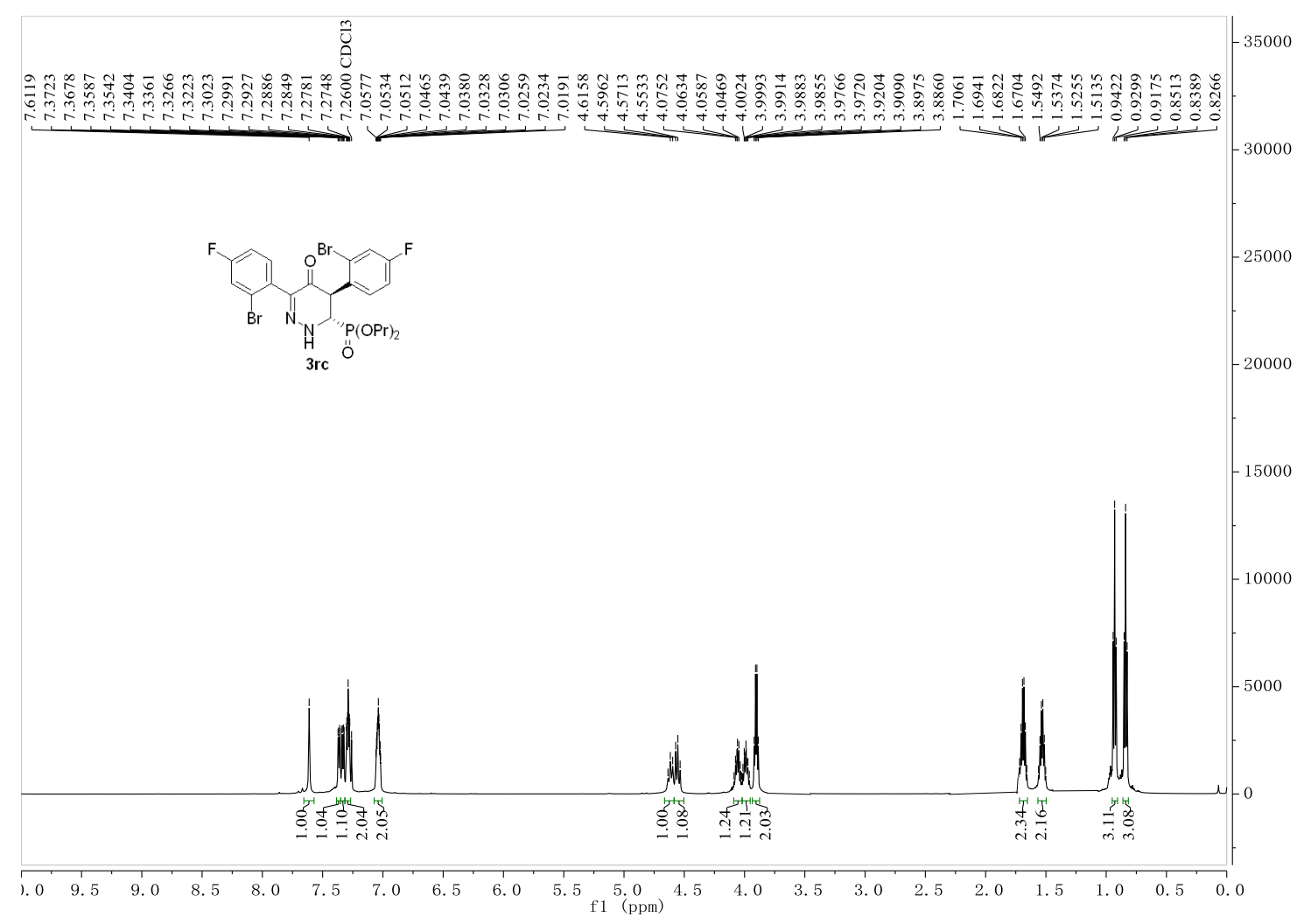

$151 \mathrm{MHz}, \mathrm{CDCl}_{3},{ }^{13} \mathrm{C} \mathrm{NMR}$

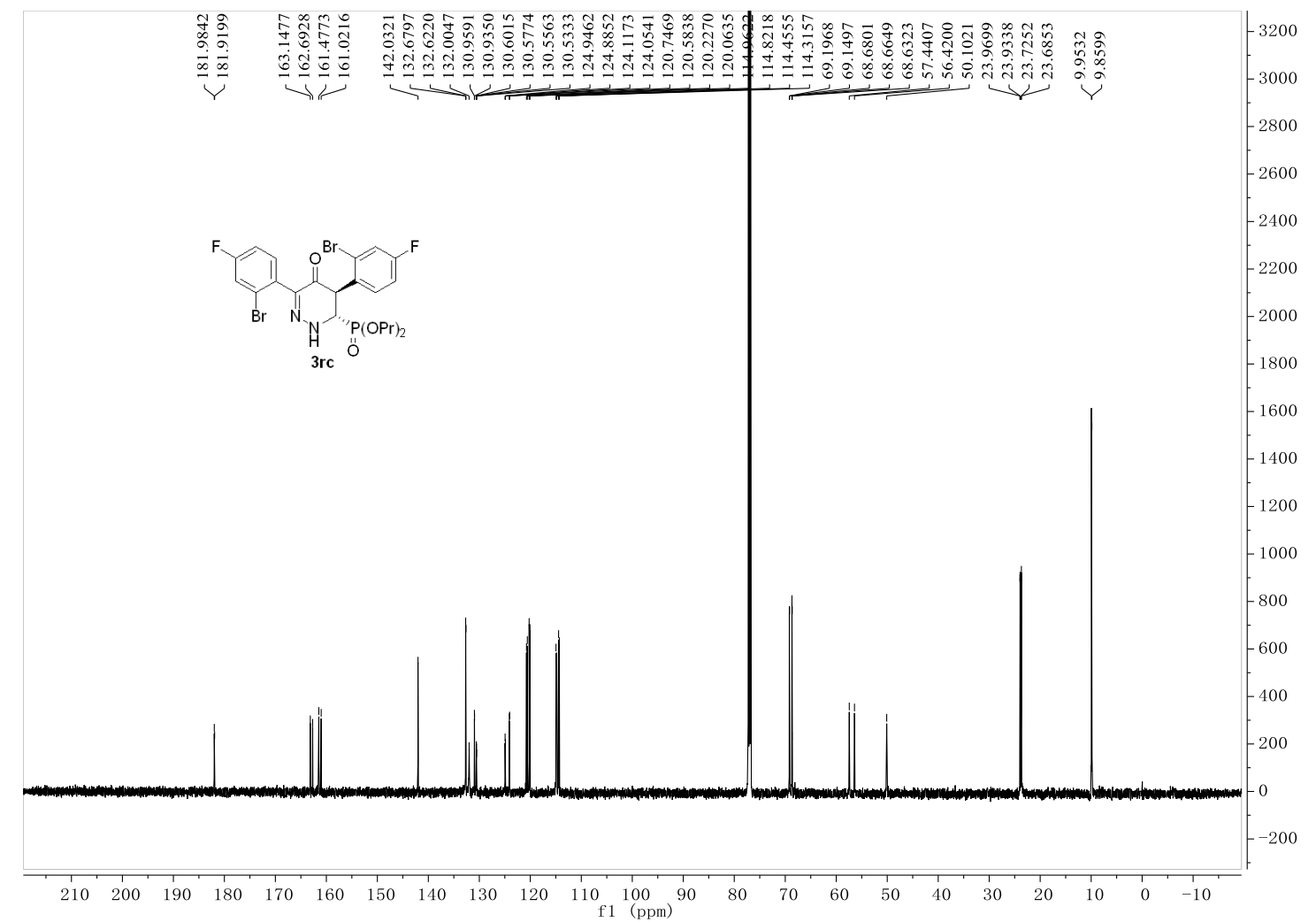


$600 \mathrm{MHz}, \mathrm{CDCl}_{3},{ }^{1} \mathrm{H} \mathrm{NMR}$

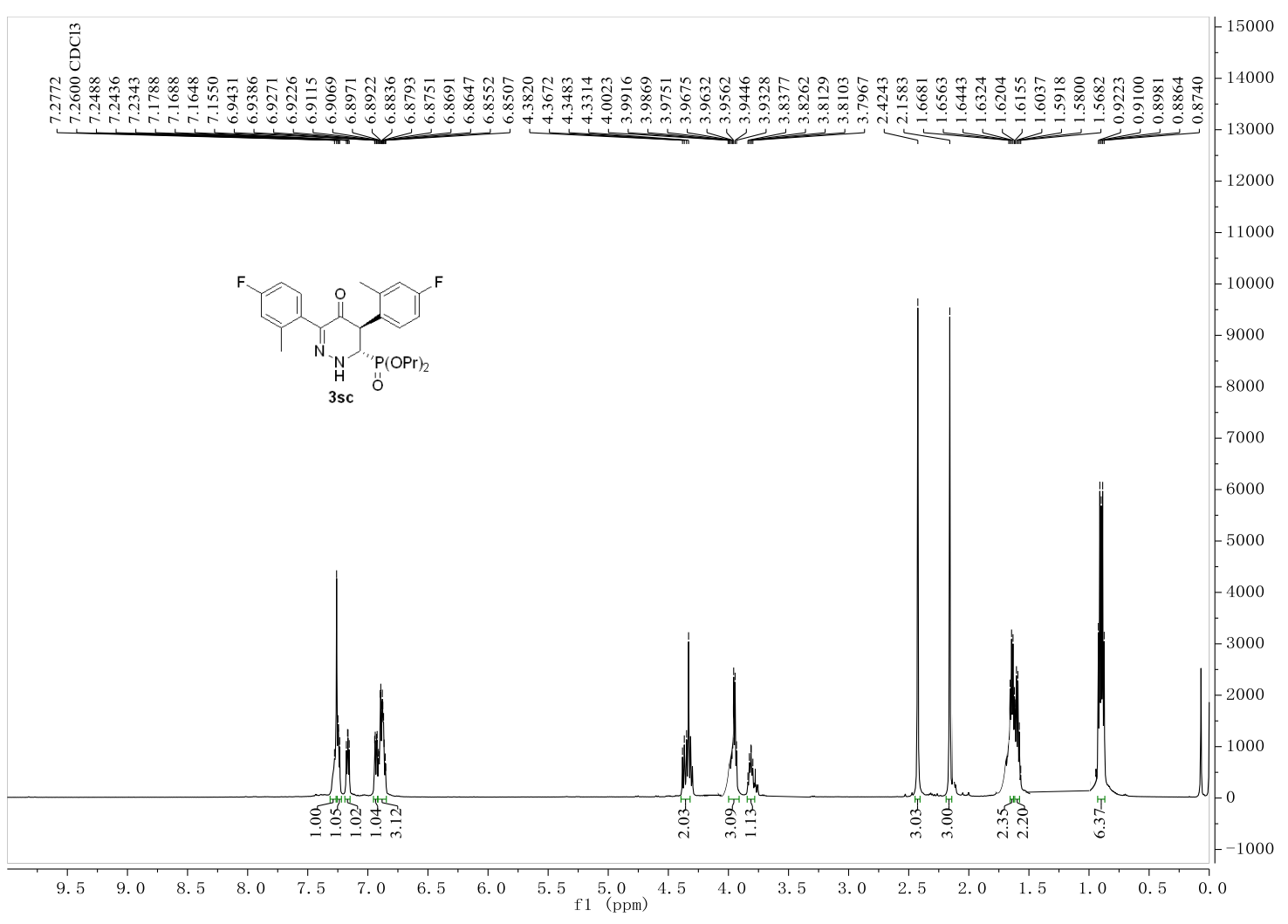

$151 \mathrm{MHz}, \mathrm{CDCl}_{3},{ }^{13} \mathrm{C}$ NMR

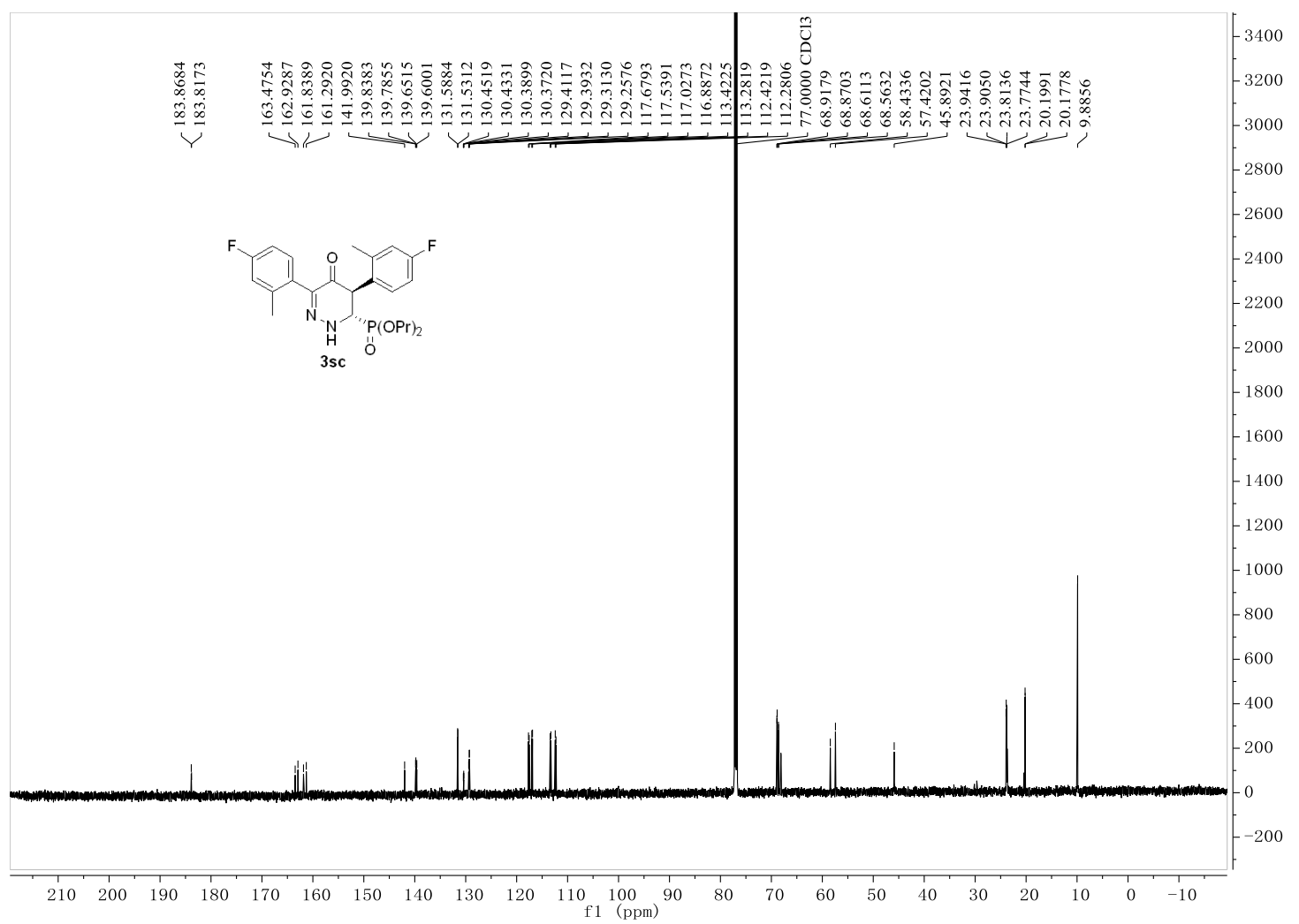




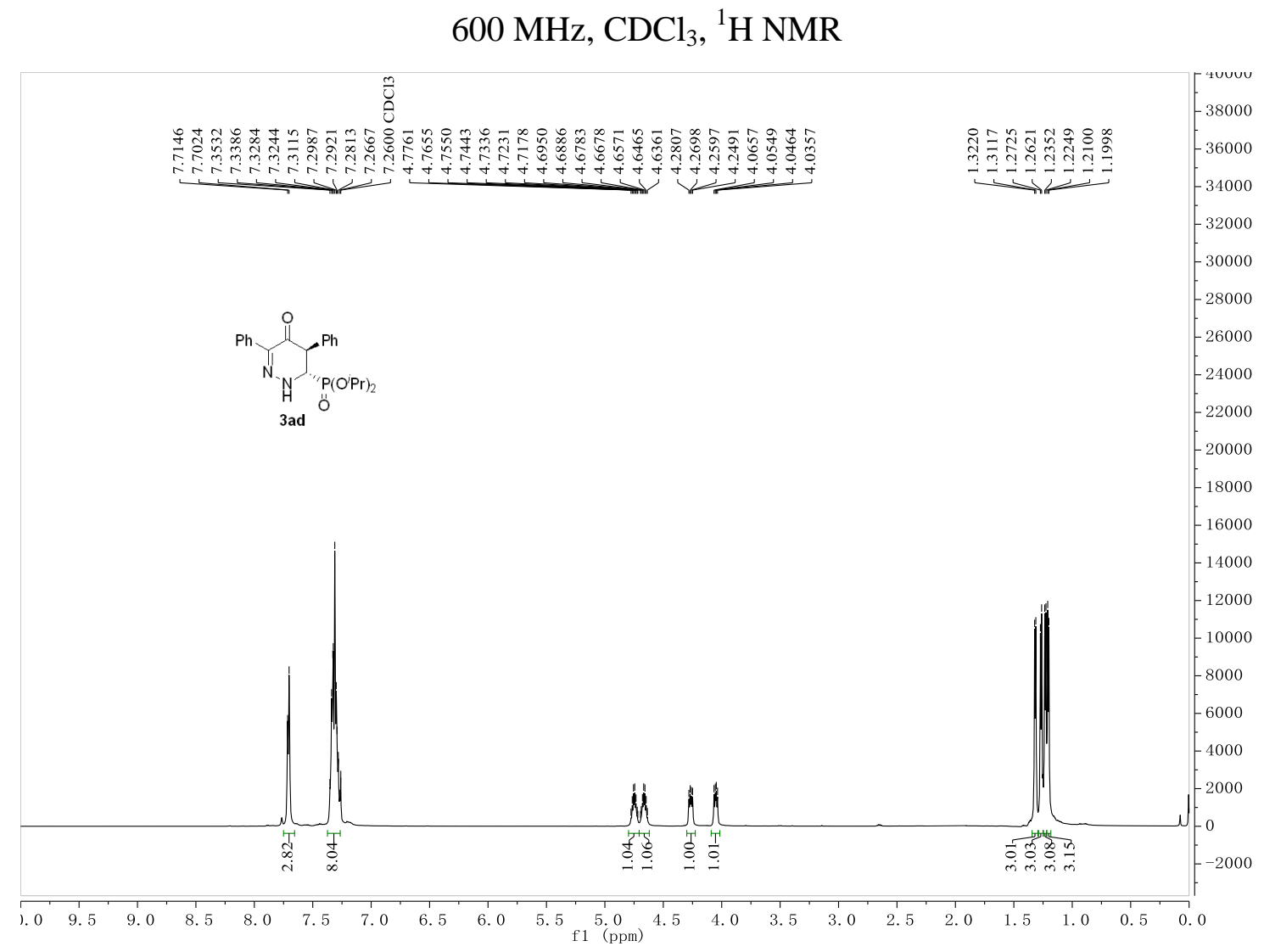

$151 \mathrm{MHz}, \mathrm{CDCl}_{3},{ }^{13} \mathrm{C} \mathrm{NMR}$

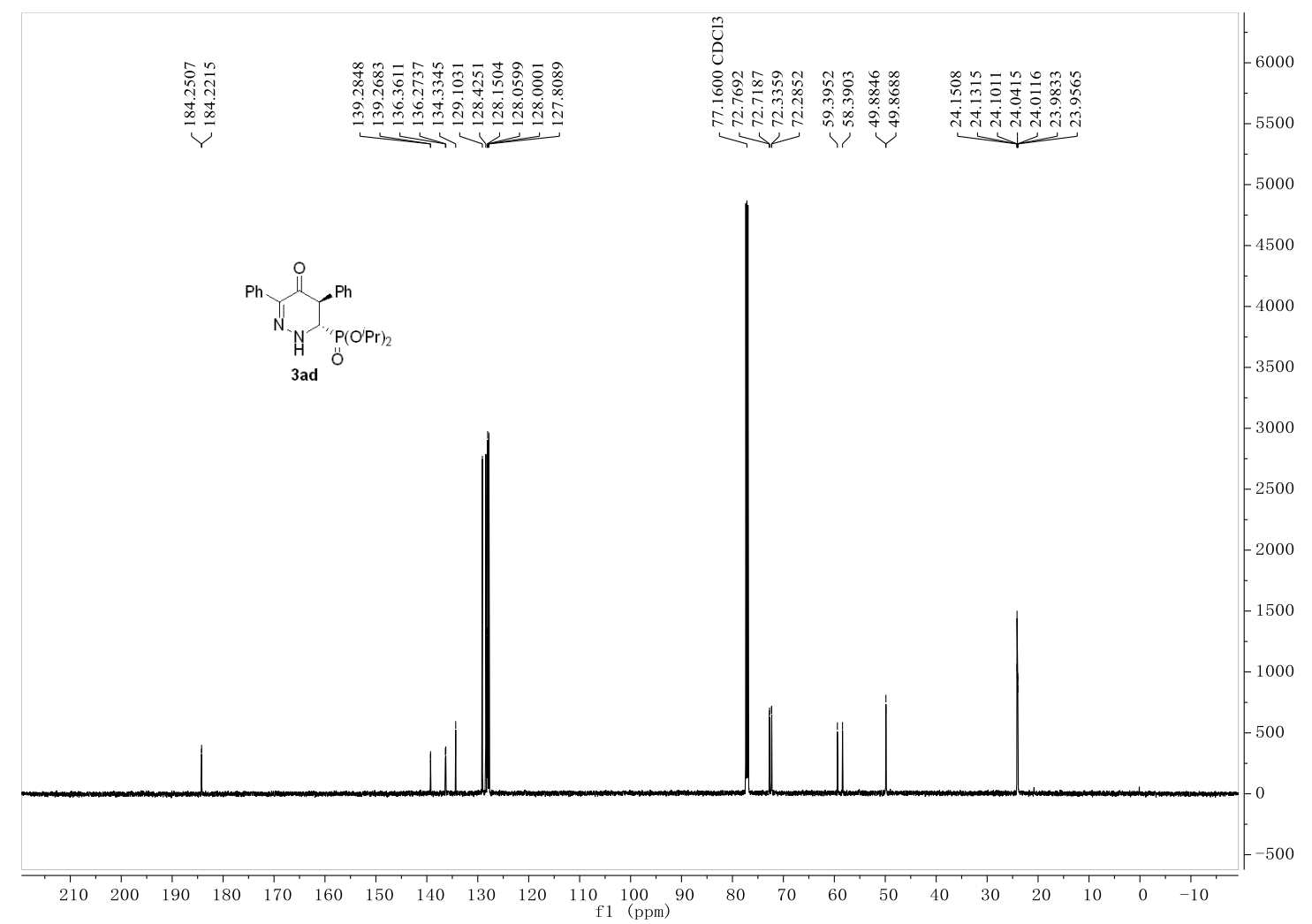




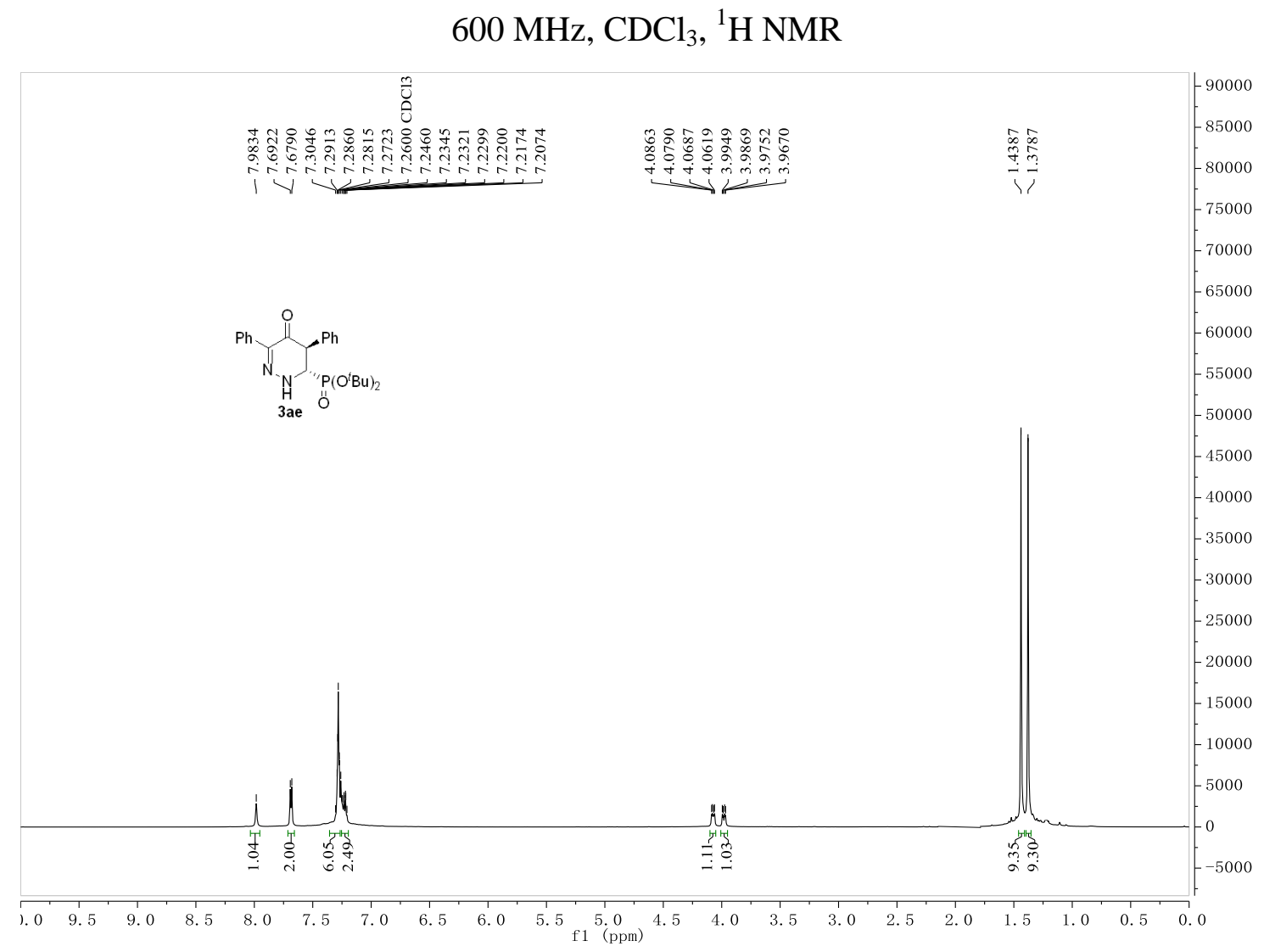

$151 \mathrm{MHz}, \mathrm{CDCl}_{3},{ }^{13} \mathrm{C} \mathrm{NMR}$

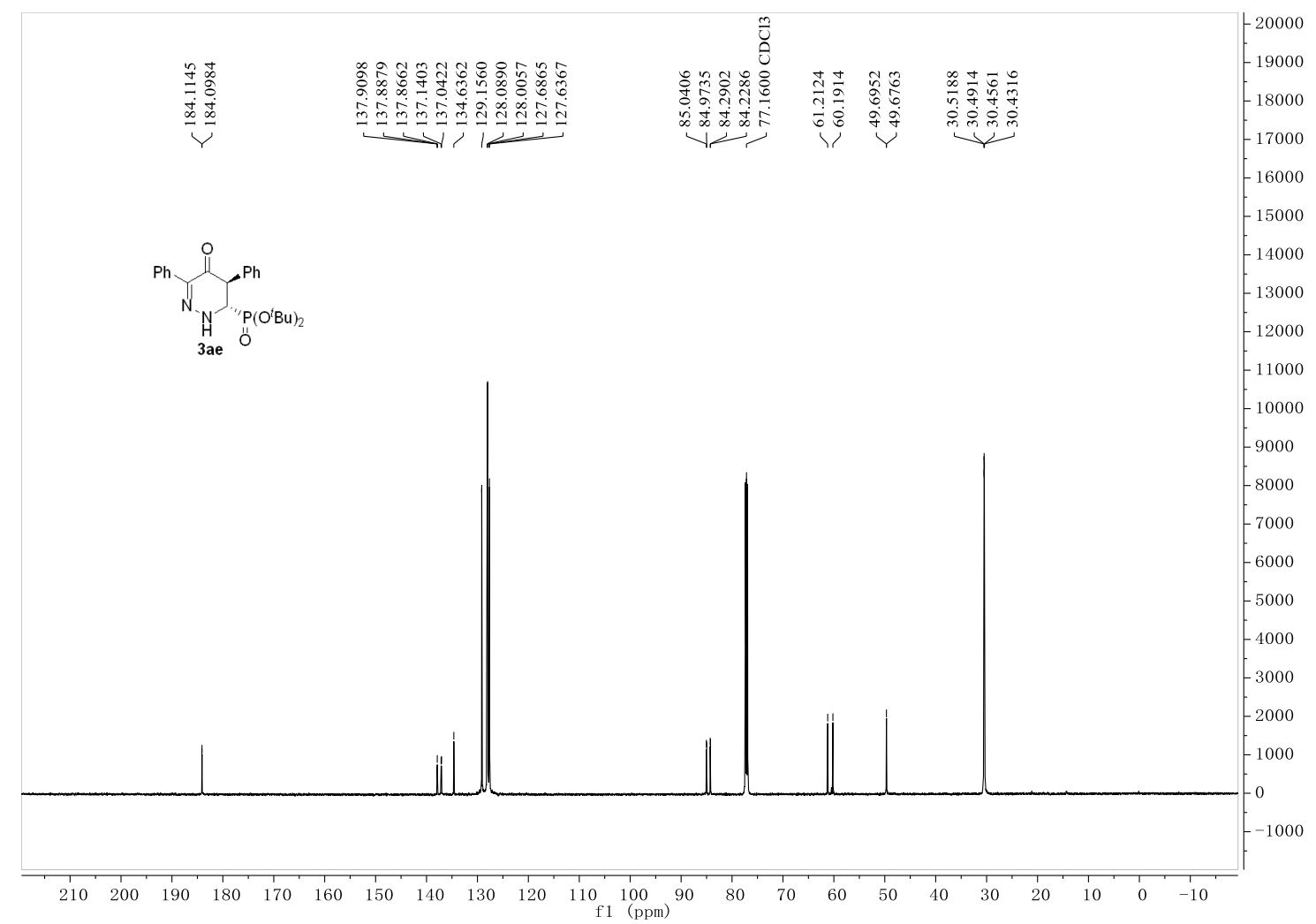




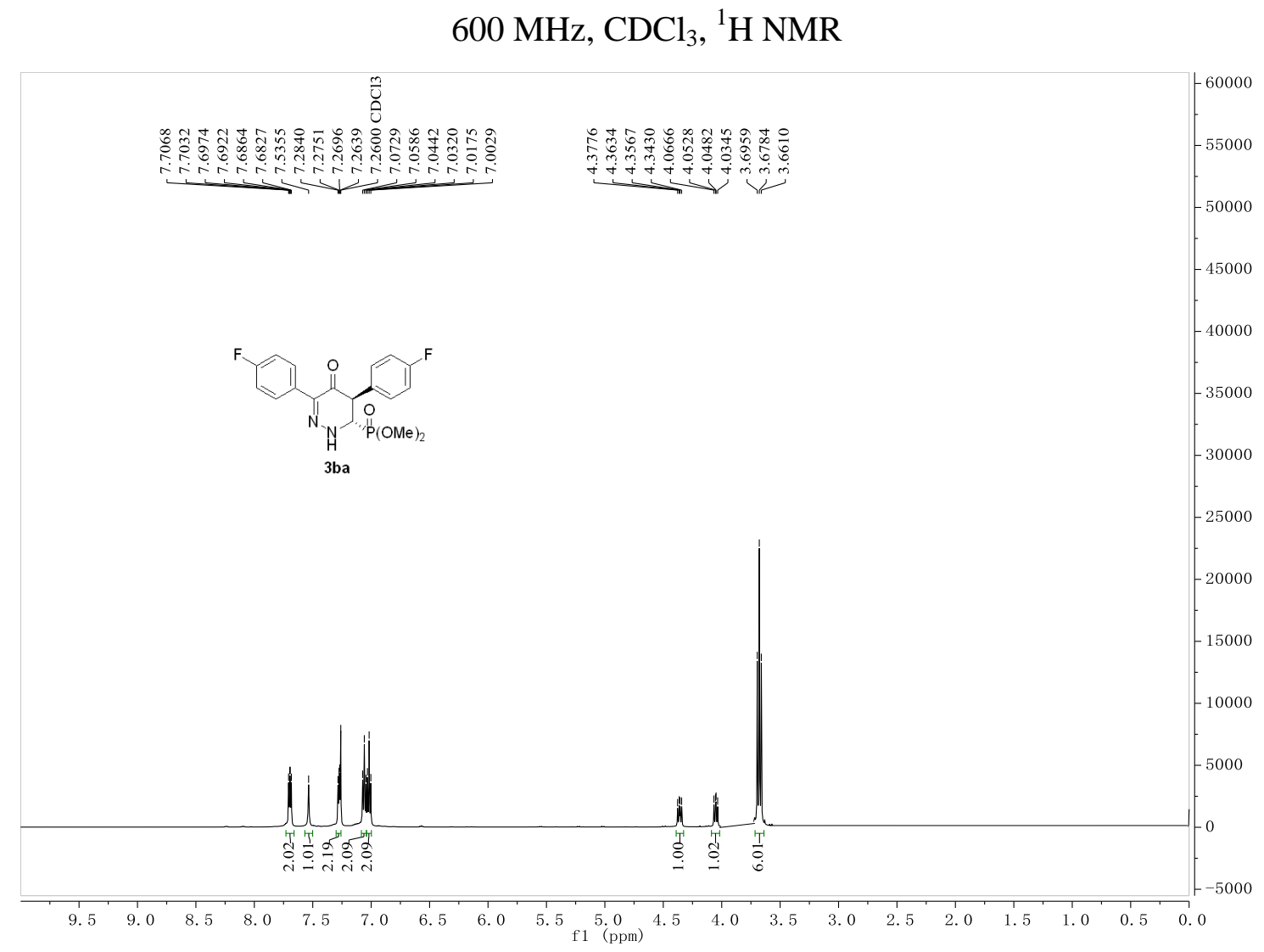

$151 \mathrm{MHz}, \mathrm{CDCl}_{3},{ }^{13} \mathrm{C} \mathrm{NMR}$

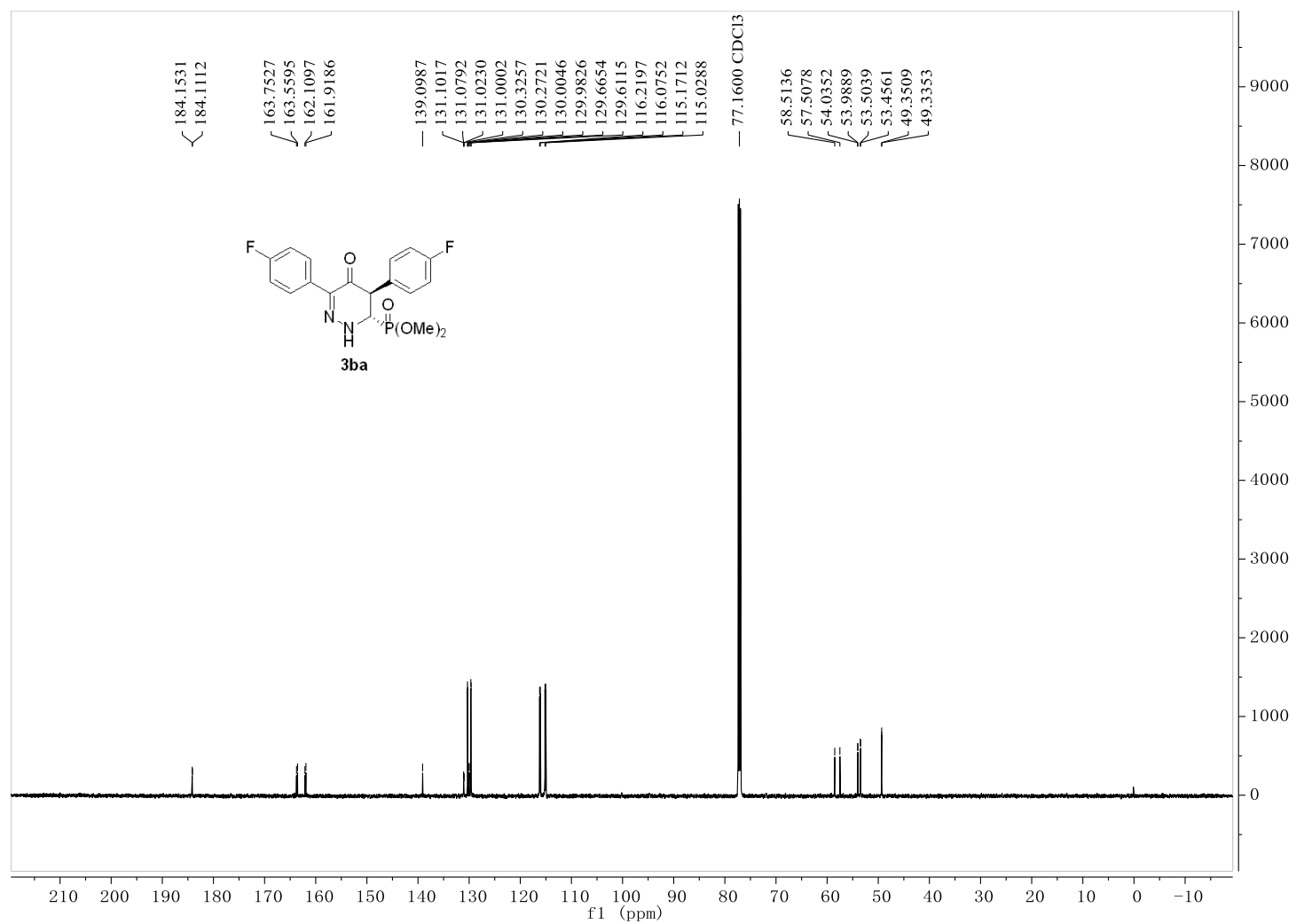




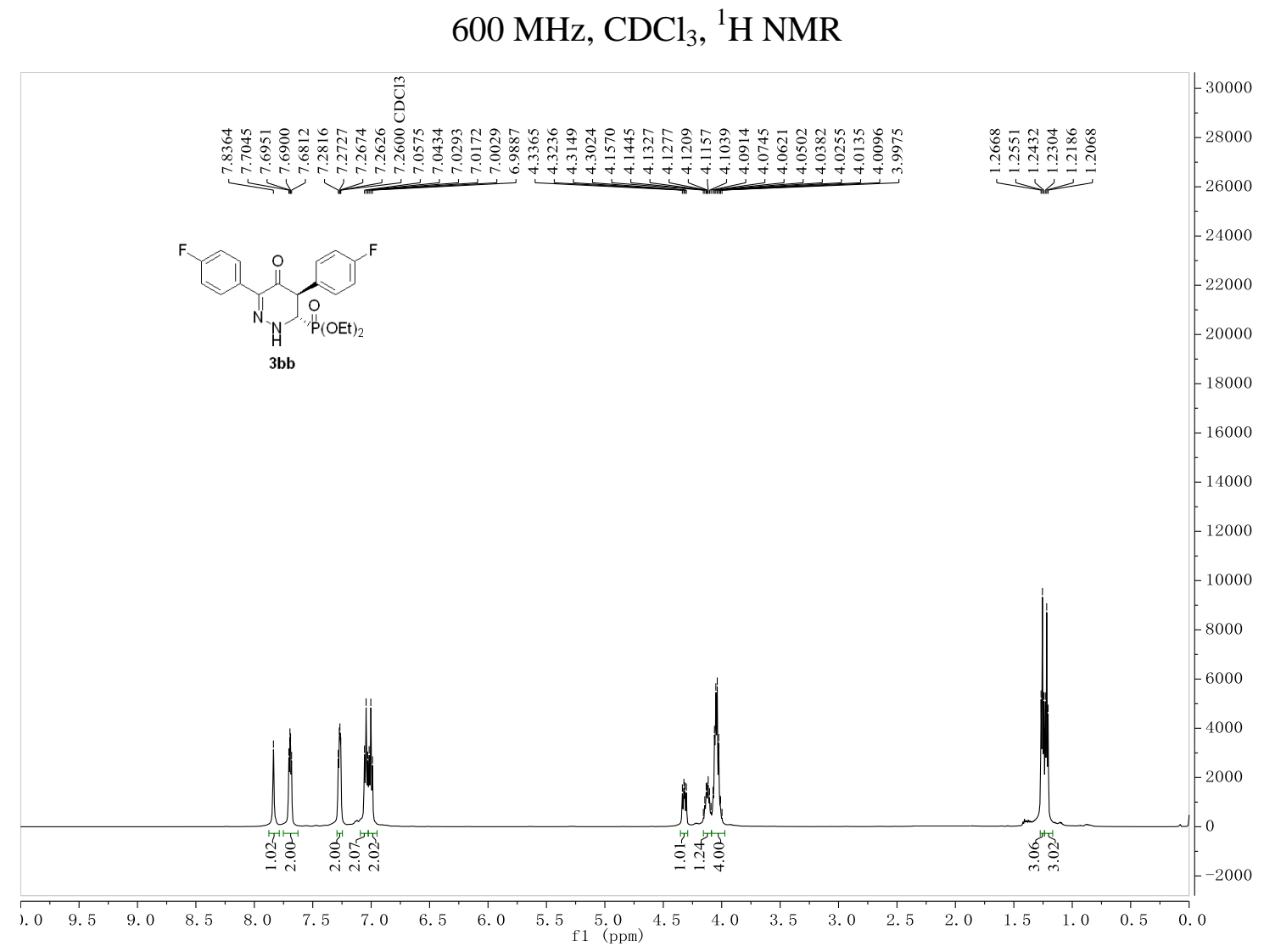

$151 \mathrm{MHz}, \mathrm{CDCl}_{3},{ }^{13} \mathrm{C}$ NMR

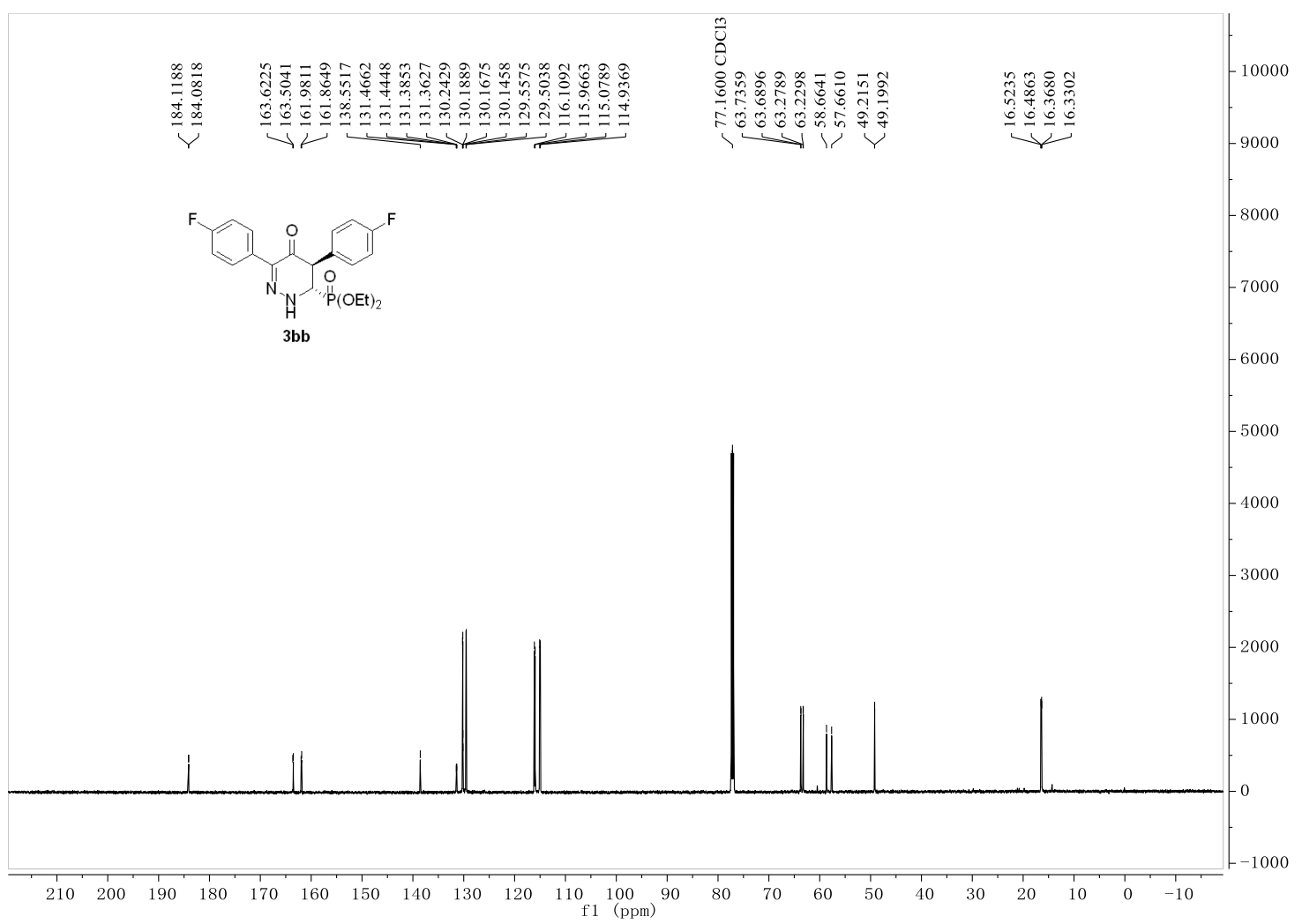




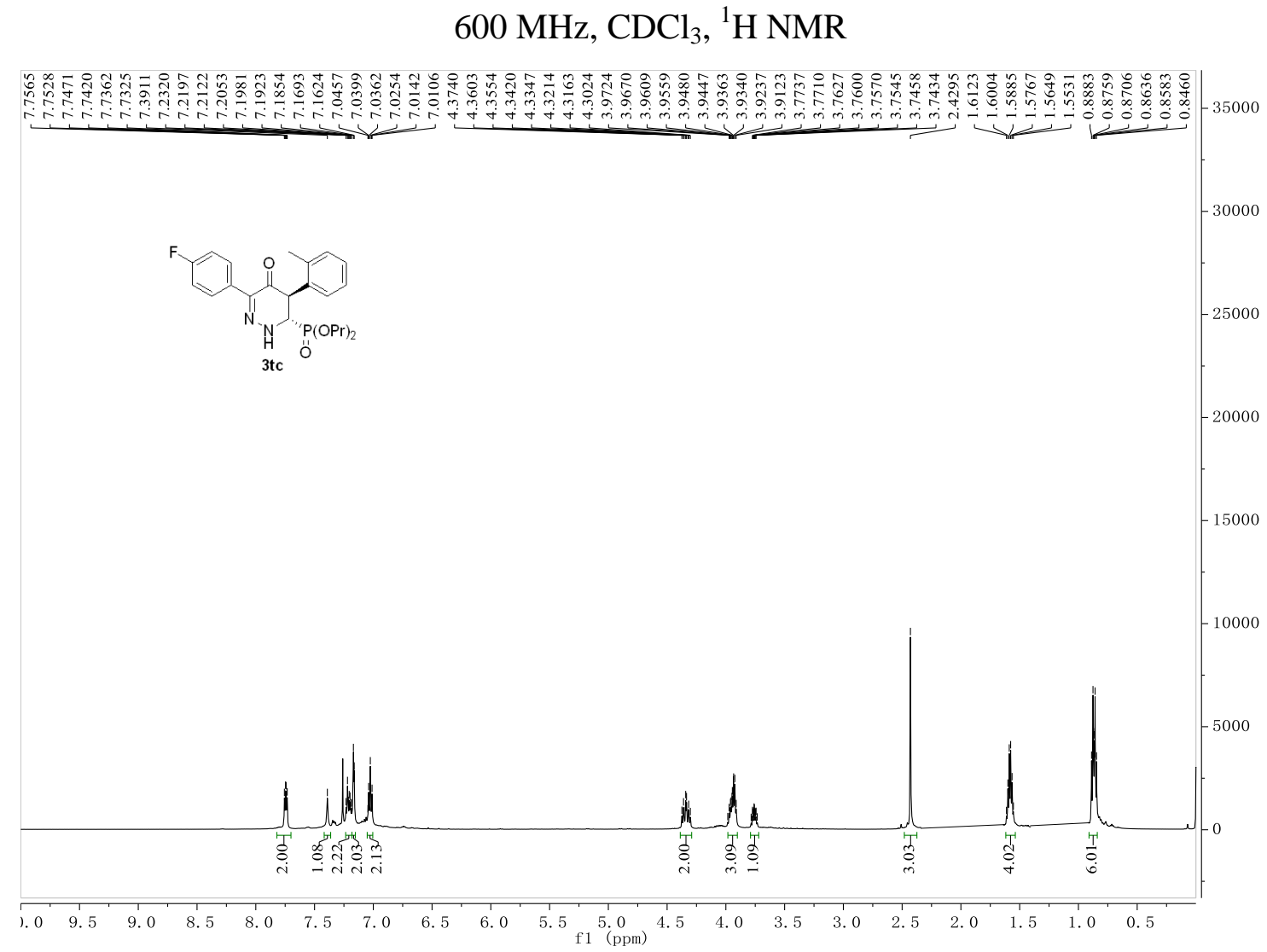

$151 \mathrm{MHz}, \mathrm{CDCl}_{3},{ }^{13} \mathrm{C}$ NMR

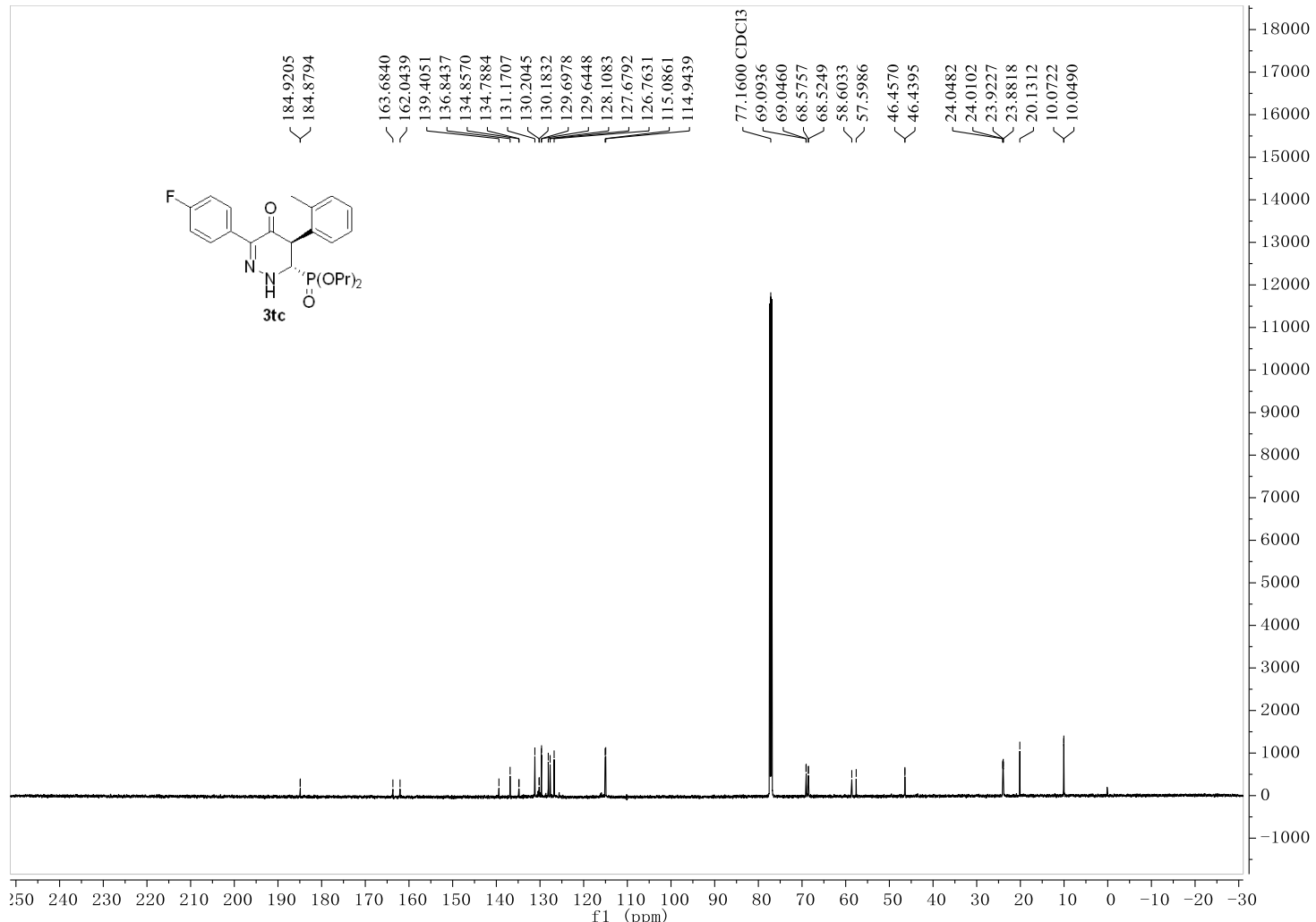


$600 \mathrm{MHz}, \mathrm{CDCl}_{3},{ }^{1} \mathrm{H} \mathrm{NMR}$

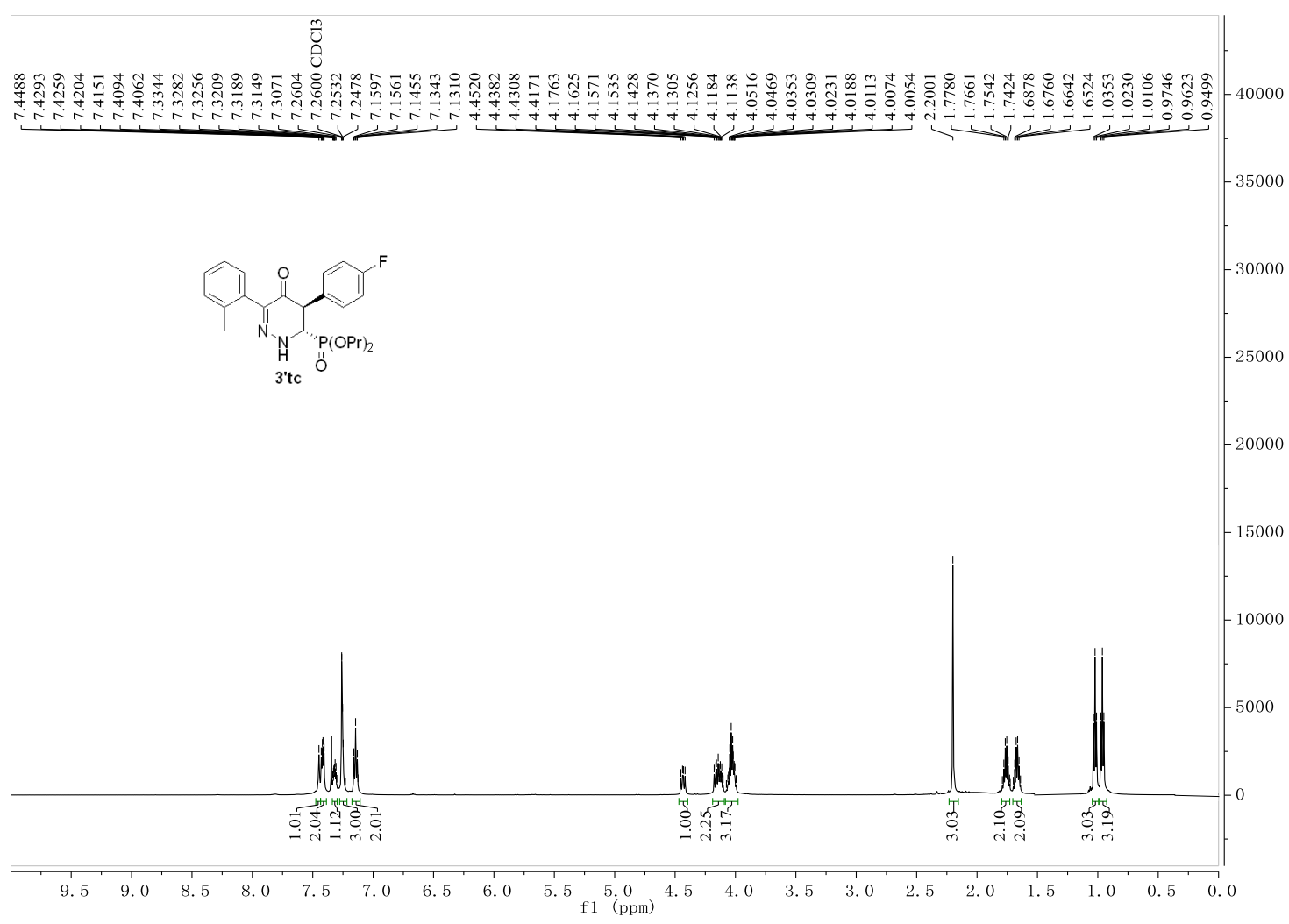

$151 \mathrm{MHz}, \mathrm{CDCl}_{3},{ }^{13} \mathrm{C} \mathrm{NMR}$

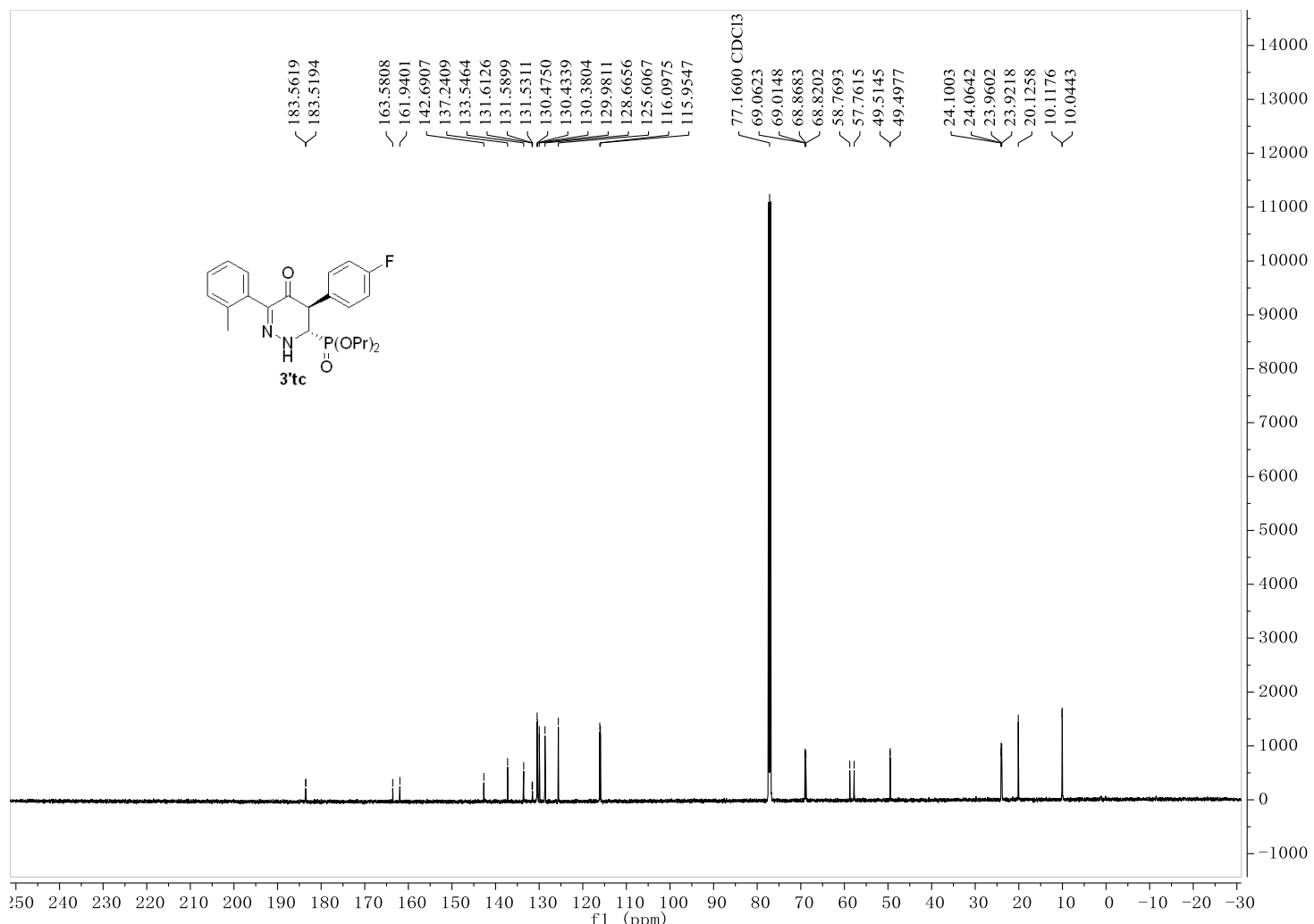




\section{$600 \mathrm{MHz}$, Acetone- $d_{6},{ }^{1} \mathrm{H}$ NMR}

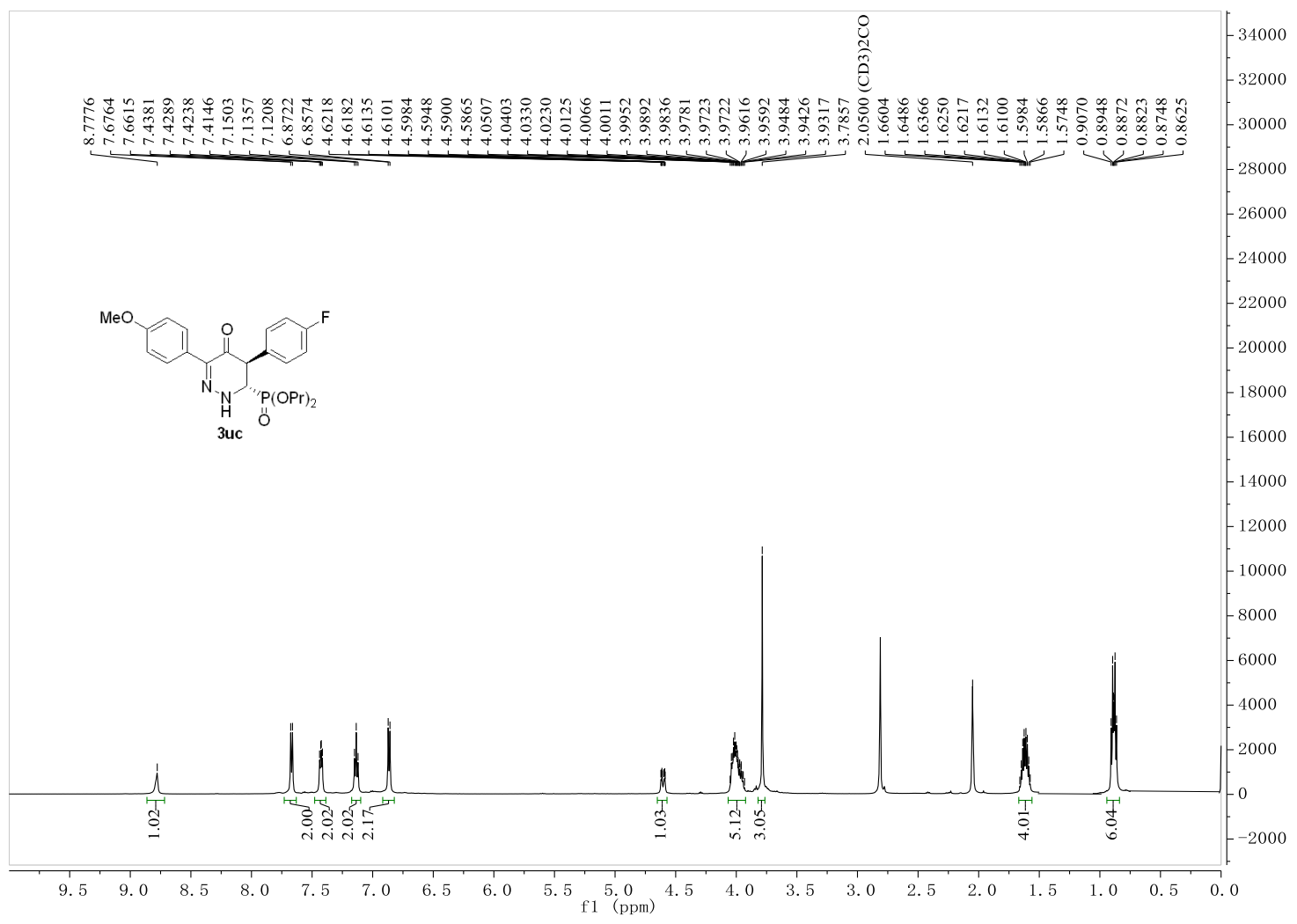

$151 \mathrm{MHz}$, Acetone- $d_{6},{ }^{13} \mathrm{C}$ NMR

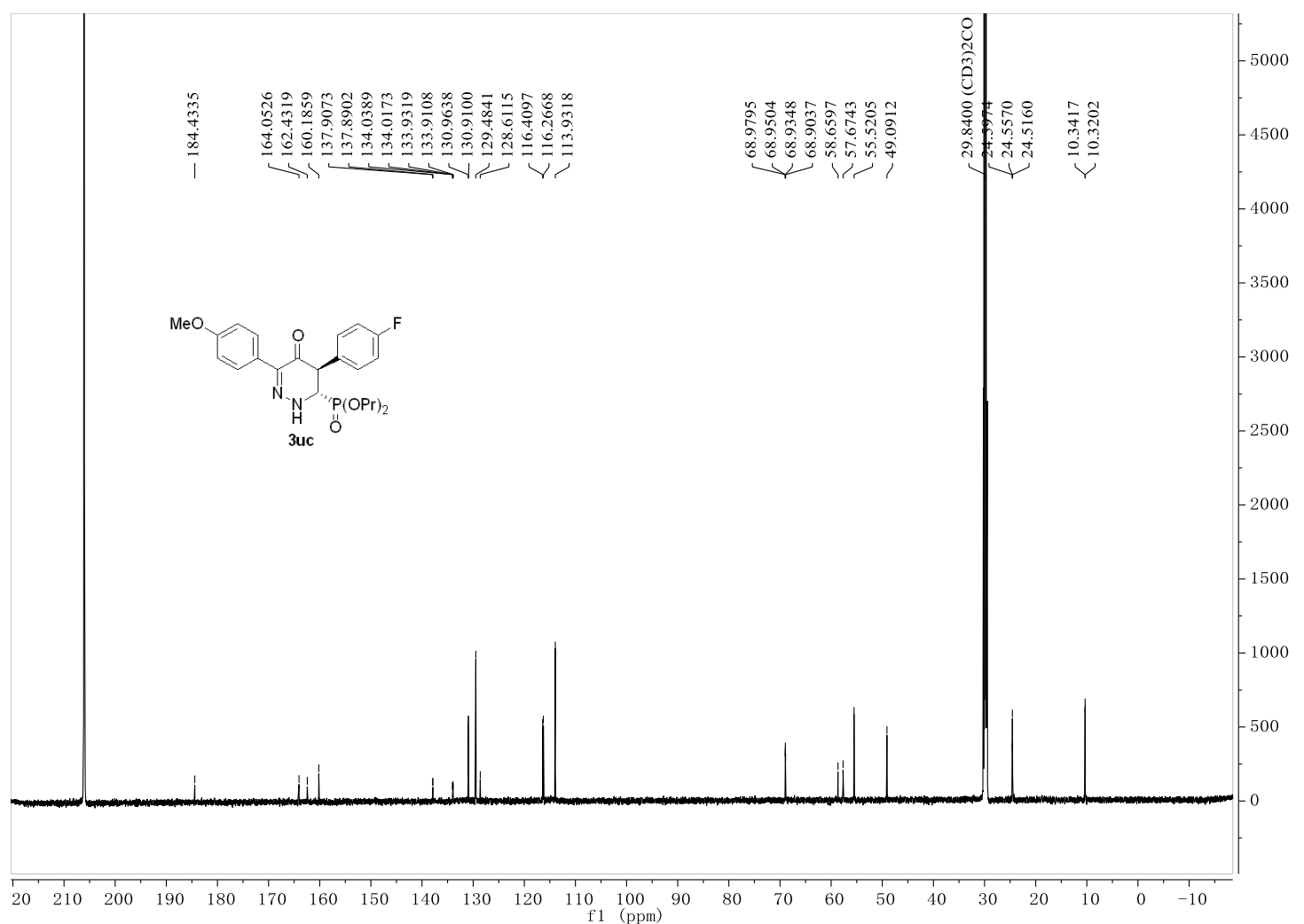


$600 \mathrm{MHz}$, Acetone- $d_{6},{ }^{1} \mathrm{H}$ NMR

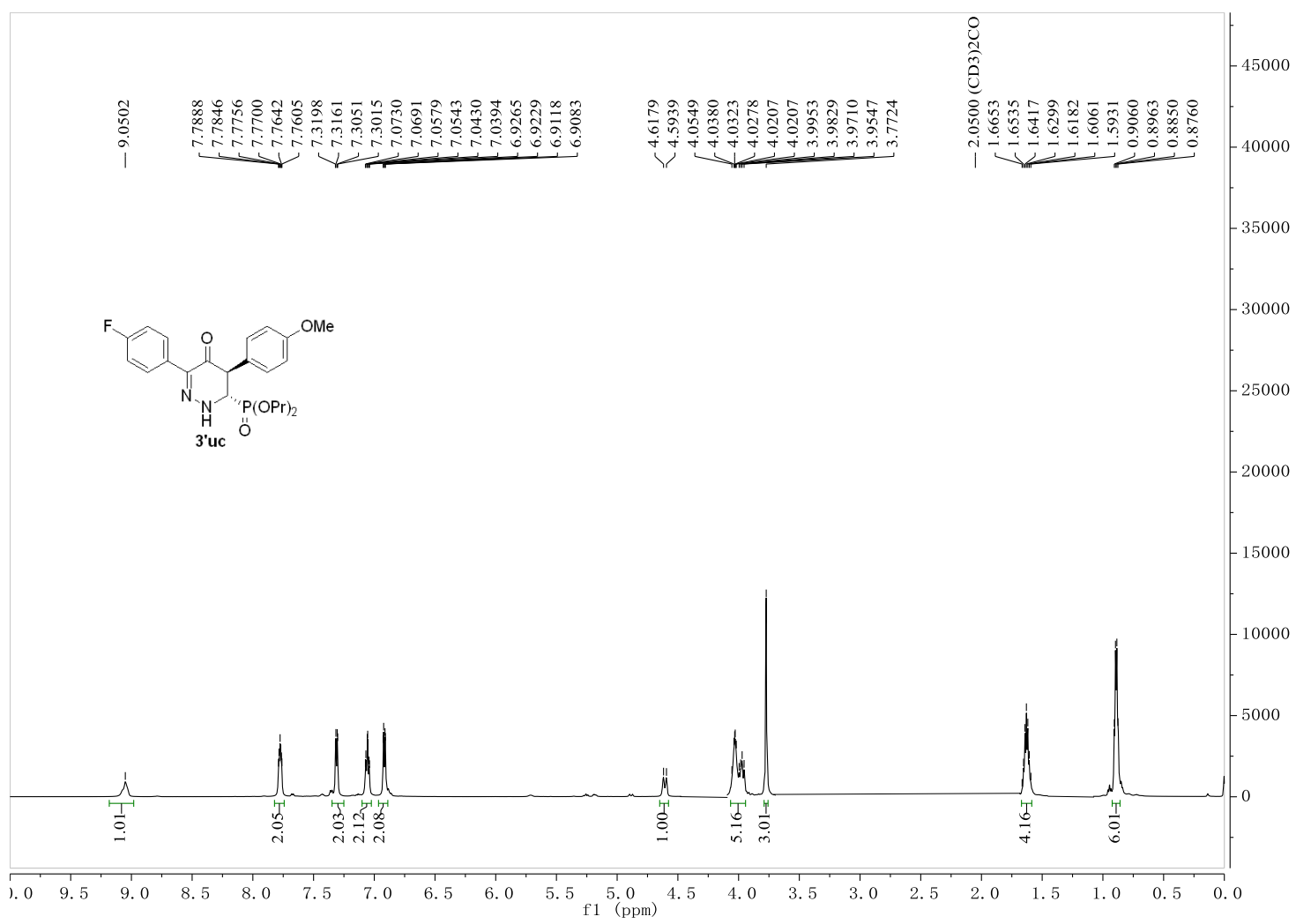

$151 \mathrm{MHz}$, Acetone- $d_{6},{ }^{13} \mathrm{C}$ NMR

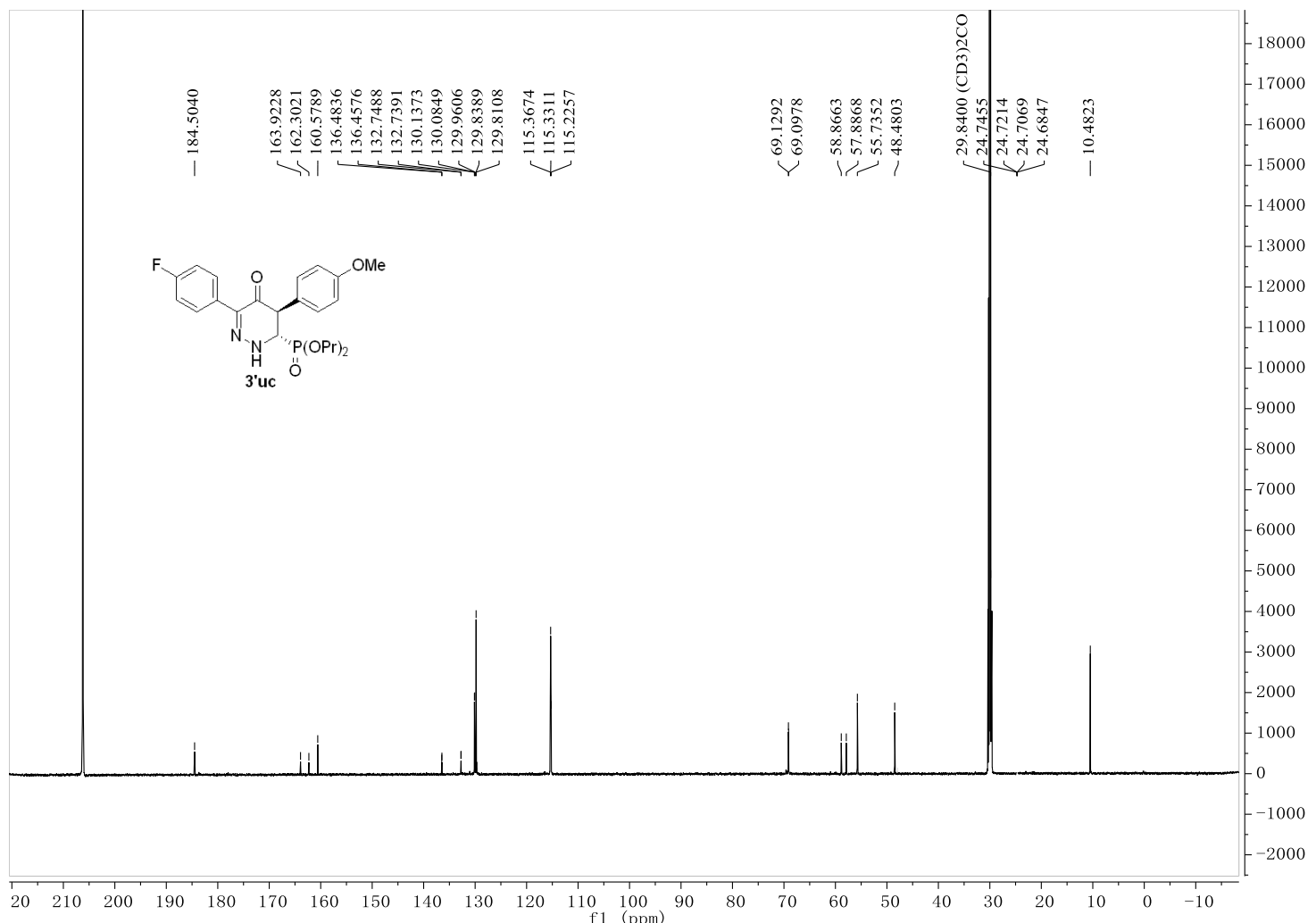




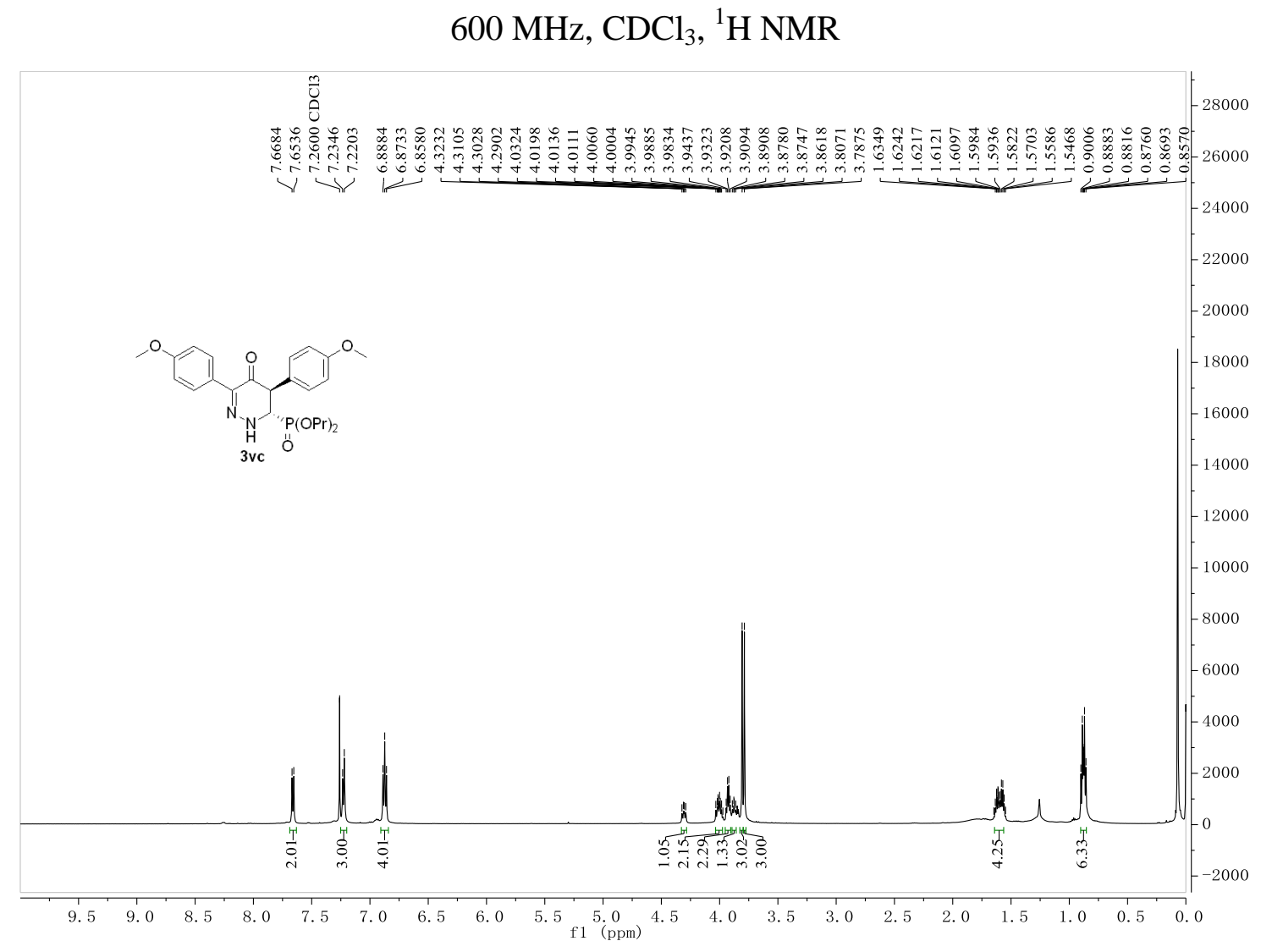

$151 \mathrm{MHz}, \mathrm{CDCl}_{3},{ }^{13} \mathrm{C} \mathrm{NMR}$

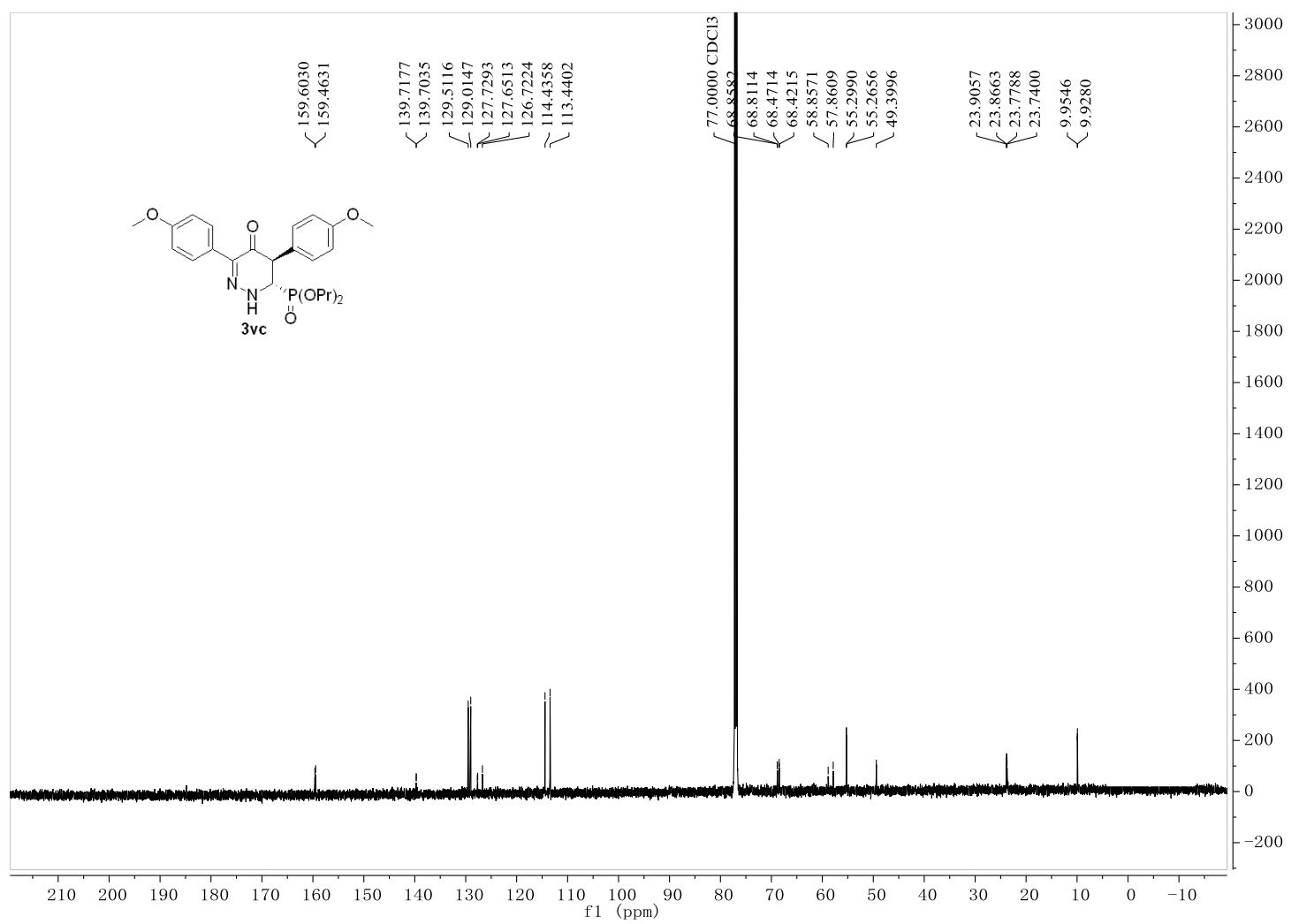


$600 \mathrm{MHz}, \mathrm{CDCl}_{3},{ }^{1} \mathrm{H} \mathrm{NMR}$

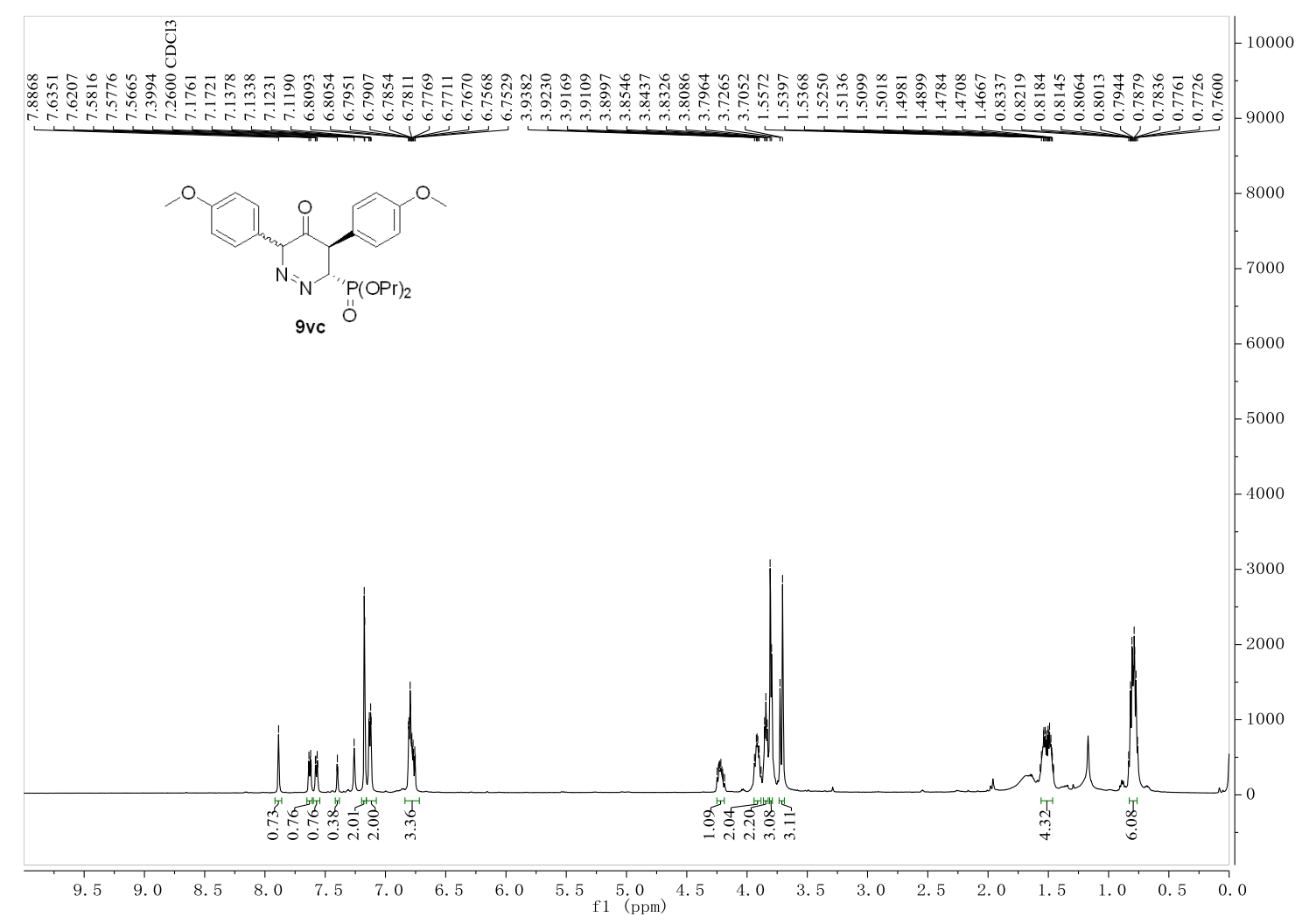

$151 \mathrm{MHz}, \mathrm{CDCl}_{3},{ }^{13} \mathrm{C} \mathrm{NMR}$

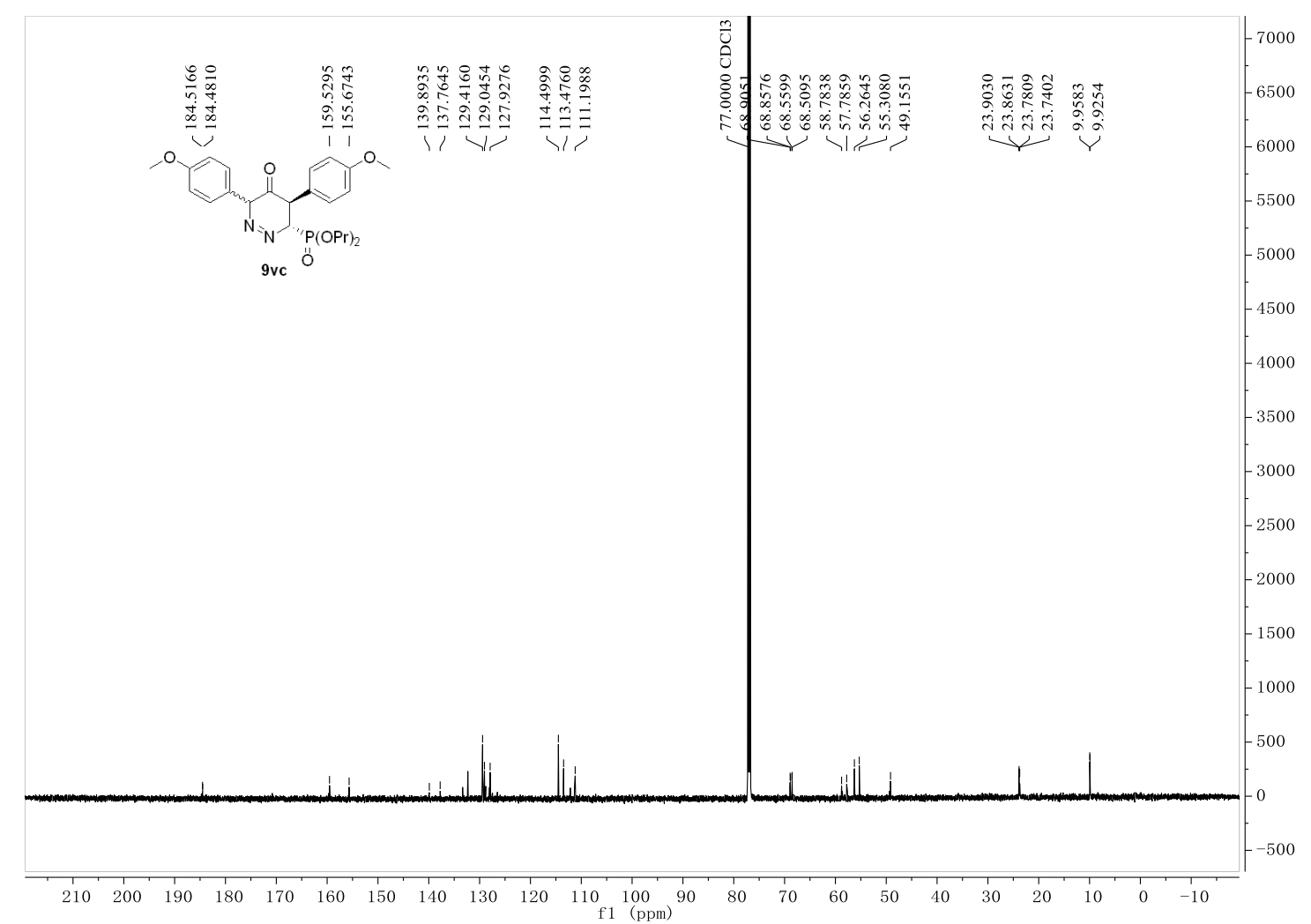




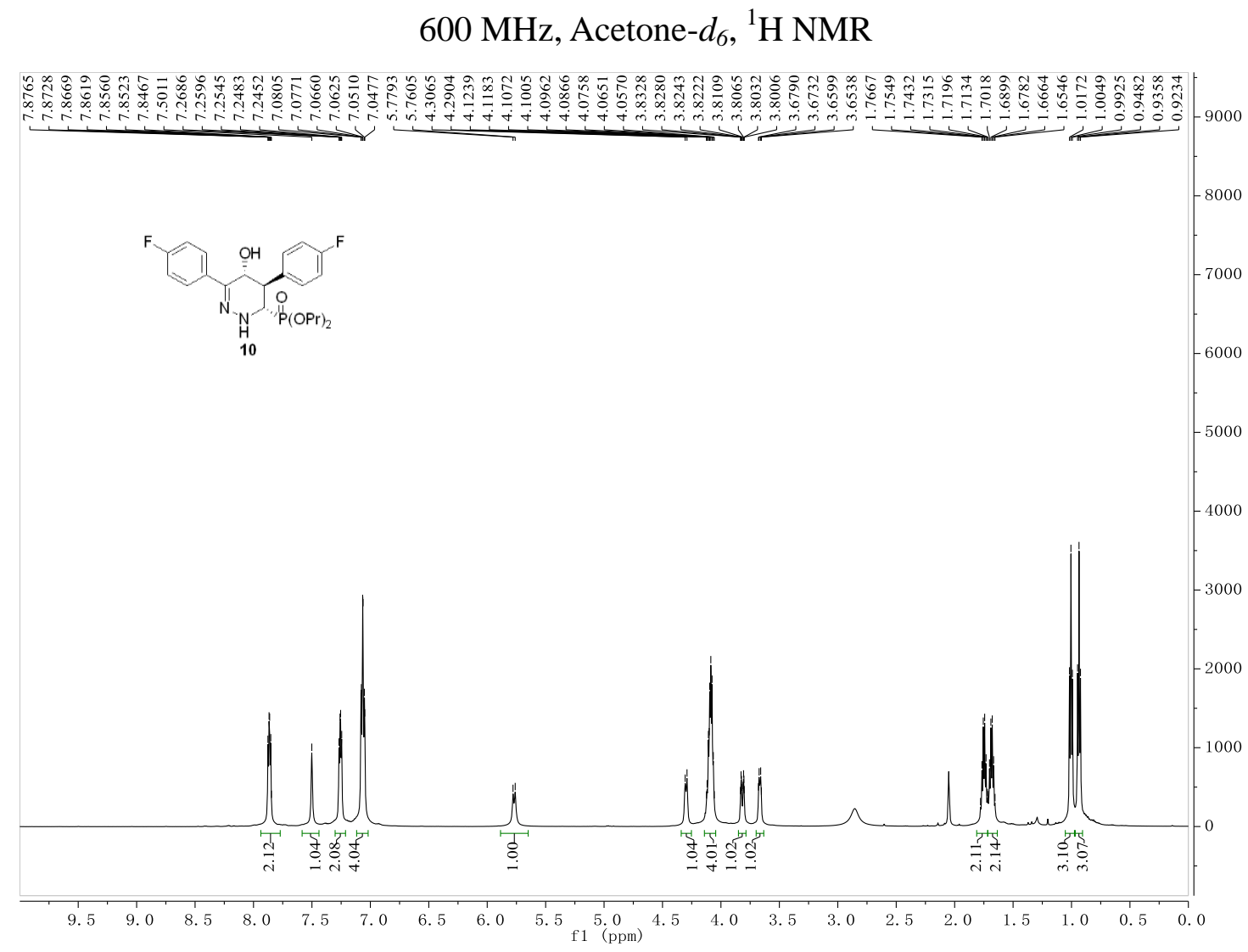

$151 \mathrm{MHz}$, Acetone- $d_{6},{ }^{13} \mathrm{C}$ NMR

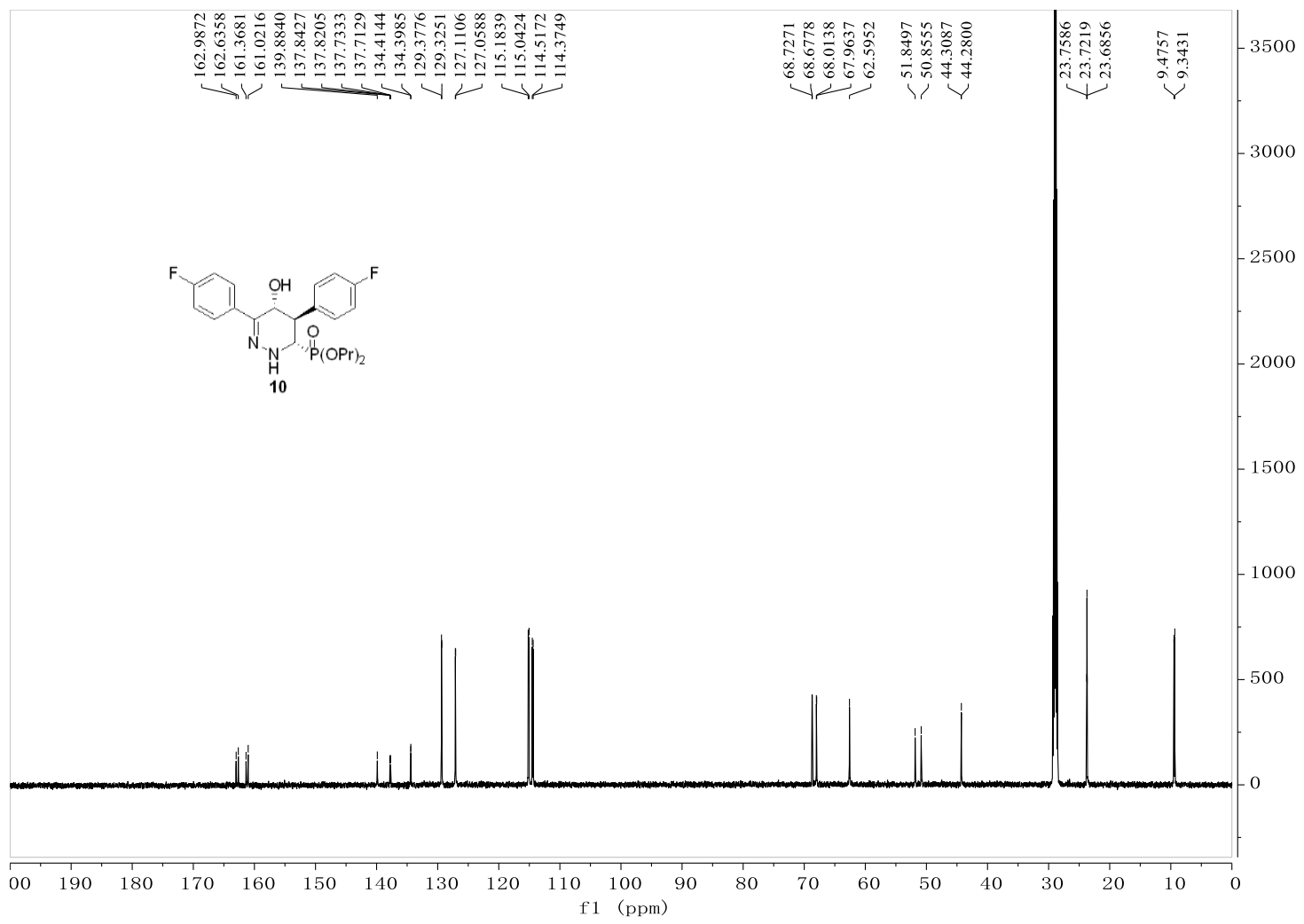




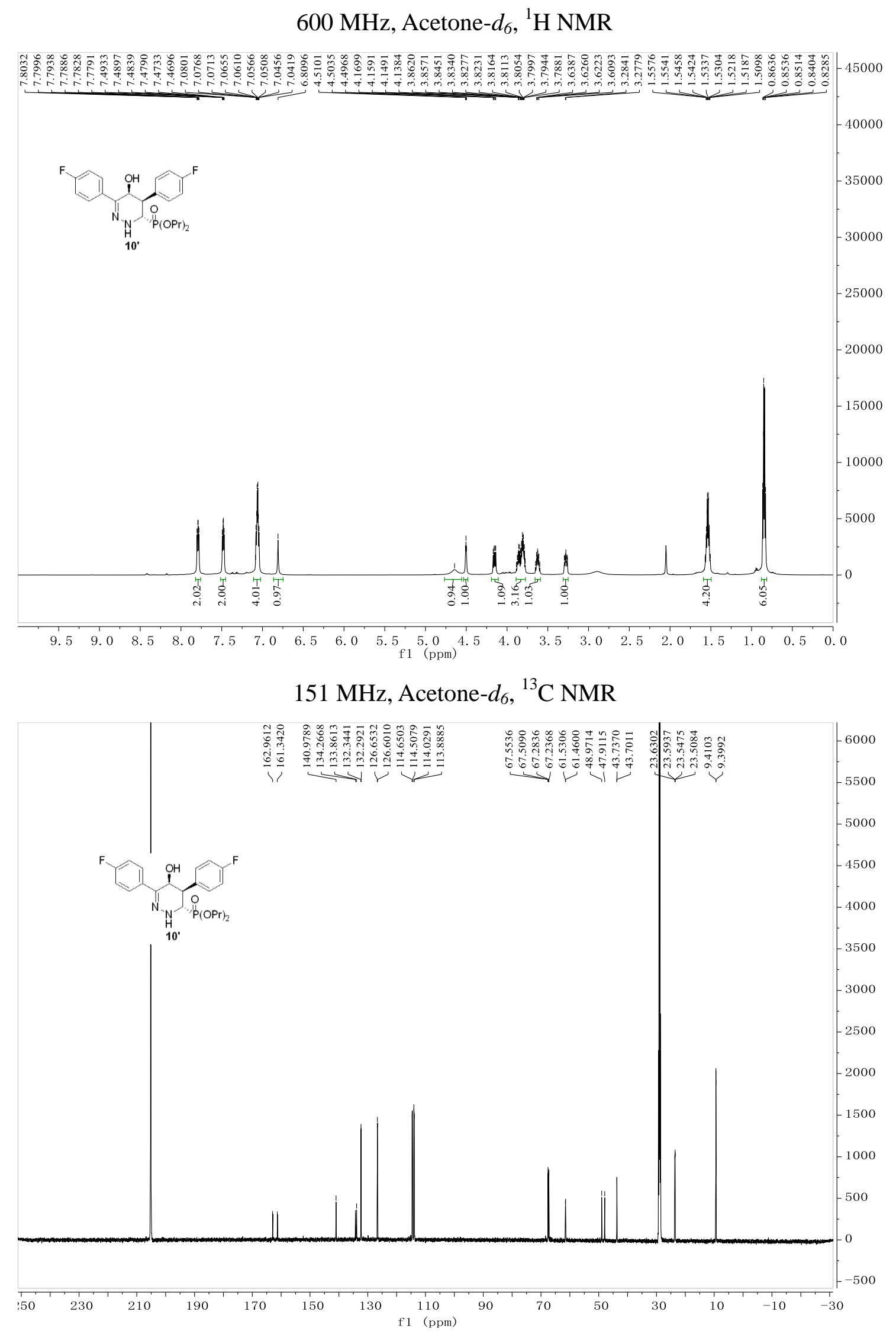


NOE (600 MHz, Acetone- $d_{6},{ }^{1} \mathrm{H}$ NMR; $600 \mathrm{MHz}$, Acetone- $d_{6},{ }^{1} \mathrm{H}$ NMR)

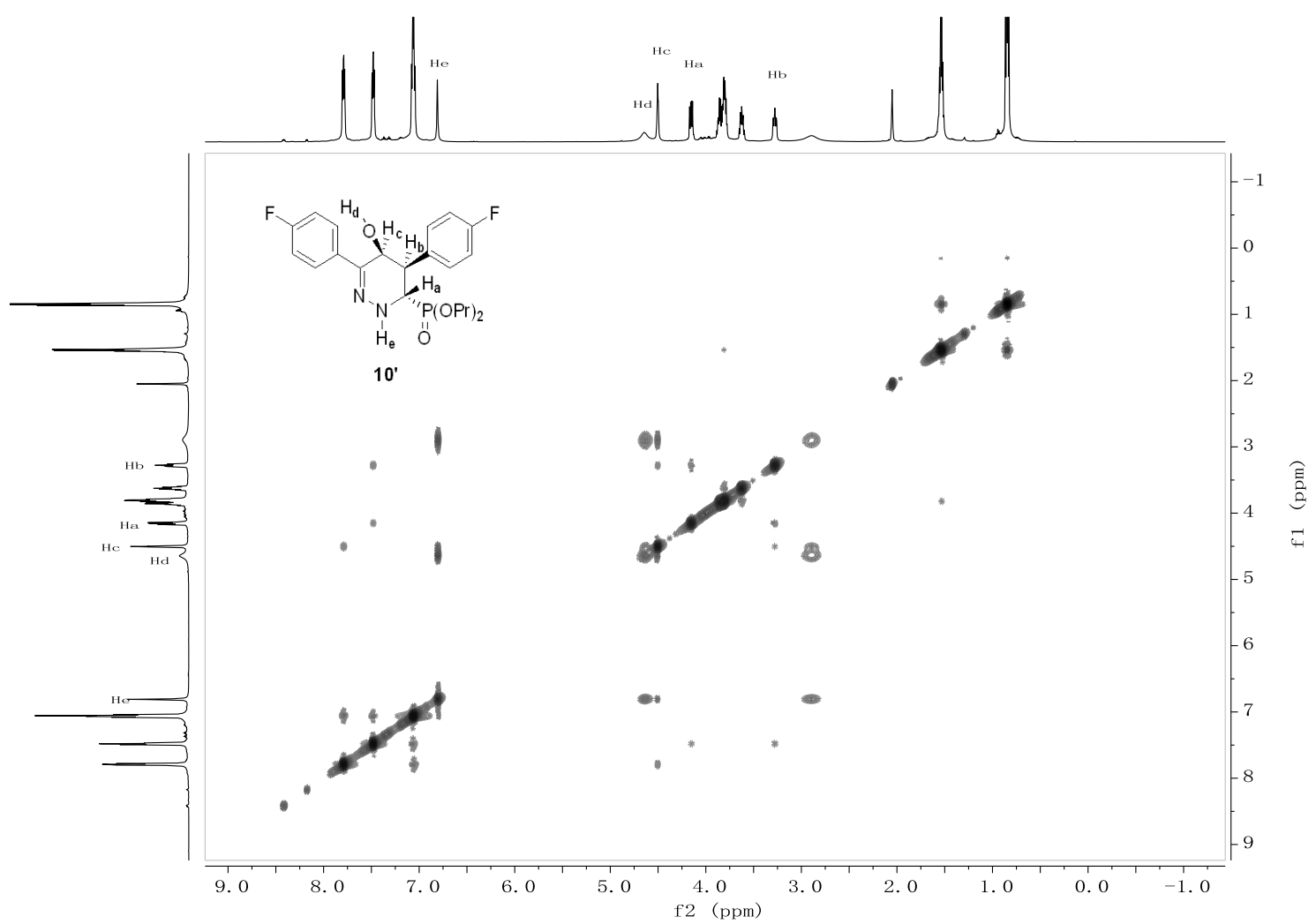

$600 \mathrm{MHz}$, Acetone- $d_{6},{ }^{1} \mathrm{H}$ NMR

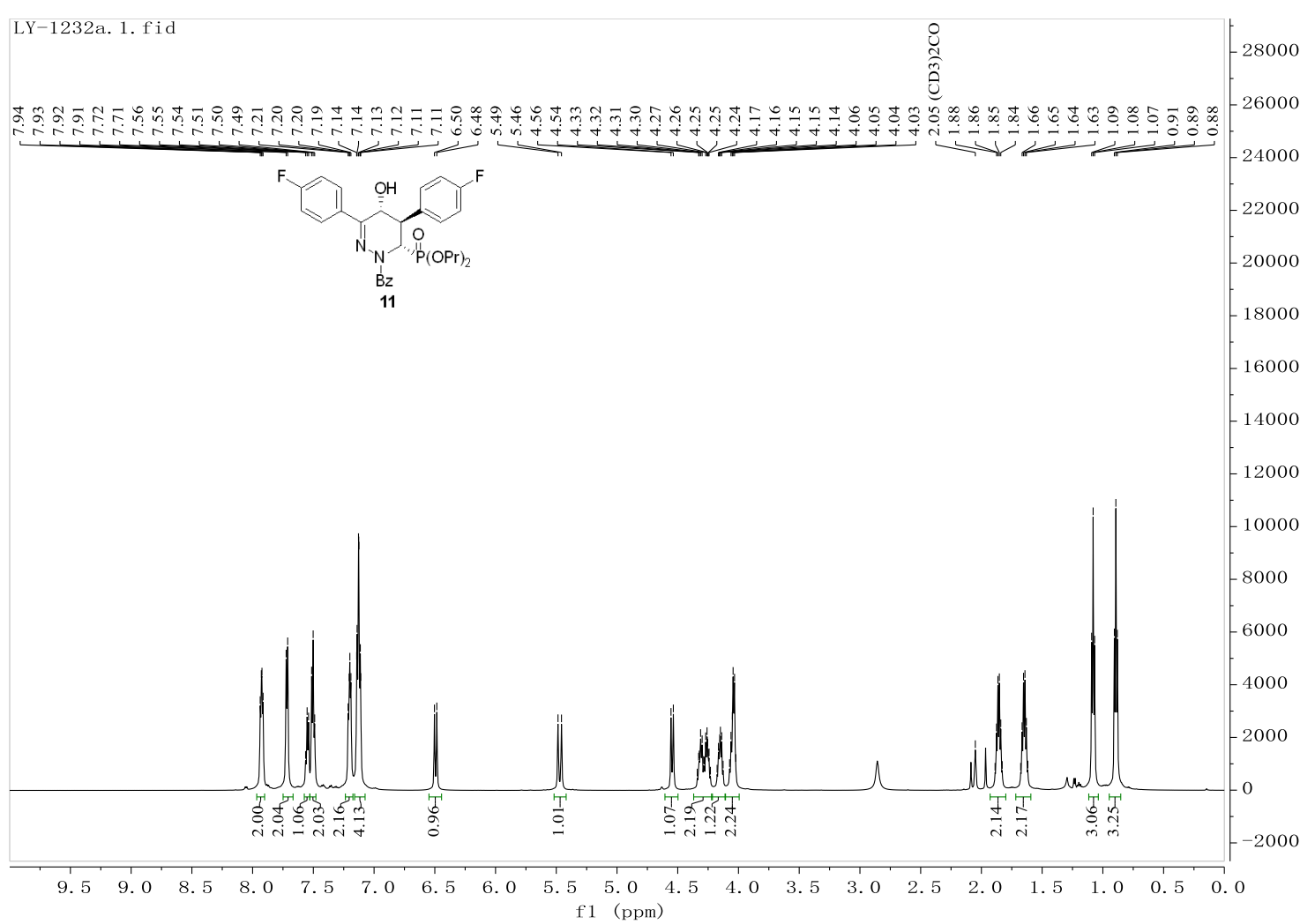


$151 \mathrm{MHz}$, Acetone- $d_{6},{ }^{13} \mathrm{C}$ NMR

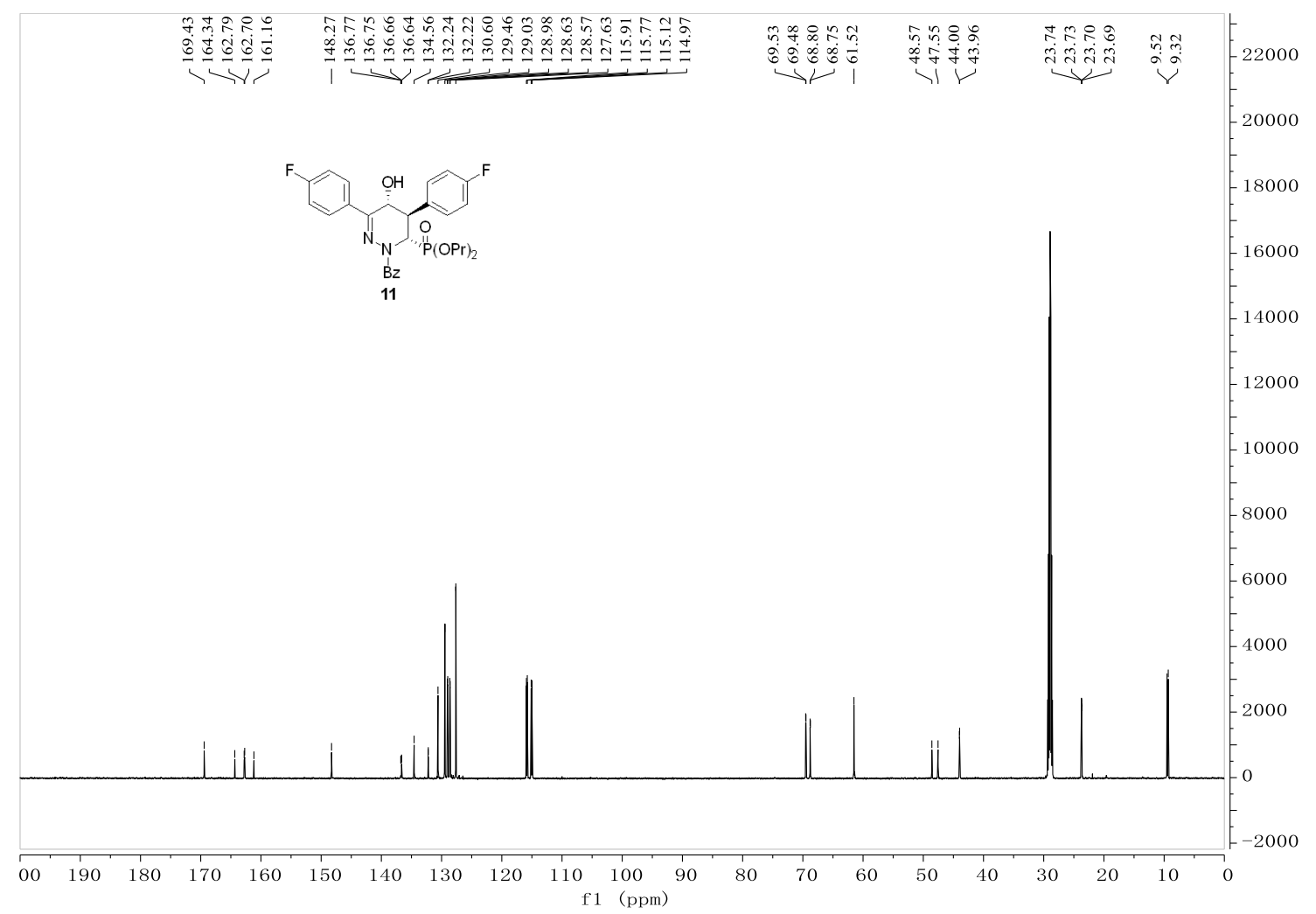

NOE (600 MHz, Acetone- $d_{6},{ }^{1} \mathrm{H}$ NMR; $600 \mathrm{MHz}$, Acetone- $d_{6},{ }^{1} \mathrm{H}$ NMR)

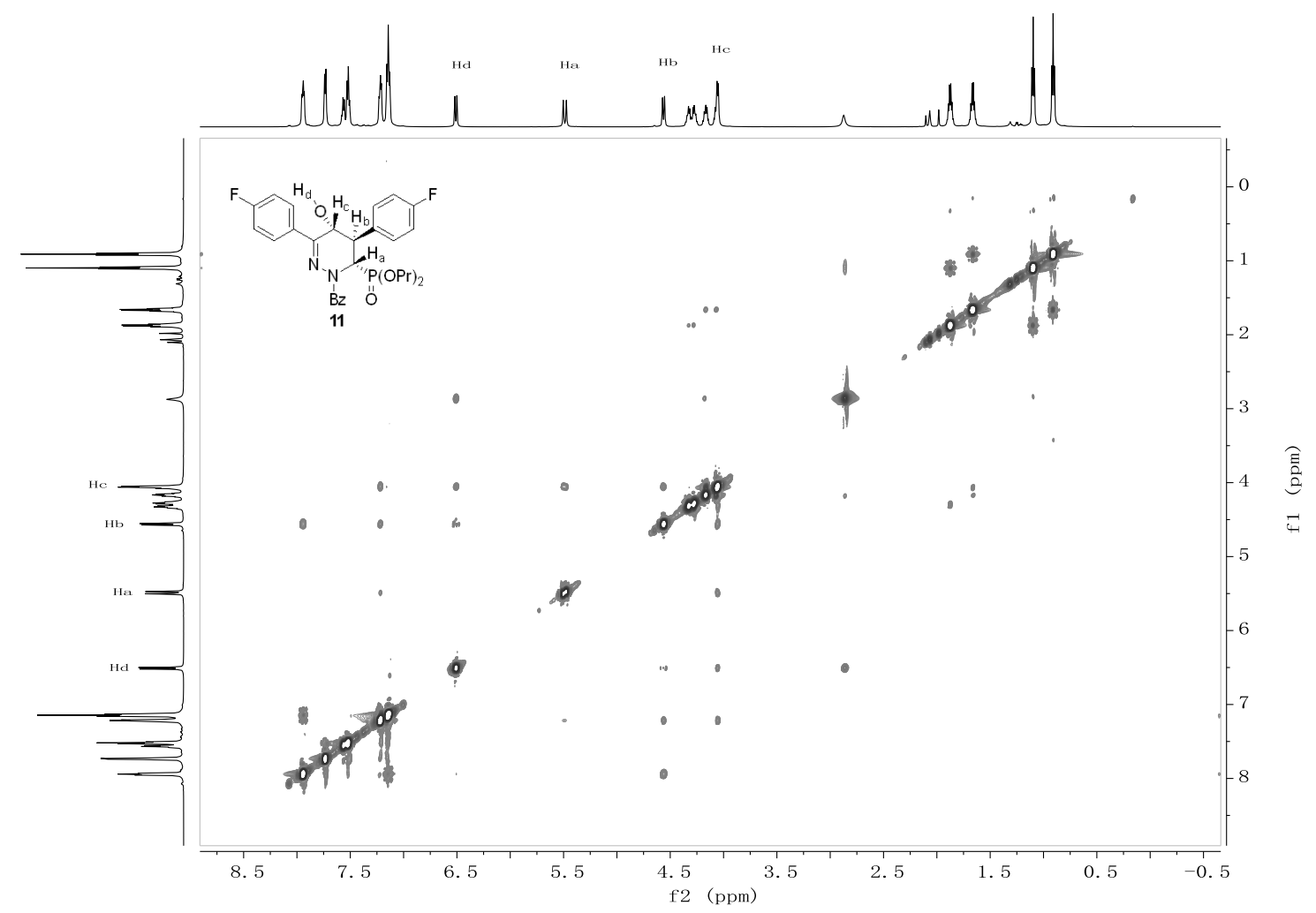




\section{$600 \mathrm{MHz}, \mathrm{CDCl}_{3},{ }^{1} \mathrm{H} \mathrm{NMR}$}

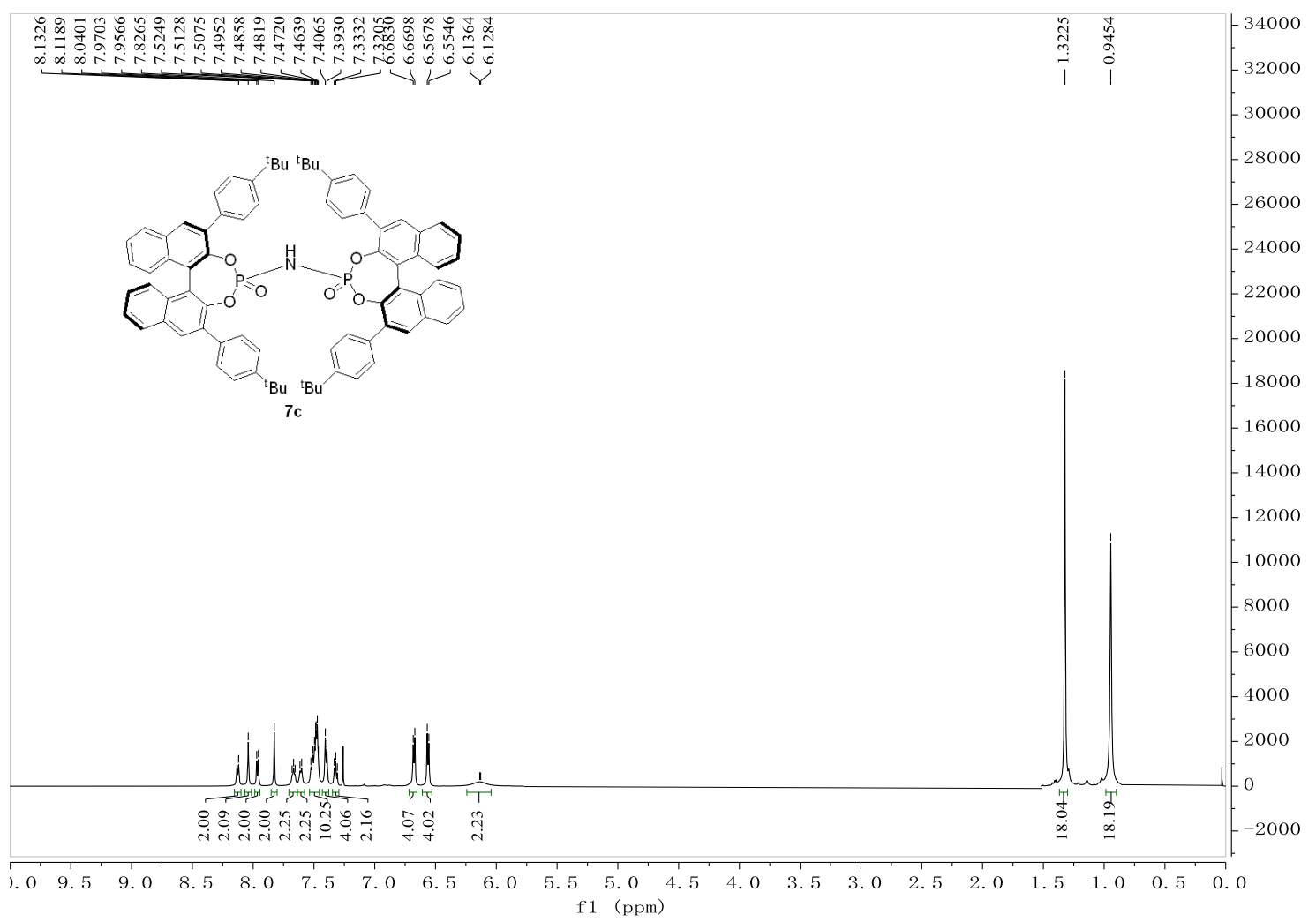

$600 \mathrm{MHz}, \mathrm{CDCl}_{3},{ }^{13} \mathrm{C} \mathrm{NMR}$

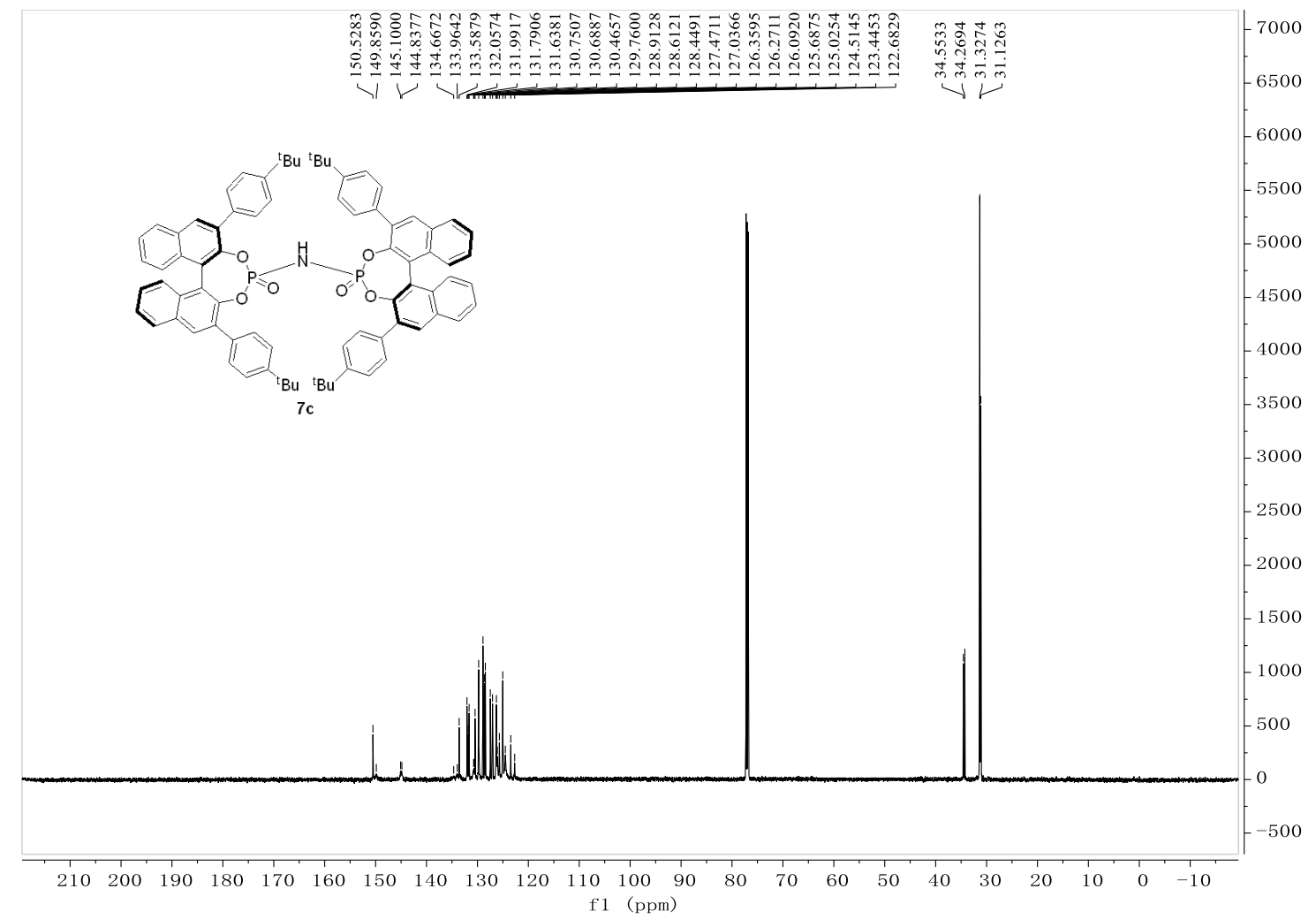




\section{The spectra of HPLC}<smiles>CO[P+]([O-])[C@@H]1NN=C(c2ccccc2)C(=O)[C@H]1c1ccccc1</smiles>

3aa

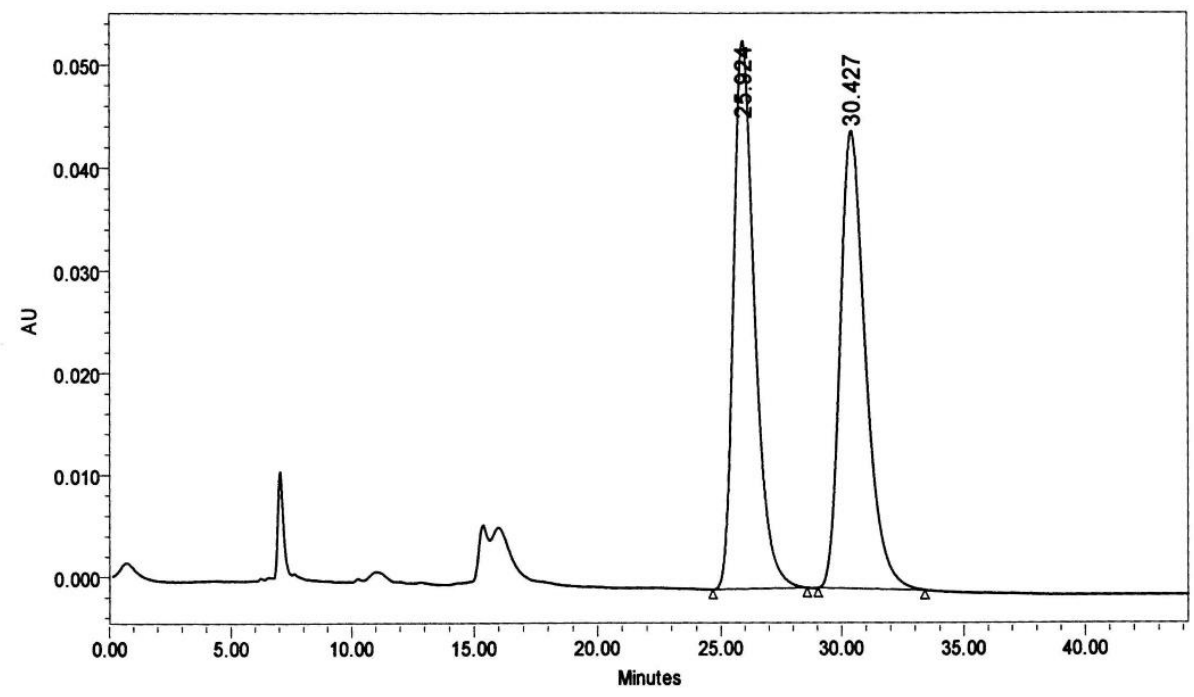

\begin{tabular}{|c|c|c|c|c|c|}
\hline & $\begin{array}{c}\text { RT } \\
(\mathrm{min})\end{array}$ & $\begin{array}{c}\text { Area } \\
\left(\mu \mathrm{V}^{*} \mathrm{sec}\right)\end{array}$ & $\%$ Area & $\begin{array}{c}\text { Height } \\
(\mu \mathrm{V})\end{array}$ & $\begin{array}{c}\% \\
\text { Height }\end{array}$ \\
\hline 1 & 25.924 & 3268679 & 50.07 & 53574 & 54.48 \\
\hline 2 & 30.427 & 3259824 & 49.93 & 44769 & 45.52 \\
\hline
\end{tabular}

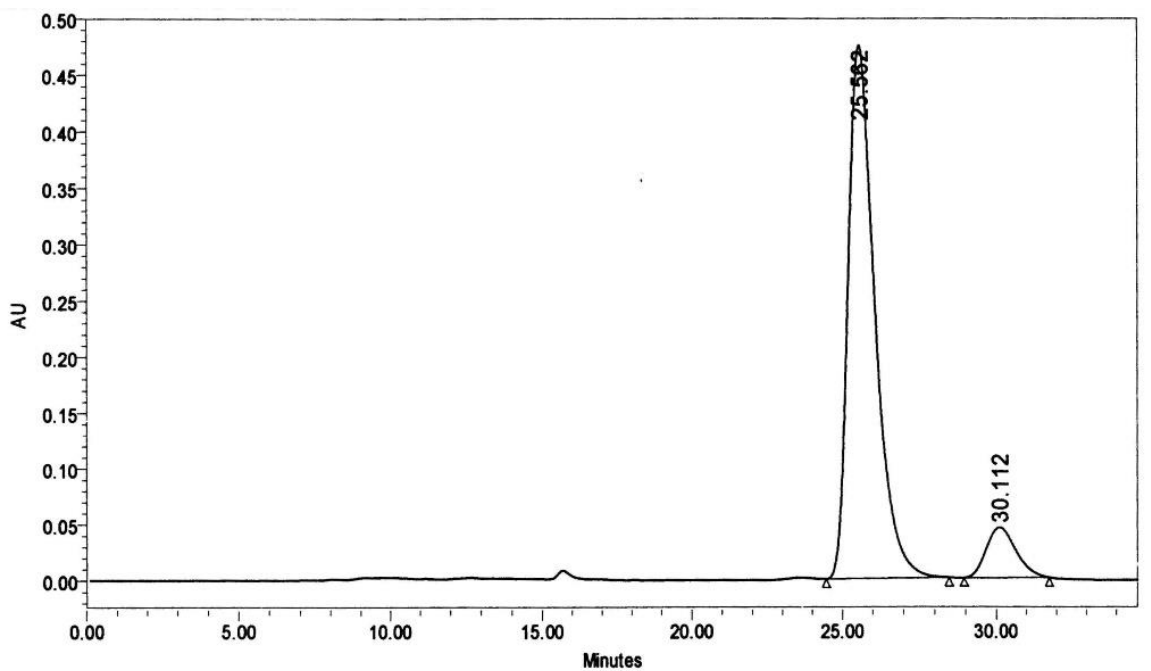

\begin{tabular}{|c|c|c|c|c|c|}
\hline & $\begin{array}{c}\mathrm{RT} \\
(\mathrm{min})\end{array}$ & $\begin{array}{c}\text { Area } \\
(\mu \mathrm{N} * \mathrm{sec})\end{array}$ & $\%$ Area & $\begin{array}{c}\text { Height } \\
(\mu \mathrm{V})\end{array}$ & $\begin{array}{c}\% \\
\text { Height }\end{array}$ \\
\hline 1 & 25.562 & 29028564 & 90.47 & 474834 & 91.35 \\
\hline 2 & 30.112 & 3057021 & 9.53 & 44962 & 8.65 \\
\hline
\end{tabular}


<smiles>CCOP(OCC)[C@@H]1NN=C(c2ccccc2)C(=O)C1c1ccccc1</smiles>

$3 a b$

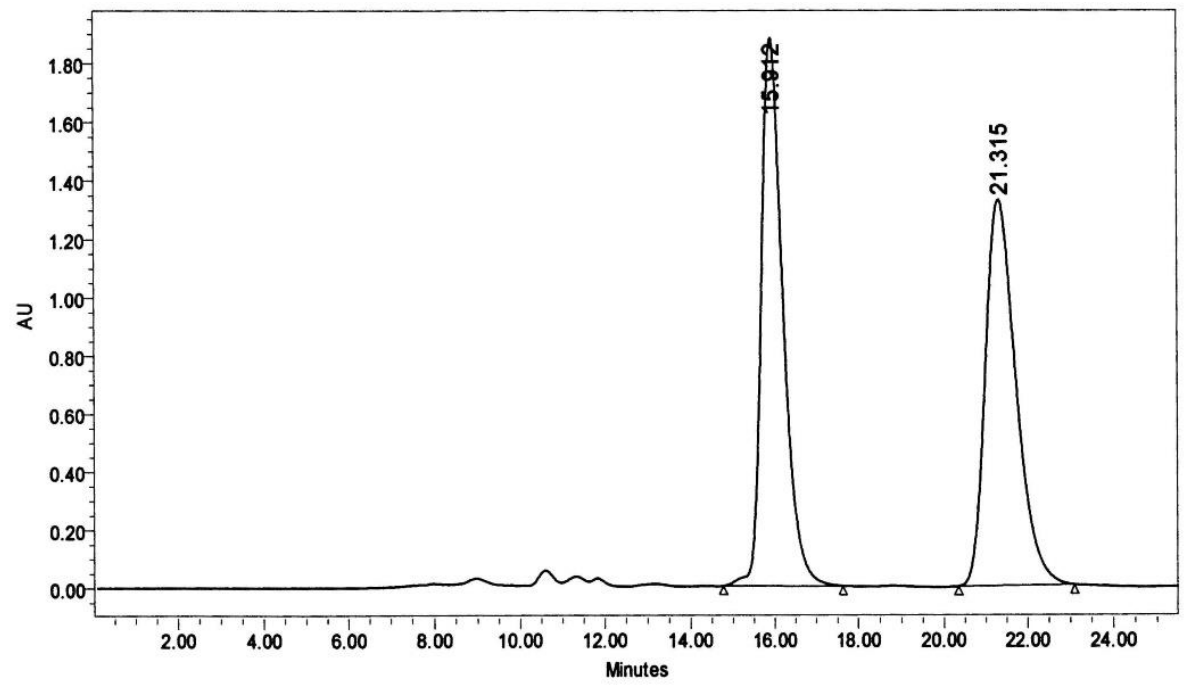

\begin{tabular}{|c|c|c|c|c|c|}
\hline & $\begin{array}{c}\mathrm{RT} \\
(\mathrm{min})\end{array}$ & $\begin{array}{c}\text { Area } \\
(\mu \mathrm{V} \text { "sec })\end{array}$ & $\%$ Area & $\begin{array}{c}\text { Height } \\
(\mu \mathrm{V})\end{array}$ & $\begin{array}{c}\% \\
\text { Height }\end{array}$ \\
\hline 1 & 15.912 & 68450975 & 50.35 & 1880346 & 58.61 \\
\hline 2 & 21.315 & 67509955 & 49.65 & 1327766 & 41.39 \\
\hline
\end{tabular}

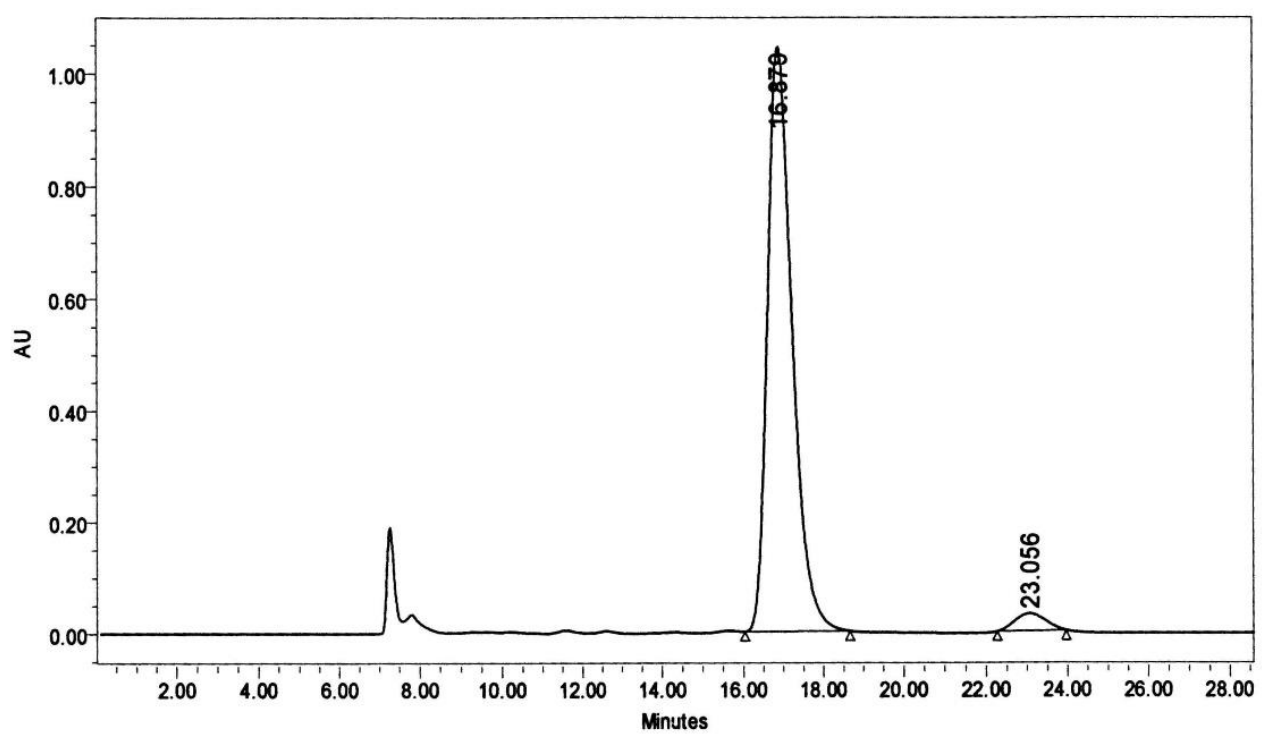

\begin{tabular}{|c|c|c|c|c|c|}
\hline & $\begin{array}{c}\mathrm{RT} \\
(\mathrm{min})\end{array}$ & $\begin{array}{c}\text { Area } \\
\left(\mathrm{NV}^{*} \mathrm{sec}\right)\end{array}$ & $\%$ Area & $\begin{array}{c}\text { Height } \\
(\mu \mathrm{V})\end{array}$ & $\begin{array}{c}\% \\
\text { Height }\end{array}$ \\
\hline 1 & 16.879 & 44723447 & 96.53 & 1043341 & 97.13 \\
\hline 2 & 23.056 & 1606648 & 3.47 & 30824 & 2.87 \\
\hline
\end{tabular}


<smiles>CCCCOP(C1NN=C(c2ccccc2)C(=O)C1c1ccccc1)[P+](O)c1ccccc1</smiles>

3ac

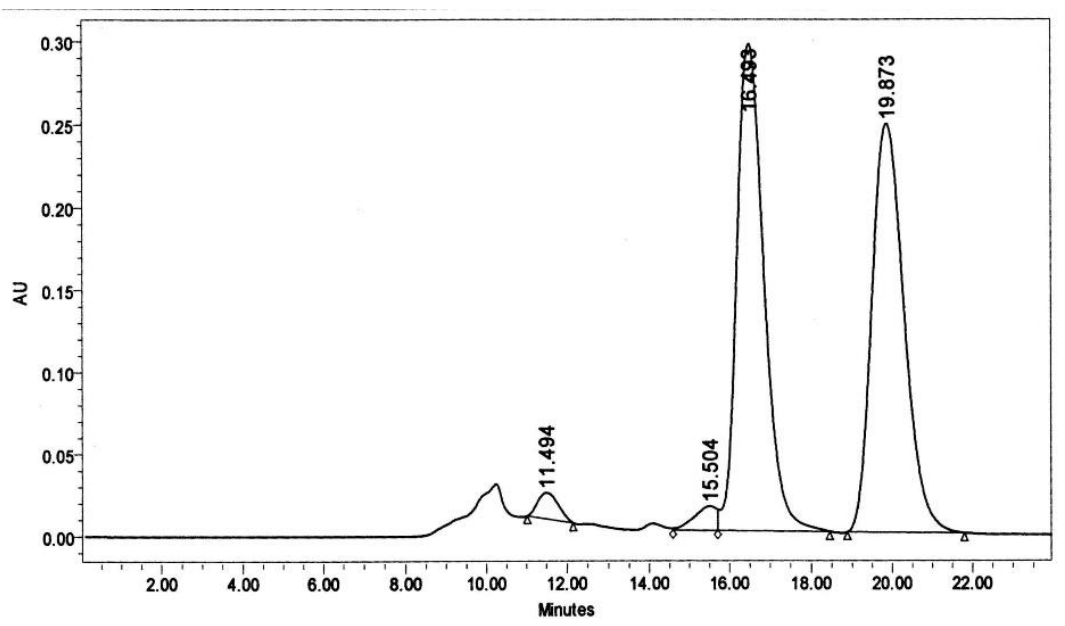

\begin{tabular}{|r|c|r|r|r|r|}
\hline & $\begin{array}{c}\mathrm{RT} \\
(\mathrm{min})\end{array}$ & $\begin{array}{c}\text { Area } \\
(\mu \mathrm{V} \text { ”sec })\end{array}$ & $\%$ Area & $\begin{array}{c}\text { Height } \\
(\mu \mathrm{V})\end{array}$ & $\begin{array}{c}\% \\
\text { Height }\end{array}$ \\
\hline 1 & 11.494 & 566639 & 1.96 & 15878 & 2.76 \\
\hline 2 & 15.504 & 569544 & 1.97 & 14749 & 2.57 \\
\hline 3 & 16.493 & 13861927 & 48.01 & 295476 & 51.45 \\
\hline 4 & 19.873 & 13875751 & 48.06 & 248152 & 43.21 \\
\hline
\end{tabular}

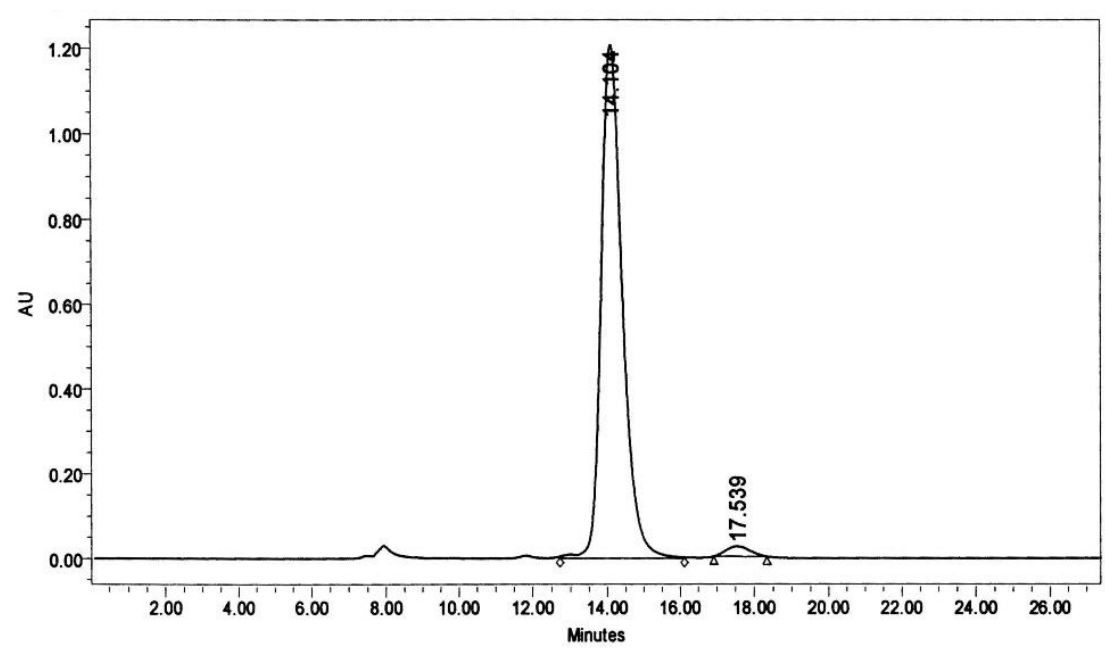

\begin{tabular}{|c|c|c|r|r|r|}
\hline & $\begin{array}{c}\mathrm{R} T \\
(\mathrm{~min})\end{array}$ & $\begin{array}{c}\text { Area } \\
(\mu \mathrm{V} * \mathrm{sec})\end{array}$ & $\%$ Area & $\begin{array}{c}\text { Height } \\
(\mu \mathrm{V})\end{array}$ & $\begin{array}{c}\% \\
\text { Height }\end{array}$ \\
\hline 1 & 14.104 & 48802921 & 97.92 & 1207092 & 98.07 \\
\hline 2 & 17.539 & 1038473 & 2.08 & 23699 & 1.93 \\
\hline
\end{tabular}




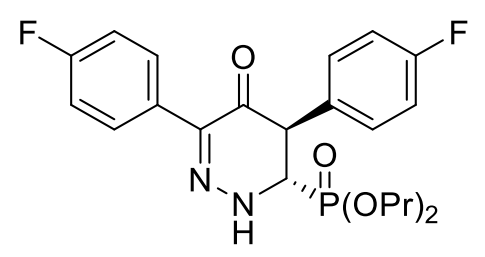

3bc

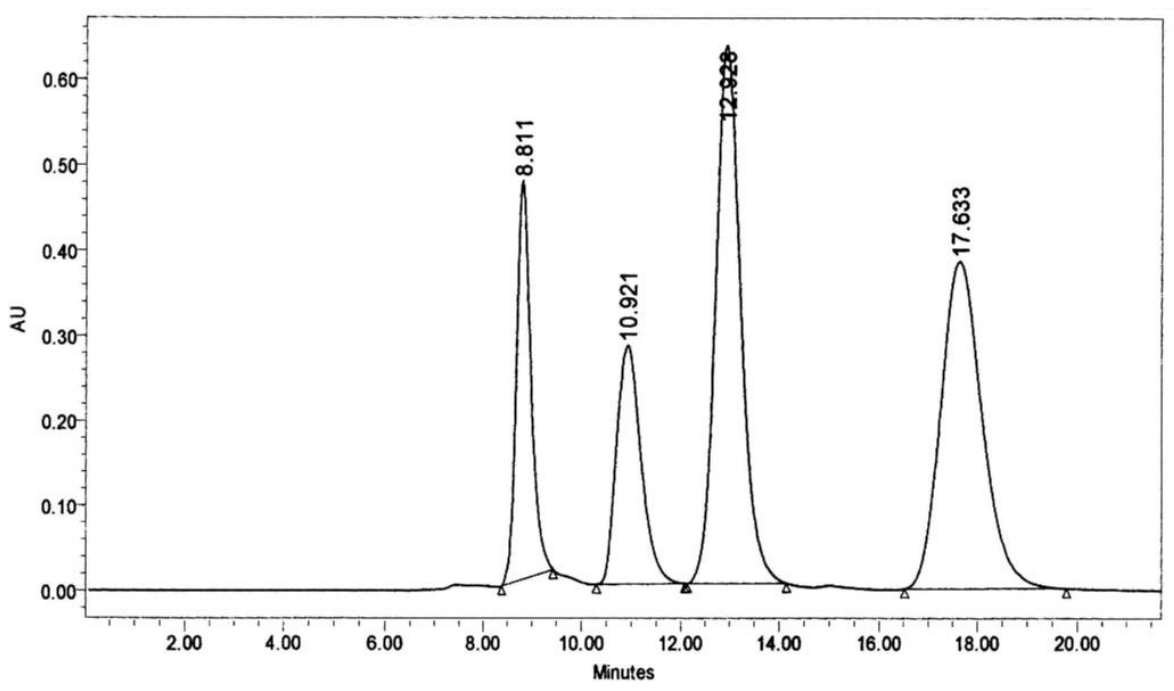

\begin{tabular}{|c|c|c|c|c|c|}
\hline & $\begin{array}{c}\mathrm{RT} \\
(\mathrm{min})\end{array}$ & $\begin{array}{c}\text { Area } \\
\left(\mu \mathrm{V}^{*} \mathrm{sec}\right)\end{array}$ & $\%$ Area & $\begin{array}{c}\text { Height } \\
(\mu \mathrm{V})\end{array}$ & $\begin{array}{c}\% \\
\text { Height }\end{array}$ \\
\hline 1 & 8.811 & 9729714 & 15.06 & 468917 & 26.55 \\
\hline 2 & 10.921 & 9639414 & 14.92 & 280829 & 15.90 \\
\hline 3 & 12.928 & 22683439 & 35.12 & 631166 & 35.74 \\
\hline 4 & 17.633 & 22540113 & 34.90 & 385104 & 21.81 \\
\hline
\end{tabular}

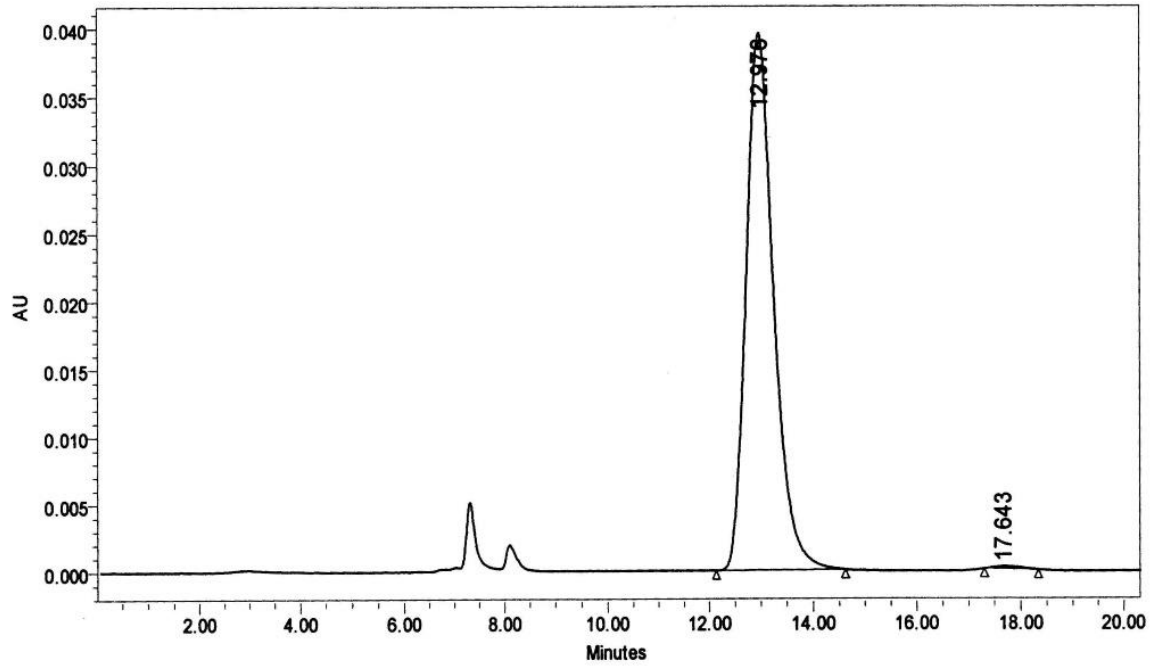

\begin{tabular}{|r|c|r|r|r|r|}
\hline & $\begin{array}{c}\mathrm{RT} \\
(\mathrm{min})\end{array}$ & $\begin{array}{c}\text { Area } \\
\left(\mu \mathrm{V}^{*} \mathrm{sec}\right)\end{array}$ & $\%$ Area & $\begin{array}{l}\text { Height } \\
(\mu \mathrm{V})\end{array}$ & $\begin{array}{c}\% \\
\text { Height }\end{array}$ \\
\hline 1 & 12.976 & 1465087 & 99.54 & 39550 & 99.50 \\
\hline 2 & 17.643 & 6803 & 0.46 & 199 & 0.50 \\
\hline
\end{tabular}


<smiles>O=C1C(c2ccc(Cl)cc2)=NNC(P(=O)(O)OCc2ccccc2)[C@@H]1c1ccc(Cl)cc1</smiles>

$3 c c$

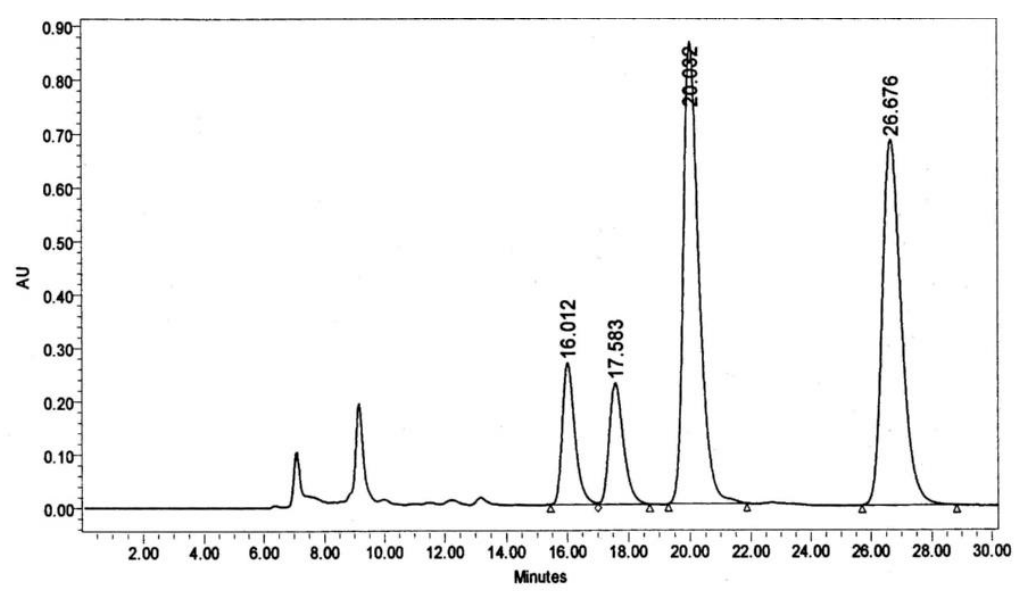

\begin{tabular}{|c|c|c|r|r|c|}
\hline & $\begin{array}{c}\text { RT } \\
(\mathrm{min})\end{array}$ & $\begin{array}{c}\text { Area } \\
(\mu \mathrm{V} \times \mathrm{sec})\end{array}$ & $\%$ Area & $\begin{array}{c}\text { Height } \\
(\mu \mathrm{V})\end{array}$ & $\begin{array}{c}\% \\
\text { Height }\end{array}$ \\
\hline 1 & 16.012 & 7667548 & 10.19 & 265071 & 12.99 \\
\hline 2 & 17.583 & 7323236 & 9.74 & 226948 & 11.12 \\
\hline 3 & 20.032 & 30368719 & 40.38 & 863865 & 42.34 \\
\hline 4 & 26.676 & 29852075 & 39.69 & 684452 & 33.55 \\
\hline
\end{tabular}

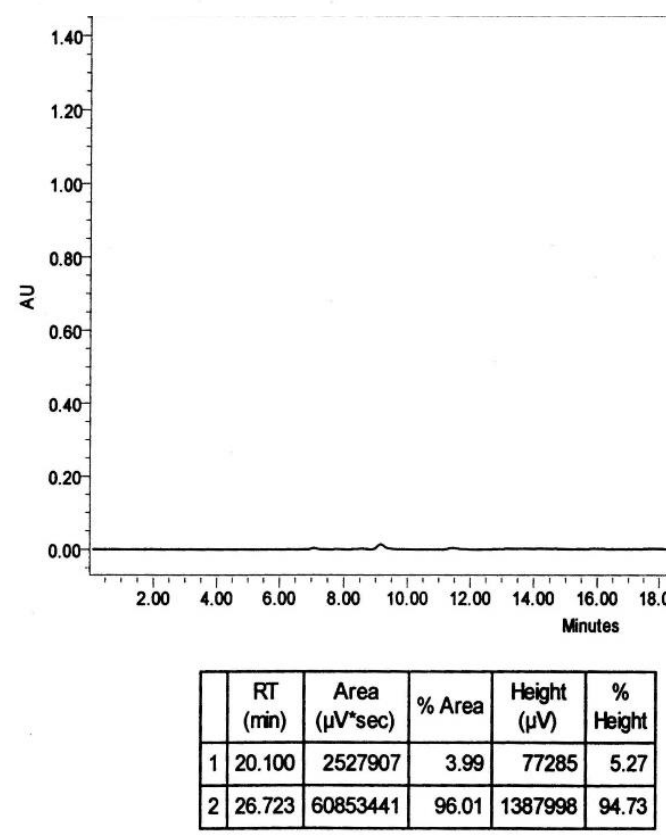


<smiles>CCOC(=O)[C@@H]1NN=C(c2ccc(Br)cc2)C(=O)[C@@H]1c1ccc(Br)cc1</smiles>

$3 d c$

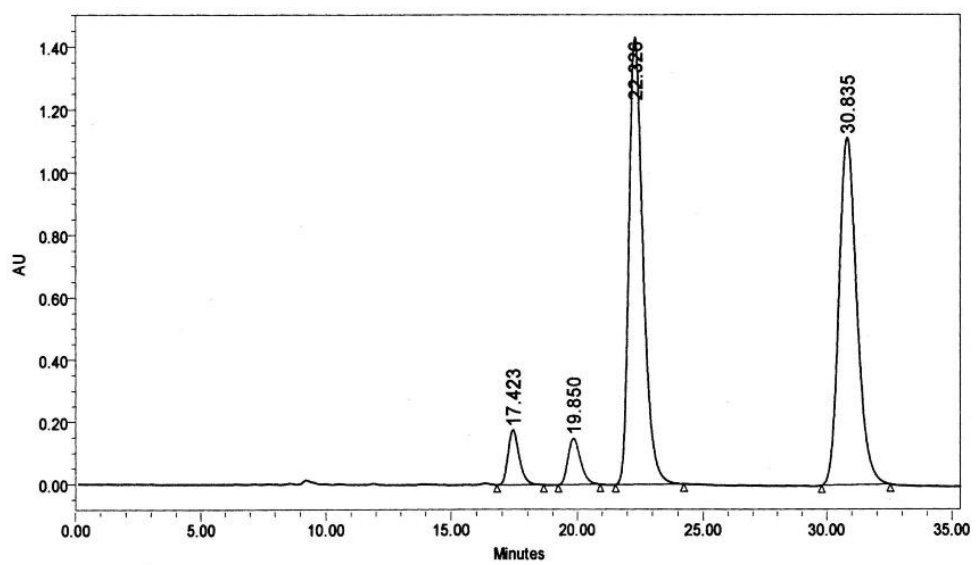

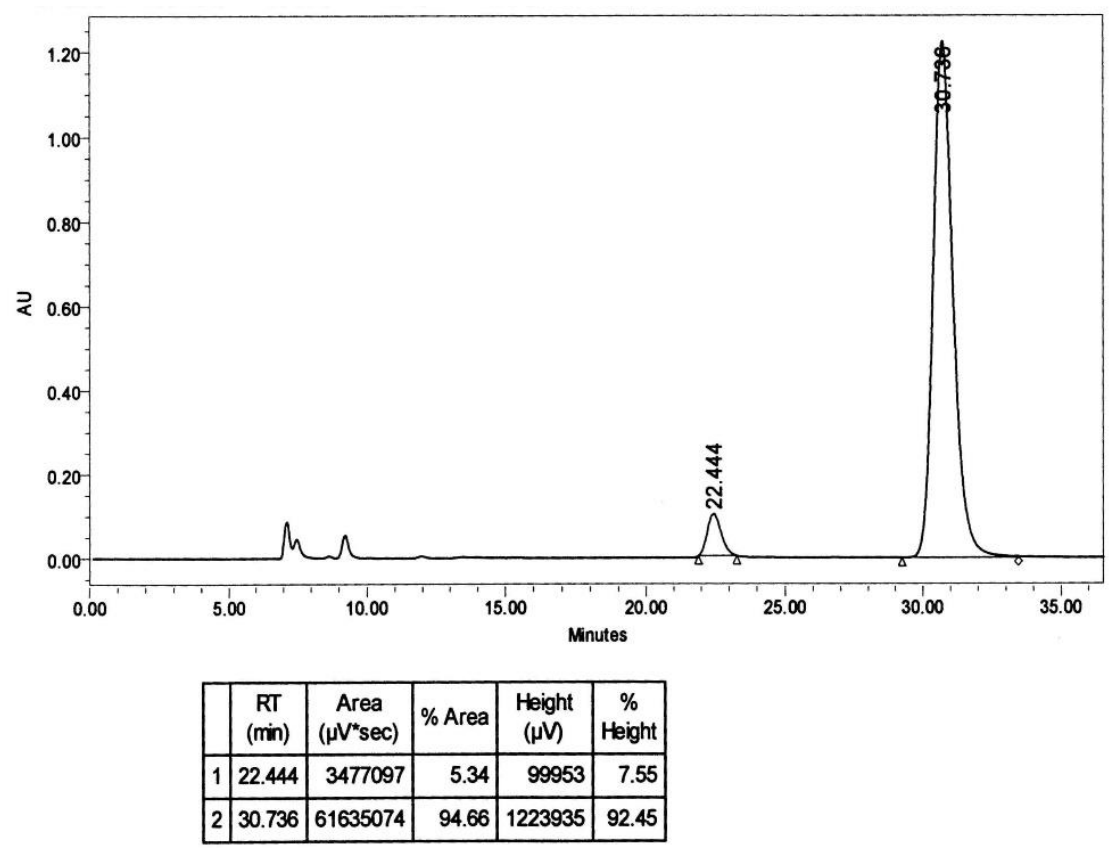



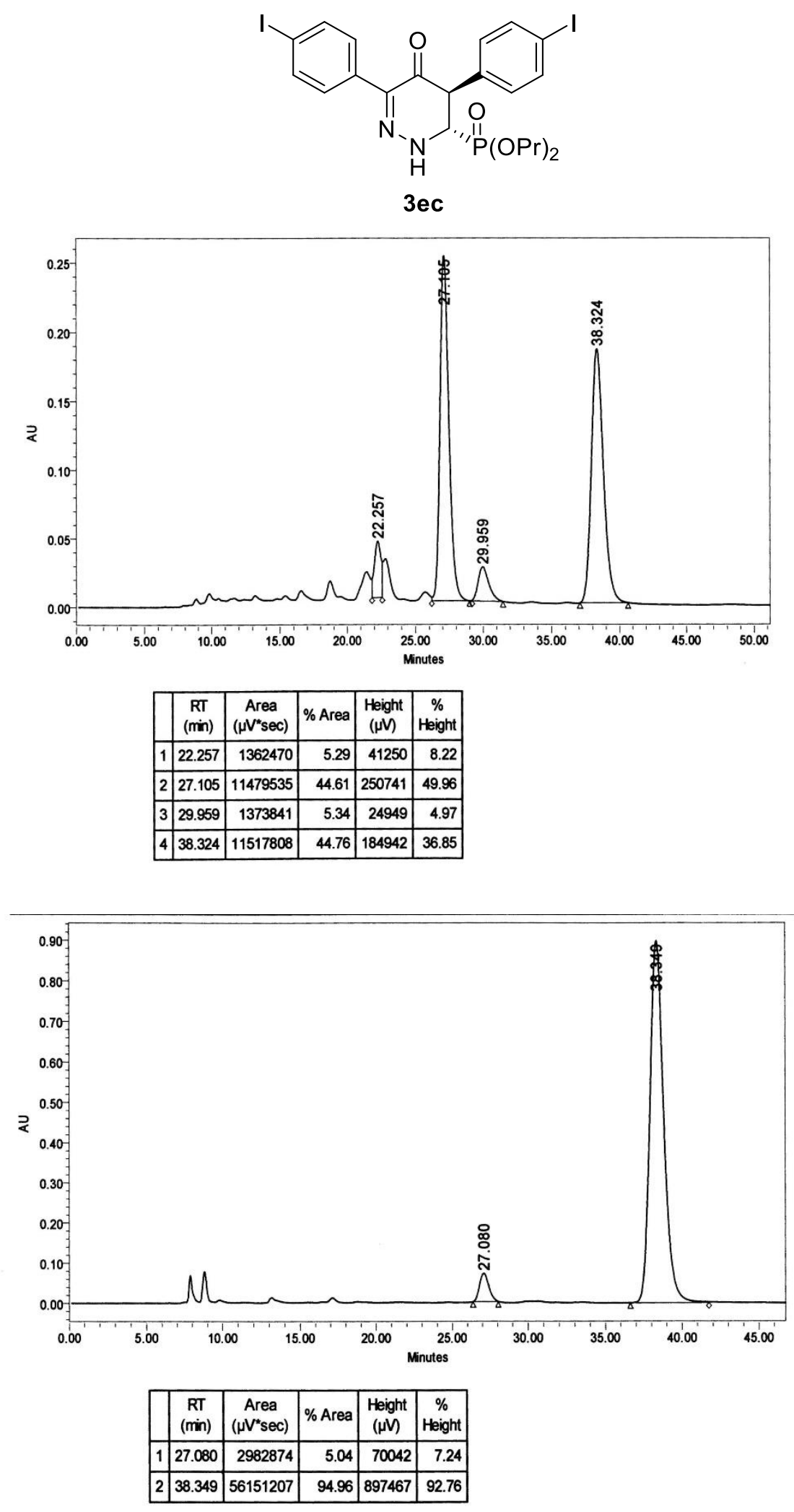


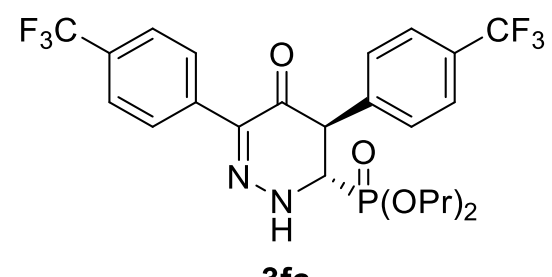

$3 f c$

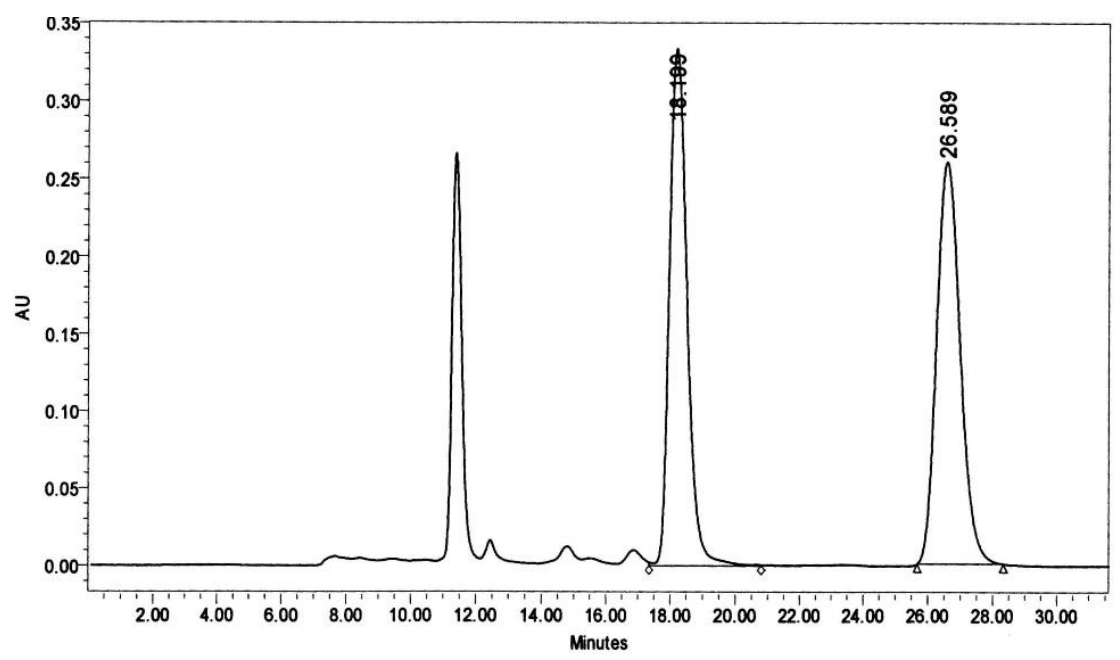

\begin{tabular}{|c|c|c|c|c|c|}
\hline & $\begin{array}{c}\mathrm{RT} \\
(\mathrm{min})\end{array}$ & $\begin{array}{c}\text { Area } \\
(\mu \mathrm{V} * \mathrm{sec})\end{array}$ & $\%$ Area & $\begin{array}{c}\text { Height } \\
(\mu \mathrm{V})\end{array}$ & $\begin{array}{c}\% \\
\text { Height }\end{array}$ \\
\hline 1 & 18.199 & 12736104 & 49.27 & 333961 & 56.24 \\
\hline 2 & 26.589 & 13115920 & 50.73 & 259881 & 43.76 \\
\hline
\end{tabular}

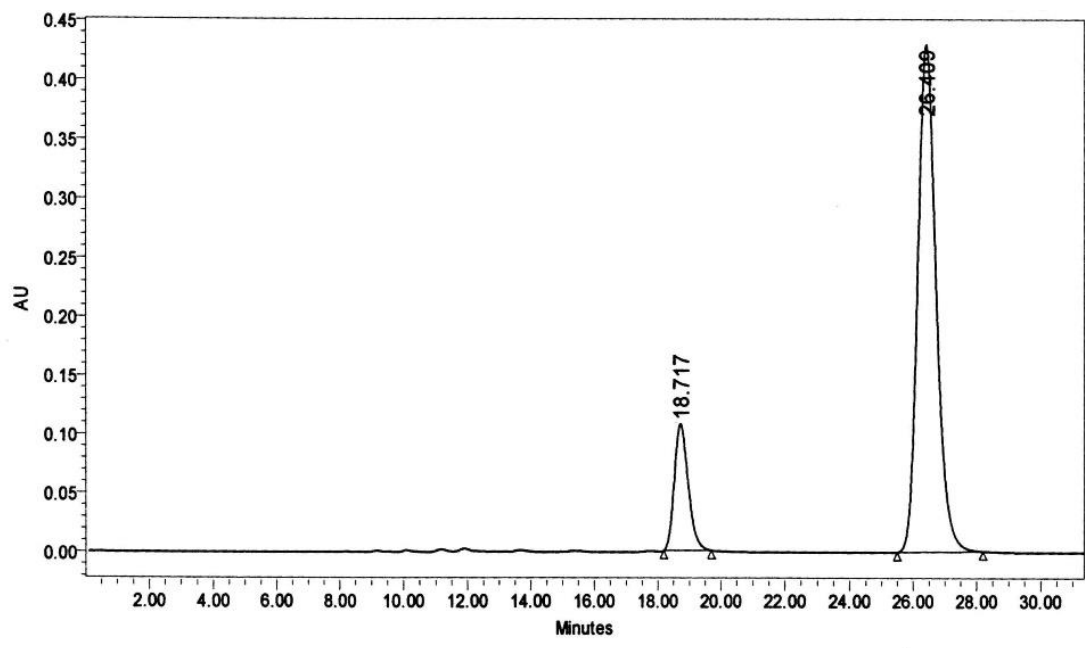

\begin{tabular}{|c|c|c|c|c|c|}
\hline & $\begin{array}{c}\mathrm{RT} \\
(\mathrm{min})\end{array}$ & $\begin{array}{c}\text { Area } \\
(\mu \mathrm{V} * \mathrm{sec})\end{array}$ & $\%$ Area & $\begin{array}{c}\text { Height } \\
(\mu \mathrm{V})\end{array}$ & $\begin{array}{c}\% \\
\text { Height }\end{array}$ \\
\hline 1 & 18.717 & 3299298 & 15.93 & 107719 & 20.04 \\
\hline 2 & 26.409 & 17416918 & 84.07 & 429802 & 79.96 \\
\hline
\end{tabular}



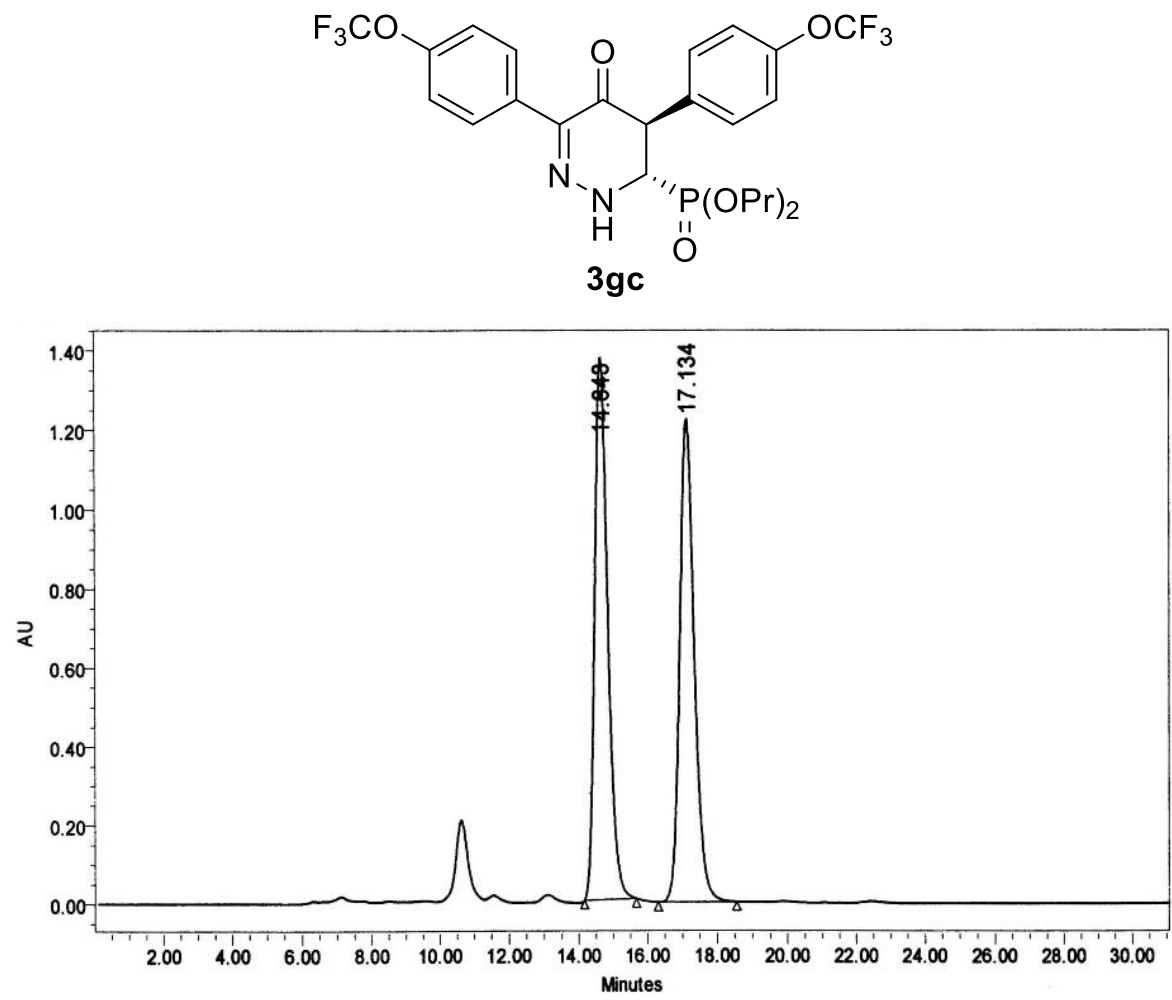

\begin{tabular}{|c|c|c|c|c|c|}
\hline & $\begin{array}{c}\text { RT } \\
(\min )\end{array}$ & $\begin{array}{c}\text { Area } \\
\left(\mu \mathrm{V}^{*} \mathrm{sec}\right)\end{array}$ & $\%$ Area & $\begin{array}{c}\text { Height } \\
(\mu \mathrm{V})\end{array}$ & $\begin{array}{c}\% \\
\text { Height }\end{array}$ \\
\hline 1 & 14.643 & 36351873 & 50.71 & 1372988 & 52.85 \\
\hline 2 & 17.134 & 35338006 & 49.29 & 1224703 & 47.15 \\
\hline
\end{tabular}

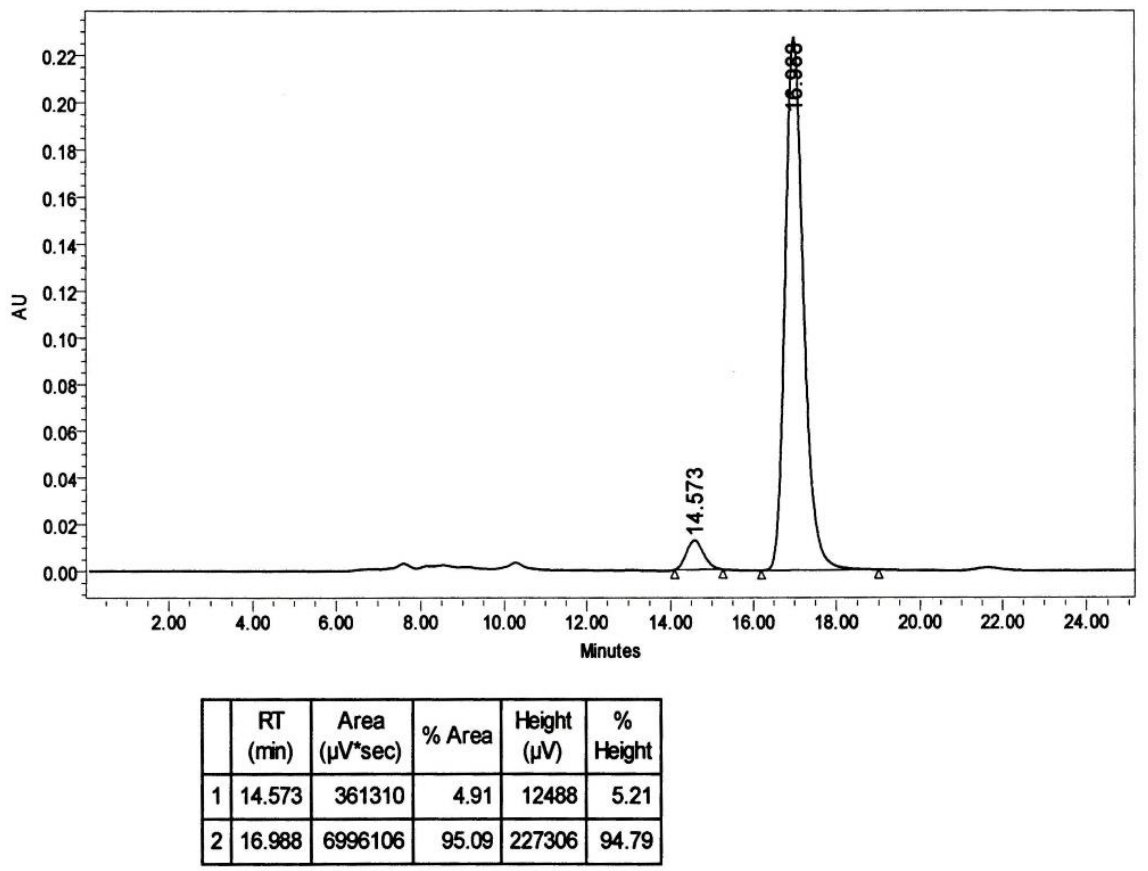


<smiles>O=C1C(c2cccc(F)c2)=NN[C@H]([Pb](=O)O[18OH])[C@H]1c1cccc(F)c1</smiles>

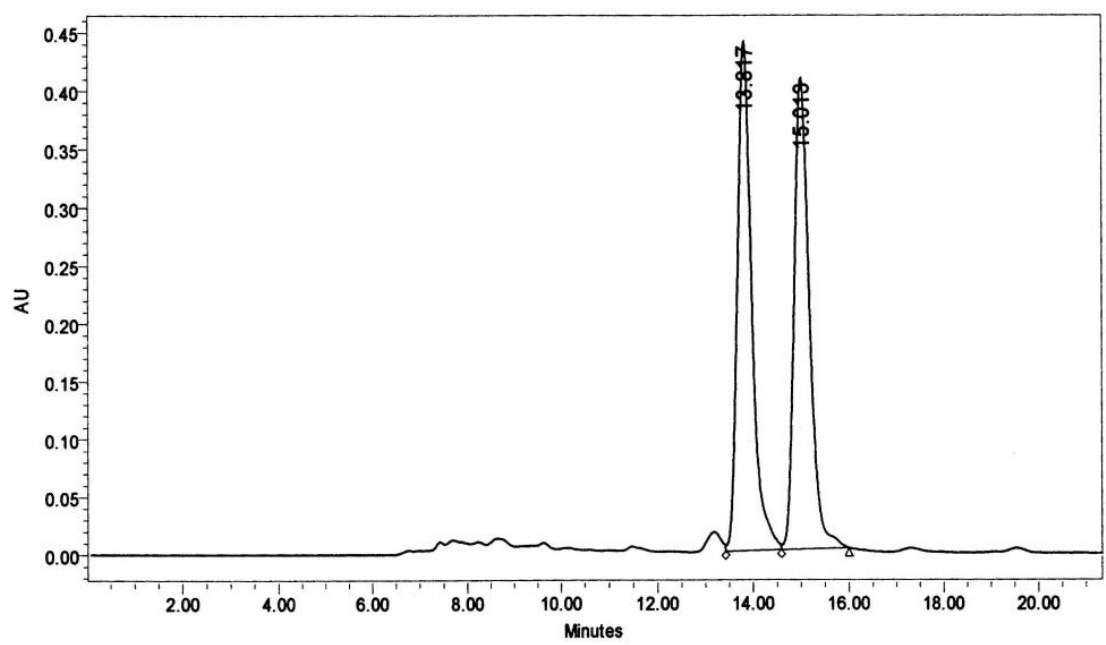

\begin{tabular}{|c|c|c|c|c|c|}
\hline & $\begin{array}{c}\mathrm{RT} \\
(\mathrm{min})\end{array}$ & $\begin{array}{c}\text { Area } \\
(\mu \mathrm{V} * \mathrm{sec})\end{array}$ & $\%$ Area & $\begin{array}{c}\text { Height } \\
(\mu \mathrm{V})\end{array}$ & $\begin{array}{c}\% \\
\text { Height }\end{array}$ \\
\hline 1 & 13.817 & 9199849 & 50.37 & 439628 & 51.93 \\
\hline 2 & 15.013 & 9065615 & 49.63 & 406977 & 48.07 \\
\hline
\end{tabular}

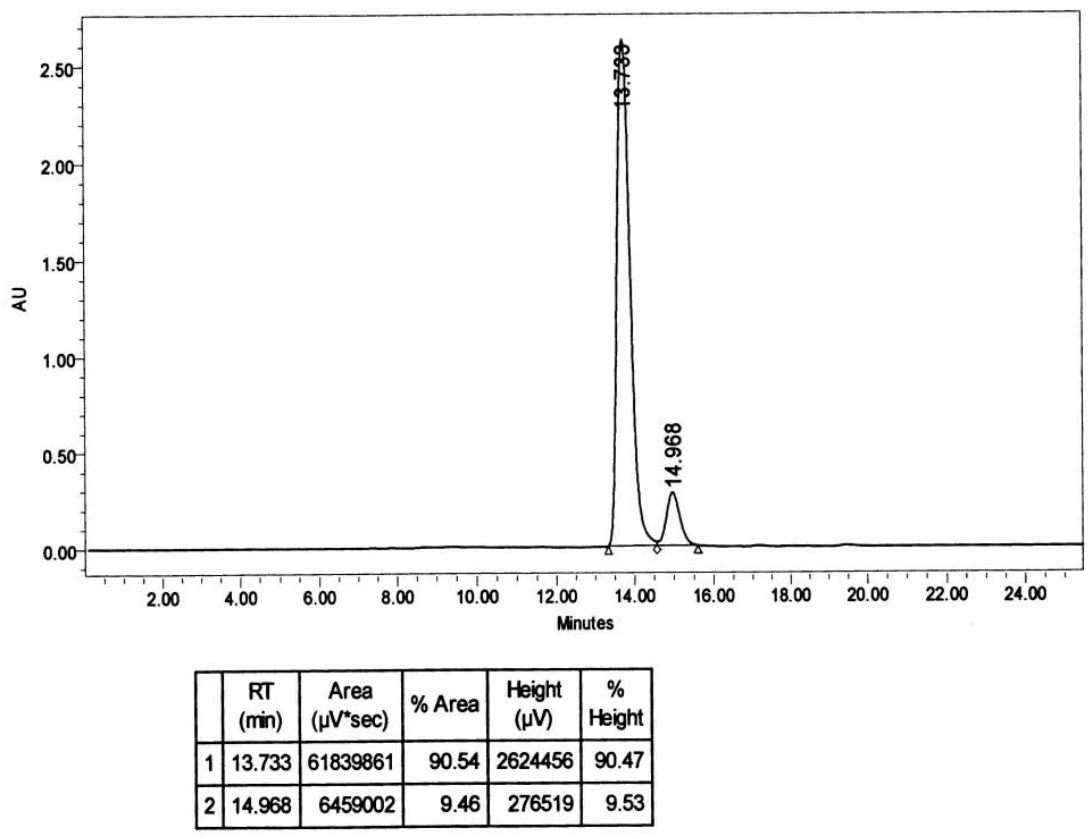




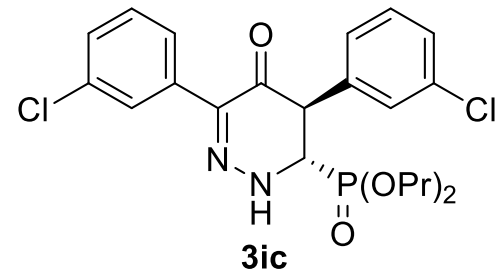

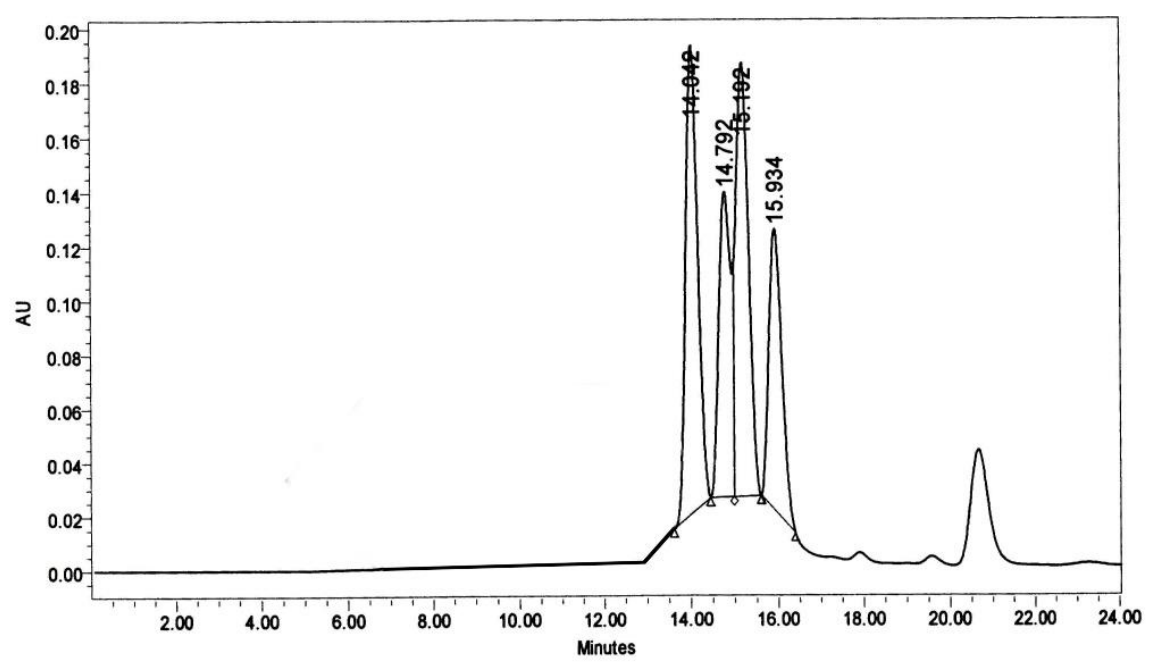

\begin{tabular}{|c|c|c|c|c|c|}
\hline & $\begin{array}{c}\mathrm{RT} \\
(\mathrm{min})\end{array}$ & $\begin{array}{c}\text { Area } \\
(\mu \mathrm{V} \text { sec })\end{array}$ & $\%$ Area & $\begin{array}{c}\text { Height } \\
(\mu \mathrm{V})\end{array}$ & $\begin{array}{c}\% \\
\text { Height }\end{array}$ \\
\hline 1 & 14.042 & 3320847 & 30.35 & 172891 & 31.39 \\
\hline 2 & 14.792 & 2144965 & 19.61 & 113156 & 20.54 \\
\hline 3 & 15.192 & 3284507 & 30.02 & 160310 & 29.10 \\
\hline 4 & 15.934 & 2189849 & 20.02 & 104460 & 18.96 \\
\hline
\end{tabular}

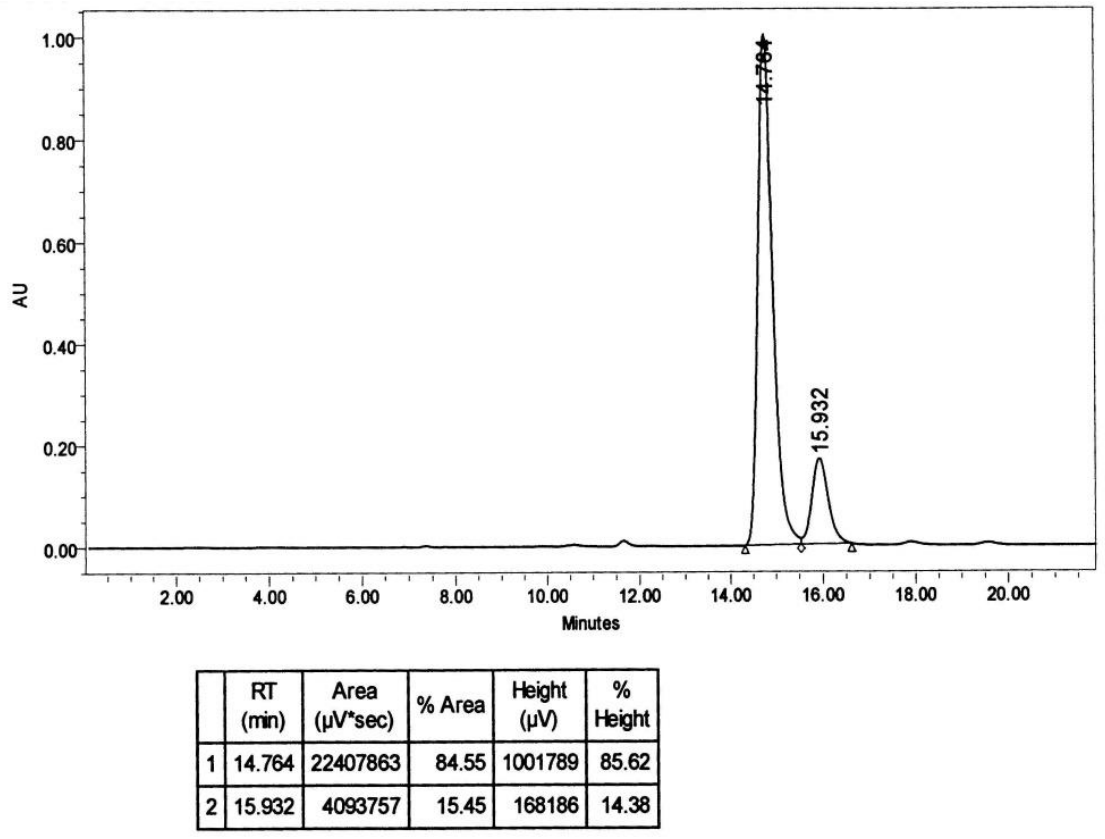



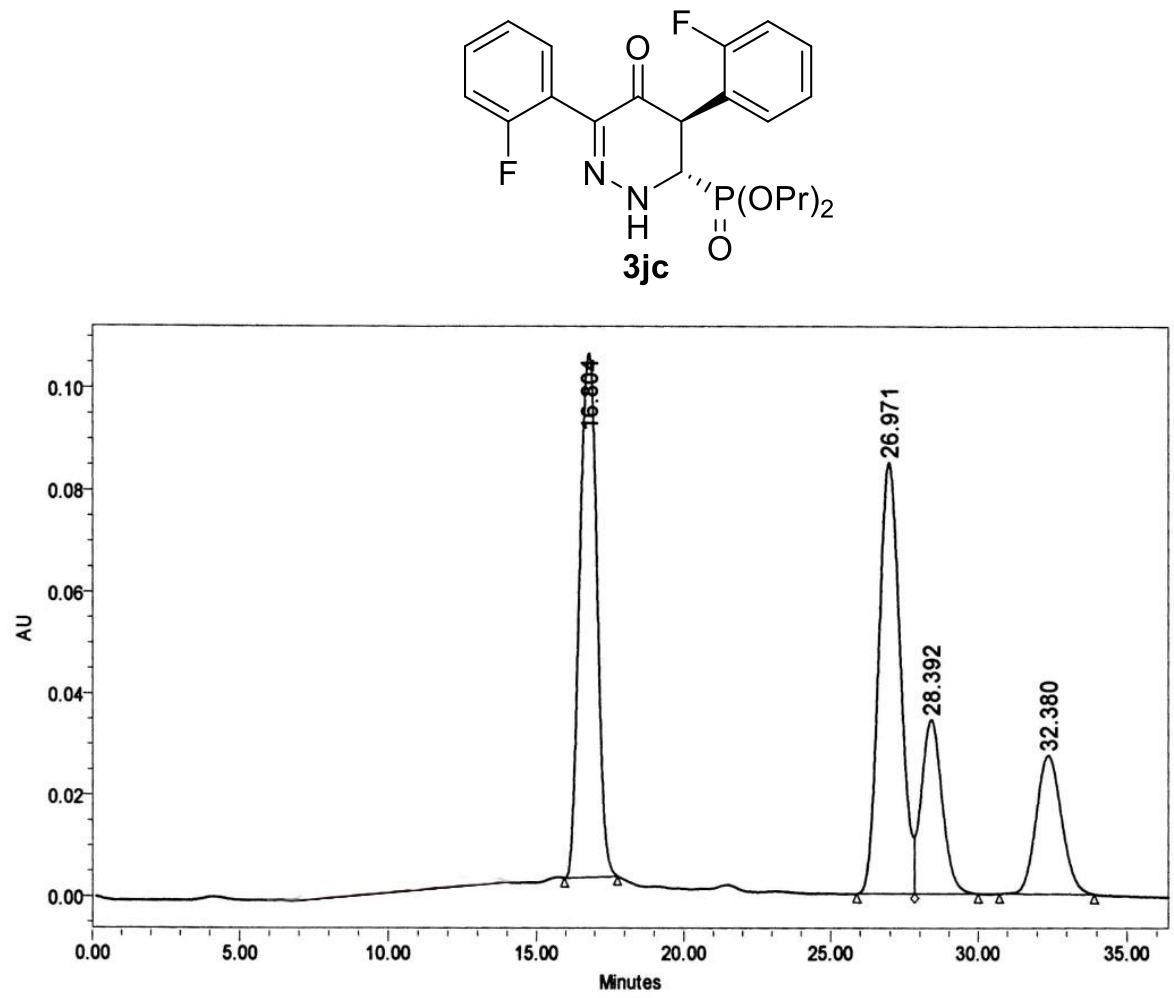

\begin{tabular}{|c|c|c|c|c|c|}
\hline & $\begin{array}{c}\mathrm{RT} \\
(\mathrm{min})\end{array}$ & $\begin{array}{c}\text { Area } \\
\left(\mu \mathrm{V}^{*} \mathrm{sec}\right)\end{array}$ & $\%$ Area & $\begin{array}{c}\text { Height } \\
(\mu \mathrm{V})\end{array}$ & $\begin{array}{c}\% \\
\text { Height }\end{array}$ \\
\hline 1 & 16.804 & 4248175 & 35.92 & 102976 & 41.24 \\
\hline 2 & 26.971 & 4248196 & 35.92 & 84955 & 34.02 \\
\hline 3 & 28.392 & 1710443 & 14.46 & 34372 & 13.77 \\
\hline 4 & 32.380 & 1620383 & 13.70 & 27388 & 10.97 \\
\hline
\end{tabular}

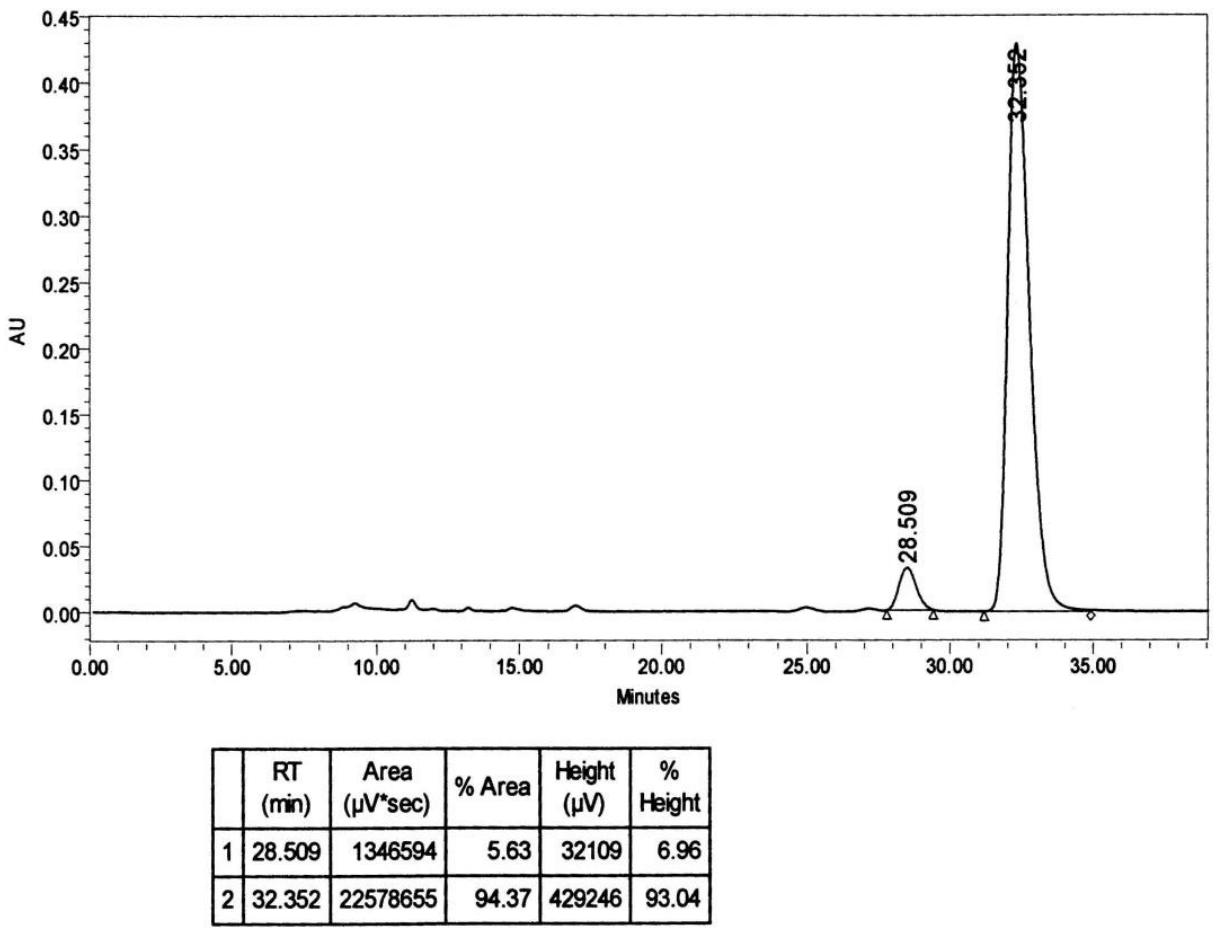


<smiles>CCCCOP(=O)(Oc1ccccc1)[C@H]1NN=C(c2ccccc2Cl)C(=O)[C@@H]1c1ccccc1Cl</smiles>

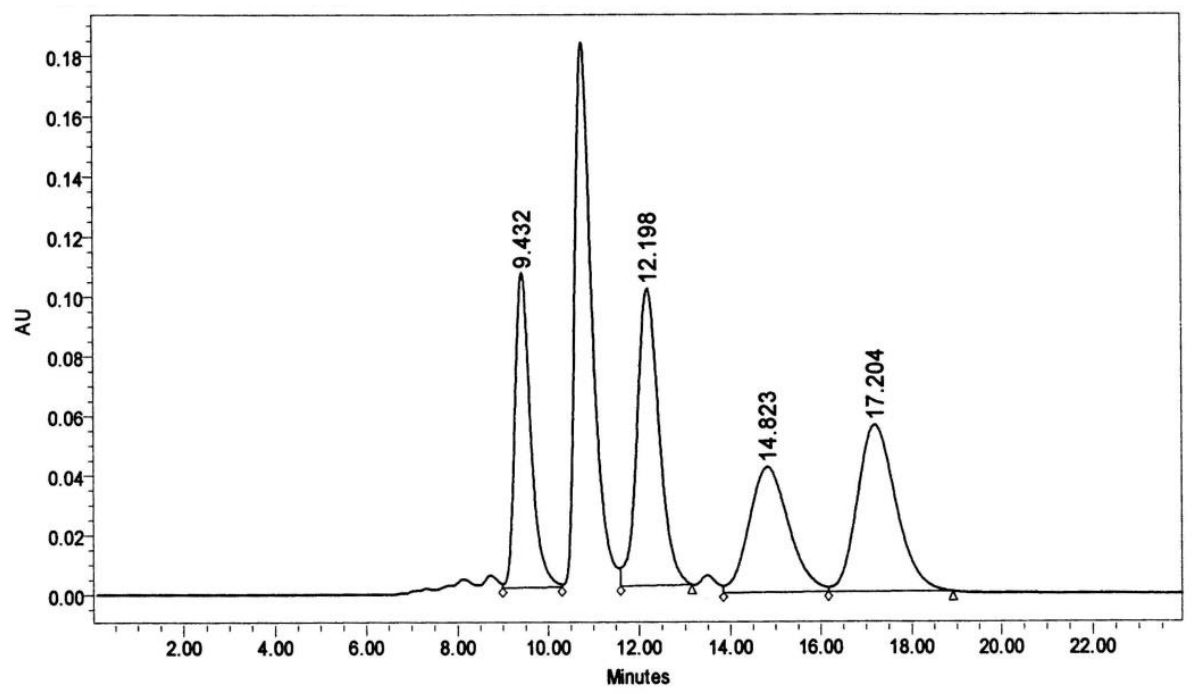

\begin{tabular}{|c|c|c|c|c|c|}
\hline & $\begin{array}{c}\mathrm{RT} \\
(\mathrm{min})\end{array}$ & $\begin{array}{c}\text { Area } \\
(\mu \mathrm{V} * \mathrm{sec})\end{array}$ & $\%$ Area & $\begin{array}{c}\text { Height } \\
(\mu \mathrm{V})\end{array}$ & $\begin{array}{c}\% \\
\text { Height }\end{array}$ \\
\hline 1 & 9.432 & 2556891 & 21.83 & 105617 & 34.84 \\
\hline 2 & 12.198 & 3308568 & 28.25 & 99645 & 32.87 \\
\hline 3 & 14.823 & 2562005 & 21.88 & 42000 & 13.85 \\
\hline 4 & 17.204 & 3283738 & 28.04 & 55929 & 18.45 \\
\hline
\end{tabular}

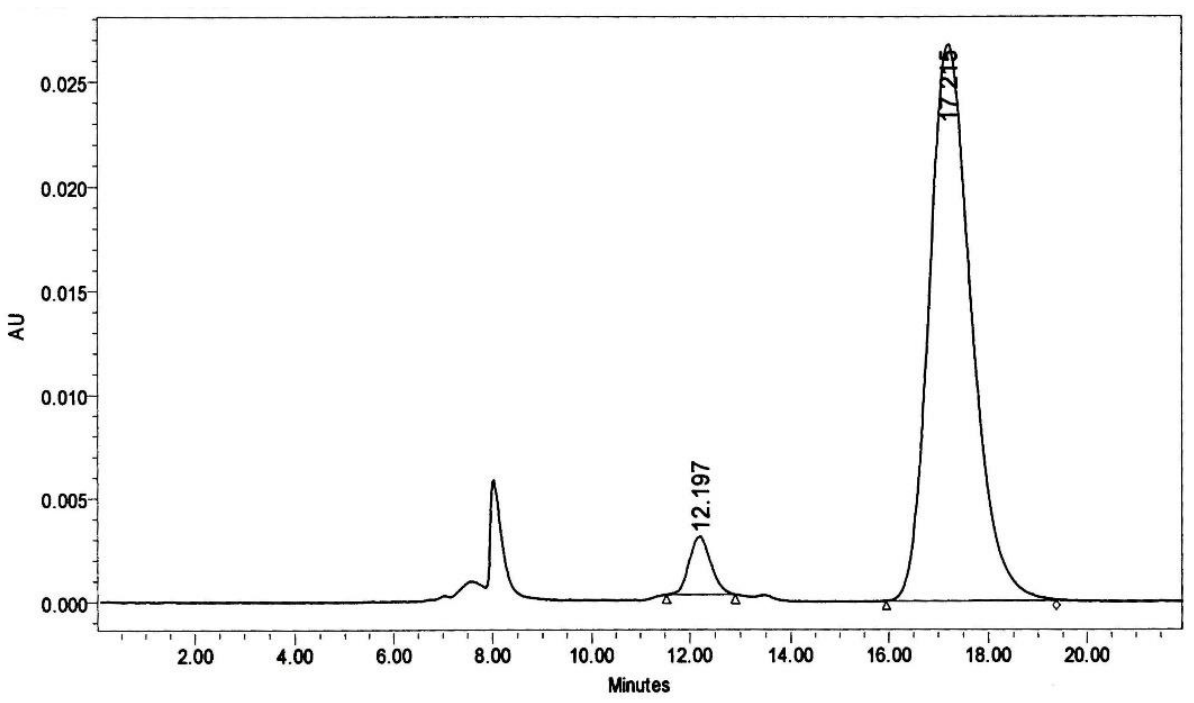

\begin{tabular}{|c|c|r|r|r|r|}
\hline & $\begin{array}{c}\text { RT } \\
(\mathrm{min})\end{array}$ & $\begin{array}{c}\text { Area } \\
\left(\mu \mathrm{V}^{*} \mathrm{sec}\right)\end{array}$ & $\%$ Area & $\begin{array}{c}\text { Height } \\
(\mu \mathrm{V})\end{array}$ & $\begin{array}{c}\% \\
\text { Height }\end{array}$ \\
\hline 1 & 12.197 & 83553 & 5.01 & 2796 & 9.46 \\
\hline 2 & 17.215 & 1583319 & 94.99 & 26750 & 90.54 \\
\hline
\end{tabular}



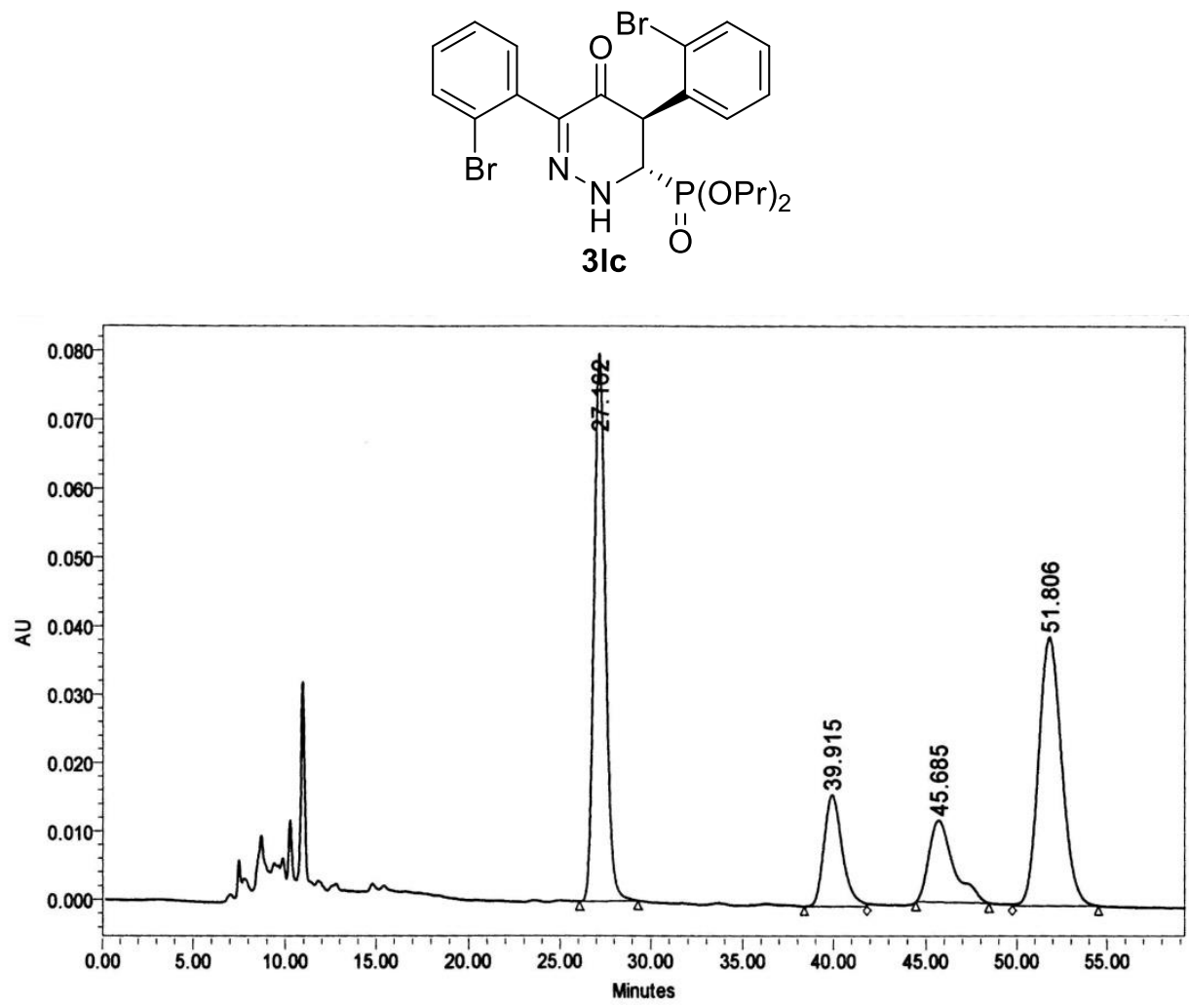

\begin{tabular}{|c|c|c|c|c|c|}
\hline & $\begin{array}{c}\text { RT } \\
(\mathrm{min})\end{array}$ & $\begin{array}{c}\text { Area } \\
\left(\mu \mathrm{V}^{*} \mathrm{sec}\right)\end{array}$ & $\%$ Area & $\begin{array}{c}\text { Height } \\
(\mu \mathrm{V})\end{array}$ & $\begin{array}{c}\% \\
\text { Height }\end{array}$ \\
\hline 1 & 27.162 & 3517495 & 37.92 & 79790 & 54.15 \\
\hline 2 & 39.915 & 1138705 & 12.27 & 16279 & 11.05 \\
\hline 3 & 45.685 & 1117726 & 12.05 & 11952 & 8.11 \\
\hline 4 & 51.806 & 3502990 & 37.76 & 39334 & 26.69 \\
\hline
\end{tabular}

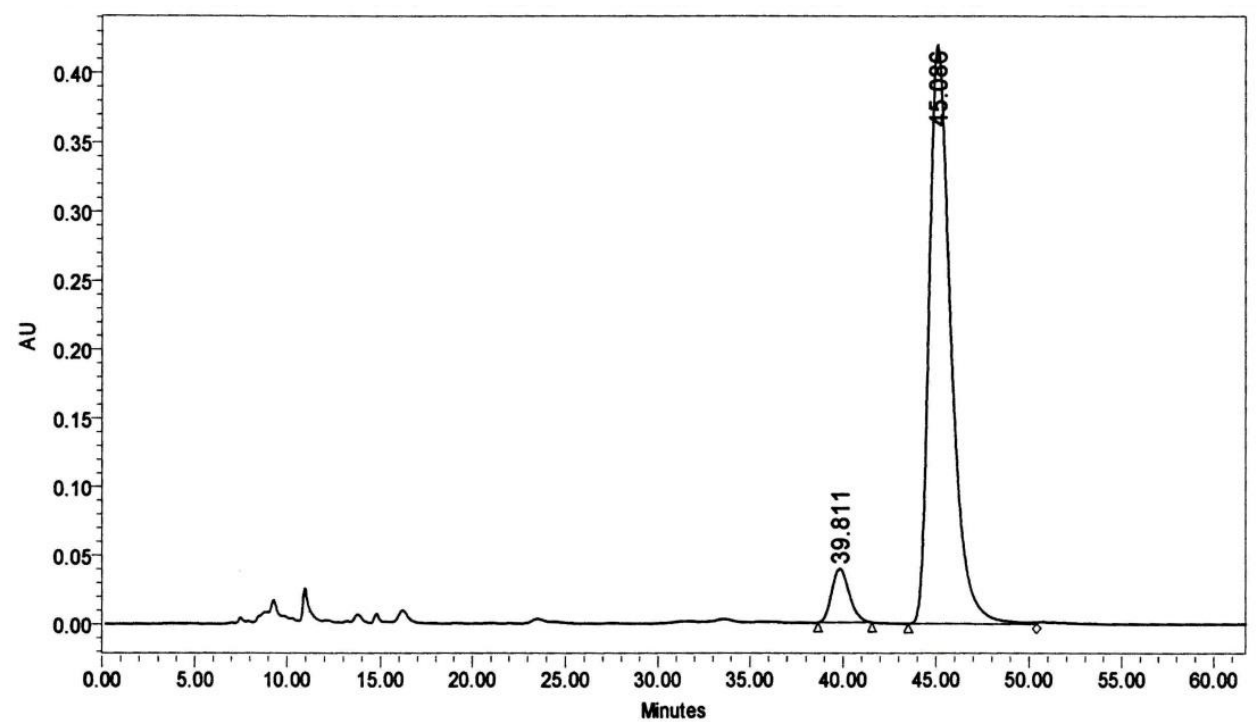

\begin{tabular}{|c|c|c|r|r|r|}
\hline & $\begin{array}{c}\mathrm{RT} \\
(\mathrm{min})\end{array}$ & $\begin{array}{c}\text { Area } \\
(\mu \mathrm{V} * \mathrm{sec})\end{array}$ & $\%$ Area & $\begin{array}{c}\text { Height } \\
(\mu \mathrm{V})\end{array}$ & $\begin{array}{c}\% \\
\text { Height }\end{array}$ \\
\hline 1 & 39.811 & 2572955 & 6.95 & 39156 & 8.53 \\
\hline 2 & 45.086 & 34464108 & 93.05 & 419967 & 91.47 \\
\hline
\end{tabular}



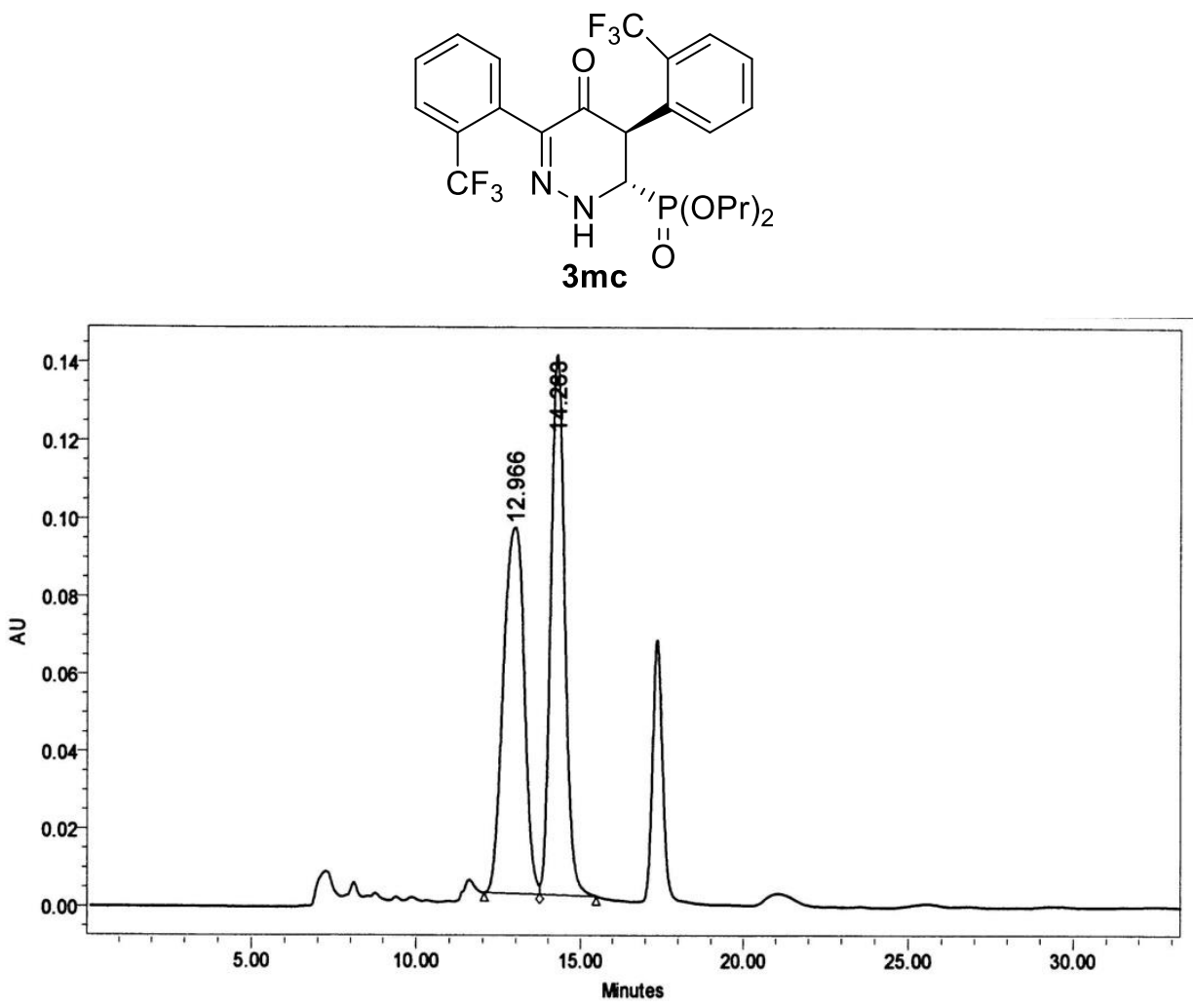

\begin{tabular}{|c|c|c|c|c|c|}
\hline & $\begin{array}{c}\text { RT } \\
(\mathrm{min})\end{array}$ & $\begin{array}{c}\text { Area } \\
(\mu \mathrm{V} \text { *sec })\end{array}$ & $\%$ Area & $\begin{array}{c}\text { Height } \\
(\mu \mathrm{V})\end{array}$ & $\begin{array}{c}\% \\
\text { Height }\end{array}$ \\
\hline 1 & 12.966 & 4201632 & 50.03 & 94480 & 40.47 \\
\hline 2 & 14.263 & 4196744 & 49.97 & 138994 & 59.53 \\
\hline
\end{tabular}

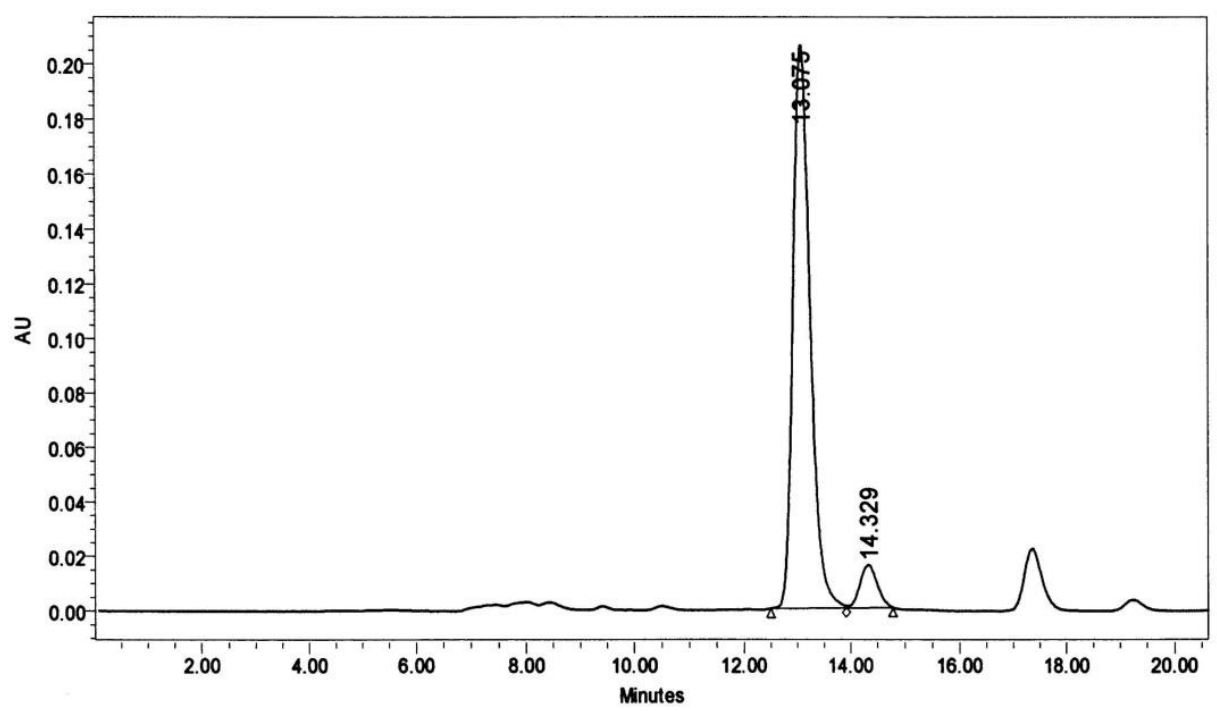

\begin{tabular}{|c|c|c|r|r|r|}
\hline & $\begin{array}{c}\mathrm{RT} \\
(\mathrm{min})\end{array}$ & $\begin{array}{c}\text { Area } \\
\left(\mu \mathrm{V}^{*} \mathrm{sec}\right)\end{array}$ & $\%$ Area & $\begin{array}{c}\text { Height } \\
(\mu \mathrm{V})\end{array}$ & $\begin{array}{c}\% \\
\text { Height }\end{array}$ \\
\hline 1 & 13.075 & 4641285 & 93.01 & 206272 & 92.93 \\
\hline 2 & 14.329 & 348605 & 6.99 & 15692 & 7.07 \\
\hline
\end{tabular}



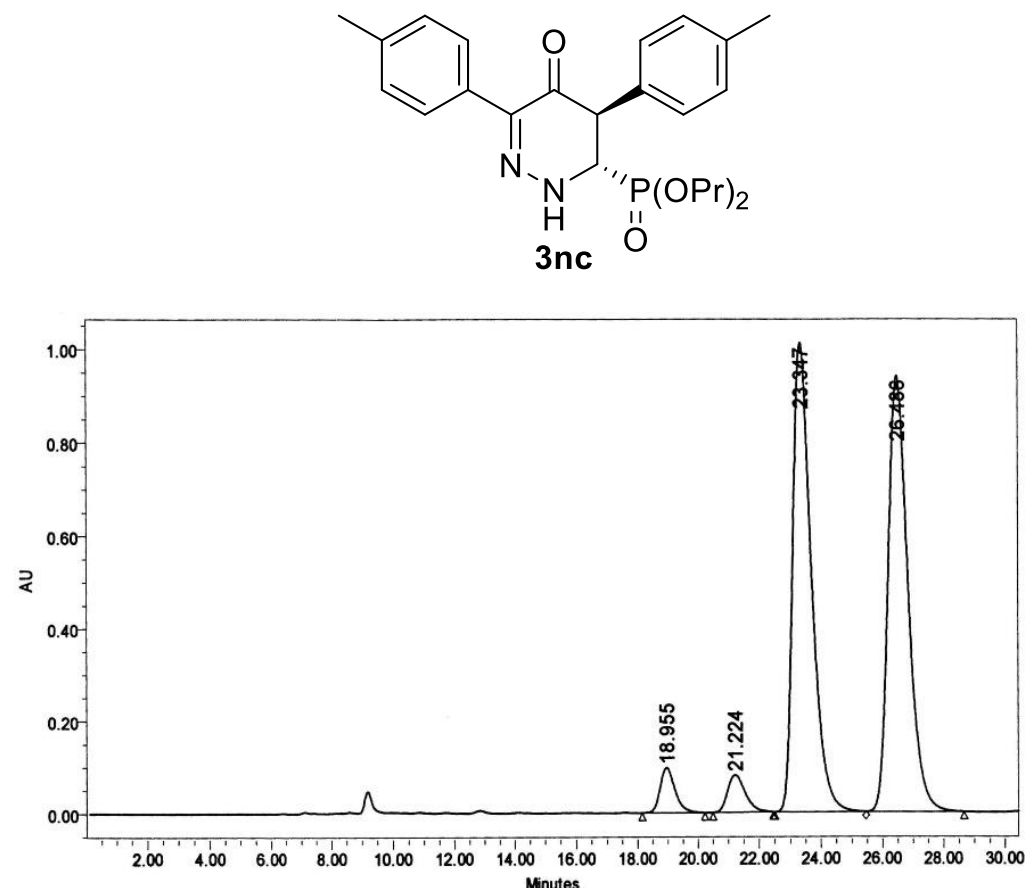

\begin{tabular}{|r|c|c|r|r|r|}
\hline & $\begin{array}{c}\text { RT } \\
(\mathrm{min})\end{array}$ & $\begin{array}{c}\text { Area } \\
\left(\mu \mathrm{V}^{*} \mathrm{sec}\right)\end{array}$ & $\%$ Area & $\begin{array}{c}\text { Height } \\
(\mu \mathrm{V})\end{array}$ & $\begin{array}{c}\% \\
\text { Height }\end{array}$ \\
\hline 1 & 18.955 & 3319640 & 3.65 & 97157 & 4.57 \\
\hline 2 & 21.224 & 3210734 & 3.53 & 80231 & 3.77 \\
\hline 3 & 23.347 & 42149987 & 46.35 & 1010692 & 47.50 \\
\hline 4 & 26.486 & 42264179 & 46.47 & 939571 & 44.16 \\
\hline
\end{tabular}

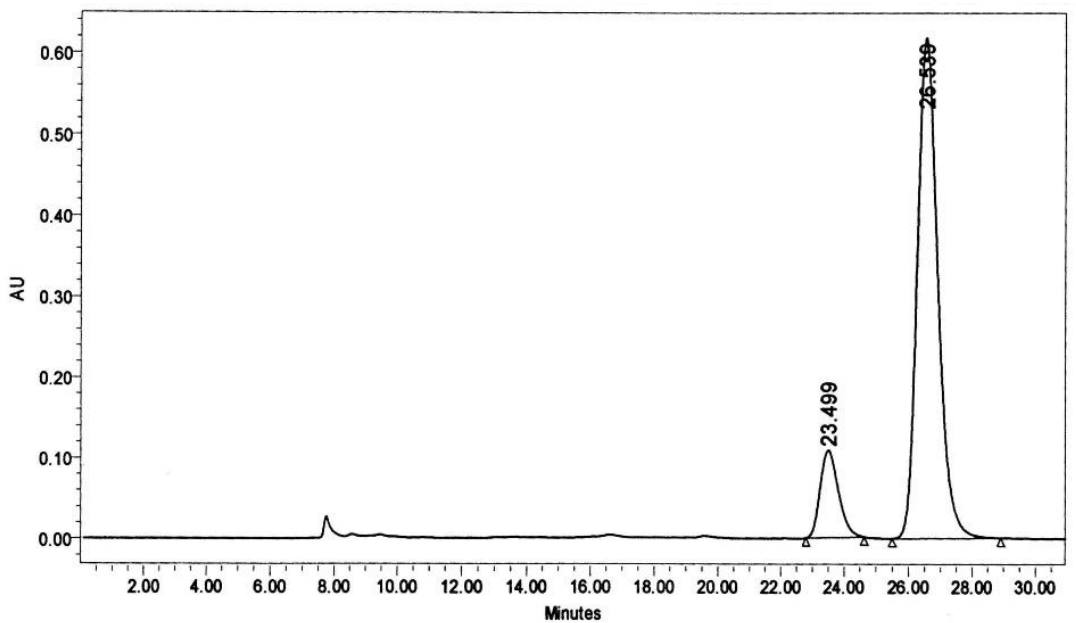

\begin{tabular}{|c|c|c|c|c|c|}
\hline & $\begin{array}{c}\text { RT } \\
(\min )\end{array}$ & $\begin{array}{c}\text { Area } \\
\left(\mu \mathrm{V}^{*} \mathrm{sec}\right)\end{array}$ & $\%$ Area & $\begin{array}{c}\text { Height } \\
(\mu \mathrm{V})\end{array}$ & $\begin{array}{c}\% \\
\text { Height }\end{array}$ \\
\hline 1 & 23.499 & 4330576 & 13.53 & 108417 & 14.93 \\
\hline 2 & 26.539 & 27684679 & 86.47 & 617752 & 85.07 \\
\hline
\end{tabular}



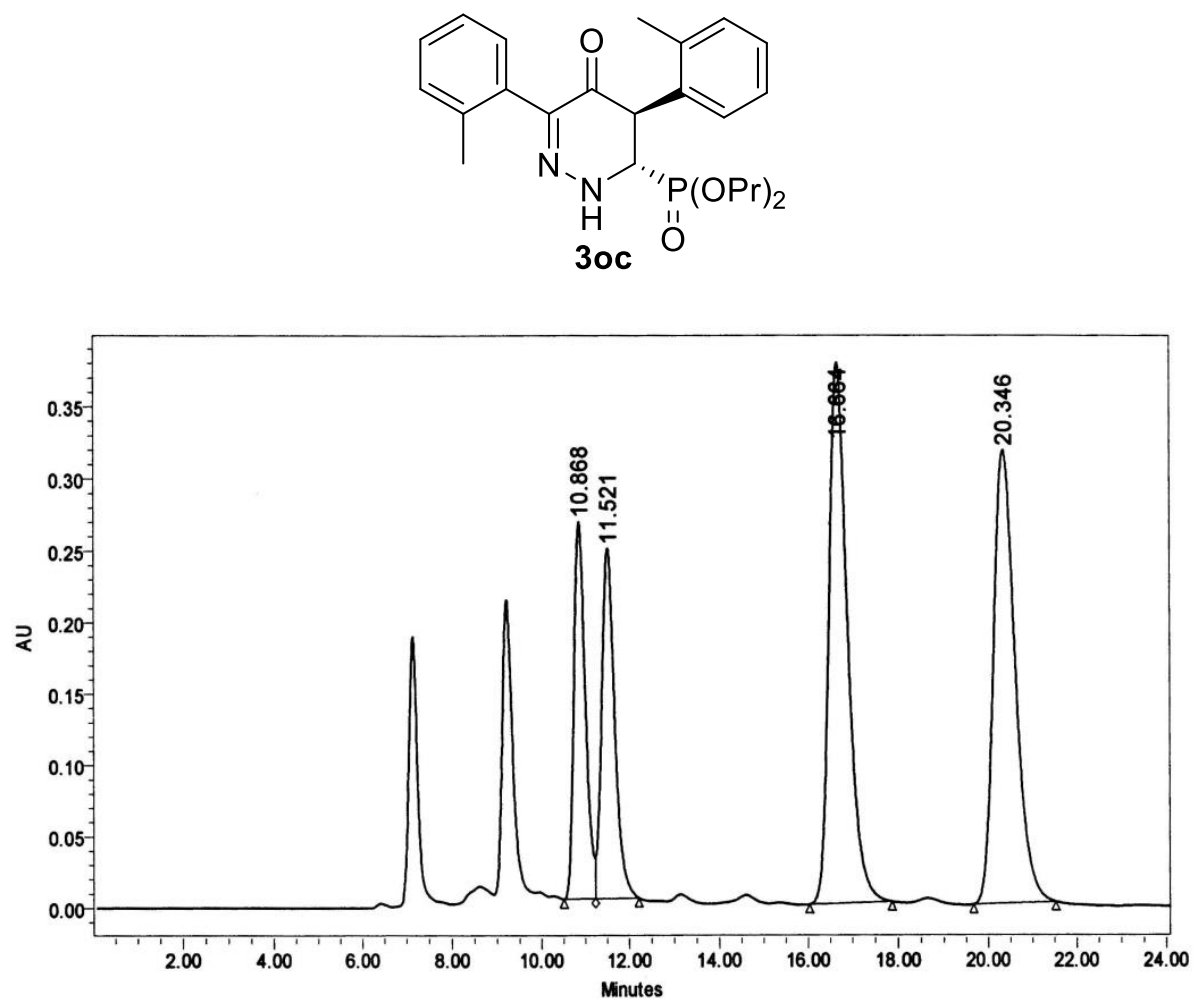

\begin{tabular}{|c|c|c|c|c|c|}
\hline & $\begin{array}{c}\mathrm{RT} \\
(\mathrm{min})\end{array}$ & $\begin{array}{c}\text { Area } \\
\left(\mu \mathrm{V}^{*} \mathrm{sec}\right)\end{array}$ & $\%$ Area & $\begin{array}{c}\text { Height } \\
(\mu \mathrm{V})\end{array}$ & $\begin{array}{c}\% \\
\text { Height }\end{array}$ \\
\hline 1 & 10.868 & 4817675 & 15.21 & 264115 & 21.94 \\
\hline 2 & 11.521 & 5082525 & 16.04 & 245308 & 20.38 \\
\hline 3 & 16.664 & 10891199 & 34.38 & 377579 & 31.36 \\
\hline 4 & 20.346 & 10890465 & 34.37 & 316910 & 26.32 \\
\hline
\end{tabular}

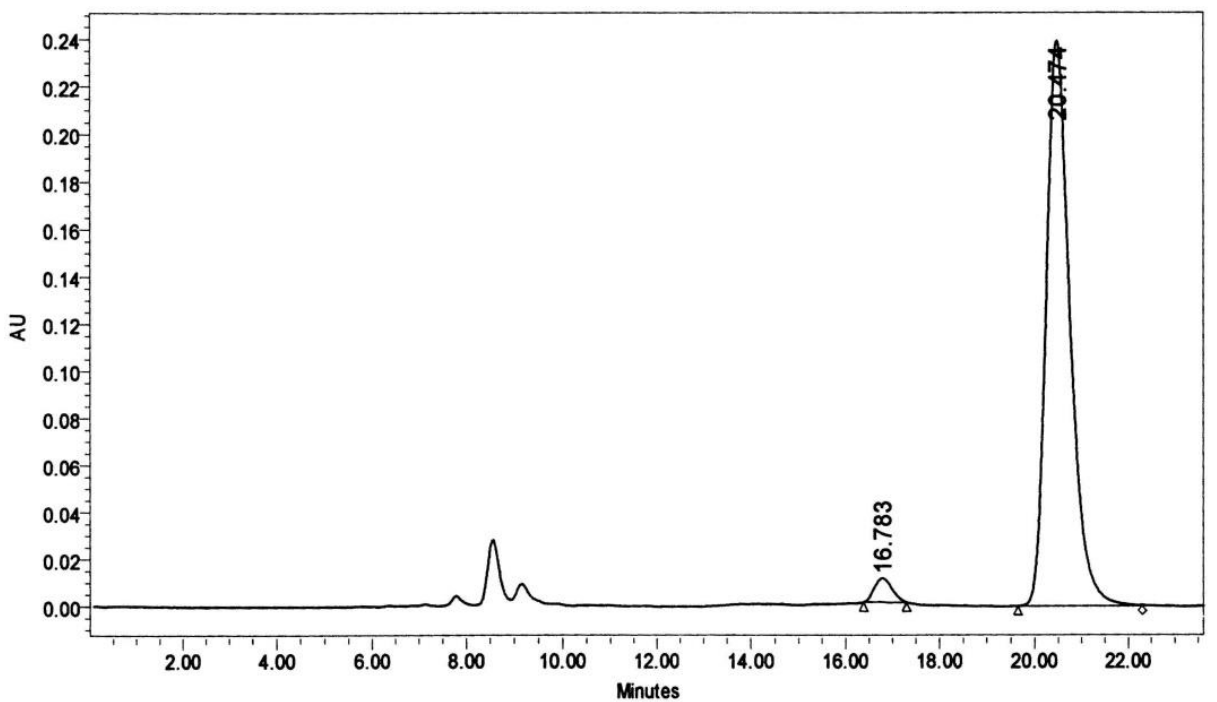

\begin{tabular}{|c|c|c|c|c|c|}
\hline & $\begin{array}{c}\mathrm{RT} \\
(\mathrm{min})\end{array}$ & $\begin{array}{c}\text { Area } \\
\left(\mu \mathrm{V}^{*} \mathrm{sec}\right)\end{array}$ & $\%$ Area & $\begin{array}{c}\text { Height } \\
(\mu \mathrm{V})\end{array}$ & $\begin{array}{c}\% \\
\text { Height }\end{array}$ \\
\hline 1 & 16.783 & 265823 & 3.05 & 10167 & 4.08 \\
\hline 2 & 20.474 & 8441725 & 96.95 & 239253 & 95.92 \\
\hline
\end{tabular}


<smiles>O=C(OP)[C@H]1NN=C(c2ccc(F)cc2F)C(=O)[C@@H]1c1ccc(F)cc1F</smiles>

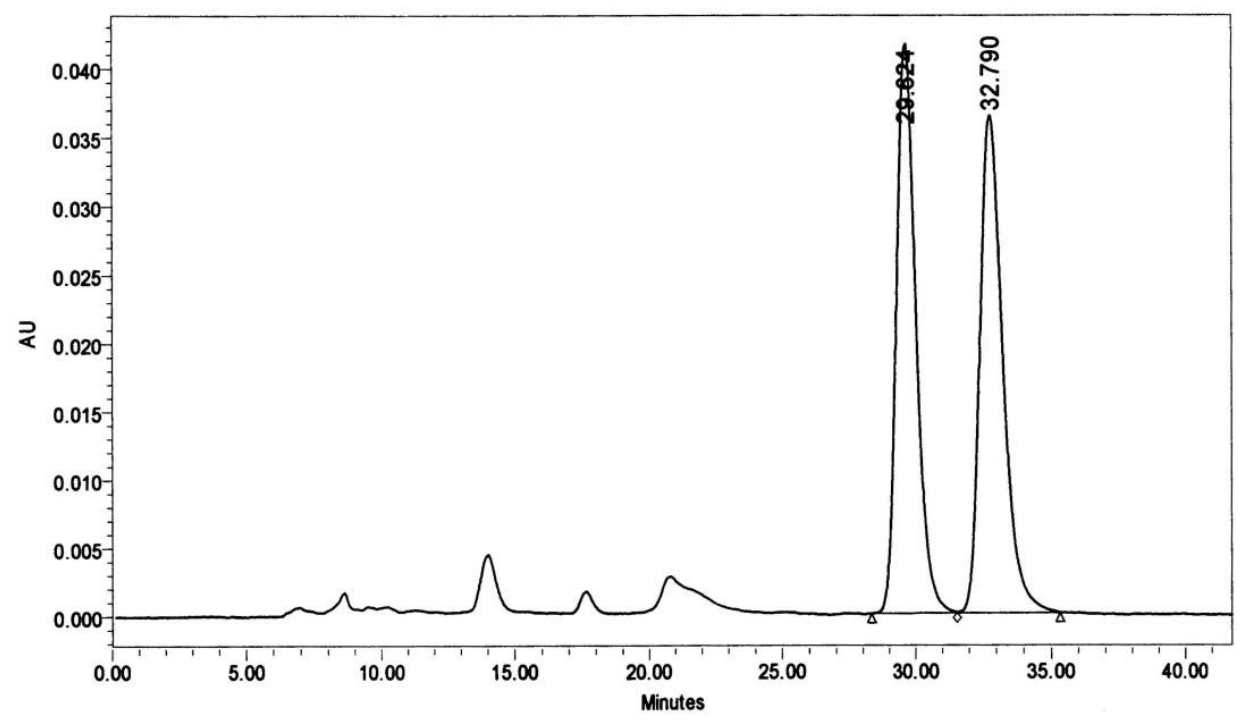

\begin{tabular}{|c|c|c|c|c|c|}
\hline & $\begin{array}{c}\mathrm{RT} \\
(\mathrm{min})\end{array}$ & $\begin{array}{c}\text { Area } \\
(\mu \mathrm{N} * \mathrm{sec})\end{array}$ & $\%$ Area & $\begin{array}{c}\text { Height } \\
(\mu \mathrm{V})\end{array}$ & $\begin{array}{c}\% \\
\text { Height }\end{array}$ \\
\hline 1 & 29.624 & 2151566 & 50.15 & 41603 & 53.35 \\
\hline 2 & 32.790 & 2138966 & 49.85 & 36381 & 46.65 \\
\hline
\end{tabular}

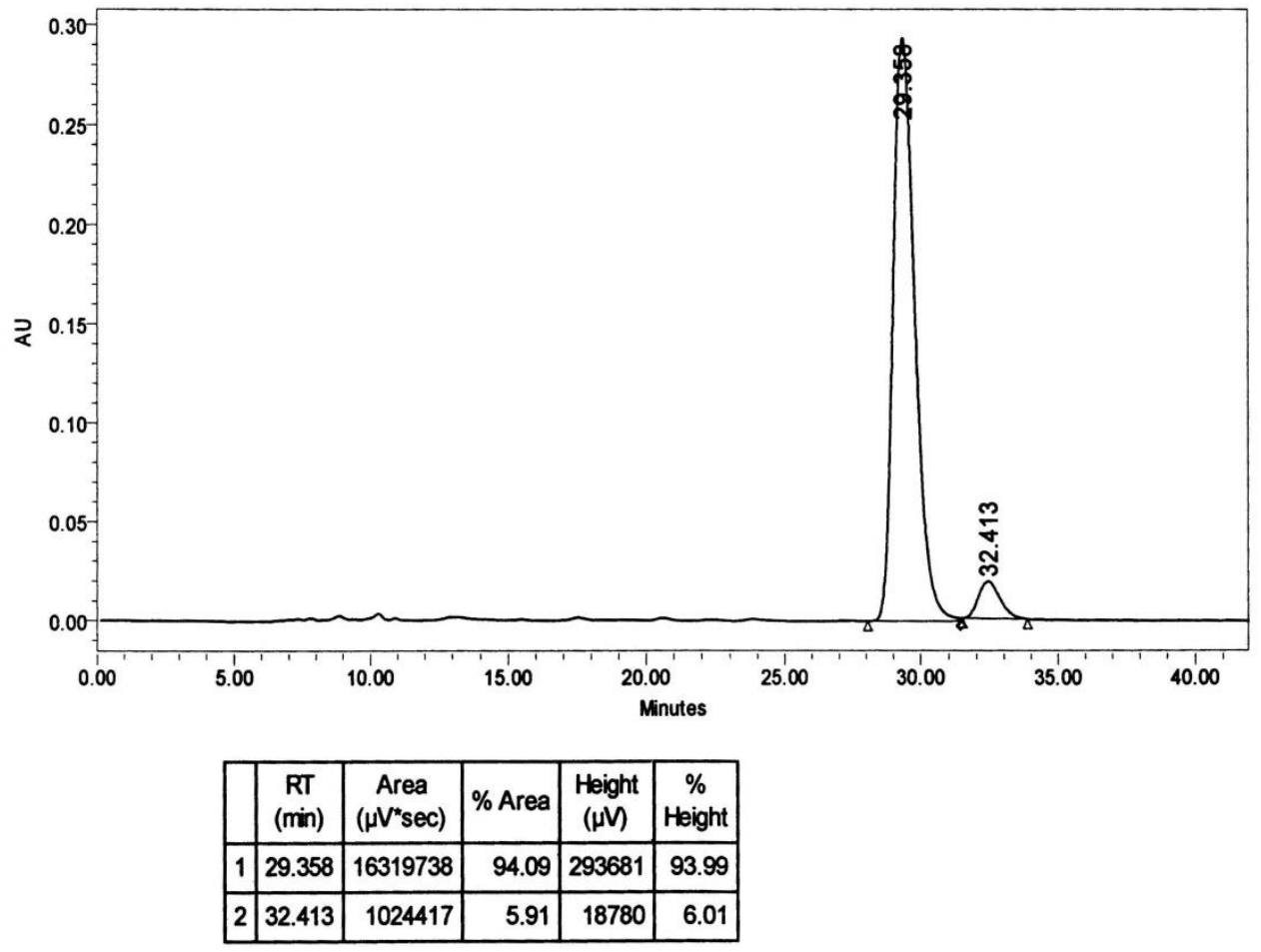



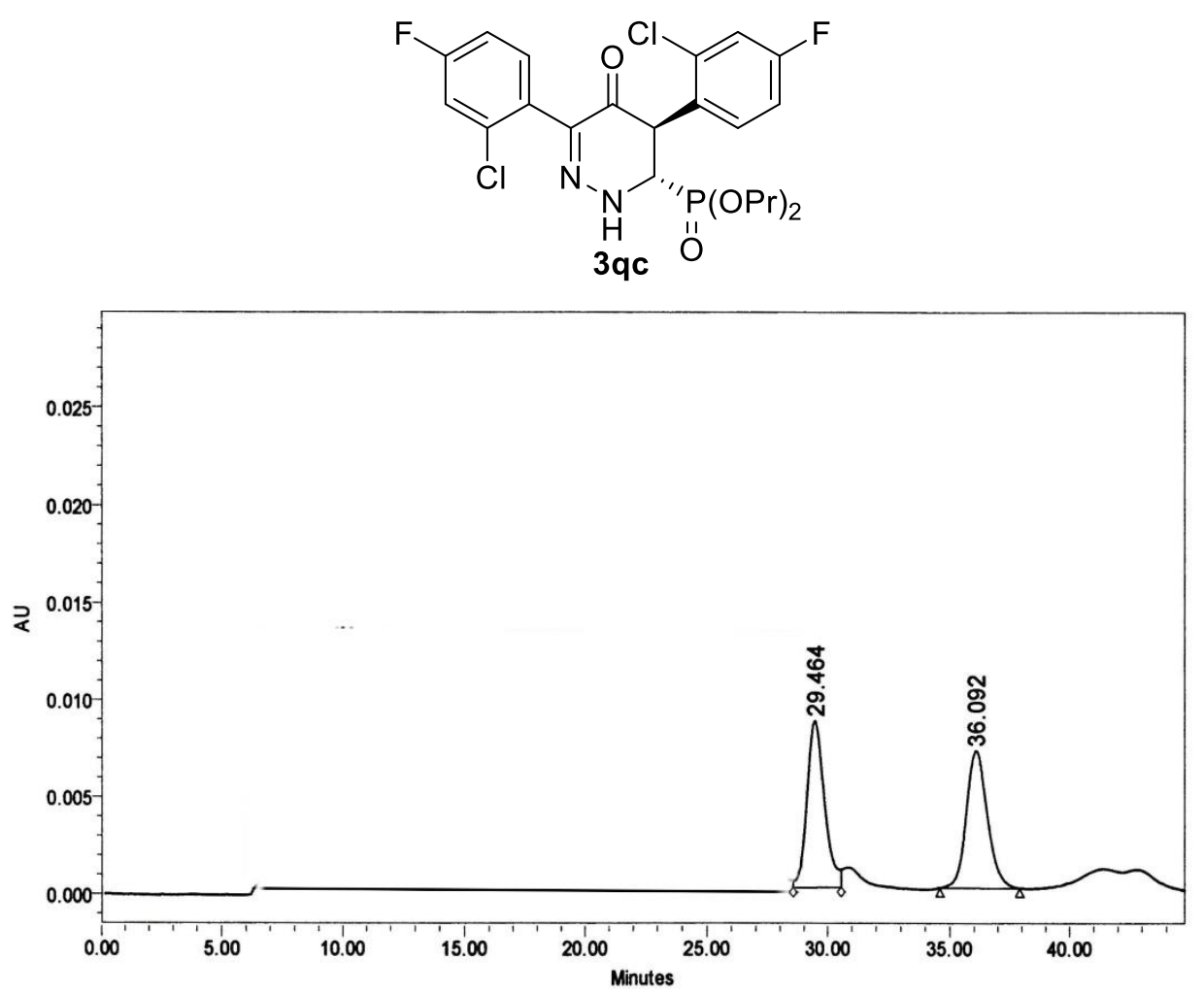

\begin{tabular}{|c|c|c|c|c|c|}
\hline & $\begin{array}{c}\mathrm{RT} \\
(\mathrm{min})\end{array}$ & $\begin{array}{c}\text { Area } \\
(\mu \mathrm{V} * \mathrm{sec})\end{array}$ & $\%$ Area & $\begin{array}{c}\text { Height } \\
(\mu \mathrm{V})\end{array}$ & $\begin{array}{c}\% \\
\text { Height }\end{array}$ \\
\hline 1 & 29.464 & 441257 & 50.50 & 8593 & 54.82 \\
\hline 2 & 36.092 & 432452 & 49.50 & 7082 & 45.18 \\
\hline
\end{tabular}

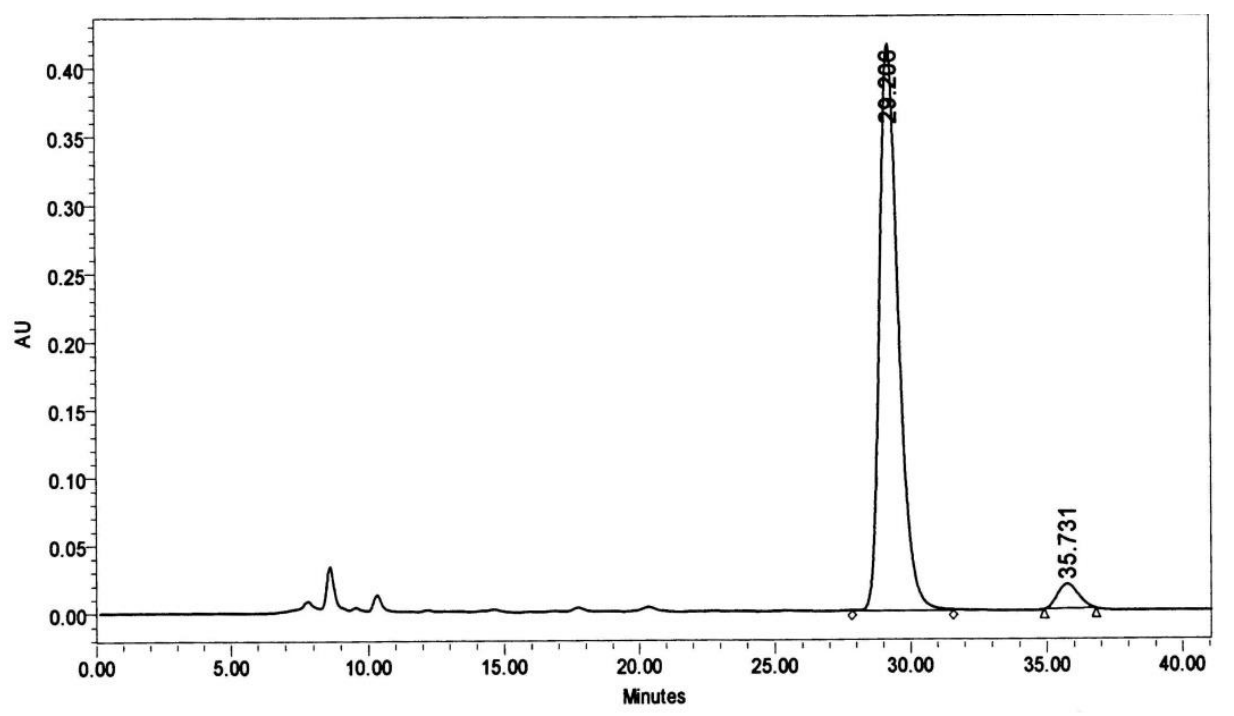

\begin{tabular}{|c|c|r|r|r|r|}
\hline & $\begin{array}{c}\mathrm{RT} \\
(\mathrm{min})\end{array}$ & $\begin{array}{c}\text { Area } \\
(\mu \mathrm{V} \text { sec })\end{array}$ & $\%$ Area & $\begin{array}{c}\text { Height } \\
(\mu \mathrm{V})\end{array}$ & $\begin{array}{c}\% \\
\text { Height }\end{array}$ \\
\hline 1 & 29.206 & 20390748 & 95.49 & 415383 & 95.85 \\
\hline 2 & 35.731 & 962866 & 4.51 & 17992 & 4.15 \\
\hline
\end{tabular}



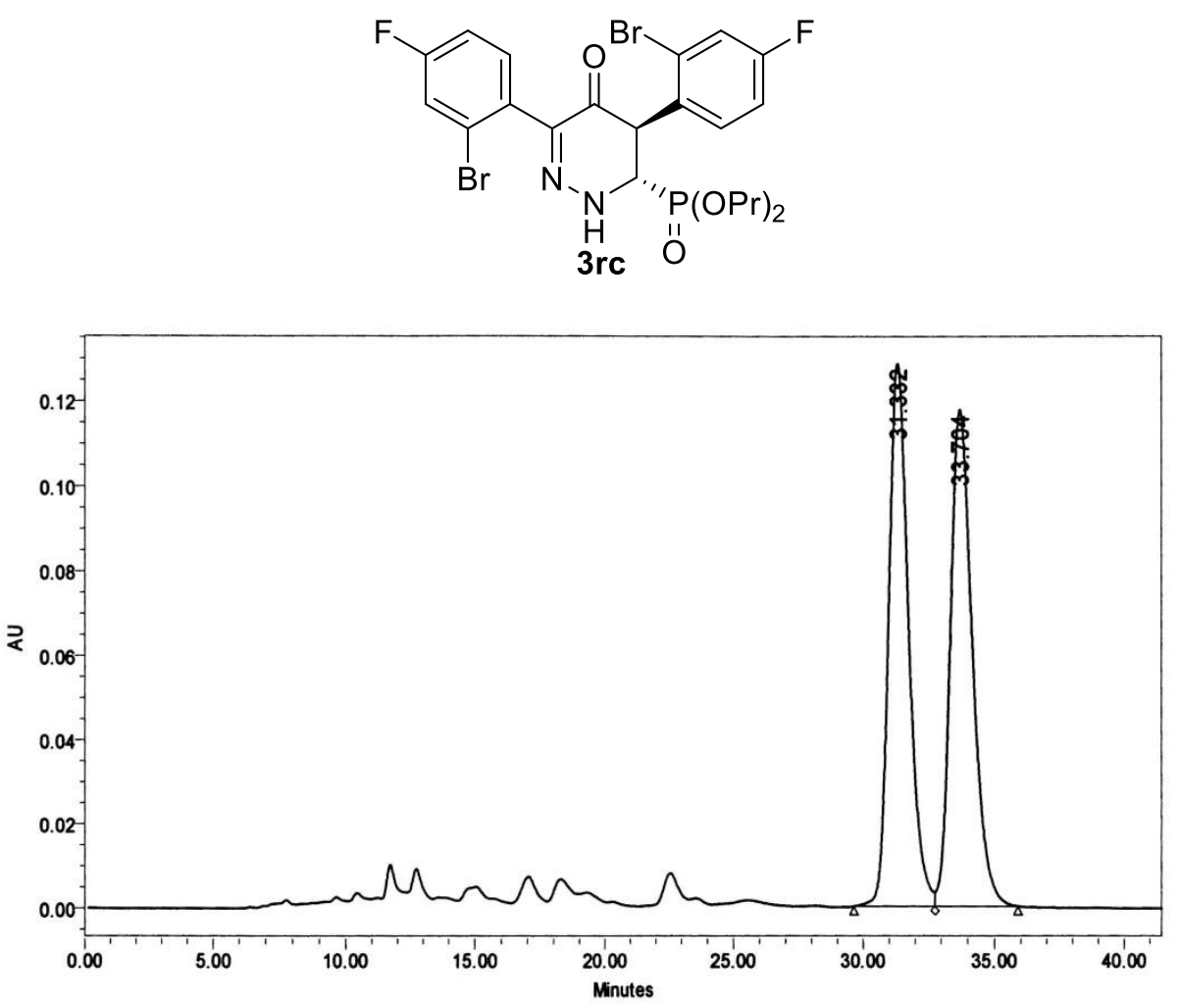

\begin{tabular}{|c|c|c|c|c|c|}
\hline & $\begin{array}{c}\text { RT } \\
(\mathrm{min})\end{array}$ & $\begin{array}{c}\text { Area } \\
\left(\mu \mathrm{V}{ }^{*} \mathrm{sec}\right)\end{array}$ & $\%$ Area & $\begin{array}{c}\text { Height } \\
(\mu \mathrm{V})\end{array}$ & $\begin{array}{c}\% \\
\text { Height }\end{array}$ \\
\hline 1 & 31.332 & 6849471 & 50.17 & 128437 & 52.19 \\
\hline 2 & 33.704 & 6802226 & 49.83 & 117647 & 47.81 \\
\hline
\end{tabular}

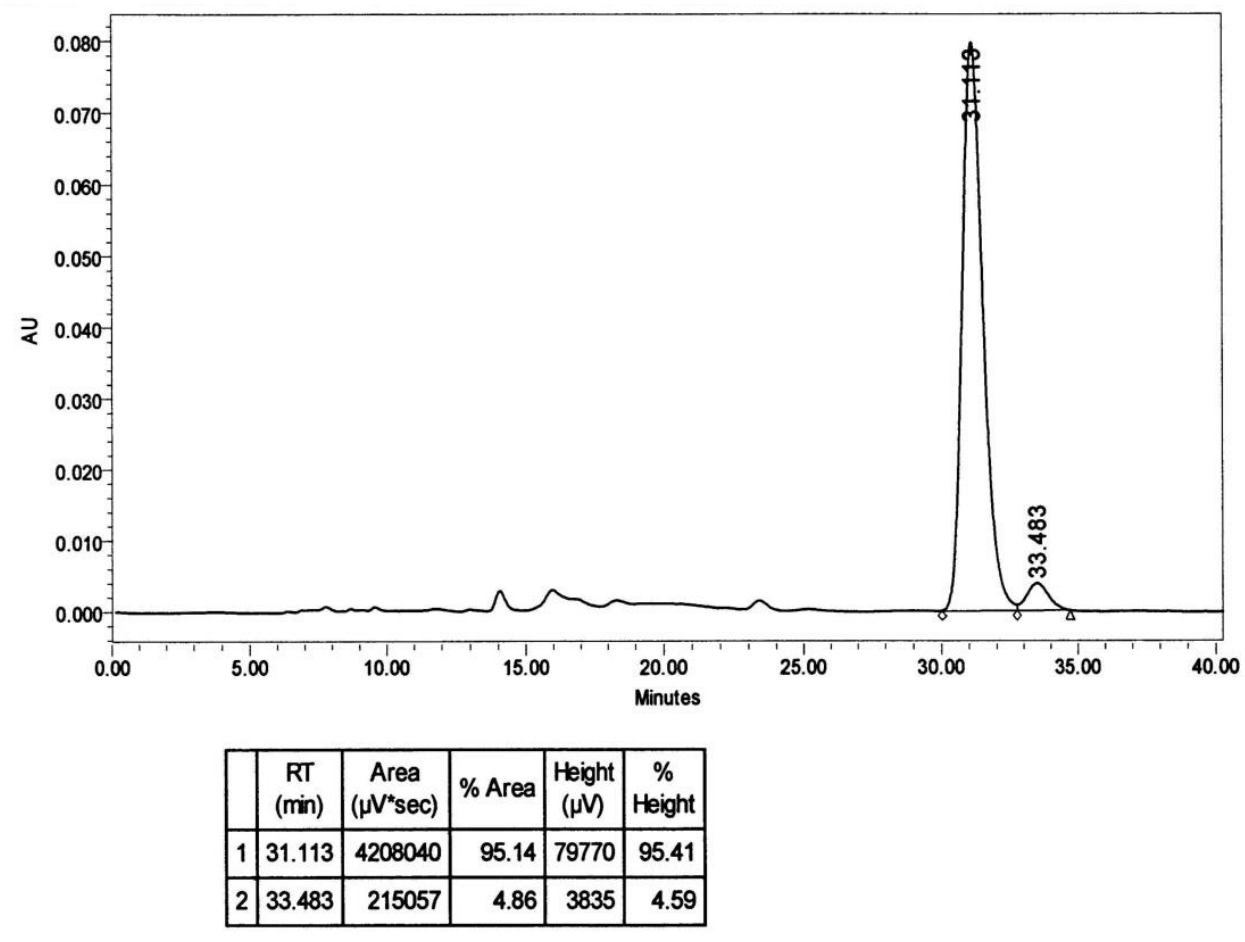



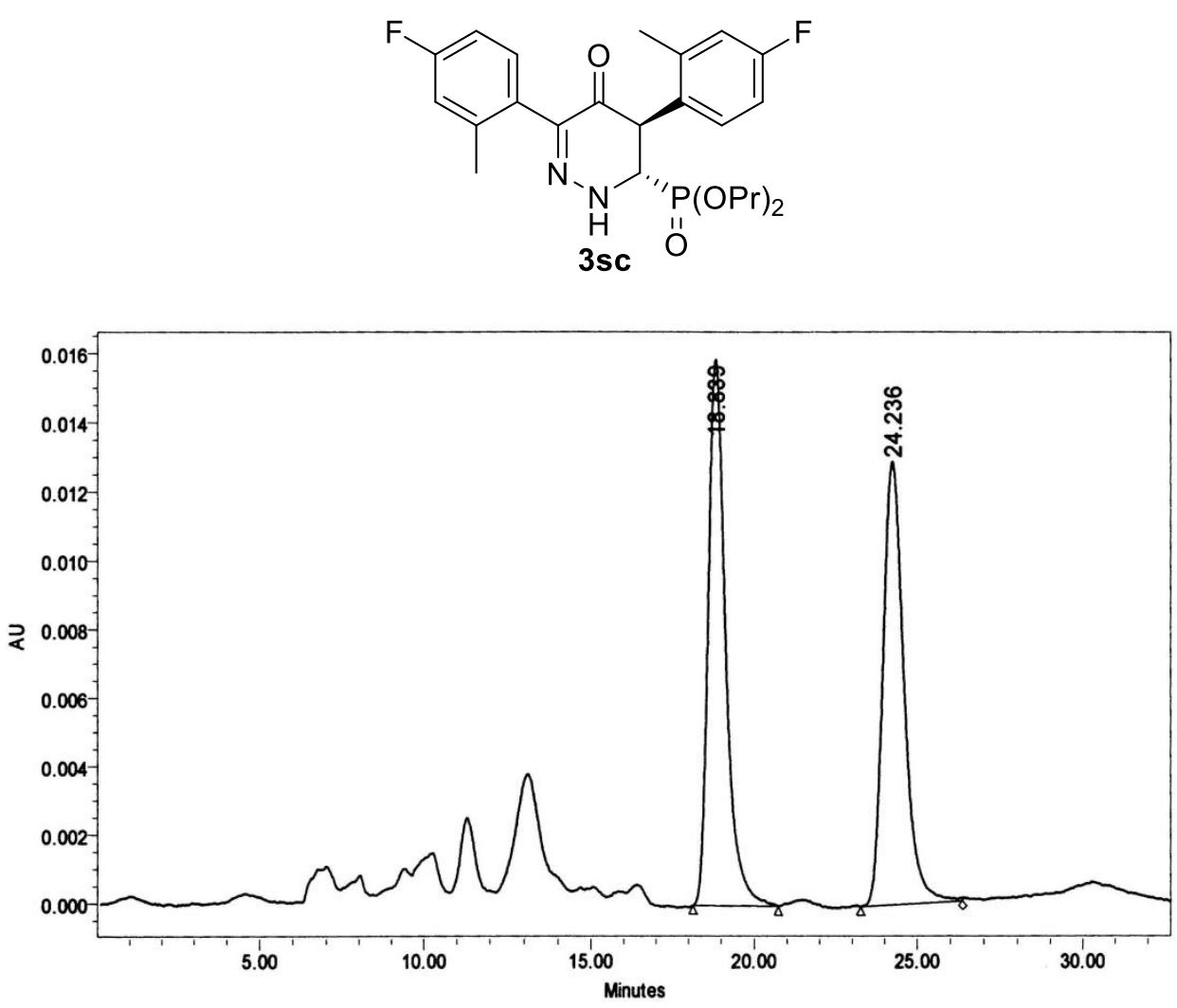

\begin{tabular}{|c|c|c|c|c|c|}
\hline & $\begin{array}{c}\mathrm{RT} \\
(\mathrm{min})\end{array}$ & $\begin{array}{c}\text { Area } \\
\left(\mu \mathrm{V}^{*} \mathrm{sec}\right)\end{array}$ & $\%$ Area & $\begin{array}{c}\text { Height } \\
(\mu \mathrm{V})\end{array}$ & $\begin{array}{c}\% \\
\text { Height }\end{array}$ \\
\hline 1 & 18.839 & 586024 & 50.40 & 15908 & 55.20 \\
\hline 2 & 24.236 & 576801 & 49.60 & 12912 & 44.80 \\
\hline
\end{tabular}

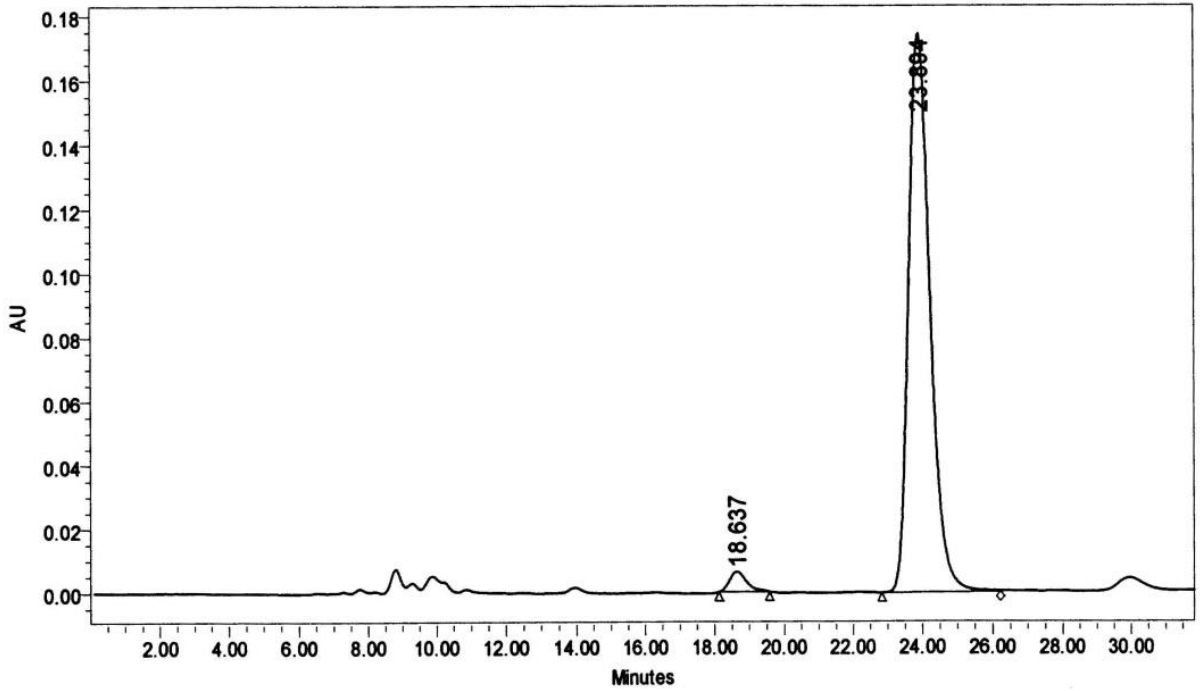

\begin{tabular}{|c|c|r|r|r|r|}
\hline & $\begin{array}{c}\mathrm{RT} \\
(\mathrm{min})\end{array}$ & $\begin{array}{c}\text { Area } \\
(\mu \mathrm{V} \text { sec })\end{array}$ & $\%$ Area & $\begin{array}{c}\text { Height } \\
(\mu \mathrm{V})\end{array}$ & $\begin{array}{c}\% \\
\text { Height }\end{array}$ \\
\hline 1 & 18.637 & 212606 & 2.76 & 6287 & 3.47 \\
\hline 2 & 23.894 & 7482286 & 97.24 & 174671 & 96.53 \\
\hline
\end{tabular}



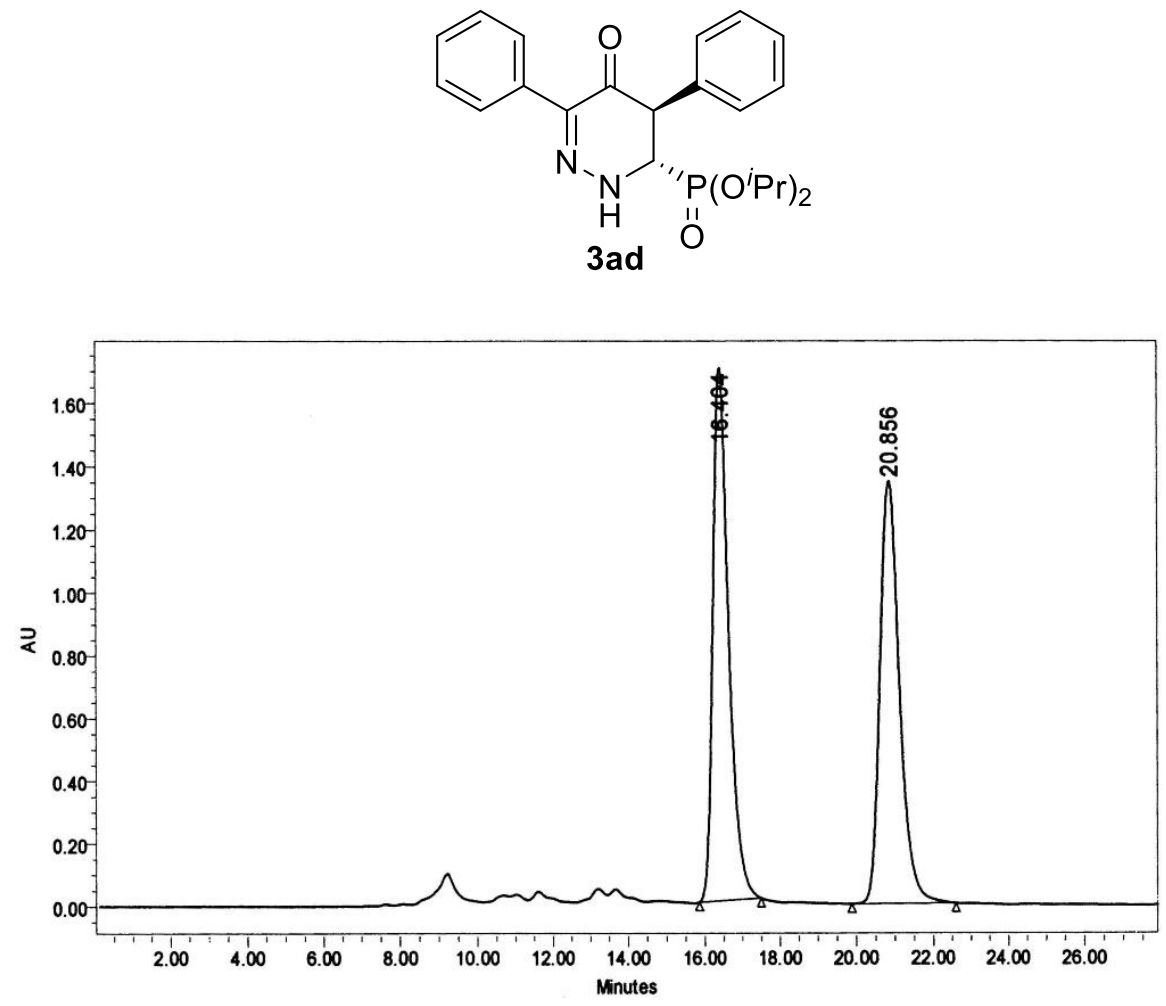

\begin{tabular}{|c|c|c|c|c|c|}
\hline & $\begin{array}{c}\text { RT } \\
(\mathrm{min})\end{array}$ & $\begin{array}{c}\text { Area } \\
(\mu \mathrm{V} * \mathrm{sec})\end{array}$ & $\%$ Area & $\begin{array}{c}\text { Height } \\
(\mu \mathrm{V})\end{array}$ & $\begin{array}{c}\% \\
\text { Height }\end{array}$ \\
\hline 1 & 16.404 & 49425417 & 51.07 & 1694584 & 55.74 \\
\hline 2 & 20.856 & 47350628 & 48.93 & 1345714 & 44.26 \\
\hline
\end{tabular}

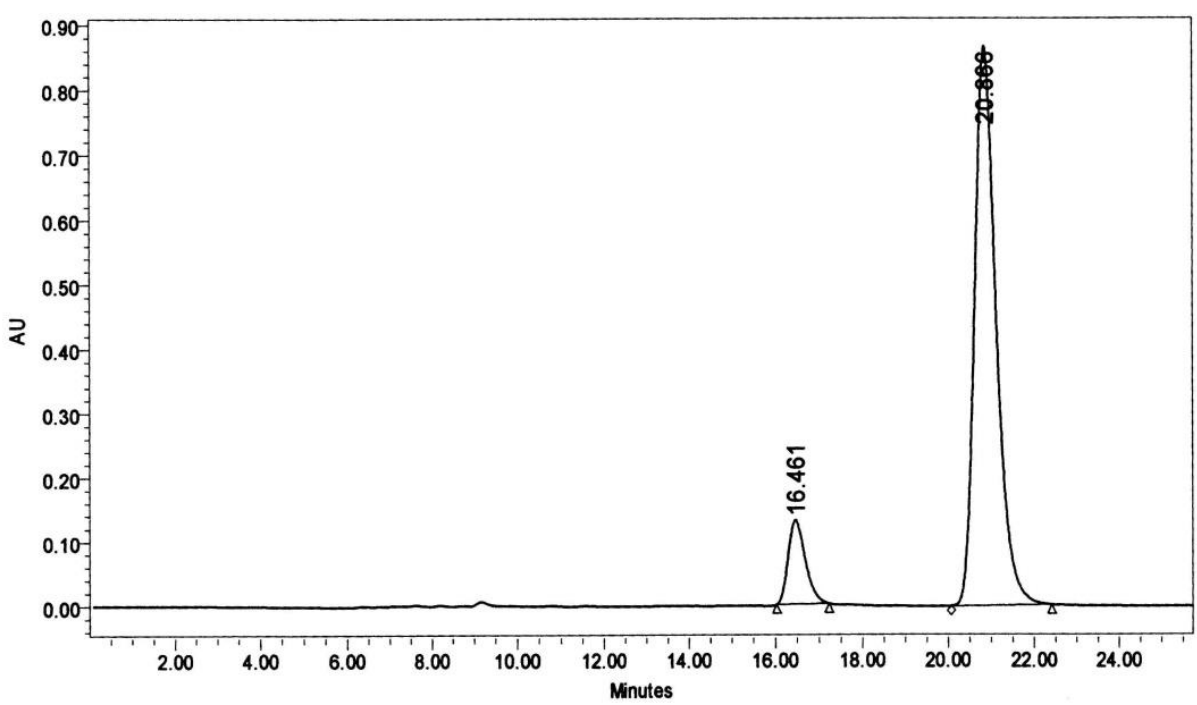

\begin{tabular}{|c|c|c|c|c|c|}
\hline & $\begin{array}{c}\mathrm{RT} \\
(\mathrm{min})\end{array}$ & $\begin{array}{c}\text { Area } \\
\left(\mu \mathrm{V}^{*} \mathrm{sec}\right)\end{array}$ & $\%$ Area & $\begin{array}{c}\text { Height } \\
(\mu \mathrm{V})\end{array}$ & $\begin{array}{c}\% \\
\text { Height }\end{array}$ \\
\hline 1 & 16.461 & 3564545 & 10.58 & 130860 & 13.12 \\
\hline 2 & 20.866 & 30126112 & 89.42 & 866693 & 86.88 \\
\hline
\end{tabular}



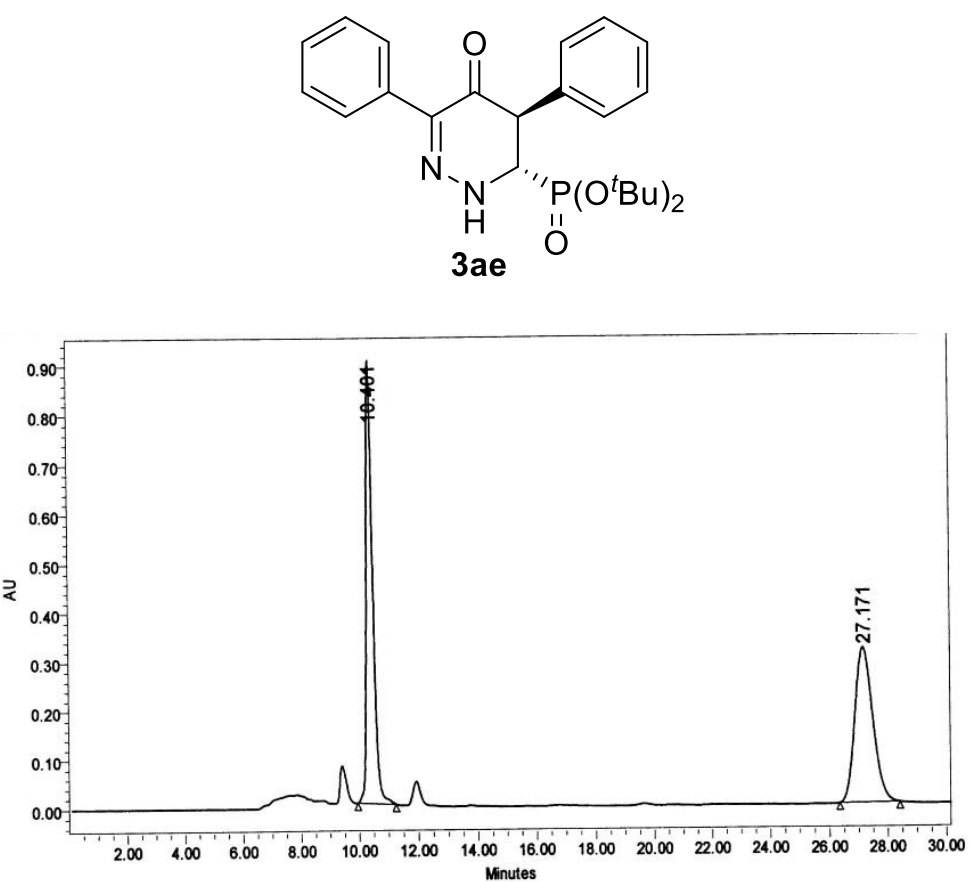

\begin{tabular}{|c|c|c|c|c|c|}
\hline & $\begin{array}{c}\mathrm{RT} \\
(\mathrm{min})\end{array}$ & $\begin{array}{c}\text { Area } \\
\left(\mu \mathrm{V}^{*} \mathrm{sec}\right)\end{array}$ & $\%$ Area & $\begin{array}{c}\text { Height } \\
(\mu \mathrm{V})\end{array}$ & $\begin{array}{c}\% \\
\text { Height }\end{array}$ \\
\hline 1 & 10.401 & 13961469 & 50.57 & 899899 & 73.98 \\
\hline 2 & 27.171 & 13646262 & 49.43 & 316544 & 26.02 \\
\hline
\end{tabular}

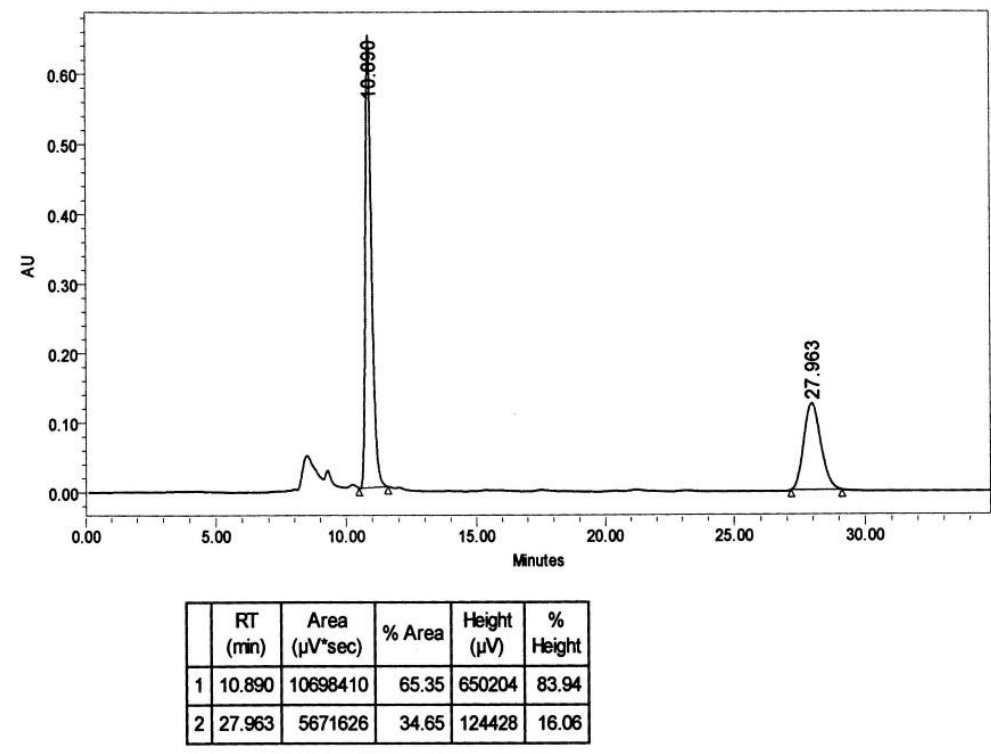



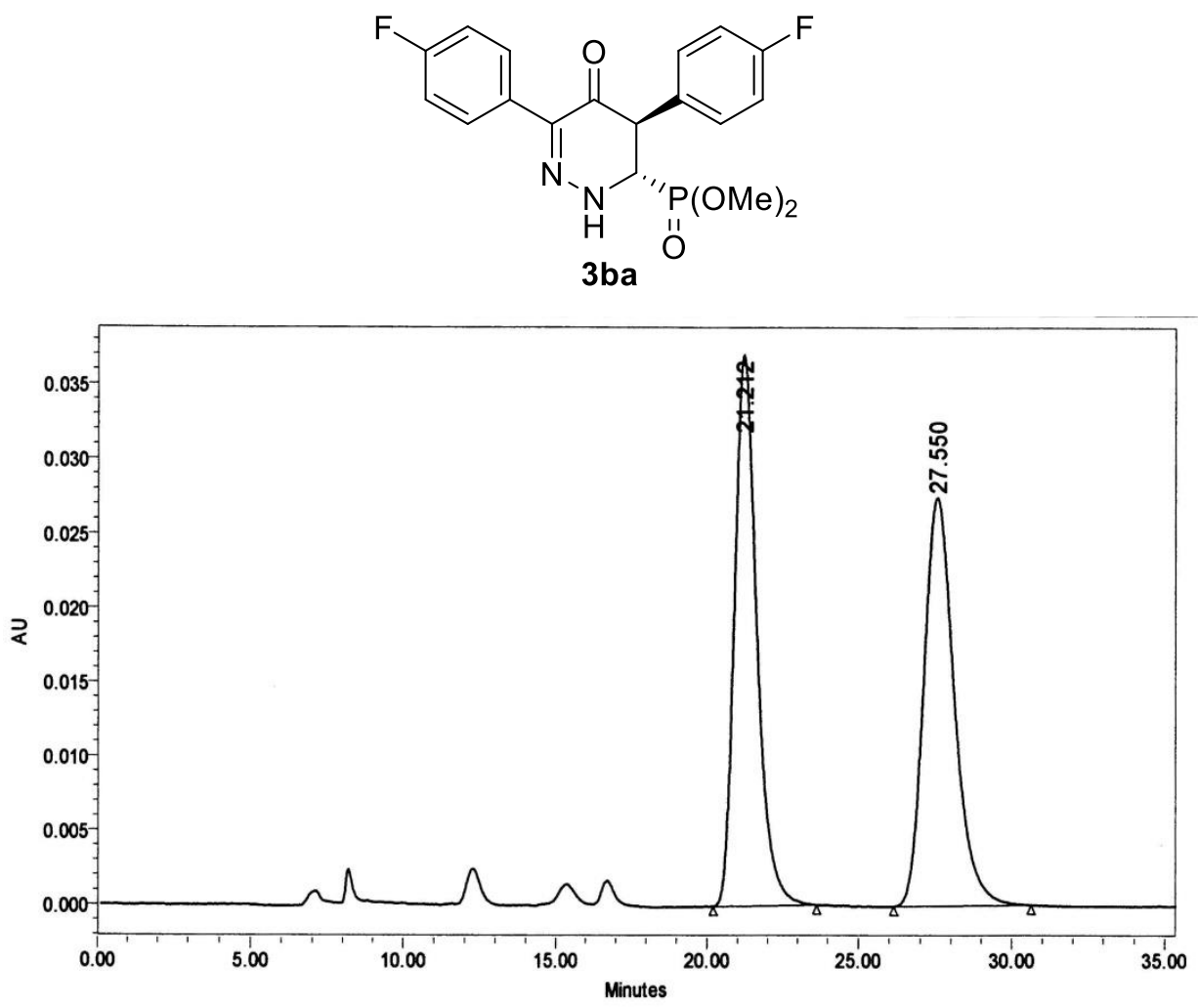

\begin{tabular}{|c|c|c|c|c|c|}
\hline & $\begin{array}{c}\mathrm{RT} \\
(\mathrm{min})\end{array}$ & $\begin{array}{c}\text { Area } \\
(\mu \mathrm{V} \text { sec })\end{array}$ & $\%$ Area & $\begin{array}{c}\text { Height } \\
(\mu \mathrm{V})\end{array}$ & $\begin{array}{c}\% \\
\text { Height }\end{array}$ \\
\hline 1 & 21.212 & 1909891 & 49.96 & 37061 & 57.37 \\
\hline 2 & 27.550 & 1913197 & 50.04 & 27535 & 42.63 \\
\hline
\end{tabular}

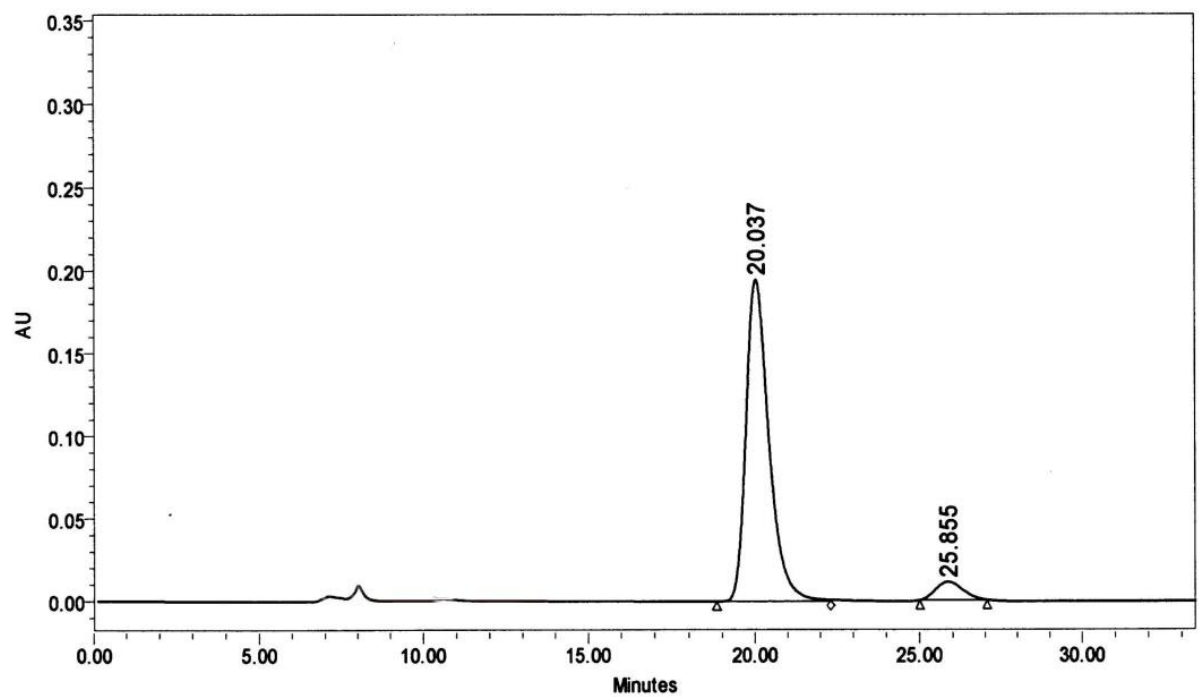

\begin{tabular}{|c|c|c|c|c|c|}
\hline & $\begin{array}{c}\mathrm{RT} \\
(\mathrm{min})\end{array}$ & $\begin{array}{c}\text { Area } \\
(\mu \mathrm{V} * \mathrm{sec})\end{array}$ & $\%$ Area & $\begin{array}{c}\text { Height } \\
(\mu \mathrm{V})\end{array}$ & $\begin{array}{c}\% \\
\text { Height }\end{array}$ \\
\hline 1 & 20.037 & 9516494 & 93.84 & 194715 & 94.66 \\
\hline 2 & 25.855 & 625110 & 6.16 & 10980 & 5.34 \\
\hline
\end{tabular}


<smiles>CCOC(=O)[C@@H]1NN=C(c2ccc(F)cc2)C(=O)C1c1ccc(F)cc1</smiles>

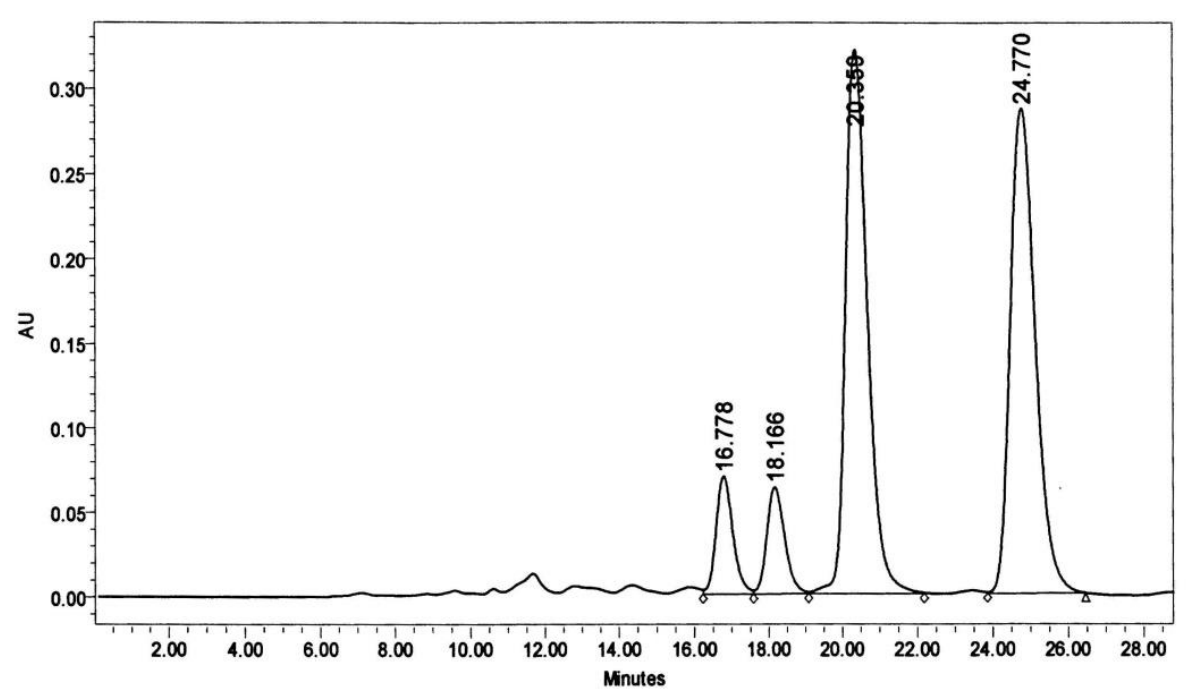

\begin{tabular}{|c|c|c|r|r|r|}
\hline & $\begin{array}{c}\mathrm{RT} \\
(\mathrm{min})\end{array}$ & $\begin{array}{c}\text { Area } \\
(\mu \mathrm{V} \text { 'sec })\end{array}$ & $\%$ Area & $\begin{array}{c}\text { Height } \\
(\mu \mathrm{V})\end{array}$ & $\begin{array}{c}\% \\
\text { Height }\end{array}$ \\
\hline 1 & 16.778 & 2215508 & 7.19 & 69860 & 9.43 \\
\hline 2 & 18.166 & 2146899 & 6.97 & 63302 & 8.55 \\
\hline 3 & 20.359 & 13178797 & 42.76 & 321093 & 43.36 \\
\hline 4 & 24.770 & 13281042 & 43.09 & 286298 & 38.66 \\
\hline
\end{tabular}

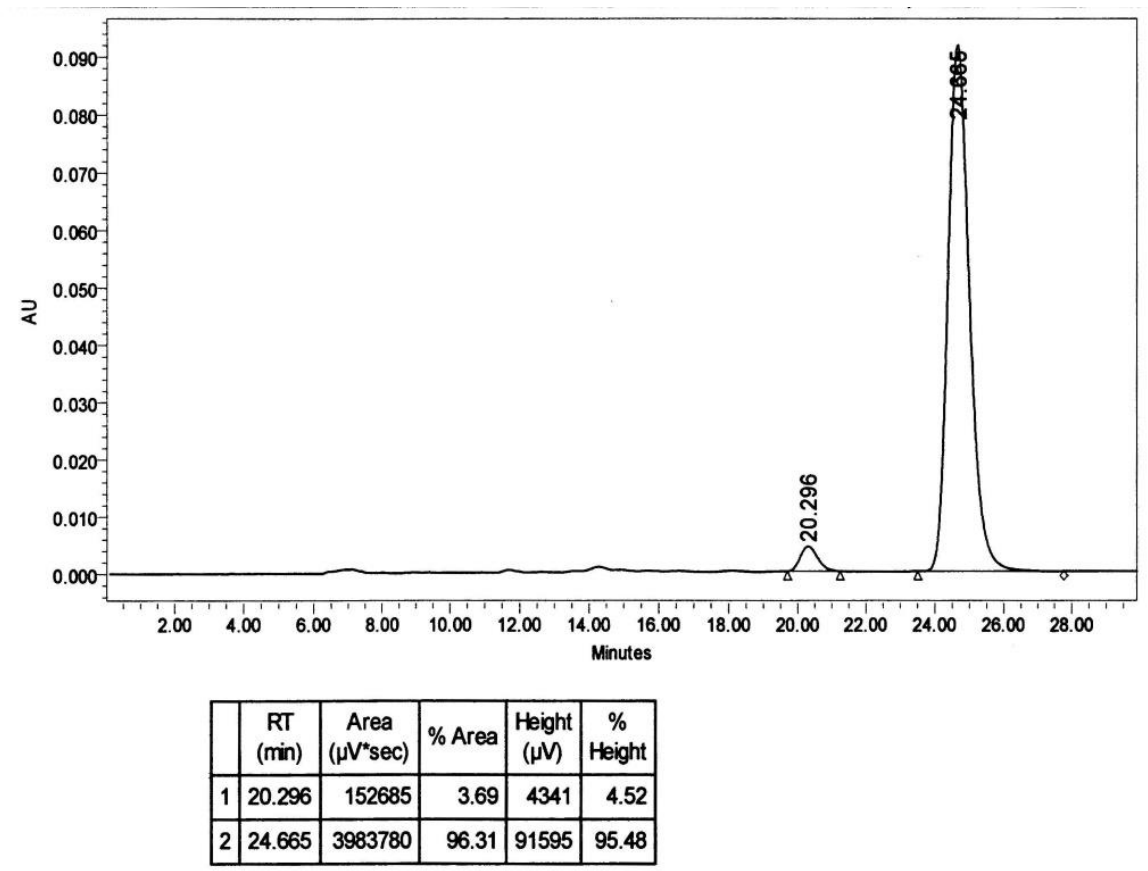



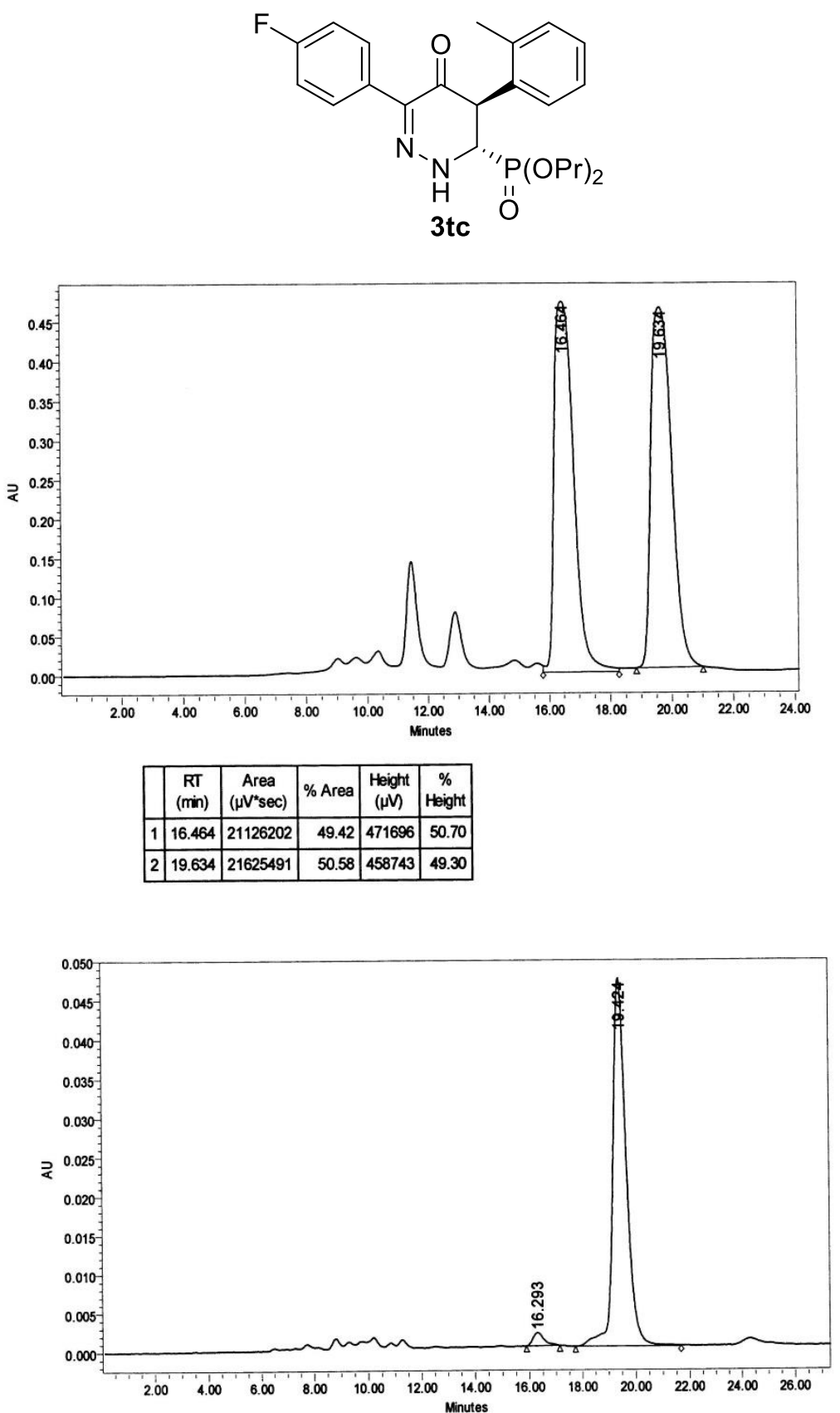

\begin{tabular}{|c|c|c|c|c|c|}
\hline & $\begin{array}{c}\mathrm{RT} \\
(\mathrm{min})\end{array}$ & $\begin{array}{c}\text { Area } \\
(\mu \mathrm{V} \text { 'sec })\end{array}$ & $\%$ Area & $\begin{array}{l}\text { Height } \\
(\mu \mathrm{V})\end{array}$ & $\begin{array}{c}\% \\
\text { Height }\end{array}$ \\
\hline 1 & 16.293 & 48864 & 2.88 & 1680 & 3.45 \\
\hline 2 & 19.424 & 1645068 & 97.12 & 47079 & 96.55 \\
\hline
\end{tabular}



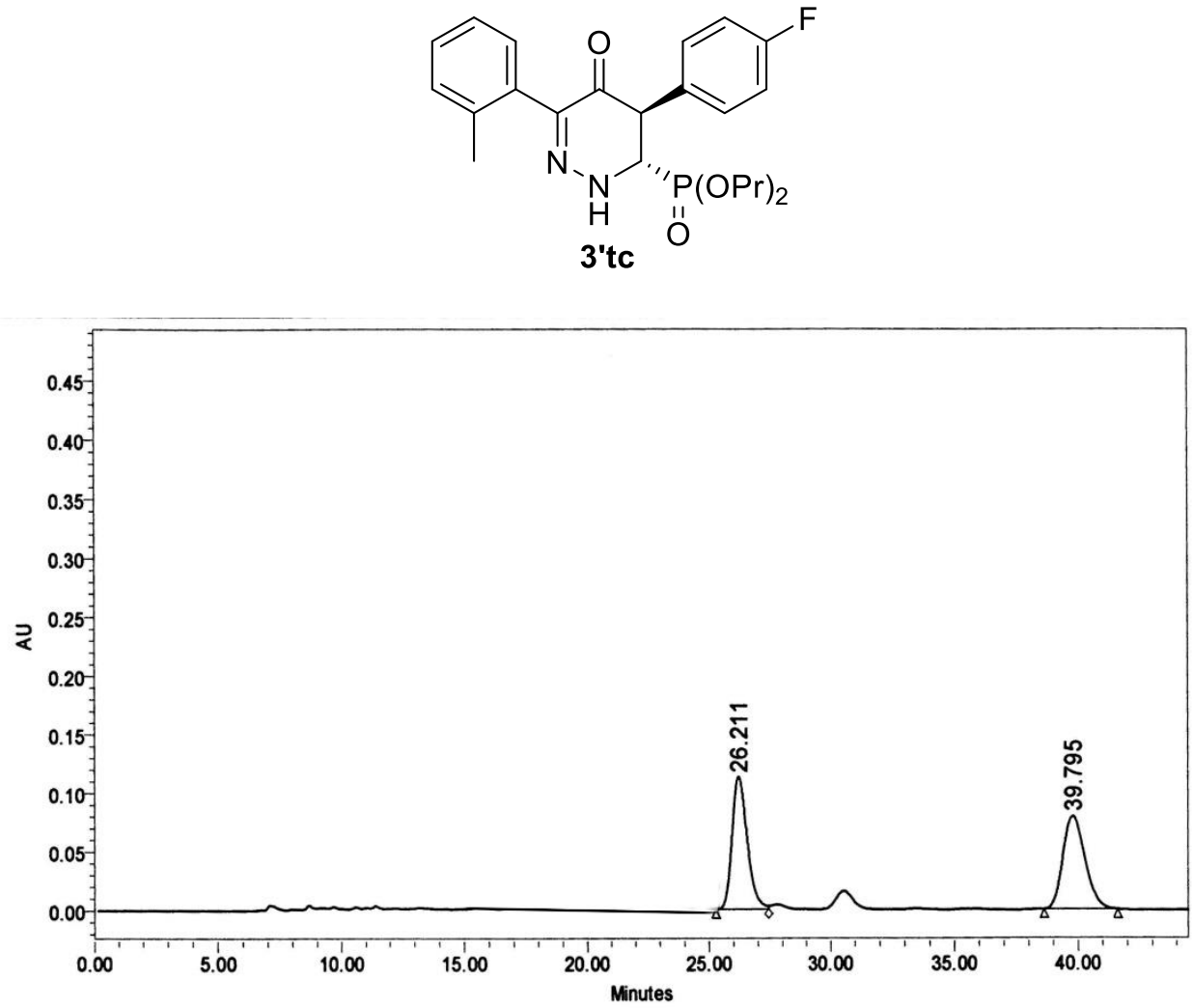

\begin{tabular}{|c|c|c|c|c|c|}
\hline & $\begin{array}{c}\mathrm{RT} \\
(\mathrm{min})\end{array}$ & $\begin{array}{c}\text { Area } \\
(\mu \mathrm{V} * \mathrm{sec})\end{array}$ & $\%$ Area & $\begin{array}{c}\text { Height } \\
(\mu \mathrm{V})\end{array}$ & $\begin{array}{c}\% \\
\text { Height }\end{array}$ \\
\hline 1 & 26.211 & 4938472 & 50.10 & 112906 & 58.75 \\
\hline 2 & 39.795 & 4918730 & 49.90 & 79275 & 41.25 \\
\hline
\end{tabular}

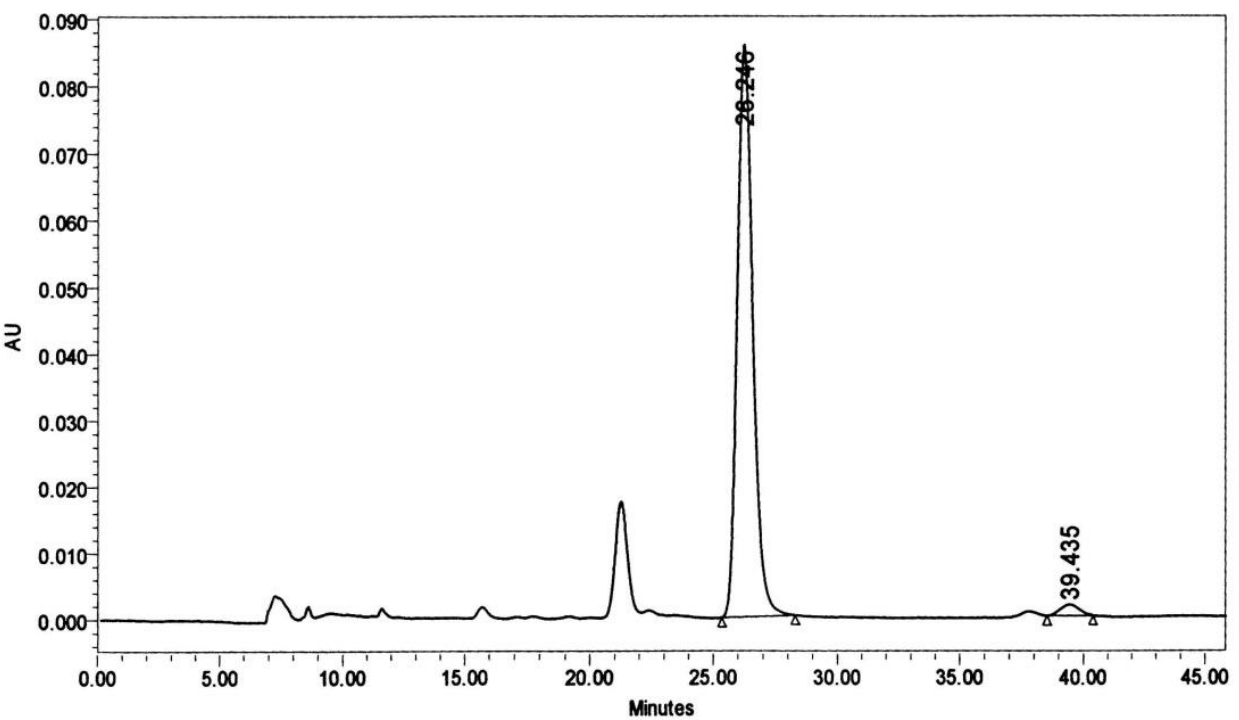

\begin{tabular}{|c|c|c|c|c|c|}
\hline & $\begin{array}{c}\text { RT } \\
(\mathrm{min})\end{array}$ & $\begin{array}{c}\text { Area } \\
\left(\mu \mathrm{V}^{*} \mathrm{sec}\right)\end{array}$ & $\%$ Area & $\begin{array}{c}\text { Height } \\
(\mu \mathrm{V})\end{array}$ & $\begin{array}{c}\% \\
\text { Height }\end{array}$ \\
\hline 1 & 26.246 & 3767011 & 97.72 & 85748 & 98.10 \\
\hline 2 & 39.435 & 87916 & 2.28 & 1664 & 1.90 \\
\hline
\end{tabular}



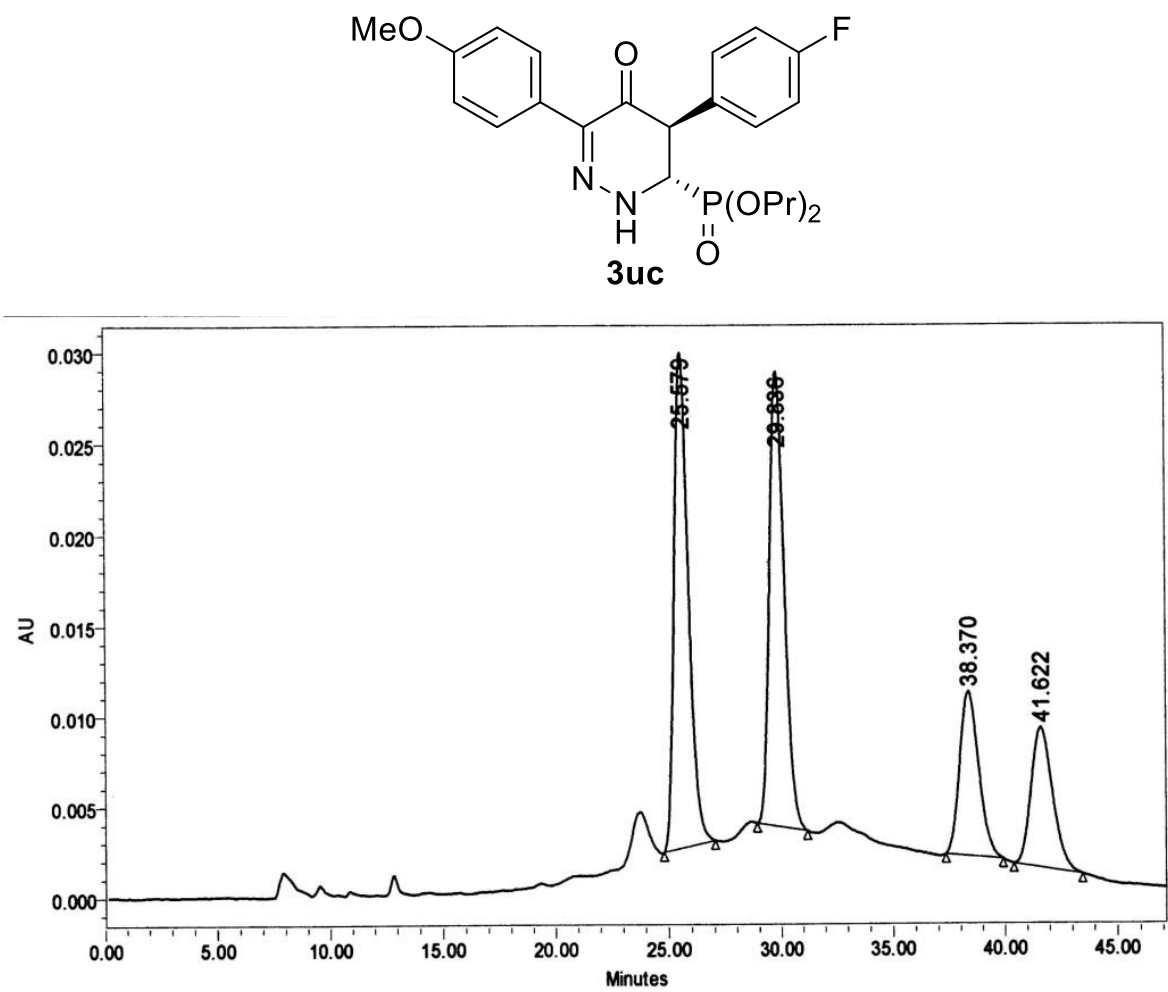

\begin{tabular}{|c|c|c|c|c|c|}
\hline & $\begin{array}{c}\mathrm{RT} \\
(\mathrm{min})\end{array}$ & $\begin{array}{c}\text { Area } \\
(\mu \mathrm{V} \text { *sec })\end{array}$ & $\%$ Area & $\begin{array}{c}\text { Height } \\
(\mu \mathrm{V})\end{array}$ & $\begin{array}{c}\% \\
\text { Height }\end{array}$ \\
\hline 1 & 25.579 & 1173694 & 34.49 & 27279 & 39.44 \\
\hline 2 & 29.836 & 1167225 & 34.30 & 25041 & 36.21 \\
\hline 3 & 38.370 & 543313 & 15.97 & 9095 & 13.15 \\
\hline 4 & 41.622 & 518368 & 15.23 & 7746 & 11.20 \\
\hline
\end{tabular}

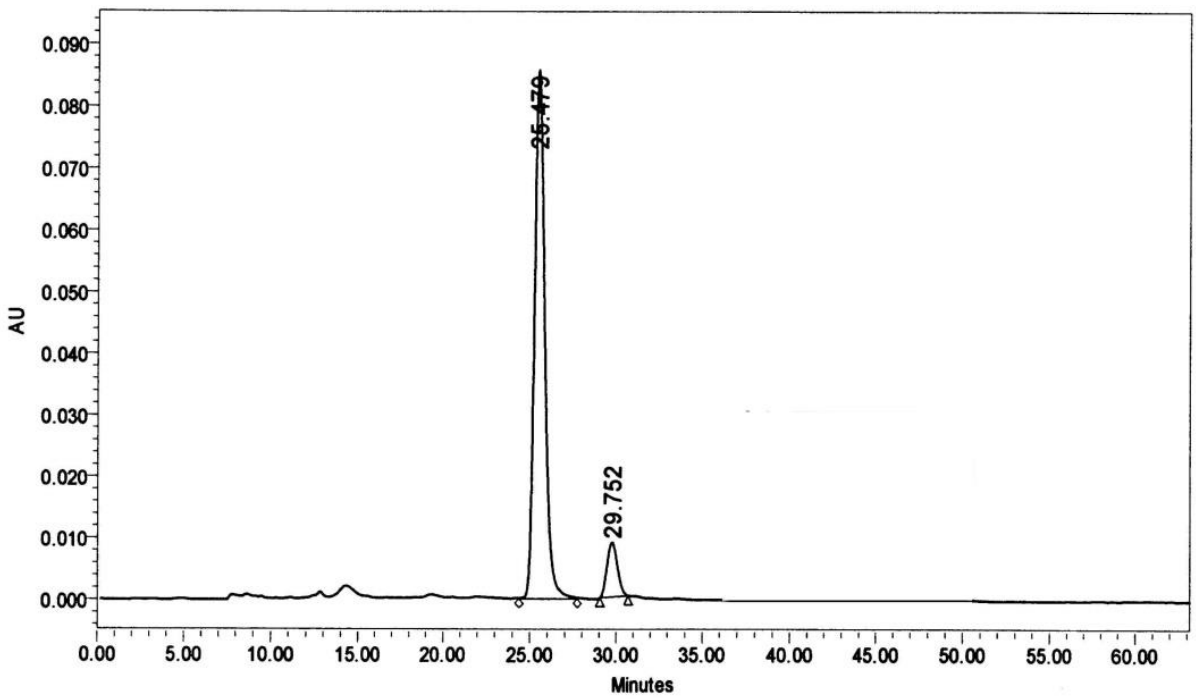

\begin{tabular}{|c|c|c|c|c|c|}
\hline & $\begin{array}{c}\mathrm{RT} \\
(\min )\end{array}$ & $\begin{array}{c}\text { Area } \\
(\mu \mathrm{V} * \mathrm{sec})\end{array}$ & $\%$ Area & $\begin{array}{c}\text { Height } \\
(\mu \mathrm{V})\end{array}$ & $\begin{array}{c}\% \\
\text { Height }\end{array}$ \\
\hline 1 & 25.479 & 3521755 & 90.45 & 85697 & 90.68 \\
\hline 2 & 29.752 & 371877 & 9.55 & 8813 & 9.32 \\
\hline
\end{tabular}


<smiles>COC(=O)OC(=O)[C@H]1NN=C(c2ccc(F)cc2)C(=O)C1c1ccc(OC)cc1</smiles>

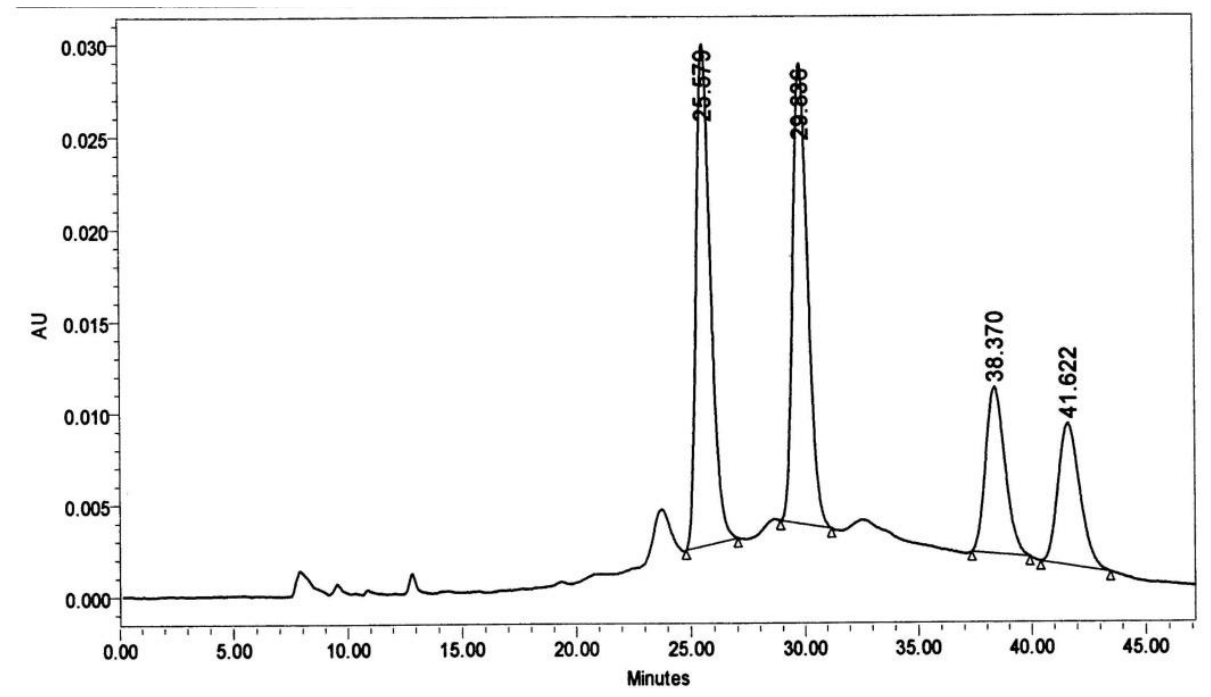

\begin{tabular}{|c|c|c|c|c|c|}
\hline & $\begin{array}{c}\mathrm{RT} \\
(\mathrm{min})\end{array}$ & $\begin{array}{c}\text { Area } \\
\left(\mu \mathrm{V}^{*} \mathrm{sec}\right)\end{array}$ & $\%$ Area & $\begin{array}{c}\text { Height } \\
(\mu \mathrm{V})\end{array}$ & $\begin{array}{c}\% \\
\text { Height }\end{array}$ \\
\hline 1 & 25.579 & 1173694 & 34.49 & 27279 & 39.44 \\
\hline 2 & 29.836 & 1167225 & 34.30 & 25041 & 36.21 \\
\hline 3 & 38.370 & 543313 & 15.97 & 9095 & 13.15 \\
\hline 4 & 41.622 & 518368 & 15.23 & 7746 & 11.20 \\
\hline
\end{tabular}

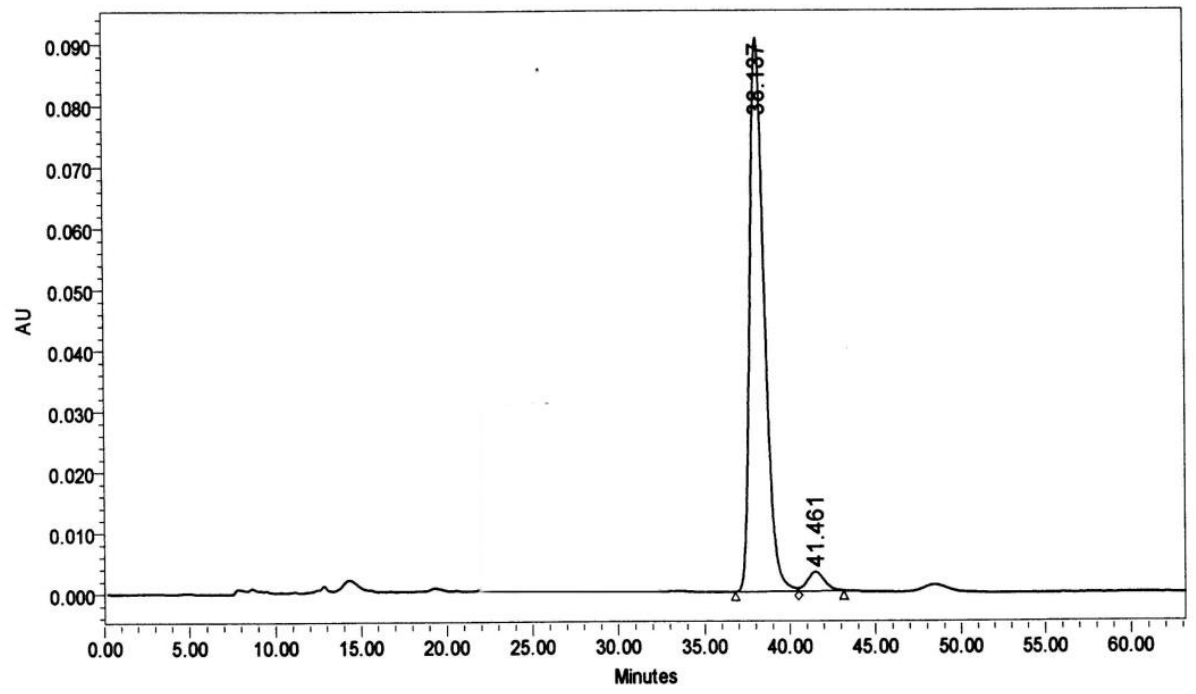

\begin{tabular}{|c|c|c|c|c|c|}
\hline & $\begin{array}{c}\mathrm{RT} \\
(\mathrm{min})\end{array}$ & $\begin{array}{c}\text { Area } \\
\left(\mu \mathrm{V}^{*} \mathrm{sec}\right)\end{array}$ & $\%$ Area & $\begin{array}{c}\text { Height } \\
(\mu \mathrm{V})\end{array}$ & $\begin{array}{c}\% \\
\text { Height }\end{array}$ \\
\hline 1 & 38.137 & 5391958 & 96.13 & 90688 & 96.65 \\
\hline 2 & 41.461 & 216981 & 3.87 & 3141 & 3.35 \\
\hline
\end{tabular}



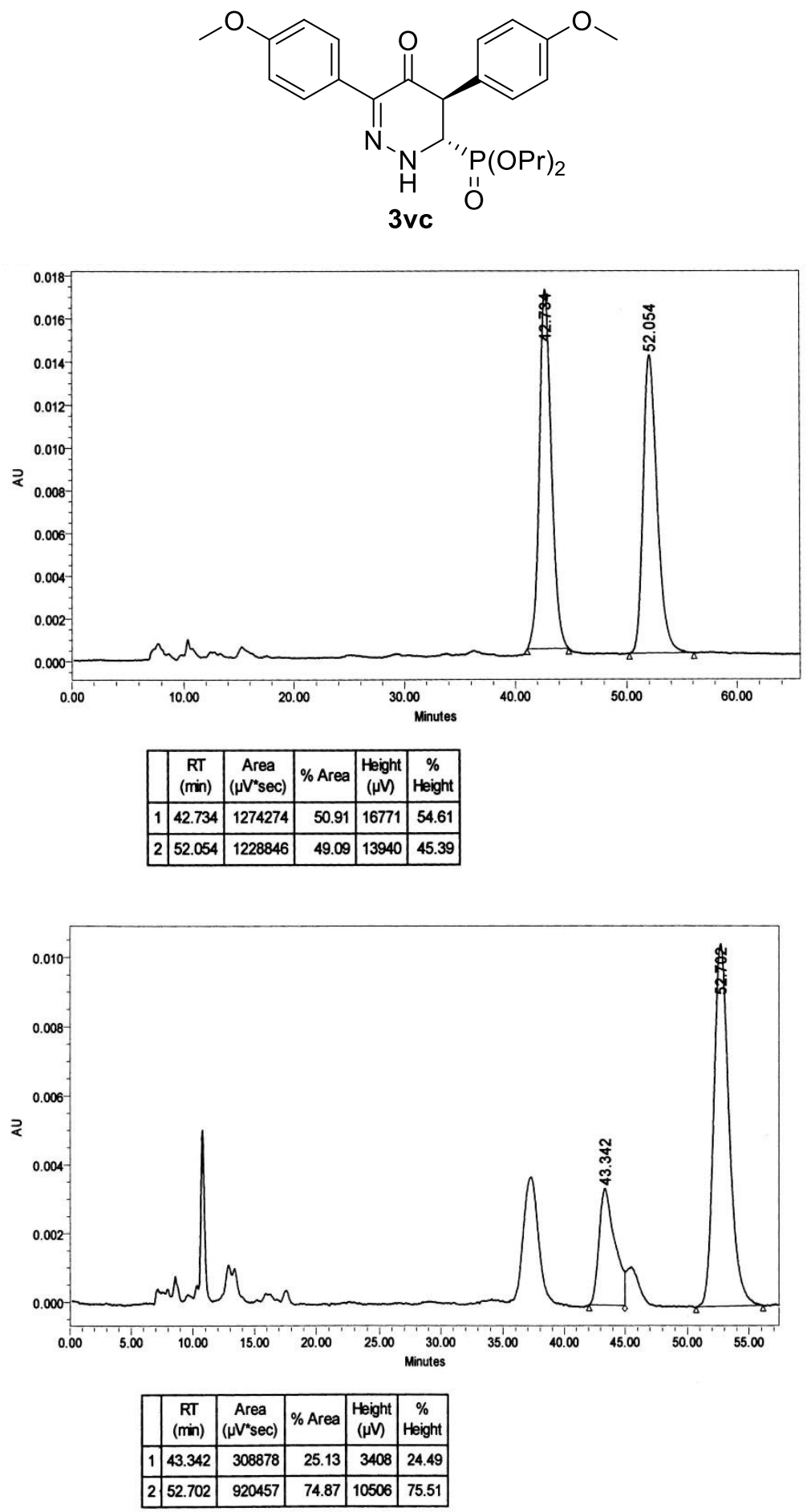


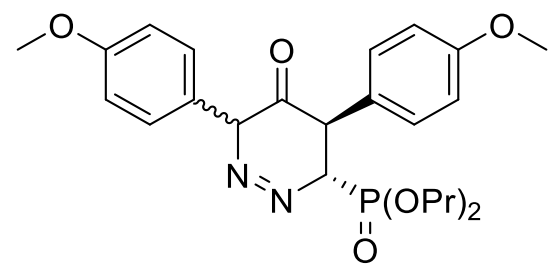

9vc

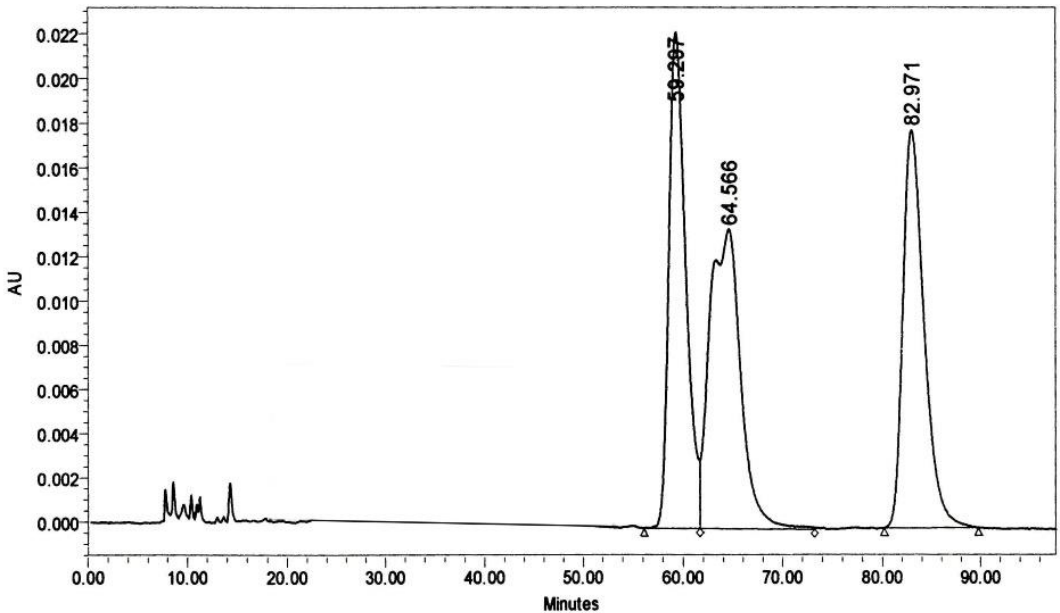

\begin{tabular}{|c|c|c|c|c|c|}
\hline & $\begin{array}{c}\mathrm{RT} \\
(\mathrm{min})\end{array}$ & $\begin{array}{c}\text { Area } \\
(\mu \mathrm{V} \text { sec })\end{array}$ & $\%$ Area & $\begin{array}{c}\text { Height } \\
(\mu \mathrm{V})\end{array}$ & $\begin{array}{c}\% \\
\text { Height }\end{array}$ \\
\hline 1 & 59.297 & 2592155 & 31.52 & 22369 & 41.50 \\
\hline 2 & 64.566 & 2947951 & 35.85 & 13567 & 25.17 \\
\hline 3 & 82.971 & 2683200 & 32.63 & 17965 & 33.33 \\
\hline
\end{tabular}

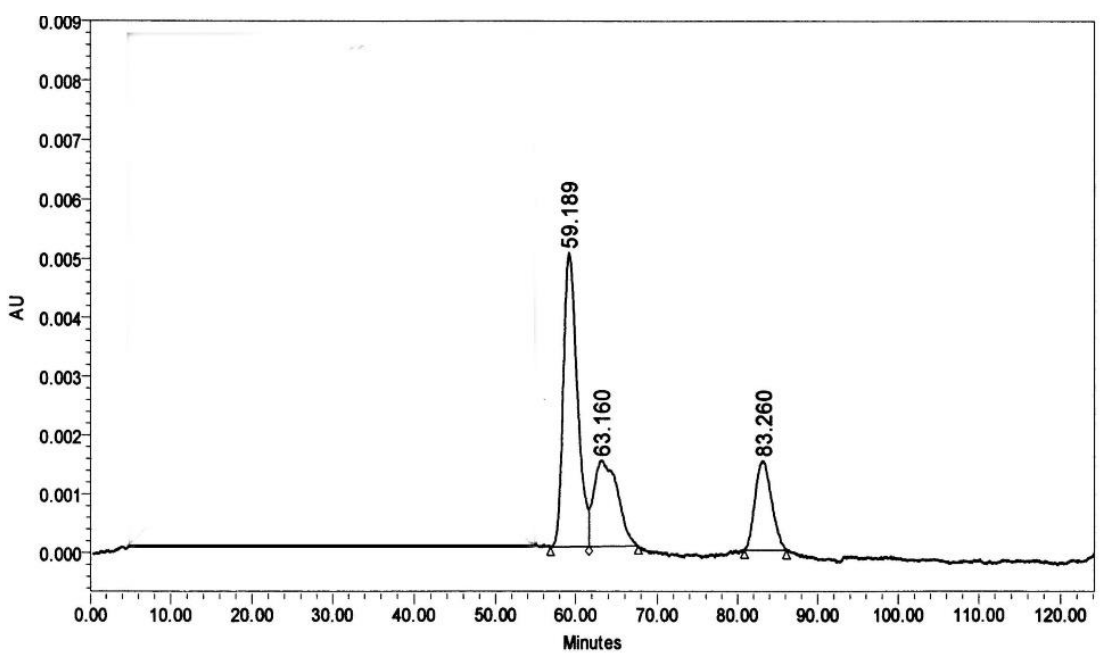

\begin{tabular}{|c|c|c|c|c|c|}
\hline & $\begin{array}{c}\mathrm{RT} \\
(\mathrm{min})\end{array}$ & $\begin{array}{c}\text { Area } \\
\left(\mu \mathrm{V}^{*} \mathrm{sec}\right)\end{array}$ & $\%$ Area & $\begin{array}{c}\text { Height } \\
(\mu \mathrm{N})\end{array}$ & $\begin{array}{c}\% \\
\text { Height }\end{array}$ \\
\hline 1 & 59.189 & 605170 & 54.11 & 4994 & 62.69 \\
\hline 2 & 63.160 & 295885 & 26.46 & 1459 & 18.32 \\
\hline 3 & 83.260 & 217372 & 19.44 & 1513 & 18.99 \\
\hline
\end{tabular}


<smiles>O=[PH](O)[PH2+]C1NN=C(c2ccc(F)cc2)[C@H](O)[C@H]1c1ccc(F)cc1</smiles>

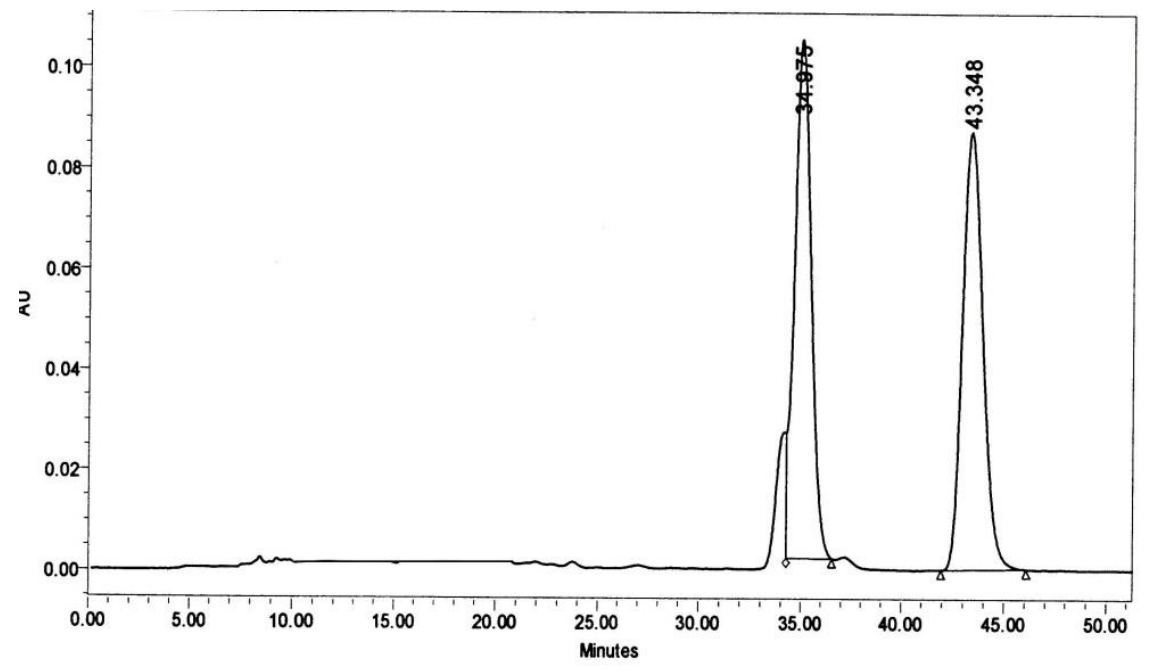

\begin{tabular}{|c|c|c|c|c|c|}
\hline & $\begin{array}{c}\text { RT } \\
(\mathrm{min})\end{array}$ & $\begin{array}{c}\text { Area } \\
(\mu \mathrm{V} \text { *sec })\end{array}$ & $\%$ Area & $\begin{array}{c}\text { Height } \\
(\mu \mathrm{V})\end{array}$ & $\begin{array}{c}\% \\
\text { Height }\end{array}$ \\
\hline 1 & 34.975 & 6021197 & 49.93 & 103131 & 54.17 \\
\hline 2 & 43.348 & 6037520 & 50.07 & 87245 & 45.83 \\
\hline
\end{tabular}

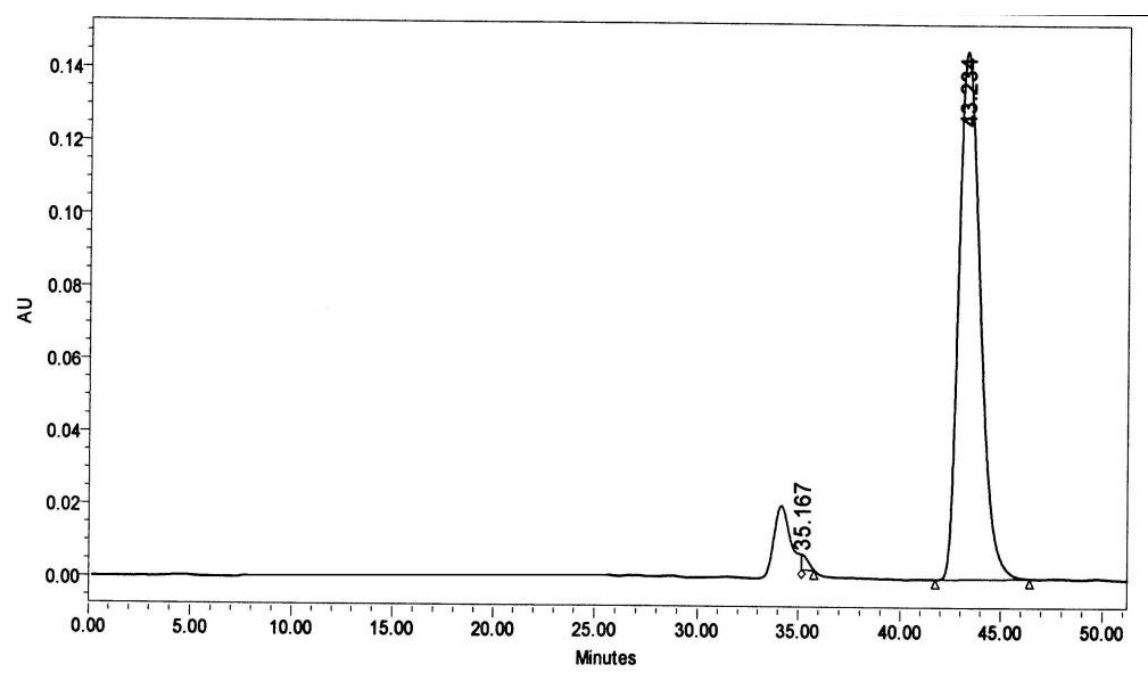

\begin{tabular}{|r|c|r|r|r|r|}
\hline & $\begin{array}{c}\mathrm{RT} \\
(\mathrm{min})\end{array}$ & $\begin{array}{c}\text { Area } \\
(\mu \mathrm{V} * \mathrm{sec})\end{array}$ & $\%$ Area & $\begin{array}{c}\text { Height } \\
(\mu \mathrm{V})\end{array}$ & $\begin{array}{c}\% \\
\text { Height }\end{array}$ \\
\hline 1 & 35.167 & 88160 & 0.80 & 4229 & 2.83 \\
\hline 2 & 43.234 & 10897642 & 99.20 & 145164 & 97.17 \\
\hline
\end{tabular}



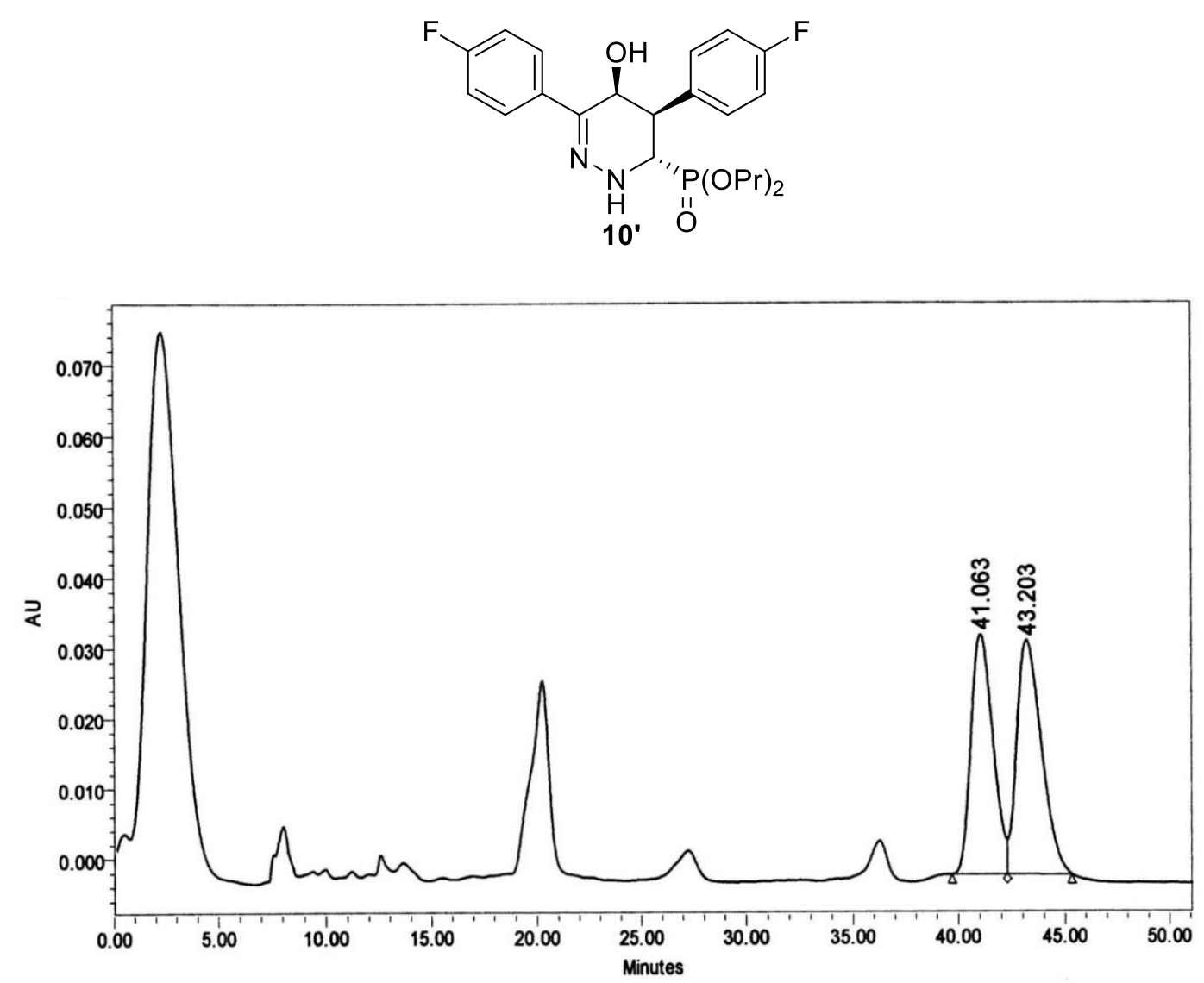

\begin{tabular}{|c|c|c|c|c|c|}
\hline & $\begin{array}{c}\mathrm{RT} \\
(\mathrm{min})\end{array}$ & $\begin{array}{c}\text { Area } \\
(\mu \mathrm{V} * \mathrm{sec})\end{array}$ & $\%$ Area & $\begin{array}{c}\text { Height } \\
(\mu \mathrm{V})\end{array}$ & $\begin{array}{c}\% \\
\text { Height }\end{array}$ \\
\hline 1 & 41.063 & 2436444 & 47.32 & 34218 & 50.60 \\
\hline 2 & 43.203 & 2712817 & 52.68 & 33408 & 49.40 \\
\hline
\end{tabular}

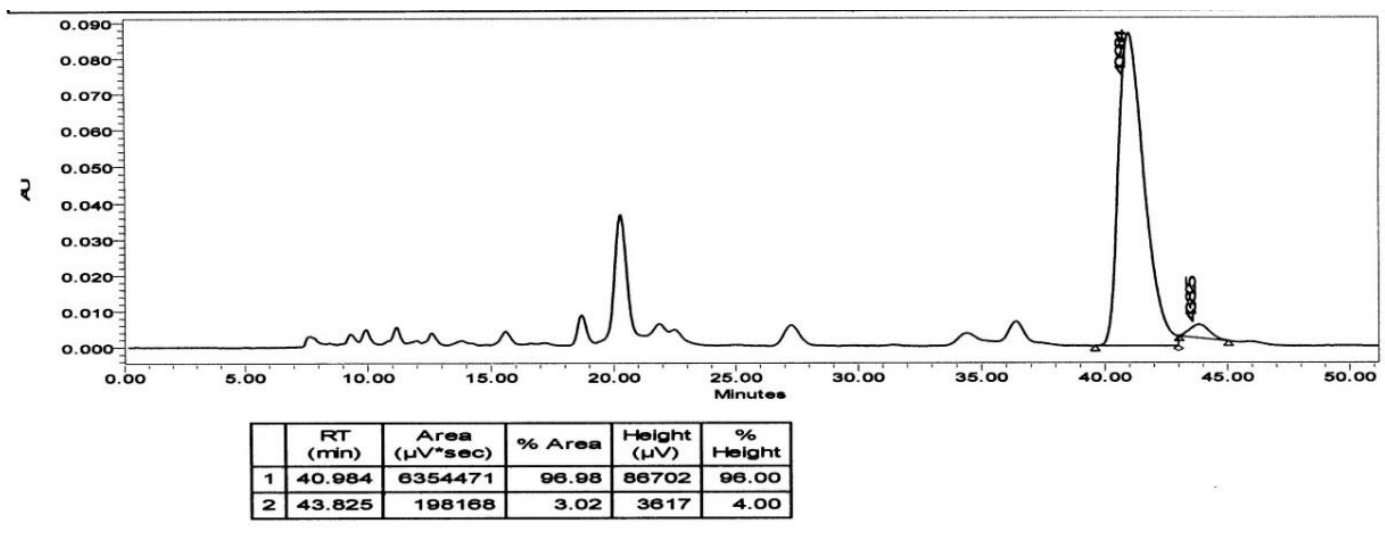



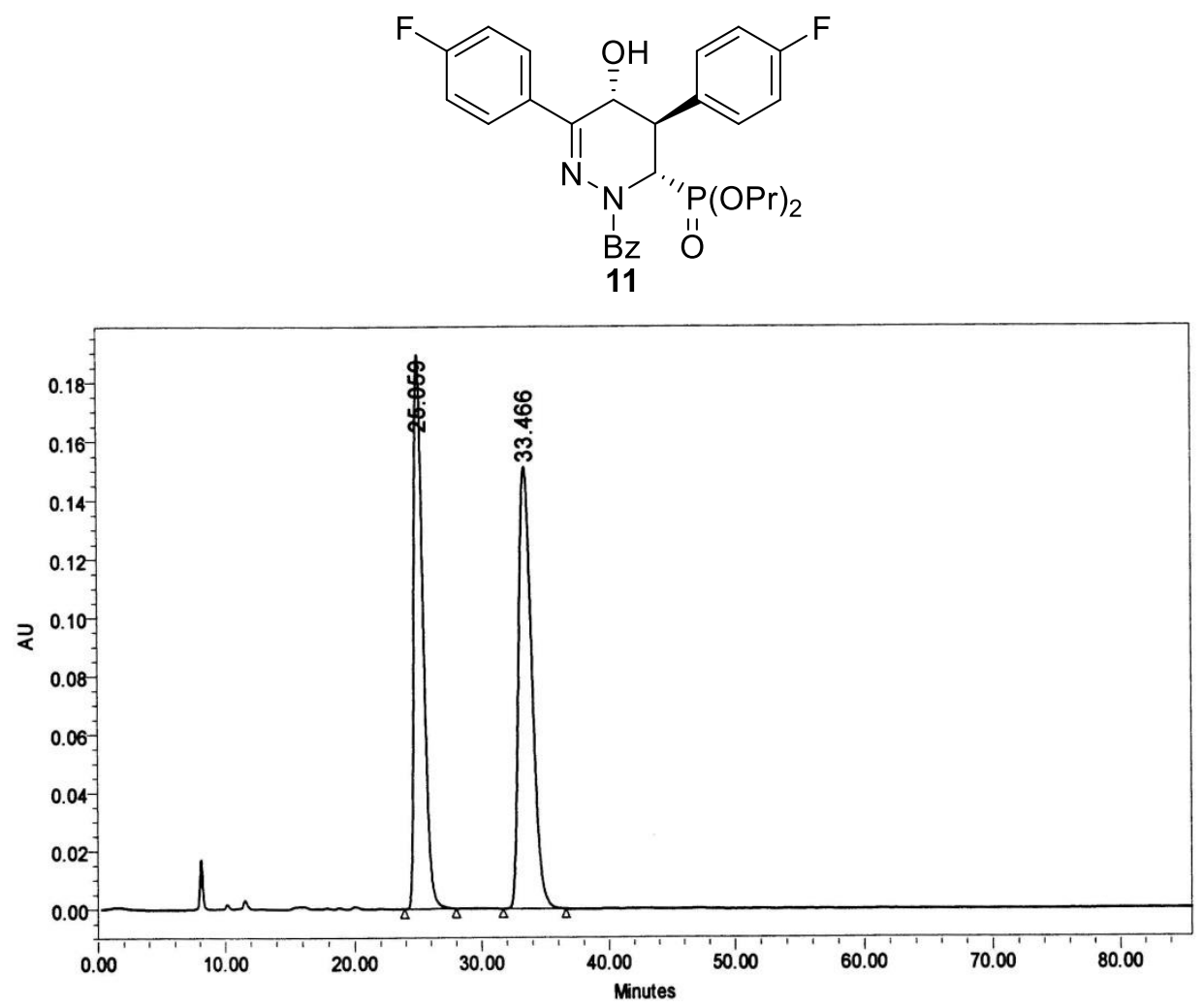

\begin{tabular}{|c|c|c|c|c|c|}
\hline & $\begin{array}{c}\mathrm{RT} \\
(\mathrm{min})\end{array}$ & $\begin{array}{c}\text { Area } \\
(\mu \mathrm{V} * \mathrm{sec})\end{array}$ & $\%$ Area & $\begin{array}{c}\text { Height } \\
(\mu \mathrm{V})\end{array}$ & $\begin{array}{c}\% \\
\text { Height }\end{array}$ \\
\hline 1 & 25.059 & 9771367 & 47.26 & 189500 & 55.62 \\
\hline 2 & 33.466 & 10905291 & 52.74 & 151219 & 44.38 \\
\hline
\end{tabular}

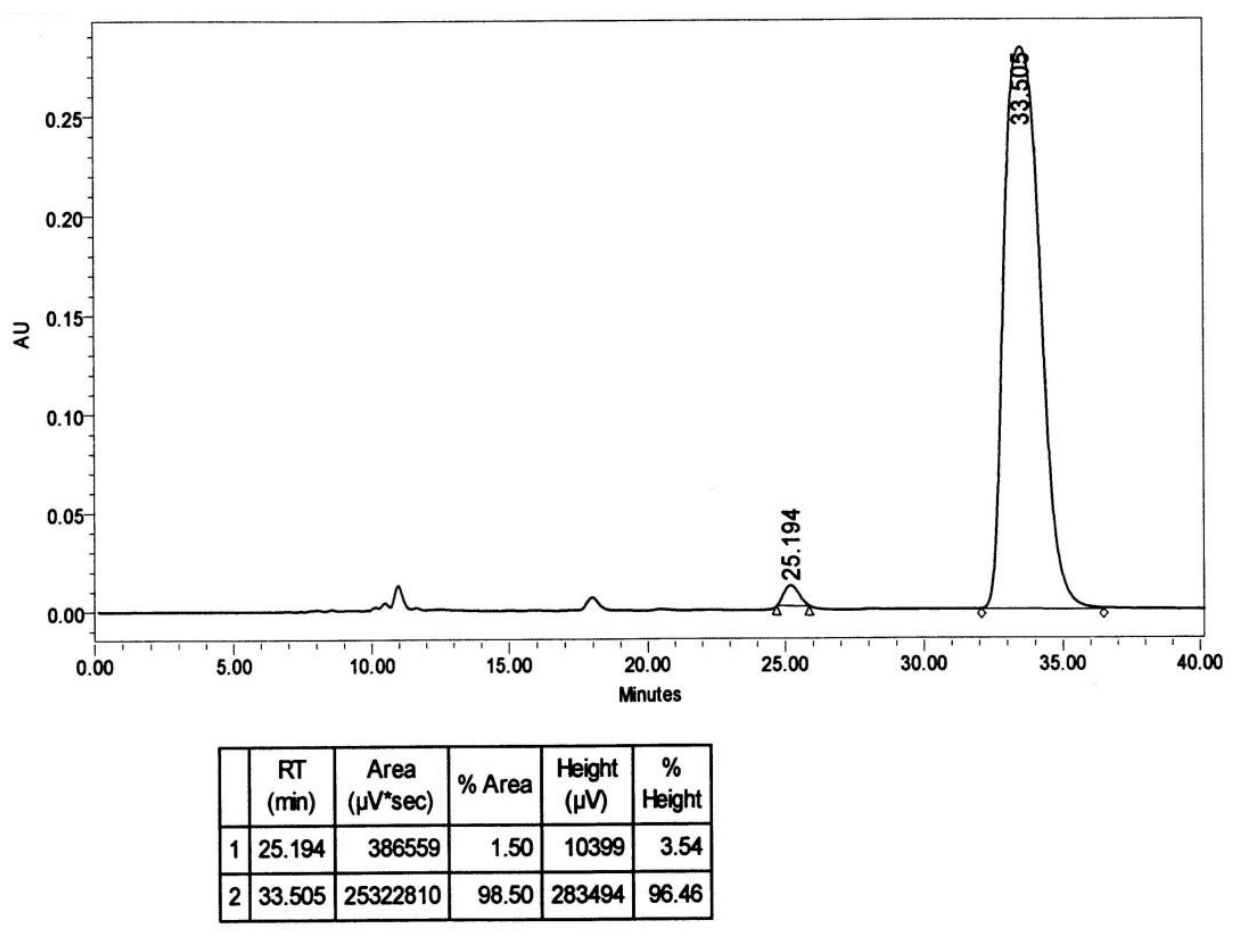

